\title{
The Distancing-Embracing model of the enjoyment of negative emotions in art reception
}

\author{
Winfried Menninghaus ${ }^{1}$ \\ Department of Language and Literature, Max Planck Institute for Empirical \\ Aesthetics, 60322 Frankfurt am Main, Germany \\ w.m@aesthetics.mpg.de \\ Valentin Wagner \\ Department of Language and Literature, Max Planck Institute for Empirical \\ Aesthetics, 60322 Frankfurt am Main, Germany \\ valentin.wagner@aesthetics.mpg.de \\ Julian Hanich \\ Department of Arts, Culture and Media, University of Groningen, 9700 AB \\ Groningen, The Netherlands \\ j.hanich@rug.nl

\section{Eugen Wassiliwizky} \\ Department of Language and Literature, Max Planck Institute for Empirical \\ Aesthetics, 60322 Frankfurt am Main, Germany \\ eugen.wassiliwizky@aesthetics.mpg.de \\ Thomas Jacobsen \\ Experimental Psychology Unit, Helmut Schmidt University/University of the \\ Federal Armed Forces Hamburg, 22043 Hamburg, Germany \\ jacobsen@hsu-hh.de

\section{Stefan Koelsch} \\ University of Bergen, 5020 Bergen, Norway \\ stefan.koelsch@uib.no
}

\begin{abstract}
Why are negative emotions so central in art reception far beyond tragedy? Revisiting classical aesthetics in the light of recent psychological research, we present a novel model to explain this much discussed (apparent) paradox. We argue that negative emotions are an important resource for the arts in general, rather than a special license for exceptional art forms only. The underlying rationale is that negative emotions have been shown to be particularly powerful in securing attention, intense emotional involvement, and high memorability, and hence is precisely what artworks strive for. Two groups of processing mechanisms are identified that conjointly adopt the particular powers of negative emotions for art's purposes. The first group consists of psychological distancing mechanisms that are activated along with the cognitive schemata of art, representation, and fiction. These schemata imply personal safety and control over continuing or discontinuing exposure to artworks, thereby preventing negative emotions from becoming outright incompatible with expectations of enjoyment. This distancing sets the stage for a second group of processing components that allow art recipients to positively embrace the experiencing of negative emotions, thereby rendering art reception more intense, more interesting, more emotionally moving, more profound, and occasionally even more beautiful. These components include compositional interplays of positive and negative emotions, the effects of aesthetic virtues of using the media of (re)presentation (musical sound, words/language, color, shapes) on emotion perception, and meaning-making efforts. Moreover, our DistancingEmbracing model proposes that concomitant mixed emotions often help integrate negative emotions into altogether pleasurable trajectories.
\end{abstract}

Keywords: aesthetic enjoyment; aesthetic virtues; art schema; genre effects; interplays of positive and negative emotions; meaning making; mixed emotions 
Winfried Menninghaus is the Director of the Department of Language and Literature at the Max Planck Institute for Empirical Aesthetics (Frankfurt am Main). He previously held professorships at Freie Universität Berlin, University of Jerusalem, University of California, Berkeley, Yale University, Princeton University, and Rice University. His fields of research are rhetoric and poetics, philosophical and empirical aesthetics, and literature from 1750 to the present. Publications include scientific articles on poetic and rhetorical language and its cognitive, emotional, and aesthetic effects, and monographs on the aesthetics of disgust and on Immanuel Kant, Ludwig Tieck, and Friedrich Hölderlin. He is a member of the Berlin-Brandenburg Academy of Sciences and has received the "Premio Internazionale di Estetica" (2012) of the Società Italiana di Estetica.

VALENTIN WAGNer is a Senior Research Fellow at the Max Planck Institute for Empirical Aesthetics in Frankfurt, Germany. After completing his M.A. in Philosophy, he enrolled in a Doctoral Program in Psychology and wrote a Ph.D. thesis on lexical access in sentence production at the University of Leipzig, Germany. Since his postdoctoral tenure at the Cluster of Excellence "Languages of Emotion" (Freie Universität Berlin), his primary research interests have been aesthetics and emotions, specifically, the aesthetics of language and speech, the processes of language production and comprehension, mixed emotions, and the interplay of aesthetics and emotions.

Eugen Wassiliwizky has studied Psychology with the main emphasis on Cognitive Neuroscience, as well as Classics and Musicology, at Philipps University in Marburg, McGill University in Montreal, and the Max Planck Institute for Human Cognitive and Brain Sciences in Leipzig. His main research topics are aesthetic emotions - especially those experienced in response to poetry and films, with a particular focus on neural and physiological correlates of such emotional processes. Wassiliwizky has worked at the Neuropragmatics and Emotion Lab (McGill University) and the Cluster of Excellence "Languages of Emotion" (Freie Universität Berlin), and is currently a member of the research staff at the Max Planck Institute for Empirical Aesthetics in Frankfurt.

Thomas Jacobsen is a Professor of Experimental and Biological Psychology at Helmut Schmidt University/ University of the Federal Armed Forces Hamburg, Hamburg, Germany, is the author of publications in (neurocognitive) psychology, including auditory processing, language, empirical aesthetics, and executive function. He was a Visiting Scholar in Cognitive Neuroscience at the University of California, San Diego, fellow of two McDonnell Summer Institutes in Cognitive Neuroscience, and a Visiting Professor at the University of Vienna and the Freie Universität Berlin, and received the Alexander Gottlieb Baumgarten Award of the International Association of Empirical Aesthetics (IAEA) in 2008.
Julian Hanich is an Associate Professor of Film Studies at the University of Groningen in the Netherlands. His first monograph entitled Cinematic Emotion in Horror Films and Thrillers: The Aesthetic Paradox of Pleasurable Fear was published by Routledge in 2010. His second monograph The Audience Effect: On the Collective Cinema Experience was published by Edinburgh University Press in 2017. His articles have appeared in the journals Psychology of Aesthetics, Creativity and the Arts, Screen, Cinema Journal, Projections, NECSUS, Studia Phaenomenologica, Movie, Film-Philosophy, Jump Cut, and New Review of Film and Television Studies.

Stefan Koelsch is a Professor at the Institute for Biological and Medical Psychology at the University of Bergen (Norway). He holds master's degrees in Music, Psychology, and Sociology, and earned his Ph.D. and wrote his Habilitation thesis at the Max Planck Institute for Cognitive Neuroscience (Leipzig), where he also led the Independent Junior Research Group "Neurocognition of Music" after being a postdoctoral fellow at Harvard Medical School (Boston, MA). His research foci include the neurocognition of music, neural correlates of emotion, music therapy, similarities and differences between music and language processing, neural correlates of cognition and action, emotional personality, and the unconscious mind.

\section{Introduction}

Enjoyment associated with negative emotions in art reception has been a central issue in poetics and aesthetics ever since Aristotle's theory of tragedy (1961). Many plays, operas, poems, and films elicit feelings of sadness and melancholy, and horror movies are a very popular genre. Even the emotion of disgust plays an important role in contemporary art and entertainment, from photography and installation art to teenage comedies. As these examples indicate, our model of art reception is not limited to the highbrow arts, but applies to a broad range of media products, as well. Throughout this article, we use the term art in this broader sense.

Exposure to artworks is widely believed to be driven by hedonic expectations and actual hedonic reward (Arnold 1960; Berenbaum 2002; Dubé \& Le Bel 2003; KnoblochWesterwick \& Keplinger 2006; Zillmann 1988). Moreover, only positive emotions or, put more generally, only positive affect (for the distinction between emotion and affect, see Russell [2003] and Scherer [2005]) is known to support hedonic, or friendly, approach behavior, whereas negative affect primes avoidance, defensive action responses, or hostile approach behavior (Norris et al. 2010). Self-sought hedonic exposure to negative emotions in art reception has therefore come to be called a "paradox" (Hume 1757). This introduction outlines the model we have developed to solve this paradox (see Fig. 1). Previously proposed solutions are reviewed in detail throughout the pertinent sections of this article as we spell out the individual components of our model. In a nutshell, what distinguishes the model presented here from earlier efforts can be highlighted through three points: 
Menninghaus et al.: The Distancing-Embracing model of the enjoyment of negative emotions in art reception

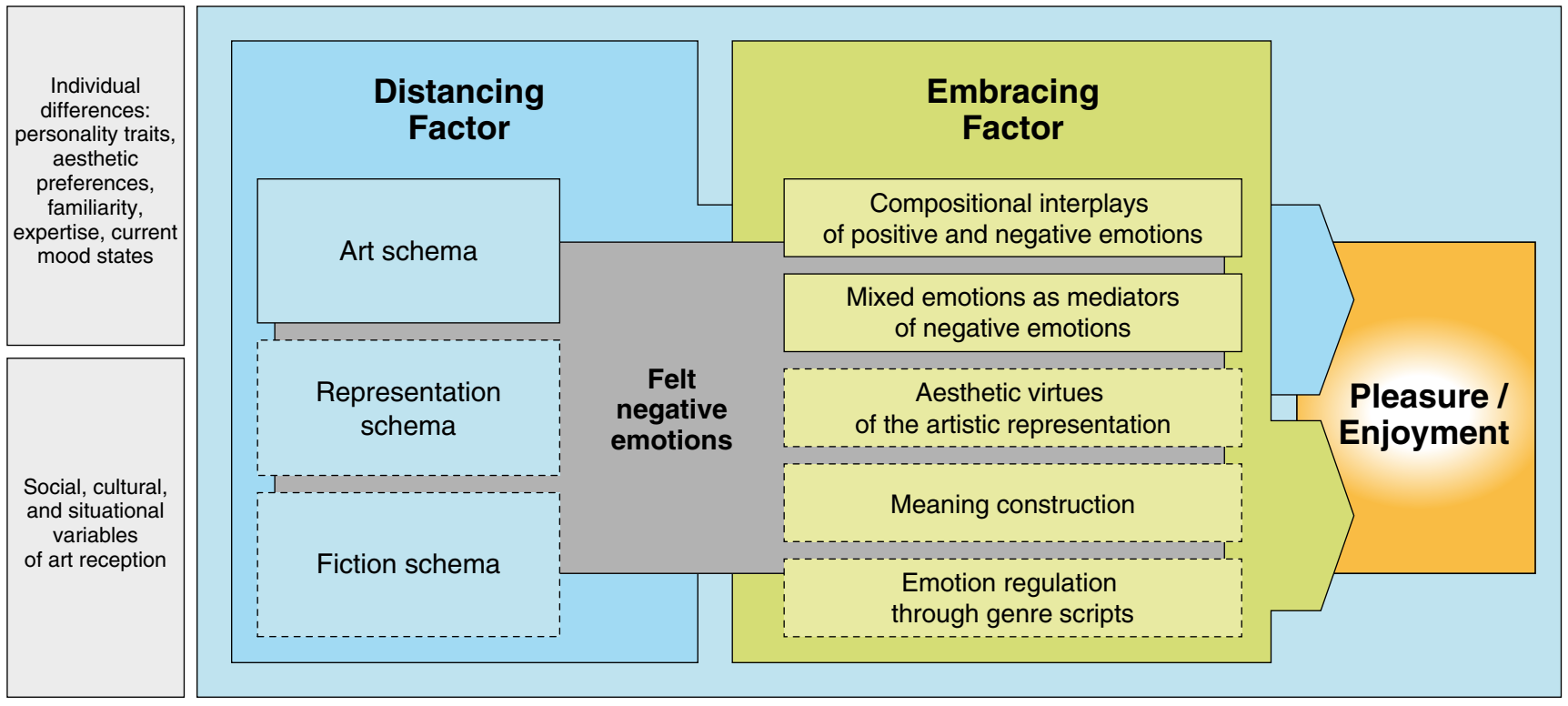

Figure 1. (Menninghaus et al.) The Distancing-Embracing model. Solid rectangles indicate the processing components that are always involved in making negative emotions enjoyable, whereas the dashed rectangles indicate components that are likely to be only occasional contributors to such enjoyment (for details, see sects. 3 and 4 of this article).

A. This is not a processing model for rare, exceptional cases in which enjoyment is associated with negative emotions. Rather, we suggest that negative emotions are quite generally a resource that is predestined for the arts' purposes. Section 2 derives this basic assumption from juxtaposing key findings of recent psychological research on negative emotions and key tenets of classical poetics. Psychological research suggests that negative emotions have a distinct potential for high intensity of subjective feeling, a powerful grip on attentional resources, and privileged storage in memory. Poetics suggests that these powers are precisely what the arts strive for. Hence, it appears that negative emotions and art reception may be a perfect match. Guided by this assumption, our model addresses the following questions: How can the arts adopt the particular powers of negative emotions to secure attention, intense emotional involvement, and high memorability without recipients experiencing the nonhedonic consequences of negative affect? Moreover, does negative affect also contribute to enjoyment because it is negative affect, and not just as a promoter of high emotional intensity and memorability? If so, which processing components and/or mechanisms support such hedonic benefits of experiencing negative affect in art contexts?

B. Responding to these questions, our model proposes two complementary processing factors, each of which includes several components. The first, already fairly well researched group of components (the "Distancing" factor) consists of the cognitive schemata of art, representation, and fiction. Situational activation of these schemata precedes the online processing and is maintained throughout it. (Reflecting this assumption, the color assigned to the Distancing factor in Figure 1 is also a background color for the Embracing factor and for Enjoyment.) The art, representation, and fiction schemata modify several important appraisal dimensions of negative emotions. As a result, they keep negative emotions at some psychological distance, thereby safeguarding the hedonic expectations of art reception against being inevitably compromised by the experience of negative emotions.

On this basis, the second group of processing components (the "Embracing" factor) positively integrates, assimilates, or adopts the powers of negative emotions in the service of making art reception more emotional, more intense, more interesting, and, in the end, more rewarding. This factor, which is the prime focus of the present article, consists of five components. Whereas the activation of particular acquired genre scripts mostly precedes the online processing and is analogous to the top-down cognitive activation of the situation schemata of the Distancing factor, the other four components are operative during online processing, with the component of meaning construction being the only component that can well be operative past the end of the actual exposure. Here is a brief description: (1) compositional interplays of positive and negative feelings are hypothesized to render art processing richer in emotional variation and less prone to induce boredom than types of pleasure that involve exclusively positive feelings; (2) concomitant mixed emotions are hypothesized to serve as bipolar mediators for incorporating negative emotions into positive enjoyment; (3) aesthetic virtues of the artistic representation itself (for instance, the beauty of the wording, musical sound, or painterly execution in terms of color, shape, and abstract patterns) promote dimensions of liking and enjoyment that are based primarily on lowlevel perceptual processing, thereby creating a (more) positive environment for the processing of concomitant negative emotions; (4) processes of (symbolic) meaning construction can redeem negative emotions on the level of higher cognitive processes; and (5) the emotion-regulatory implications of particular acquired genre scripts, such as the power of the normative happy end of (prototypical) fairy tales, allow readers/listeners to go through the preceding dire situations of need and conflict in a less desolate way 
Menninghaus et al.: The Distancing-Embracing model of the enjoyment of negative emotions in art reception

than could be expected in the absence of an established mental model of a fairy tale.

Thus, the model includes eight components that recruit a variety of cognitive and perceptual processes at different time points before, during, and after the exposure; some of these components are likely to be operative simultaneously during online processing. All of these components are hypothesized to exert specific emotion-regulatory/transformative effects on the processing of negative emotions. Notably, regardless of the assignment of the eight components to different time frames, our multicomponent model is not a component-process model. A detailed process model could not possibly cover music, literature, and the visual arts at the same time. After all, existing process models are invariably and for good reasons confined to particular art domains (Brattico et al. 2013; Jacobs 2015; Juslin 2013; Leder et al. 2004; Pelowski et al. 2016). The present article aims, however, precisely at identifying processing components that are hypothetically relevant for the hedonic processing of negative emotions across the art domains of music, literature, and - with a limitation mentioned at the very end of this Introduction -the visual arts.

Our Distancing-Embracing model shares with existing comprehensive models of art reception the general assumption that all aesthetic appreciation involves interactions of person variables; situational, cultural, and historical context; as well as stimulus characteristics (Bullot \& Reber 2013b; Chatterjee 2013; Jacobsen 2006; Leder et al. 2004).

C. In the existing literature, the eight components of our two-factor model have been discussed largely as alternatives (to the extent that the individual components were actually discussed at all). In contrast, our model stipulates that at least one component of the Distancing factor and one component of the Embracing factor need to be combined to satisfactorily explain the pleasure in art reception that is associated with negative emotions. Indeed, in most cases, two, three, or even all five components of the Embracing factor are likely to play a role.

All eight components of our model identify features that distinguish the experience of negative emotions in art reception from their analogues in real-life contexts. Based on some of these components - most notably, the cognitive art and fiction framing-philosophers have suggested conceiving of art-elicited emotions as "make believe," "as if," or "quasi" emotions (Gaut 2003; Levinson 1997a; Mulligan 2009; Skulsky 1980; Solomon 2003). Despite the partial overlap with our argument, we refrain from using any of these terms for two reasons. First, by their very linguistic nature and hence irrespective of how they are defined by individual authors - these terms evoke the notion (which we consider misleading) that art-elicited emotions may be somehow a species of inauthentic emotions. Second, the nuances of meaning distinctive of the above-quoted terms are highly debated even within the philosophy of emotions and barely known beyond this field. Therefore, projecting the eight processing components along which we distinguish art-elicited and ordinary emotions onto the above-quoted terms did not seem a promising effort for the purposes of the present article.

The present article focuses entirely on the core components of our model. Other important variables that are represented by the left-hand side of Figure 1, specifically, individual, historical, and cultural differences that are likely to influence the enjoyment of negative emotions in art contexts, lie beyond the scope of this article. Moreover, because our model exclusively accounts for the immediate experiential correlates of exposure to artworks that elicit negative emotions, we likewise do not discuss short-, medium-, or long-term functional benefits for psychological well-being that might be served by experiences of this type, such as catharsis (Aristotle 1961), exercising and developing one's cognitive and affective capabilities (Bloom 2011, p. 173; Kidd \& Castano 2013; Kumschick et al. 2014; Nussbaum 2008), vicarious acting, coping with fears and terror management (Goldenberg et al. 1999), and homeostatic regulation (Sachs et al. 2015).

Notably, treatises on literature were the first to stipulate that their compositional trajectories should go through a broad variety of stages and highly varying emotional implications (cf. Menninghaus 2003). In line with this tradition, recent empirical research into the enjoyment of negative emotions in art reception also focuses preeminently on the arts that unfold in time, specifically, film, music, and literature. Temporal artworks require far longer exposure times and attention spans than purely spatial objects, such as painting and sculpture; accordingly, composition-guided emotional variation over longer periods tends to be greater in these temporal arts. Given that the present article not only proposes novel designs for empirically testing our hypotheses, but even more strongly also relies on reviewing available empirical evidence, we cannot but share this primary focus on the temporal arts. Still, we also include the relatively few studies on negative emotion processing in response to visual artworks in our treatment of the topic. Clearly, to the extent that photographs, paintings, and statues tell a story (cf. Picasso's Guernica) or represent objects or scenes of negative emotional valence, they also allow for investigating aesthetic trajectories that involve negative emotions (cf. Gerger et al. 2014). At the same time, we do by no means rule out that visual representations of beautiful humans, animals, landscapes, stills, and so forth can well be enjoyed as beautiful without necessarily co-evoking any negative emotional associations. In any event, systematic comparisons across all art domains, as well as across different types of presentation (live vs. media-based), are clearly called for to determine the extent to which our model fits all individual art forms equally well.

\section{The goals of art, characteristics of negative emotions, and their strong role in artworks}

Ever since Greek and Latin antiquity, treatises on rhetoric and poetics widely assume that artworks compete for attention, intense emotional involvement, and high memorability (Lausberg 1998; Quintilian 1920). Recent psychological research has provided evidence that many negative emotions have a particularly powerful grip on attentional resources, are intensely felt, are less prone to fade or return to baseline level than positive emotions, and often have access to privileged storage in memory (Cacioppo \& Gardner 1999; Frijda 1988; Larsen \& Prizmic 2008; Musch \& Klauer 2003; Rozin \& Royzman 2001; Vaish et al. 2008). The formula "bad is stronger than good" (Baumeister et al. 2001) provocatively summarizes this line of research. Putting together these strands of rhetoric/ poetics and psychological theory, we conclude that negative emotions may actually not be an exceptional phenomenon 
Menninghaus et al.: The Distancing-Embracing model of the enjoyment of negative emotions in art reception

licensed only in special art genres, but rather a key resource to be drawn upon by many, if not most, artistic efforts.

Conforming to this reasoning, the representational arts, particularly those based on narrative plot, routinely involve social conflicts and both represent and elicit negative emotions in response to such conflicts (Grodal 2007; Krämer \& Witschel 2010; Scalise Sugiyama 2005). For dramas, novels, an epic poems this is true to an intriguing degree: Whereas failing marriages, unhappy love, long separations, adultery, betrayed friendship, and the like are routinely represented in great detail, we know of no novel that extensively depicts 20 years of happy married life, let alone the lifelong happy marriage of an elderly couple. Moreover, because the composition of narratives of all kinds is typically far more condensed than the unfolding of real-life events over a longer trajectory (Mar \& Oatley 2008), the underlying conflicts and corresponding negative emotions tend to become more pronounced in artistic representations. Poems, too, thrive at least as often on sad or even desperate feelings of uncertainty or negative certainty regarding the responsiveness of the beloved rather than on happy feelings of having one's love reciprocated. Correspondingly, Batteux noted in his influential treatise The Fine Arts Explained through a Single Principle: "Artists succeed much more easily with unpleasant objects in the arts than with pleasant ones" (1746/2015, p. 48). To be sure, many comedies and popular novels are precisely about happy love relations, yet they, too, typically fade out - just like all fairy tales invariably do-once a state of happiness has been reached, and do not depict the affectively positive states in nearly as much detail as the preceding states of uncertainty, conflict, and suffering.

Some authors have discarded the notion that artworks in fact involve recipients in negative emotions. They stipulated that recipients may only erroneously report experiencing (expectable) negative emotions in response to artworks with negative emotional implications, but actually not feel any relevant level of such emotions (cf. Kivy 1991, Ch. 8; Krämer \& Witschel 2010) because exposure to artworks is categorically different from ordinary "real-life" contexts. The majority of the studies on responses to horror films (Andrade \& Cohen 2007), sad music (Vuoskoski \& Eerola 2012), sadly moving films (Hanich et al. 2014), and disgusting images (Wagner et al. 2014) have relied exclusively on subjective self-reports and, therefore, cannot rule out this possibility. However, several studies on sad music (Lundqvist et al. 2009), affectively negative pictures (Gerger et al. 2014), and anger-inducing performances (Wagner et al. 2016) have also reported objective physiological responses of the autonomic nervous system (ANS) that were in line with the patterns expectable for genuine episodes of the respective negative emotions. All of these studies converge in showing (a) that negative emotions are not just represented in plots and expressed by characters, but are also, at least partly, felt by audiences (cf. also Juslin 2013; Mills 1993), and (b) that such felt negative emotions appear to covary with aesthetic liking and positive enjoyment.

Regarding instrumental music, the historical and theoretical discourse is far less focused on the apparent paradox of negative emotions than it is for literature. Many empirical studies emphasize the role of positive emotions (Blood \& Zatorre 2001; Hunter et al. 2008; 2010; Keller \& Schubert 2011; Salimpoor et al. 2011; Schellenberg et al. 2008; Witvliet \& Vrana 2007; Zentner et al. 2008). Still, negative emotions, especially sadness, have also been reported to be both expressed by purely instrumental music and felt by listeners (Hunter et al. 2010; Schellenberg et al. 2008), and there is substantial evidence that music-elicited feelings of sadness correlate positively with liking the pertinent pieces of music (Garrido \& Schubert 2011; Panksepp 1995; Panksepp \& Bernatzky 2002; Schellenberg et al. 2008; Schubert 2013; Taruffi \& Koelsch 2014).

Research into different types of pleasure has shown that intellectual and emotional pleasures (including those of art reception) routinely "encompass negative emotions like sadness ... and positive emotions that entail complex appraisal" (Dubé \& Le Bel 2003, p. 291; see also Berenbaum 2002). In contrast, physical pleasures tend to be least affected by concomitant negative emotions (Dubé \& Le Bel 2003). This provides another piece of evidence in favor of the assumption that the inclusion of negative emotions should not be considered an exceptional case of art reception, but rather constitutes a general distinctive trait of it.

But do artworks that elicit higher levels of negative emotions indeed support more intense emotional responses and are also more memorable than artworks not involving negative emotions, or involving them only to a lower degree? No such comparisons have been performed to date in any systematic fashion. Still, throughout this article, we report individual pieces of evidence in favor of this assumption. To start with, we refer to a particularly conspicuous example. Not coincidentally, it comes from research into highly intense emotional responses to artworks: those associated with chills and goosebumps (Benedek \& Kaernbach 2011; Blood \& Zatorre 2001; Goldstein 1980; Grewe et al. 2007; Panksepp 1995; Rickard 2004; Salimpoor et al. 2011; 2009). Activation of chills and goosebumps in listening to music or watching a film is accompanied both by increased electrodermal activity as indicative of emotional arousal of the autonomic nervous system and a strong activation of the primary reward network (Blood \& Zatorre 2001; Salimpoor et al. 2011); physiological arousal reaches maximal levels when goosebumps co-occur with emotional tears (Wassiliwizky et al. 2017a). Importantly, recent research has shown that chills and goosebumps in art contexts are highly likely to be not just "peak emotional responses" of an unspecific nature, but physiological correlates of states of being emotionally moved (Benedek \& Kaernbach 2011; Wassiliwizky et al. 2015). This emotion state, in turn, has been shown to routinely involve interplays, or blends, of positive and negative emotional ingredients, not just in its sad, but also in its joyful variant (Menninghaus et al. 2015b).

Moreover, in a free-recall task in which participants were asked to remember feelings of being moved in response to real-life and fictional events (the latter being nearly always related to artworks), episodes of being joyfully moved were far more frequently recalled for the real-life memories than episodes of being sadly moved; inversely, memories related to fictional artworks showed a strong bias toward the sad variant of being moved (Menninghaus et al. 2015b; for more details on sadness and being moved, see sect. 4.2.1). Sadly moving films and narratives were also rated as affecting the audience more intensely than joyfully moving ones (Goldenberg et al. 1999; Menninghaus et al. 2015b; Wassiliwizky et al. 2015). Finally, experiencing chills and goosebumps in emotionally moving art context also has strong memory effects: Many recipients not only remember which artworks elicited these responses; they 
Menninghaus et al.: The Distancing-Embracing model of the enjoyment of negative emotions in art reception

even remember the specific moment in the respective artworks that repeatedly elicited them (Blood \& Zatorre 2001; Panksepp 1995; Salimpoor et al. 2011; Sumpf et al. 2015). Hence, these emotional responses can be considered as prime examples of high emotional intensity, high levels of aesthetic enjoyment, and high memorability as associated with artworks, the processing of which includes marked levels of negative affect. (Because the respective studies relied largely on participants' choices of artworks based on their memories of prior exposure, they moreover show that the power of familiarity to predict aesthetic liking [Bornstein 1989; Reber et al. 1998; Zajonc 1968] also extends to cases in which aesthetic liking is specifically associated with concomitant negative feelings.)

In a similar vein, the peak-end rule-according to which the mean of peak and end affect is a better predictor of post hoc liking ratings for an affective episode than the average of all continuous ratings (Do et al. 2008; Geng et al. 2013; Kahneman et al. 1993) - has been successfully applied to art reception in a way that implicitly relies on the very hypothesis we advocate here. The respective studies (Rozin et al. 2004; Schäfer et al. 2014) collected peak and end ratings not for pleasure and/or displeasure, positive and/or negative affect, but for intensity and, hence, for an experiential quality that applies equally to positive and negative affect. This choice of rating item implies the notion that not only positive, but also negative affect can equally contribute to overall liking if, and to the extent that, it supports felt emotional intensity.

Summing up, important characteristics of negative emotions can be theoretically conceived as predestined resources for the artists' efforts to produce artworks that powerfully and pleasurably affect recipients. A survey of the plots of literature, of research on music, and of studies on intellectual pleasures altogether supports the notion that negative emotions are not a liability or an exceptional license, but rather a resource on which these pleasures rely. Moreover, there is evidence that negative emotions are not mere misattributions, but that recipients actually feel them and that this is beneficial rather than detrimental for the intense emotional involvement, the highly rewarding quality, and the high memorability of art reception. At the same time, the emotional powers of negative emotions are by their very definition tied to experiencing negative affect and not just affective intensity. Thus, access to the distinctive art-compatible powers of negative emotions does come at a price, and the question arises how the arts can reap the benefits of negative emotions without compromising their ultimate goal. Our response to this question is the Distancing-Embracing model as developed in Sections 3 and 4.

\section{Cognitive mechanisms that keep negative emotions at a psychological distance: The Distancing factor}

The present section spells out the first pillar of our model as outlined in Figure 1: the Distancing factor. Specifically, we focus on the effects of the situational activation of three cognitive schemata or frames: those of art, representation, and fiction (for the schemata and cognitive framing theory in general, see Abelson 1981; Brewer \& Nakamura 1984). We discuss evidence for the following hypothesis: Activation of these cognitive schemata/frames should keep felt negative emotions at some psychological distance (= primary effect) and thereby open experiential spaces in which negative emotions are not inevitably incompatible with art-specific expectations of hedonic reward (= secondary effect). Following the construal-level theory of psychological distance (for this concept, see Trope \& Liberman 2010; Trope et al. 2007), we assume that the baseline, or reference point, for psychological distance is the art recipient's "me" in the "here" and "now" and that the various dimensions of psychological distance discussed below tend to be similar in effect (see also Cupchik 2002; Hanfling 2000).

Because the cognitive schemata of representation and fiction can be activated far beyond the confines of art, we define the three schemata under consideration separately. Notably, the three schemata are not mandatory for all arts: Instrumental music is mostly neither fictional nor representational, and the same holds for other forms of art such as abstract paintings and many experimental films. By contrast, the cognitive schema for reading literature and viewing/hearing films, theatrical plays, and operas typically simultaneously encompasses the schemata of art, representation, and fiction.

\subsection{The art schema}

Art reception typically implies an ongoing situational awareness that one is reading a book or watching a movie or listening to a piece of music rather than being involved in ordinary action or communication contexts (for applications to art contexts see Brewer \& Lichtenstein 1982; Dixon \& Bortolussi 2009; Hoeken \& Van Vliet 2000; Leder et al. 2004; Magliano et al. 1996; Mandler 1984; Schubert 2016; Visch \& Tan 2008; Zwaan 1994). This situation concept includes the notion of personal safety, because nothing represented on stage or in a book can directly harm viewers and readers (but see Hanich 2014). Moreover, readers, listeners, and viewers are in control of the situation in that they typically both seek out and continue or discontinue their exposure to a work of art in a self-motivated way (Andrade \& Cohen 2007; Apter 1992; 1993; Bartsch et al. 2008; Bloom 2011, p. 196; Rozin et al. 2013; Tan 2008). This control thesis (cf. Eaton 1982; Morreall 1985; Witasek 1904, pp. 116-17; for a critique see Iseminger 1983; Packer 1989; Smuts 2009a) is in line with experimental findings that tolerance for pain is substantially higher if one has the power to stop a painful treatment at one's will and, thus, is in control of the situation (Litt 1988).

Projecting the features of safety and control onto a cognitive appraisal account of emotions (Scherer 2005), all emotions experienced in an art-framing context should be more "self-intended" than analogous emotions in a different framing, because they are typically intentionally selfsought. Moreover, art-elicited emotions should entail little direct personal goal relevance and goal conduciveness for immediate practical purposes (Hunter \& Schellenberg 2010; Scherer 2012) and should not challenge our coping potential (apart from demands of cognitive understanding) or stimulate strong action responses (cf. Meinong 1902/ 1977; Scherer 2005). All of these cognitively distinctive features of art-elicited negative emotions should be reflected in how these emotions are subjectively experienced (Scherer 2005; Scherer et al. 2006) and should specifically work in favor of reducing their potential adverse effect on 
Menninghaus et al.: The Distancing-Embracing model of the enjoyment of negative emotions in art reception

hedonic processing. Thus, the psychology of emotions clearly predicts differences in emotional responses dependent on the ontological nature of the emotion elicitors. Philosophical theories have discussed comparable distinctions (Gaut 2003; Levinson 1997b; Mulligan 2009; Skulsky 1980; Walton 1990).

\subsection{The cognitive schema of representation: Effects of temporal, spatial, and cultural distancing}

Representations typically refer to events or scenarios that are not co-extensive in time and/or space with what they represent, with live media coverage overcoming the distance in time, but not in space. Moreover, representations typically highlight some of the perceptual features of an event at the expense of others, and writing even suppresses all direct visual, acoustic, and olfactory input features of the real or fictional events to which it refers. Once the temporal and/or spatial distance becomes large enough, another factor almost invariably comes into play, namely, the cultural difference of contexts. Thus, representations support only distanced, indirect, and -compared with their real antecedents -incomplete exposure. This should, in principle, work in favor of a psychologically more distanced response, as predicted by the construal-level theory of psychological distancing (cf. Trope \& Liberman 2010; Trope et al. 2007). For example, a temporal distance of only a few weeks has been shown to make it far more likely to find humorous aspects in the media coverage of a deadly hurricane (McGraw et al. 2012; 2014).

\subsection{Distancing effects as a result of fictional status}

A further dimension of distancing is exclusively involved in a subgroup of representations, namely, fictional (vs. factual) representations, including fictional artworks. The ontological shift into the realm of fiction has long been considered to work in favor of greater tolerance for and enhanced enjoyment of artworks that elicit negative emotions (cf. de Fontenelle 1692/1968; Zelle 1987, p. 162). Cognitively framing an act of murder or a scene of violent mutilation as fictional provides an awareness that no real person (or animal) has been physically harmed; this should alter emotional responses in comparison to witnessing comparable real acts. To be sure, the arts clearly thrive on the human propensity not to consistently maintain a clear-cut distinction between imagination, fiction, and belief systems, on the one hand, and reality, on the other (cf. Bloom 2011, pp. 155-76). However, even when we are immersed or absorbed in a story world (Busselle \& Bilandzic 2008; Green et al. 2008; 2012; Kuijpers et al. 2014; Zwaan 1999), our mental situation model is likely to retain at least some background awareness of this important ontological distinction (Tan 2008). This may, however, apply to children to a lesser degree (Weisberg et al. 2013).

In most cases of narrative artworks and media products, the fiction framing is likely to be largely co-extensive with the appraisal profile of the art framing sketched above. However, studying nonfictional art, non-art fiction, and fake documentaries (such as Orson Welles's radio production based on H. G. Wells's The War of the Worlds, or The Blair Witch Project) could yield additional interesting perspectives on both the overlaps and the differences between the art-versus-reality framing, on the one hand, and the fiction-versus-reality framing, on the other. There are even clear cases of fascination when viewing live footage of real disastrous events (such as the eruption of volcanos, major tornados, tsunami waves, plane crashes, and other accidents) and gigantic crimes (such as the attack on the Twin Towers in 2001), with no temporal, cultural, or art- or fiction-based distancing mechanisms and real humans involved (cf. Rimé et al. 2005). Documentary tragedies that go beyond mere live coverage of disastrous events have likewise been argued to yield some sort of pleasure, at least if a number of constraints are met (Friend 2007). In any event, even in live footage, where no distancing effects of fiction and temporal distance are involved, some spatial distance - sufficient to support the precondition of the viewer's personal safety - is still required, and the media-transmitted sensory impression (loudness, olfactory sensations, etc.) is also different from experiencing, for example, a real earthquake.

\subsection{Empirical evidence}

Whereas we theoretically introduced the art, representation, and fiction schemata as distinct components of our model, we review the pertinent empirical evidence conjointly. We do so because the few available studies on the distancing effects of the cognitive art schema mostly draw on representational and typically also fictional arts, thereby ruling out a strict separation of the three cognitive schemata. Some of the assumptions outlined in sections 3.1 through 3 have been tested in empirical studies. Episodes of being sadly moved when responding to fictional events have been shown to score significantly higher for the appraisals "self-sought," "self-caused," and "power to modify consequences" and lower for the appraisal "undesired outcome" than episodes of responding to real events (Menninghaus et al. 2015b).

A study that presented photographs of disgusting matter as either art photography or documentary photographs made for purposes of hygiene instruction found higher levels of positive affect in the art-framing group (Wagner et al. 2014); at the same time, reported feelings of disgust did not differ between the two conditions. Accordingly, a study on perceived sadness and anxiety in a fictionversus-nonfiction framing did not find any differences for the two conditions (Goldstein 2009). This suggests that the distancing effect of the art framing does not convert, let alone erase, negative emotional responses and need not even reduce the felt intensity of these responses to make them (more) compatible with positive enjoyment. Similarly, a study on moral feelings (McGraw \& Warren 2010) reported that a psychologically distancing framing rendered a moral violation (a man having sexual intercourse with a chicken) more benign and even somewhat amusing, even though feelings of disgust remained at the same level as for participants who were not primed in a psychologically distancing manner.

Using both pictures of the International Affective Picture System and genuine artworks and targeting a variety of both positive and negative emotions, a study by Gerger et al. (2014) yielded similar results. Although negative valence ratings did not differ for the two framings, pictures of negative valence were again more aesthetically liked in the artframing condition. Another study (Wagner et al. 2016) reported an analogous positivity/enjoyability effect of an art versus non-art framing for an elaborate identical anger- 
Menninghaus et al.: The Distancing-Embracing model of the enjoyment of negative emotions in art reception

inducing treatment of participants. Earlier studies had already shown similar effects of cognitive framings on emotional processing (Lazarus 1964; Lazarus \& Alfert 1964; Lazarus et al. 1962; 1965; Legrand \& Apter 2004). However, these studies did not specifically investigate the role of an art versus non-art framing, but of other mental framings. Finally, a recent electroencephalography study (Van Dongen et al. 2016) yielded neuroscientific evidence for implicit emotion regulation activated through an art framing.

\subsection{Downsides of emotional distancing mechanisms}

Classical aesthetics suggests that emotional distancing may be disadvantageous for positive emotional content: "The effects of imitation, so advantageous for unpleasant objects, work completely against pleasant ones for the same reason. The impression made by art is weakened .... Thus, all other things being equal, the heart must be much less satisfied by pleasant objects in the arts than it is by unpleasant ones" (Batteux 1746/2015, p. 48). For example, regarding beautiful humans or landscapes, it may in many cases be more pleasurable to behold the real objects rather than their painterly representations. Being part of a joyful festive event may also produce more intense joy than reading a description or looking at well-taken photographs of the event. Correspondingly, photographs of persons and genuine visual artworks that were both perceived as positive in affective valence received lower ratings for positive valence when presented in an art framing compared with a reality framing (Gerger et al. 2014); at the same time, aesthetic liking ratings were at the same level for both framings. Only the pictures with negative emotional valence profited from the art framing: They received significantly higher liking ratings, even though the negative valence ratings and facial electromyography-based measures of positive and negative affect remained virtually unchanged. Thus, Batteux was right: The art framing, as compared with the non-art framing, yielded asymmetrical effects on pictures of positive and negative valence such that the "impressions made by art" were more positively appreciated in the case of pictures of negative valence.

Future research might investigate whether, and to what extent, different dimensions of distancing - spatial, temporal, historical, symbolic, fiction based-exert different effects on negative emotion processing, the extent to which these effects are cumulative, and whether there are ideal levels of distancing as compared with over- or underdistancing (cf. Bullough 1912; Scheff 1979, Ch. 5).

\section{How the arts of selecting, combining, and formally elaborating constituent parts of artworks promote the enjoyment of negative emotions: The Embracing factor}

Psychologically distancing effects as a result of the cognitive schemata of art, representation, and fiction do not by themselves explain why the arts might not be better advised to wholly avoid negative emotions and to focus exclusively on beauty and concomitant positive emotions. The activations of these cognitive situation schemata exclusively secure preconditions for the enjoyment in question.
Additional psychological mechanisms of a more positive enabling nature are needed that take advantage of this precondition and actually adopt the powers of negative emotions for pleasurable purposes (cf. Andrade \& Cohen 2007). The present section introduces and discusses the five processing components that make up this positive Embracing factor of our model.

Projected onto the framework of poetics (Lausberg 1998; Quintilian 1920), the first two components bear on the dimensions of inventing/selecting the constituent parts of an artwork (inventio) and combining them into a well-composed temporal or spatial order (dispositio). The third bears on the material execution of artworks in terms of wording (elocutio), sound patterns, coloring, and so forth. A fourth component (which hermeneutics added to the system of poetics) concerns processes of seeking or constructing meaning (interpretation) in response to artworks. The fifth component is another classical aspect of poetics, namely, the emotion-regulatory power of specific acquired genre scripts, that is, the anticipatory adjustment of our emotional expectations and processing routines depending on whether we read a text known to be a tragedy, a fairy tale, a satire, or some other genre.

\subsection{Interplays of positive and negative emotions}

In this subsection, we advocate the following hypothesis: Composition-driven trajectories of aesthetic processing (cf. Fitch et al. 2009) that involve negative emotions are conducive to enjoyment because the pleasure taken in the beautiful representation of wholly positive and beautiful objects and narratives tends to be less intense, profound, and self-supportive and more prone to induce boredom than pleasure that includes a dynamic interplay of positive and negative emotional responses. An earlier version of this hypothesis was put forward by Kant: "What makes theater plays (whether tragedies or comedies) so enticing? The fact that certain difficulties emerge in all of them - anxiety and perplexity between hope and joy - so that the interplay of opposite feelings sets the mind of the spectator in motion" (Kant 1798/1996, p. 232, emphasis added). Other treatises of classical philosophical aesthetics have similarly argued that interplays of positive and negative feelings are aesthetically superior to a purely and thoroughly positive affective content and tonality (Mendelssohn 1759/1991; 1761/1997, p. 143; Wezel 1785/1971).

Zillmann's (2006) theory of dramatic plot and, specifically, of the "excitation transfer" from the peak moment of threat and uncertainty to the peak moment of a happy end can be understood as a genre-specific variant of the general hypothesis of a pleasurable interplay of positive and negative emotions. Solomon's opponent-process theory even proposes that not just artworks, but all emotion-eliciting events give rise to both negative and positive (i.e., opposite) affective processes (Solomon 1980; Solomon \& Corbit 1974). As a consequence of its generality, this theory is, however, as much designed to account for maladaptive (drug addiction) as for hedonic outcomes of such opponent processes. Still, the affective trajectory of parachute jumping as conceptualized by Solomon (1980) is a good non-art example of a trajectory in which negative affect (temporary anxiety) is more than counterbalanced by a state of relief, accomplishment, and even euphoria, which, in turn, is energized by the preceding components 
Menninghaus et al.: The Distancing-Embracing model of the enjoyment of negative emotions in art reception

of negative affect. Notably, however, Solomon himself never followed up on remarks that art reception might be a good test case of his domain-independent model, too (Rozin 1999). In fact, he even speculated that "perhaps some aesthetic pleasures have no opponent process" (Solomon \& Corbit 1974, p. 142). In any event, because Solomon's theory temporally separates hedonic and aversive episodes as first and "after" processes, it does not entail provisions for genuine co-activations of positive and negative affect (cf. Andrade \& Cohen 2007).

The compositional rule of going through emotional antitheses is based on the assumption that the inclusion of "unpleasant feelings" provides aesthetic trajectories with a greater affective amplitude, emotional depth, and rate of dynamic change. A series of purely "pleasant feelings" is held to be aesthetically inferior to this interplay, because it is hypothetically limited in its capacity to support intellectual interest over longer trajectories and consequently more prone to wear out. Prominent terms that designate profoundly negative responses to too positive or even sweet content and to too much unadulterated beauty in art reception are "boredom," "satiation," and even "satiation-driven disgust," as first discussed by Mendelssohn and Kant (cf. Menninghaus 2003). One of the most fundamental principles of aesthetics - the rule of uniformity amidst variety advocated by virtually all eighteenthcentury aesthetics, as well as by Fechner (1876) and Berlyne (1971a; 1974) - supports this assumption, as well: If richness in "variety" is a preeminent feature of aesthetically appealing stimuli, then variety should also apply to the emotional aspects and effects at which they are aimed, with "variety" in emotional effect requiring the inclusion of negative emotions. These considerations suggest that negative emotions are conducive to enjoyment not just because their negative affective nature is outbalanced by their effects on the intensity of emotional involvement, but also because this negative affective nature acts as a remedy against aesthetic failures that could result from artistic compositions that rely exclusively on positive affect. In this capacity, the contribution negative emotions make to pleasurable interplays of positive and negative emotions relies primarily on their very negative affective nature.

To test and further refine the hypothesis of this subsection, we propose experimentally investigating trajectories that feature varying proportions of positive and negative emotional ingredients. In all likelihood, increasing the share of negative emotions from near zero to ever higher levels will not monotonically increase aesthetic appreciation; rather, it is likely to show an inverted U-shape (see also Berlyne 1971a; 1974). To test this hypothesis, we suggest developing fictional scenarios that manipulate negative and positive emotion cues in a systematic manner, ranging from solely negative or solely positive emotion cues to different admixtures of these. The scenarios that make use of only one type of emotion cue should receive comparatively low ratings on measures for aesthetic appreciation. Adding positive emotion cues to previously exclusively negative ones and adding negative cues to previously exclusively positive ones should, in both cases, result in higher scores on a variety of measures for aesthetic appreciation and emotional involvement (liking, beauty, interest, suspense, being moved, intensity of involvement, etc.). Studies of this type also have a potential to investigate upper limits for and optimal levels of the inclusion of negative emotions for pleasurable purposes, both in general and with respect to the different domains and genres of art. Speeches consisting wholly of epideictic praise (eulogy) may provide a good test case for a too exclusive focus on positive affect.

\subsection{Concomitant mixed emotions as mediators of negative emotions' positive contributions to enjoyment}

This subsection adds a third player to the interplay of positive and negative emotions as discussed in the preceding subsection, namely, mixed emotions. Based on a review of research on sad films and sad music, as well as on horror films, we advocate the following hypothesis: Concomitant mixed emotions serve as bipolar mediators for incorporating negative emotions into positive enjoyment. This hypothesis has no direct antecedent in classical poetics and aesthetics. It is based wholly on recent empirical research on the enjoyment of negative emotions (Hanich et al. 2014; Wassiliwizky et al. 2015), while in general informed by research on co-activations of positive and negative affect (e.g., Larsen et al. 2003). Specifically, we discuss evidence for the hypothesis that the principal mediator emotions for sad films and horror films are feelings of being moved and suspense, respectively. We argue that both mediator emotions are of a mixed emotional nature and that this facilitates their role in reconciling negative emotions with the hedonic expectations of art reception (for the concept of mixed emotions, see (Carrera \& Oceja 2007; Ersner-Hershfield et al. 2008; Larsen \& McGraw 2011; Oceja \& Carrera 2009; Rafaeli et al. 2007; Schimmack 2001). A study on benign moral violations (McGraw \& Warren 2010) has already shown that mixed emotional responses to these violations can be a way to retain feelings of moral rejection while simultaneously finding the violations amusing and, hence, enjoyable.

Whereas the interplay of positive and negative emotions discussed in the previous subsection relies on a series of affective antitheses (and the interaction between them), the present subsection addresses phenomena that in terms of rhetoric and poetics are metonymical in nature (cf. Jakobson 1973). Metonymies are based on contiguity relations, that is, the meaning of one element of a representation is strongly informed by a neighboring or co-occurrent element. For example, in a novel, the deranged state of a handbag can be described to shed light on the affective state of the person carrying it (who might otherwise successfully conceal his or her state of embarrassment). Regarding emotions, contiguity in affect space underlies typical "family resemblances" between individual emotions. Thus, feelings of being moved and of being touched cluster closely with feelings of sadness and nostalgia; moreover, all four of these emotion states frequently co-occur (Menninghaus et al. 2015b; Sedikides et al. 2008). The principle of metonymical transfer then predicts that the more unambiguously negative emotions (for instance, sadness) that are part of such contiguity-based clusters can profit from adjacent emotions that are more positive in affective nature (for instance, being moved and nostalgia). Similarly, at the opposite end of affect space, horror and suspense are both high-arousal emotions that frequently co-occur in response to specific genres of artworks and media products, yet they differ in affective valence: suspense is of a mixed affective valence (for details, see sect. 4.2.2), whereas horror is typically of an unambiguously negative nature. 
Menninghaus et al.: The Distancing-Embracing model of the enjoyment of negative emotions in art reception

The principle of metonymical contiguity then predicts that the more positive affective nature of suspense can inform and partly transform co-occurrent or adjacent feelings of horror and, hence, lead to perceiving horror as (more) enjoyable. Thus, in both cases, the arts take advantage of latent affinities between emotions that are contextually activated by means of compositional contiguity.

\subsubsection{The case of sadness}

For eighteenth-century Scottish moral sense philosophers such as Lord Kames (1751/2005; see also Zelle 1987, p. 176), feeling compassion for the plight of others conforms to the standards of moral virtue and humanity; those who experience these feelings can therefore be pleased with their own emotional responses (cf. Aikin \& Aikin 1773; Princess Elisabeth of Bohemia \& Descartes 1645/2007, p. 118; for a more recent philosophical version, see Feagin 1983; for a more recent psychological version, see Schaller 1993). In recent media psychology, this focus on compassion, or empathy (for subtle distinctions between these terms, see Klimecki et al. 2012) as self-gratifying prosocial responses to the suffering of others has been reformulated in the "meta-emotions" model (Bartsch 2007; 2008; Bartsch \& Viehoff 2003; Bartsch et al. 2008, 2010; Mills 1993; Oliver 1993; Schramm \& Wirth 2010). Typologically, the different versions of the hypothesis of enjoyment qua self-gratifying empathy suggest a transformation of sadness into a source of pleasure by means of a mediation, a detour through a concomitant emotion - in this case empathy/ compassion-together with a shift in focus from the object of the empathic response to the implication of this response for how the onlooker feels about him- or herself. Empirical evidence that the positive affect found in responses to sad films may actually be causally a result of such meta-emotional (re)appraisals rather than other response dimensions is, however, only tentative. Moreover, David Hume already offered a powerful argument against this hypothesis: If awareness of our own prosocial sympathetic impulses were by itself a sufficient reason for deriving pleasure from sad scenes, it would follow that "a hospital would be a more entertaining place than a ball" (Hume 2004, p. 243).

Another explanation stipulates that sad music, poems, narratives, and films can be enjoyed because, and to the extent that, they are blended with, or integrated into, episodes of being moved (for general definitions of this emotion state, see Kuehnast et al. 2014; Menninghaus et al. 2015b; Tokaji 2003) and/or nostalgia (Juslin 2013; Taruffi \& Koelsch 2014), and that this association allows sadness to partake in the mixed, yet predominantly positive affective nature that is characteristic of being moved (Hanich et al. 2014; Wassiliwizky et al. 2015) and/or nostalgia (Routledge et al. 2011; Sedikides et al. 2008; Wildschut et al. 2006; 2010).

Two prototypes have been shown to account for a great portion of episodes of being moved. In the sadly moving prototype, experiences of loss (separation, death) or acts of sacrifice are associated with a positive appreciation of the value and memory of a beloved and/or with feelings of love and/or empathy on the part of bystanders and onlookers (Menninghaus et al. 2015b; Tokaji 2003). Similarly, situations of bidding farewell or separation can be represented -and have been shown to be experienced as not just sad, but sadly moving (Wassiliwizky et al.
2015), because they co-activate both the pain of temporarily or permanently severing an important social bond and a heightened sense of the value of this bond and also, in part, hopes for restoring it. The second prototype of being moved is of a joyfully moving nature. Typical eliciting events include nostalgic memories of one's childhood or a former romantic relationship, as well as marriages, reunions, and reconciliations (Kuehnast et al. 2014; Menninghaus et al. 2015b; Taruffi \& Koelsch 2014). In all of these cases, the predominant positive feelings come with some negative antidotes, such as an awareness that the happy times of childhood are forever gone, that a happy reunion was preceded by a painful period of separation, or that a couple will invariably have to face days and experiences that are less joyful than their wedding day.

Regardless of the inverse proportions of sad and joyful feelings in the two prototypes of being moved, overall affective responses to emotionally moving artworks show a predominance of positive affect (Hanich et al. 2014). This implies that the partially bipolar nature of states of being moved does not turn them into examples of disconcerting ambivalence and, hence, examples of a response pattern that psychological research has closely associated with the very notion of mixed emotions (Larsen et al. 2001; Norris et al. 2010). Thus, concomitant negative memories of a previous separation typically do not turn a reconciliation into an event that is emotionally ambivalent or that calls for a difficult decision between embracing the positive and embracing the negative response dimensions. Moreover, both the sad and the joyful/happy variants of being moved virtually always include more or less oblique references to positive feelings of bonding and attachment and to prosocial norms, values, and self-ideals (Fukui \& Toyoshima 2014; Konečni 2005; Konečni et al. 2007; Menninghaus et al. 2015b; Panksepp 1995; Stel et al. 2008; Tokaji 2003).

These findings entail two further insights into the art of selecting and combining negative emotion cues. First, for all of their reliance on sadness, emotionally moving artworks by no means draw on all possible instances of sadness. Rather, only fairly circumscribed varieties of sadness are eligible to be associated with feelings of being moved. For example, forgetting one's favorite jacket in a restaurant and not retrieving it another day can elicit regret, and even some anger, about one's own forgetfulness and may later lead to feelings of a saddening loss; however, this type of sadness is not likely to elicit feelings of being emotionally moved. Briefly put, only sad feelings that have a direct bearing on social bonding and attachment can become ingredients of feelings of being moved (Menninghaus et al. 2015b); sentimental and nostalgic feelings place similar constraints on including ingredients of sadness (Sedikides et al. 2008; Wildschut et al. 2006). Hence, possible incorporation into altogether pleasurable mixed feelings does not extend to all instances of sadness, but is subject to selective constraints. We therefore propose testing with a broader corpus of "sad" artworks the hypothesis that a great share of sad feelings elicited by artworks cluster closely with feelings of being moved, attachment feelings, nostalgia, sentimental feelings, and feelings of quiet (the latter because sadness is low in arousal; cf. Kreibig 2010; Russell 2003).

Most importantly for the present context, feelings of being moved by sad film clips show a robust positive correlation with appreciating the respective films as artistic 
Menninghaus et al.: The Distancing-Embracing model of the enjoyment of negative emotions in art reception

achievements and with the level of enjoyment they elicit (Hanich et al. 2014; cf. also Tokaji 2003). In contrast, the positive correlation between felt sadness and aesthetic appreciation/enjoyment disappears when potential mediating effects of feelings of being moved are controlled for. Wassiliwizky et al. (2015) replicated this finding and extended it by showing that no such mediation was found for joyfully moving films; felt joy contributed to overall enjoyment in a direct fashion that was wholly unmediated by the concomitant feelings of being moved. Another study replicated the findings of Hanich and colleagues for sad music (Eerola et al. 2016). On a similar note, a recent study has shown that sad music is hedonically rewarding because it also and even predominantly evokes feelings of nostalgia and tenderness in Western listeners and feelings of peacefulness and tenderness in Eastern (Asian) listeners (Taruffi \& Koelsch 2014; see also Huron 2011; Kawakami \& Katahira 2015). Thus, it is not sadness qua sadness as a negative emotion that is liked and that contributes to aesthetic appreciation. Rather, what ends up being enjoyed are specific metonymical configurations of specific instances of sadness with a very circumscribed range of other emotional responses. Therefore, treating the topic of "sad" artworks in complete abstraction from the nearer and broader neighborhood of the respective sadness elicitors can easily be misleading.

In episodes of being sadly moved, a pity/compassion/ empathy factor (de Vignemont \& Singer 2006; Eerola et al. 2016; Keen 2006; Singer \& Lamm 2009; Singer et al. 2004) is often part of the emotional mélange (cf. Menninghaus et al. 2015b). Therefore, the enjoyment qua meta-emotional reappraisal of one's own feelings of empathy and the enjoyment qua being moved hypotheses do have some overlap. At the same time, the enjoyment qua being moved hypothesis is by no means limited to self-gratifying (meta)implications of feeling empathy for the plight of others. Sad films or narratives typically do not merely represent sad events; rather, they mostly feature friends, family, bystanders, and other witnesses who observe the plight of the protagonists already in the plot of the artwork itself, and at least some of these observers show prosocial responses of empathy and compassion and, occasionally, deep respect and admiration. Hence, more often than not, readers and viewers of sadly moving artworks can directly observe positive prosocial responses to sad events as depicted or displayed in the sadly moving artworks themselves and need not exclusively construe these in a meta-emotional fashion as a wholly self-reflective appraisal of their own empathic feelings. In other words, the enjoyment qua being moved hypothesis provides a broader cognitive basis for the positive feelings that sad films and poems have been shown to elicit, one that is not confined to a meta-appraisal of one's own feelings. At the same time, this hypothesis does not formally reject the meta-emotion hypothesis, but rather integrates it as one potential dimension within a more comprehensive framework.

Sadly moving real events, such as funerals, can similarly elicit positive feelings of high esteem, gratitude, and respect for the deceased person, along with a sense of social bonding among the survivors and, potentially, also of a self-rewarding beauty of the ceremony. Because being moved is mostly tied to a witness position (Tan 2009) and hence typically does not activate any attempt to change the respective emotion-eliciting event (Menninghaus et al. 2015b), emotional responses to real funerals experienced in person, on the one hand, and in response to art- and media-represented funerals, on the other, may in some cases not differ very much. The same, however, would not apply to experiencing real versus media-elicited horror, because in this case the appraisal of one's own safety being challenged would yield a strong affective difference for the two contextual framings. Hence, the example of real funerals does not challenge the importance of an art framing, but only shows that specific cognitive appraisals have a strong influence on how greatly emotional responses differ dependent on the activation of an art or ordinary reality framing. Presenting a filmic representation of a funeral as a documentary or as part of a fictional movie would enable investigation - however, within a shared representation framing - of the subtle differences in emotional responses that are still likely to derive from the ontological distinction between real represented and fictional represented events.

\subsubsection{The case of horror (fear, fearful dread)}

Research on horror films has provided evidence that increased levels of negative affect while watching horror films are associated with greater enjoyment (cf. Hoffner \& Cantor 1991; Sparks 1991; Zillmann et al. 1986; Zuckerman 1979) and, more specifically, that habitual horror film viewers positively embrace not just emotional antidotes, such as moments of relief and happy endings, but the fearful feelings themselves (Andrade \& Cohen 2007).

Given the design of the studies mentioned above, the self-reported positive affect is far from easy to interpret. It may have, at least partly, been a response to the actors, superb special effects, the setting, the editing, the plot construction, the soundtrack, and/or other dimensions of the artistic making of the films. However, nuanced measures of aesthetic appreciation were not made in these studies. Regarding emotional responses, data for positive and negative affect were routinely collected, and additional data for happiness and fear were collected in at least some of the studies (Andrade \& Cohen 2007). However, even though an important role of suspense and thrill seeking is widely acknowledged in the literature on horror films (Hoffner \& Levine 2005), we know of only one study (Sparks \& Ogles 1994) that has collected both suspense and enjoyment ratings in addition to those for fear. Only these three measures together - ideally complemented by measures for arousal, positive, and negative affect - would allow a mediation analysis testing the hypothesis that suspense-driven arousal is an important factor in the co-activation of positive and negative affect and that this factor may be instrumental in making fear/horror enjoyable. Pursuing other research questions, however, the aforementioned study did not perform such a mediation analysis and also not a correlation analysis.

Feelings of suspense have been shown to be pleasurably experienced in response to many plot-based artworks, be these literary narratives, dramas, or films, including horror films (for the latter genre, see Hoffner \& Levine 2005; Zillmann 1980; Zillmann \& Weaver 1996). Narrative suspense is a state of cognitive uncertainty regarding the outcome of a plot trajectory; it can go through varying degrees of fearful and hopeful anticipations, and it creates a need for resolution, which may or may not turn out to comply with the 
Menninghaus et al.: The Distancing-Embracing model of the enjoyment of negative emotions in art reception

reader's or onlooker's expectations and wishful desires (cf. Anz 1998; Berlyne 1960; Carroll 1996; Fill 2007; Hanich 2014; Lehne \& Koelsch 2015; Löker 1976; Zillmann 1980). Oscillating as they do between fearful and hopeful anticipations, states of suspense can be ranked among the states of mixed affective nature (Madrigal \& Bee 2005). (Because the pertinent discussion does not address the issue of negative emotions, we here do not discuss the question of whether or not suspense can be experienced in repeated exposure to the same literary or filmic narratives; see Carroll [1996], Gerrig [1997], Mag Uidhir [2011], Prieto-Pablos [1998] Smuts [2009b], and Yanal [1996].)

Zillmann's theory of suspense (1980) stipulates that it is the happy end that turns a suspenseful trajectory into a self-rewarding experience. Alternative theories, however, allow for positively appreciating the suspenseful trajectory itself. Specifically, the psychological construct of sensation seeking (Zuckerman 1979) suggests that the cognitive uncertainty and affective ambiguity between fear and hope can be experienced as inherently self-rewarding, if and to the extent that these negative cognitive and affective aspects of suspense fulfill needs for affective and physiological arousal that are different from a need for a happy resolution. Thus, fearful dread may feed and maximize the suspenseful emotional arousal that horror film viewers might ultimately seek and experience as self-rewarding, irrespective of what the end is like; after all, most typical recent media products of this genre lack a happy ending. Confirming this assumption, frequent viewers of horror films report that "the jolt of horror is exhilarating" and leaves them "feeling invigorated" (Tamborini \& Stiff 1987, p. 425); they expressly view horror films "as a way to get an adrenaline high or to feel pumped up and alive" (Robinson et al. 2014, p. 46). Andrade and Cohen's interpretation of their data (2007) is likewise compatible with the hypothesis that the enjoyment of fear/horror may be mediated through experiencing suspense.

If this hypothesis were to withstand further testing, it would follow that the arousing nature of horror films has two sources, fear/dread/horror and suspense-driven arousal, with suspense being of a mixed affective nature and thereby potentially facilitating - in conjunction with the art framing - the positive reevaluation of the negatively arousing nature of fear (horror). (On a qualifying note, this hypothesis may not apply to horror film viewers who appear to directly draw positive enjoyment from endorsing, if not identifying with, the aggressive and often sadistic behavior of the perpetrators [cf. Oliver \& Sanders 2004; Shaw 2001].)

Mixed emotional states of suspense are likely to contribute to enjoyment associated with negative emotions way beyond the particular genre of horror films. As already pointed out in Section 2, narratives and dramas, including comedies, routinely involve social conflicts, obstacles, suspenseful states between fear and hope, and the negative feelings associated with such conflicting predicaments (Grodal 2007; Krämer \& Witschel 2010; Scalise Sugiyama 2005). Moreover, if one considers musical tension - which has been shown to often involve interplays of positive and negative emotional cues on the levels of tempo, harmony, and other dimensions (Hunter et al. 2008) - as an analogue to narrative suspense, then the composition of temporal works of art, including literature, films, music, and dance, is routinely experienced as a temporal trajectory of suspense/tension and resolution/release (Huron 2006; Meyer 1961; Trehub 2000). Both the "sweet anticipation" of release (Huron 2006) and its actual experience in listening to music depend on a prior buildup of antagonistic tension-release patterns and on postponements of resolution rather than on an ongoing conformity to processing ease or pleasantness at no cost. Dissonances that increase partially unpleasant tension may well support stronger feelings of resolution and relaxation both during the online resolution of tension and afterward (Koelsch 2014).

Finally, to the extent that sad narratives and films also involve feelings of suspense, they are likely to recruit two mixed emotional states - being moved and suspense - for integrating negative feelings into overall pleasurable trajectories (for an example, see Hanich \& Menninghaus 2017). Thus, the mediator emotions we have discussed in separate subsections and with reference to two polar genres are by no means clear-cut alternatives, but can well be found in responses to the very same artworks and media products.

\subsubsection{The case of disgust}

Empirical evidence regarding the adoption of disgust for the pleasurable purposes of the arts is far less available than evidence regarding the enjoyment of sad films, poems, music, and horror films. Accordingly, this subsection is far shorter and of a more theoretical nature than the preceding two. Elaborate reflections by Nietzsche, Freud, Bataille, Sartre, Kristeva, and others (for a detailed account of this tradition see Menninghaus 2003, Ch. 5-9) converge in emphasizing that disgust has a potential to involve us in hidden, if not repressed, dimensions of profound pleasurability, or even jouissance. Freud's insistence that very young children like to play with their feces and even consider them as valuable gifts and that many feelings of disgust may be conceived of as repressed pleasure (cf. Menninghaus 2003, Ch. 6) is only the most prominent among the multiple, mostly philosophical theories that stipulate a partly positive reevaluation of the emotion of disgust specifically in art contexts. An empirical study focusing on the humorous and amusing implications of feelings of disgust in art contexts has provided nuanced evidence for a mixed affective nature of disgust in these contexts (Hemenover \& Schimmack 2007). Another recent study (Rozin et al. 2013) has likewise reported evidence for a reverse evaluation of potential elicitors and feelings of disgust. Thus, feelings of disgust in art reception may not only support hedonic processing by virtue of their sheer arousal value; the arts may also bring out (latent) pleasure dimensions (Korsmeyer 2011) that are typically not included in psychological accounts of disgust (Rozin \& Fallon 1987; Rozin \& Haidt 2013; Rozin et al. 2008; Tybur et al. 2013). Again, by no means do all otherwise disgusting feelings allow for such a positive reevaluation in art contexts; for example, to date, the genuine stench of corpses has never been incorporated into "disgust art," not even in Damien Hirst's provocative displays of decaying animal matter. Clearly, the arts implement a selective regime of compatibility and noncompatibility with enjoyment in the case of disgust, as well.

\subsubsection{Summary}

Summing up, this subsection argues - and partly provides empirical evidence - for the hypothesis that the arts tend 
Menninghaus et al.: The Distancing-Embracing model of the enjoyment of negative emotions in art reception

to draw on negative emotions in such a fashion that their elicitation ends up fueling and energizing neighboring or concomitant feelings of a mixed affective nature and that this metonymical contiguity with mixed emotions plays a mediating role for negative emotions' contributions to overall enjoyment. Supporting these assumptions from other vantage points, recent research in developmental psychology has shown that individuals are more motivated to explore, seek, and maintain negative affect if it is accompanied by positive affect and is hence part of a context that has a mixed affective nature (Riediger et al. 2009). Findings that complex mixed emotions play a stronger role in art reception than pure and simple negative emotions (cf. Krämer \& Witschel 2010; Oliver et al. 2009; Wirth et al. 2006) point in the same direction.

The hypothesis discussed throughout this subsection goes beyond the more abstract hypothesis of emotional antithesis discussed in the preceding subsection in that it adds an intermediate-mixed emotions - to the compositional interplay of positive and negative emotions. It attributes a crucial mediator role to this additional player and highlights the importance of particular contiguity relations among the interacting emotions in affect space, thereby substantially limiting the particular ranges, or instances, of individual negative emotions that are eligible for being adopted for pleasurable purposes. Because interplays of positive, negative, and mixed emotion are even more emotionally varied than those of positive and negative emotions only, they are likely to support the benefits of the latter-more emotional variety and dynamic changes - at least to an equal degree. In fact, one might well find upon closer inspection that complex emotional states of a mixed affective nature always play a role in the integration of the powers of negative emotions into altogether pleasurable trajectories. In that case, the compositional interplays discussed in sections 4.1 and 4.2 would not be categorically different anymore.

It is highly likely that other emotional states that are either routinely or frequently of a mixed emotional nature serve a role similar to the one we have diagnosed for being moved and suspense. The validity of this assumption could be tested on awe (Keltner \& Haidt 2003; Silvia et al. 2015) and feelings of the sublime (Eskine et al. 2012; Gordon et al. 2017), nostalgia (Wildschut et al. 2006), surprise (Noordewier \& Breugelmans 2013; Silvia 2009), and even special variants of confusion (Silvia 2009; 2010). If such tests yield positive results, one could even stipulate in a generalizing fashion that potential enjoyment of a particular negative emotion in art reception is dependent on the availability of a closely related mixed emotion that both is contiguous in affect space and can readily co-occur with the respective negative emotion. Speculative as though this consideration is, it emphasizes the importance of defining, as we did in this subsection, additional constraints that need to be met for a particular negative emotion to support intensely felt and memorable pleasurable experiences in art reception. For the time being, the range of negative emotions that meet the constraints defined above appears to be fairly limited.

\subsection{Aesthetic virtues of the artistic uses of the media of representation}

Following the system of poetics, the next step after treating the selection/invention and the combination of the major constituent parts of artworks is to consider the ways in which artworks use their specific media of representation for emotion-regulatory purposes. Regarding this dimension that is found in all artworks, we propose the following hypothesis: Aesthetically appealing uses of the media of representation (such as sound/music, words/language, color/ shape) have the power to make the processing of negative emotional content or associations more enjoyable while not reducing, let alone erasing, negative emotional responses.

Importantly, all (re)presentational media not only represent something, but also are something on their own. They have their own materiality and specific powers to signify, represent, allude to, or evoke some meaning and/or emotional response. The arts of writing, music, painting, and so forth are typically believed to make a special, more elaborate, and even partially alienating use of language, musical structures/musical performance, or color and shape compared with ordinary or more quotidian forms of language, singing, and painting (Dissanayake 2000). In fact, the artistic use of the very media of representation constitutes the material and phenomenal "reality" of artworks; it takes up a great portion of the artists' efforts, provides a reason for admiring their particular skills and performances (Newman \& Bloom 2012), and is likely to make a substantial contribution to aesthetic liking. Pleasure taken in the very art of representation is (primarily) not due to the processing of the object of representation, but rather to the aesthetic properties (Walton 1970) or virtues inherent to the representation itself (coloring, execution, poetic style, etc.; cf. Tinio \& Leder 2009; Tinio et al. 2011). Notably, all aesthetically appealing features of the wording used to represent, and reflect upon, a uxoricide in tragedy - Aristotle explicitly stressed the "sweetness" of diction, meter, and melody in the language and the sung portions of tragedy (1961, paras. $1449 \mathrm{~b}$ and $1450 \mathrm{~b}$ ) are by definition missing in the real event. As a result, the basis for an affective appraisal is likely to be different for a real event and for its artistic representation (cf. Friend 2007), because in the latter, the event represented interacts with the very means of artistically representing it.

Research on negative emotions in art reception has widely disregarded the aesthetic appeal of the purely formal quality of an artwork or media product. Studies on preferences for horror films are particularly weak in this regard: No other genre has prompted so many studies on the enjoyment associated with negative emotions, yet apparently none of these studies has experimentally modified the patterns of how these films employ lighting, coloring, camera perspective, techniques of cutting, special effects highlighting the monster's dreadful appearance, soundtrack, and so forth (for similar résumés, see the meta-analyses by Hoffner \& Levine 2005; Tamborini et al. 1990). Moreover, empirical evidence regarding the effects of special uses of the media of representation on the enjoyment of negative emotion is likewise scarce beyond the genre of horror films.

Positive statistical correlations between aesthetic liking, the intensity of being affected, and felt negative emotions have been reported in several studies on sad films (Bartsch \& Viehoff 2003; Bartsch et al. 2010; Hanich et al. 2014; Oliver 1993; Oliver \& Bartsch 2010; Wassiliwizky et al. 2015) and soundtracks of sad films (Eerola \& Vuoskoski 2011). Experimentally modifying 20 sadly and 20 joyfully moving poems that differ substantially in time of origin, a recent study (Menninghaus et al. 2017) investigated the effects that features of poetic parallelism as defined by Roman Jakobson (1960) exert 
Menninghaus et al.: The Distancing-Embracing model of the enjoyment of negative emotions in art reception

on emotional response dimensions (being moved, sadness, joy), on unipolar ratings of overall positive and negative affect, on several measures of aesthetic appreciation, and on the general impression of the intensity of being affected by the poems. The presence versus absence of the target patterns of poetic diction increased feelings of sadness, being moved, intensity, and positive affect (in the case of the sadly moving poems) and of joy, being moved, intensity, and positive affect (in the case of the joyfully moving poems). Importantly, ratings for sadness correlated positively with ratings for beauty, aesthetic liking, melodiousness, being moved, intensity, and positive affect. Hence, the artistic treatment of language was shown to enhance the positive aesthetic appreciation of poems of a primarily sad content, even without reducing, let alone converting, ratings for felt sadness and overall negative affect. This parallel increase in perceived feelings of sadness and being moved, perceived aesthetic virtues, and perceived overall intensity strongly supports the model presented here. Because parallelistic diction in general enhances ease of prosodic processing (Obermeier et al. 2016) - albeit often at the expense of making semantic processing more demanding (Menninghaus et al. 2015a) these findings also speak to an important contribution of processing fluency (Alter \& Oppenheimer 2008; Oppenheimer 2008; Reber 2016; Reber et al. 2004) to the enjoyment of negative emotions.

Analogous effects are likely to be achieved through the special artistic uses of the respective representational media in other art forms, as well. Supporting this assumption, a large share of music that is perceived as "sad" is also perceived as outstandingly beautiful; moreover, selfreports regarding responses to sad music suggest that individuals find it rewarding to imagine that, on occasion, they would have the same expressive power and potency as the sad musical pieces they like (Taruffi \& Koelsch 2014). This imaginative transfer, too, appears to be strongly dependent on the perceived artistic achievement of the pieces of music in question and, hence, clearly different from a mere effect of psychological distancing.

\subsection{Redeeming negative emotions through (symbolic) meaning making}

The construction of (symbolic) meaning is yet another level of art processing for which negative emotions are not just stumbling blocks, but also positive contributors. Here we propose the hypothesis: Interpretive efforts toward meaning making contribute to (re)appraising negative emotional content and concomitant feelings in a (more) positive and enjoyable light. The interpretation of ancient Greek tragedies is a classical case. For example, in line with the understanding of tragedy promoted by Hegel (1970, p. 547) and other philosophers, Bullough (1912, p. 104) suggested that "real tragedy ... truly appreciated, is not sad ... it is an homage to the great and exceptional in man."

The search for and discovery of some sort of meaning is a frequently used cognitive strategy for either retroactively or simultaneously (re)appraising negative events in a more favorable light (Giuliani \& Gross 2009; Gross 1998; Gross \& Thompson 2006; Larsen \& Prizmic 2008; Ochsner \& Gross 2005; Oliver et al. 2009; Oliver \& Woolley 2010). In recent media psychology, reevaluating negative affect from the perspective of a higher-order meaning(fulness) specifically plays a large role in studies on the enjoyment of sad films (Bartsch 2007; 2008; Bartsch \& Viehoff 2003; Bartsch et al. 2010; Oliver 1993; Oliver \& Bartsch 2010; Oliver \& Woolley 2010). Meaningfulness is what Fechner (1876, pp. 238-40) called a "reconciliatory moment." The heroic death for a good cause is a conspicuous-and often highly ideological-model for such a moment, one that is not even limited to the precondition of an art framing. However, in art contexts, the art framing is always additionally in place, and this should yield differences on some appraisal-driven dimensions of the emotional responses (cf. Menninghaus et al. 2015b).

Thus, contrary to Zillmann's hypothesis $(1971 ; 1991$; de Wied et al. 1994), plots that draw heavily on negative emotions by no means need to have happy endings to allow for markedly positive affective (re)appraisals. A prototypical happy ending, although clearly important for reevaluating negative affect in many cases, is only one of many ways to integrate negative emotions into an overall pleasurable trajectory by mixing them with positive ones. Notably, sensation seekers are far less likely to search for a higher-order level of symbolic meaning in horror films, and the same holds for consumers of disgust comedies. We therefore suggest that the meaning-construction route to accommodating negative emotions may only be an option for select cases.

\subsection{Genre scripts as emotion-regulation scripts}

Acquired genre schemata, or genre scripts, are different from the highly abstract schemata of art, representation, and fiction in that they entail fairly detailed anticipations as to which specific emotions/emotional tonalities recipients are likely to be going through and to what extent emotionally negative content is likely to elicit negative feelings in recipients. In other words, genre concepts entail dimensions of affective forecasting (Wilson \& Gilbert 2003; 2005) and hence serve to pre-adjust expected emotional responses (cf. Menninghaus 1999). Here we argue for the following hypothesis: Activation of particular genre scripts can contribute to (re)appraising negative emotions in a positive and enjoyable light.

In his essay The Uncanny, Freud (1955) noted that a narrative content feature that evokes strong feelings of the uncanny in a fantastic horror narrative is likely to elicit no such feelings when encountered in a fairy tale. In a similar vein, exposure to disgusting matters is likely to be perceived differently in a disgust comedy than in a tragedy (cf. the case of Philoctetes as discussed by Lessing 1766/1984). Anticipating recent experimental evidence from film studies (Visch \& Tan 2007; Visch \& Tan 2008; Visch et al. 2010), Freud contended that an artful writer commands high skills for manipulating the reader's mind frame by means of subtle stylistic cues rather than explicit instructions of a propositional kind (Freud 1955).

To date, genre attributions primed by subtle stylistic cues have not been empirically tested for effects on negative emotion processing. All existing studies have used explicit propositional framing instructions, and these framings have been used exclusively to contrast fictional literary texts with nonfictional news texts (Altmann et al. 2014; Zwaan 1994) rather than different literary genres with specific affective profiles. Freud's remark clearly calls for experimental testing by means of inserting text passages 
Menninghaus et al.: The Distancing-Embracing model of the enjoyment of negative emotions in art reception

of identical wording into different genre contexts that prime different meaning attributions.

\subsection{Summary}

This section spells out the five hypotheses that underlie the second pillar of our model (the Embracing factor; see Fig. 1). The majority of these hypotheses have previously not been part of the psychological theorizing about pleasure associated with negative emotions, at least not in a more elaborate form. Notably, our model does not rely on compensatory mechanisms (cf. Carroll 1990b), if compensation means that negative emotions first have a wholly negative effect on the enjoyment of art which is then healed by positive antidotes. After all, the top-down activation of art, representation, and fiction framings preemptively alters important appraisal dimensions of the negative emotions, and genre scripts have similar a priori effects on affective processing. Moreover, from the very beginning, the experiencing of negative emotions during art reception is inextricably linked to the aesthetically rewarding virtues of the artistic representation and to the interplay with positive and mixed emotions as described in this section. Therefore, we conceptualize our model not as a compensation model, but as a two-factor transformation model comprising the a priori Distancing of negative emotions (factor 1) and several mechanisms of Embracing the distanced negative emotions for pleasurable purposes (factor 2). Our model is also not a model of conversion, if conversion means a full-blown transformation of negative into positive affect. After all, we consider it necessary that negative emotions are actually experienced as such, at least within the constraints of the Distancing factor.

\section{Limitations and additional future directions}

As emphasized in the Introduction, our eight-component model is not a component-process model in any narrower meaning of this term. It hypothesizes that the identified processing components are relevant for negative emotion processing across art domains, yet leaves it to subsequent studies to test how readily these eight components can be integrated into process models of the individual arts.

Some components of the Embracing factor-most notably, the components "compositional interplays of positive and negative emotions," "aesthetic rewards of the very form of representation," and "emotion-regulatory implications of particular genre scripts" - have a substantial tradition in treatises on poetics and aesthetics. This raises the question whether artists employed the respective means of representation in a theory-guided fashion or based on intuitive knowledge only. Letters and other testimonies of artists could be scrutinized for evidence of conscious, theory-based anticipations regarding the emotion-regulatory effects of the respective strategies of representation.

Potential additional explanatory mechanisms likewise need to be considered. This applies specifically to the hypothesis that the co-occurrence of enjoyment and negative emotions when viewing horror films and similar media products might be explained as a benign variety of genuine masochism, that is, of the physical and psychological pain that masochists embrace as (sexually) pleasurable (Bloom 2011, pp. 51-52, 194-97). Rozin et al. (2013) have surveyed a broad variety of activities that may be accounted for with the help of this theoretical explanation; art-specific processing mechanisms were not considered in this context. Future studies will therefore need to investigate whether the benign-masochism hypothesis can indeed explain in a very parsimonious fashion all effects that we here ascribe to several art-specific processing components.

For comparative reasons, it would also be interestingeven though very difficult for both reasons of ethics and study design - to investigate non-art instances that eighteenth-century treatises on aesthetics routinely discussed in the context of the topic: the notorious attraction of gladiator's fights and public executions and also of apocalyptic visions and catastrophes.

Finally, the powers of negative emotions to secure attention, intense involvement, and privileged access to memory are likewise recruited - albeit not within the activation of an art framing - by news reports, political speeches and propaganda, and commercial ads. Considering that classical rhetoric and poetics essentially used the very same framework for analyzing poetic, political, and other pragmatic speech, our model may serve as a basis for comparing the role of negative emotions across these different domains.

\section{Conclusion}

Revisiting a long tradition of rhetoric, poetics, and aesthetics in the light of recent empirical and theoretical work in both the psychology of emotions and aesthetics, we propose a novel integrative account of the aesthetic pleasure/enjoyment associated with negative emotions. Our DistancingEmbracing model (Fig. 1) features two groups of processing components. The first keep negative emotions at a cognitive appraisal-driven distance, thereby preventing them from being outright incompatible with the hedonic expectations of art reception. This sets the stage, or clears the ground, for the second group of components. The latter even positively adopt, or embrace, the particular powers of negative emotions in the service of intensifying overall enjoyment and rendering the trajectory of art reception more varied, interesting, and profound in its affective nature and less prone to induce boredom. In the light of this model, negative emotions are not a special, let alone paradoxical, license for particular art forms only. Rather, their powers are an important, valuable resource for the arts and art reception in general, and our model spells out the mechanisms that allow the hedonic bias of art reception to thrive precisely on the negativity bias (Cacioppo et al. 1999; Ito et al. 1998; Rozin \& Royzman 2001) of our emotional system.

\section{ACKNOWLEDGMENTS}

We thank Michael Eid, Klaus Scherer, and John T. Cacioppo for their comments on an earlier draft of this article, and Rolf Reber and four anonymous reviewers for their many valuable critical remarks and suggestions for alterations. We also thank Philip Ekardt, Daniela Schönle, and Mira Shah for their support in the broad research efforts that made this article possible.

\section{NOTE}

1. Correspondence concerning this article should be addressed to Winfried Menninghaus, Max Planck Institute for Empirical Aesthetics, Grüneburgweg 14, 60322 Frankfurt am Main, Germany. E-mail: w.m@aesthetics.mpg.de. 


\section{Open Peer Commentary}

\section{Positivity versus negativity is a matter of timing}

\author{
doi:10.1017/S0140525X17001571, e348
}

\author{
George Ainslie ${ }^{1}$ \\ School of Economics, University of Cape Town, Rondebosch 7710, \\ South Africa. \\ Department of Veterans Affairs, Coatesville, PA 19320. \\ George.Ainslie@va.gov \\ www.picoeconomics.org
}

Abstract: "Negative" emotions are never purely negative. They attract attention at the very least and often stay attractive enough to make rehearsing them an addictive activity. As the authors point out, they also counteract a relentless tendency for positive emotions to become boring. Analysis in terms of reward suggests why this tendency occurs and how symbiosis with negative emotions may arise, in art and in life.

Menninghaus et al. ask why negative emotions are "central in art reception far beyond tragedy" (abstract). They catalog eight mechanisms by which painful affect increases pleasure, but their hypotheses rest basically on two: making the pain not so bad (art context, mere representation, and fiction), which does not speak to the change of valence, and mixing pain and pleasure, the positive effect of which is itself what they promised to explain (sect. 1). The other "embracing" phenomena-aesthetic virtues, meaning construction, and genre scripts-are beside their main quest for what turns the negative into the positive.

This quest should reach well beyond the realm of art. Why are negative emotions central to the pursuit of pleasure generally? To the extent that the world does not impose painful feelings on us, why are we moved to seek them? The authors' core hypothesis about "aesthetic processing" is that "the pleasure taken in the beautiful representation of wholly positive and beautiful objects and narratives tends to be less intense, profound, and self-supportive and more prone to induce boredom than pleasure that includes a dynamic interplay of positive and negative emotional responses" (sect. 4.1, para. 1). As in art, so in life. People often seek the adjuvant effect of negativity, for instance, in gambling despite a conscious expectation of loss, masochistic relationships, painful sex, and endurance sports. Many apparent pains maintain behaviors over long periods, such as prolonged grieving, nursing grudges, and following disaster-oriented news sources. And these are just cases in which a distinct negative component makes the question of rationality obvious.

In many more ways people court obstacles, defeats, and challenges while concealing from themselves that they are doing so (Ainslie 2013). Conventional utility theory requires that we set long-term goals and defend them from risky, "impulsive" distractions, but we have been warned that such policies may disappoint us. Emotion researcher Sylvan Tomkins noted: "The paradox is that it is just those achievements which are most solid, which work best, and which continue to work that excite and reward us least. The price of skill is the loss of the experience of value - and of the zest for living" (1978, p. 212). Ethologist Konrad Lorenz described the result of living in leisure: "The whole glorious amplitude of the waves of human emotions, all that makes life worth living-is dampened down to a scarcely perceptible oscillation between scarcely perceptible tiny displeasures and pleasures. The result is an immeasurable boredom" (1970, pp. 355-56). A prosperous society has to choose involvements the way it chooses art, finding "a dynamic interplay between positive and negative emotional responses."

So the negative can be an investment in refreshing the positive, but what properties does this imply? I propose a reward-based analysis to reconcile the sometime positive effect of negative emotions with utility theory. Like the authors, I put aside "functional benefits" (sect. 1, point C) and deal entirely with differential motivation.

1. Reward is whatever makes the processes that precede it more likely to be chosen again. This definition is broader than the commonsense notions of pleasure or satisfaction, because addictive rewards or the rewards of an itch may provide pleasure without satisfaction, and gratifying an urge to panic provides neither.

2. Emotions are reward patterns that probably evolved to give momentum to one or another behavioral tendency in particular circumstances; but however specific their triggers may be, they work through reward. Emotions' power to pre-empt attention implies that they generate quick reward. In "negative" emotions, this reward lures us into participation, but subsequently blocks richer sources of reward to a greater or lesser extent: Grief interferes with new attachments, disgust with appetite, anger with openness, fear with any activity requiring relaxation. Unless these emotions are linked to potential longer-term positives, they do not repay the attention they demand; so we try to escape them.

3. Emotional patterns compete based on the time courses of their rewards. When we have poor prospects for better alternatives, or where our susceptibility to the negative emotion is great, emotions that most people find frankly negative may lure us repeatedly: hence pathological grief, phobic anxiety, and paranoid personality disorder. The case of anger involves a closer balance-occasions for it are often sought robustly, are cultivated habitually in hatreds, and are negative only to the extent that the anger spoils richer relationships (Lerner \& Tiedens 2006). Such emotional patterns, which engage many people despite rewarding poorly over time, are catalogued in medieval Christianity's seven deadly sins: not only wroth, but also envy, pride, avarice, sloth, lust, and gluttony. Grief and fear were apparently too aversive to be sins, although depression was sometimes counted as a form of sloth (Irvine 1999).

4. Positive emotions soon fatigue, at least in part because our innate preference for smaller, sooner reward over larger, later reward leads us to harvest their rewarding potential too early, as in daydreaming (modeled in Ainslie 2005; 2017). ${ }^{2}$ The attenuation of positive emotion leads to the "scarcely perceptible oscillations" that Lorenz complained of, and creates an incentive to accept emotions that would simply be negative except for their property of refreshing the pleasurable emotions. At the very least, a negative emotion obstructs our greed for quick satisfactions, letting the potential build. As the authors suggest, some forms of refreshment are more complex than that (sect. 2).

5. It is hard to model how relatively negative emotions get a bonus from building the readiness for positive ones. The relationships of emotions are opaque, even with respect to what emotions can be discerned to satiate or fatigue independently from each other (in the way that physical appetites can be [Herrnstein 1969]). The authors raise the interesting possibility of studying the "family relationships" of emotions in "affective space" by observing the occurrence of "metonymical transfer" (sect. 4.2). A study of how an emotion can "inform and partly transform co-occurrent" (sect. 4.2, para. 2) neighbors would be phenomenological, but not necessarily unrelatable to reward-based analysis. The arts are a rich source of recognizable examples, as used for instance by Elster (1989).

6. We choose art that has an admixture of negative emotions if we expect a net positive effect that is great enough - again, disproportionately discounted for delay. The greatest satisfactions come after the hardest experiences; in the authors' words, "higher levels of negative emotions ... support more intense emotional responses" (sect. 2, para. 6). Thus, one task of art is to invite acceptance of greater negativity during the work. This will be a collaborative task with the audience, who must let the hedonic importance of their involvement build over periods of threat and deprivation (Ainslie 2017). If you distance yourself when a negative emotion is intense say to yourself "this is only a story" - it will reduce your payoff and likely reduce your ability not to say it when you encounter negativity again. Awareness of such a recursive effect increases our commitment to accept negativity, in art as in life. 


\section{NOTES}

1. This material is the result of work supported with resources and the use of facilities at the Department of Veterans Affairs Medical Center, Coatesville, Pennsylvania, and is thus not subject to copyright in the United States. The opinions expressed are not those of the Department of Veterans Affairs or of the U.S. Government.

2. People in whom this does not happen as much become absorbed in imagination - are "fantasy prone" (Rhue \& Lynn 1987).

\section{Considering the filmmaker: Intensified continuity, narrative structure, and the Distancing-Embracing model}

\author{
doi:10.1017/S0140525X17001583, e349
}

\section{Kacie L. Armstrong and James E. Cutting \\ Department of Psychology, Cornell University, Ithaca, NY 14853-7601. \\ kla78@cornell.edu james.cutting@cornell.edu \\ https://www.researchgate.net/profile/Kacie_Armstrong \\ http://people.psych.cornell.edu/ jec7/}

\begin{abstract}
Menninghaus et al. pose two open-ended questions: To what extent do formal elements of art elicit negative affect, and do artists try to elicit this response in a theory-based or intuitive manner? For popular movies, we argue that the consideration of their construction is prior to the consideration of the experience that they evoke.
\end{abstract}

Filmmakers rely on the ability of audiences to suspend disbelief and embrace the stories told on screen. However, the degree to which a viewer embraces a cinematic narrative, including both the positive and negative emotions associated with it, may depend more on the filmmaker than the observer. Here, we discuss filmmaker tactics in relation to the Embracing component of the model proposed by Menninghaus et al.

By the incremental adoption of well-orchestrated, seamless editing strategies that result in narrative immersion, filmmakers have cultivated an art that, in a sense, demands to be embraced. Indeed, David Bordwell (2002; 2006) has described the result of such enhanced editing techniques as intensified continuity, that is, the use of rapid editing, increased close-up shots, and removal of extraneous narrative details to achieve a sense of narrational cohesion between shots and to render cuts less detectable by the average viewer. The details of these practices can be genre specific. For instance, action films, as compared with films of other genres, tend to feature shots that show fewer characters (Cutting 2015) and that are shorter in duration (Cutting et al. 2010; 2011), tighter in scale (Cutting et al. 2012), and higher in dynamic activity - car chases, gunfights, and physical altercations.

The implications of these stylistic conventions are twofold: they promote narrative transportation and they elicit overall positive affect in the viewer. In support of the former point, Bezdek et al. (2015) demonstrated that an increase in narrative suspense results in reduced activity in brain regions involved in peripheral visual processing, while brain activity associated with central visual processing and attention increases. In other words, the suspense experienced while viewing an action film, as driven by intensified continuity on the filmmaker's part, results in greater visual and cognitive processing of the fiction presented on screen, while reality beyond the screen goes unnoticed by viewers. Furthermore, once embraced, the rapid activity of films is linked to dopamine release (Grodal 2009), which is associated with reward and positive affect. This process supports the notion that the negative emotions potentially elicited by an action film (e.g., fright, nervousness, or surprise) are tempered by a subjective positive experience of the film overall.

It may seem counterintuitive that a film rife with car chases, hand-to-hand combat, thundering hooves, or explosions would elicit positive affect. To resolve this dissonance, we expand upon the fifth component of the Embracing factor of the Distancing-
Embracing model: the "emotion-regulatory power of specific acquired genre scripts" (sect. 5). The tendency for filmmakers to utilize low-level elements of film (e.g., shot duration, shot scale, motion, color, and luminance) in a formulaic manner speaks to particular aspects of appraisal theories of emotion.

Specifically, the notion that affect depends largely on the degree to which the outcome of an event matches one's expectations, rather than mapping directly onto the valence of the eliciting stimulus itself (Scherer 2001) may illuminate why audiences are continually drawn to films that reliably provoke negative affect. A gunfight is generally not considered to be a joyous occasion; however, if a filmmaker has made clear through both narrative content and subtle editing cues that the film being viewed is an action film, the viewer taps into certain expectations of how the film should proceed, given learned conventions of the genre. When such expectations are satisfied, the viewer's emotional response should depend not upon the affective valence of the gunfight itself, but rather the fact that the gunfight has happened at all (and, importantly, that the gunfight was portrayed through established manipulation of shot duration, shot scale, and motion).

Although viewer expectation is a product of the viewer's mind, whether such expectations are met rests in the control of filmmakers. In fact, recent evidence suggests that filmmakers may drive the satisfaction of audience expectation by visually amplifying the structure of established narrative trajectories. For instance, the darkest moment in a narrative, defined by Keating (2011) as the protagonist's emotionally lowest point occurring at about three-quarters of the way through a film, tends to be the visually darkest moment on screen (Cutting 2016). This finding suggests that filmmakers carefully construct a visual mood that encourages viewers to experience the negative emotions that are prescribed by narrative formulas, thus corroborating the viewer's learned expectations and, consequently, infusing negative emotions with positive affect.

Finally, consider the nature of filmmaking: Is it a theory-based art form or one that is implicitly executed? Bordwell (2002) describes the practice of continuity editing as having taken decades to cultivate, likely through cultural transmission across generations of filmmakers (Cutting \& Candan 2013; Cutting et al. 2010). In other words, through trial and error since the conception of film, filmmakers have learned to expertly accommodate human perceptual and cognitive mechanisms through the manipulation of low-level cinematic features (even without explicitly grasping the psychology behind their practice). Certainly, if one were to ask a filmmaker if she intentionally times shot duration to promote positive affect in her viewers, she would likely deny the role of any conscious knowledge of perceptual psychology in the success of her craft.

Perhaps, then, filmmakers appeal to affective experience via manipulation of the formal elements of film. They tacitly and intuitively build a visual foundation that fosters a clever interplay between the positive and negative emotions that are essential to the enjoyment of art. In effect, the embracing of artwork and its resulting negative emotions is not primarily the work of the viewer, but, rather, is the domain of the artist, who adheres to established artistic practices to carefully hone her work in service of a fundamental goal: to move her viewers.

\section{Art reception as an interoceptive embodied predictive experience}

doi:10.1017/S0140525X17001856, e350

\author{
Ruben T. Azevedo ${ }^{a, b}$ and Manos Tsakiris ${ }^{a, b}$ \\ ${ }^{a}$ The Warburg Institute, School of Advanced Study, University of London, \\ London WC1H OAB, United Kingdom; 'Laboratory of Action and Body, Royal \\ Holloway University of London, Egham TW2O OEX, United Kingdom. \\ ruben.azevedo@rhul.ac.uk manos.tsakiris@rhul.ac.uk \\ https://warburg.sas.ac.uk/about/people/ruben-azevedo \\ http://warburg.sas.ac.uk/about/people/manos-tsakiris
}


Abstract: In the Distancing-Embracing model, an explanation is proposed for the apparent paradox that is the enjoyment of negative emotional states in art reception. Here, we argue for the advantages of grounding the psychological dynamics described in the model in established and empirically testable frameworks of brain functioning by thinking of art reception as an embodied experience guided by predictive coding.

With the Distancing-Embracing model, Menninghaus et al. propose a compelling account of the psychological dynamics associated with self-sought hedonic exposure to negative emotions in art reception. However, we suggest that the model could benefit from the integration with current neurocognitive frameworks of brain functioning, namely, embodied cognition accounts and predictive processing theories.

Prevalent embodied cognition accounts argue that we perceive the world through our bodies. Not only is the awareness of oneself as being "here" and "now" grounded on the perception of our own body, but also emotional experiences are tightly connected to the dynamic representation of the body in the brain. Interoceptive signals arising from the inner body are known to intensify emotional experience (Critchley \& Harrison 2013) and bias perception and behavior (Azevedo et al. 2017), even when we are not aware of it. The ability to resonate and engage with others' actions and emotions is also connected to body representations. Converging evidence shows that when perceiving an action or emotion, either positive or negative, the observer uses his or her own body and neural architecture responsible for first-person experience to simulate and represent that of the target (Gallese \& Sinigaglia 2011). Regarding art reception, recent research has shown that the appreciation of artwork, such as a painting or dance, is partially grounded in the embodied simulation of the actions and emotions represented, as revealed by activity in sensorimotor cortices, limbic, and reward regions (Blood \& Zatorre 2001; Calvo-Merino et al. 2005; Freedberg \& Gallese 2007).

Importantly, the embodiment of others' emotions does not constitute the entire vicarious experience, but rather a fast and powerful mechanism to pre-reflectively resonate and engage with others. Indeed, numerous factors such as perceived similarity with the target, the context and motivations modulate vicarious emotional sharing and may even lead to the experience of emotions antagonistic to those of the target, as the experience of pleasure at the perception of other's pain (Cikara et al. 2011). In art reception, such modulating factors are evident in different patterns of brain and physiological responses observed across experts and non-experts (Christensen et al. 2016; Cross et al. 2011; Kirk et al. 2009a) and, more generally, in the fundamental distancing mechanisms that allow experiencing emotions through the lenses of art schemata. Thus, considering the body as a vehicle for art reception may help one understand the neurophysiological mechanisms underlying the experience of emotion in art and ground the psychological dynamics described in the Distancing-Embracing model in an established and testable framework. Moreover, studying art reception through the lens of embodied experience may also inform and advance the understanding of motivated vicarious experience of other's emotions in real-life situations, particularly in instances in which observers distance themselves from the target and/or experience incongruent emotions.

Predictive processing theories invert the classical conceptualisation of the brain as a passive bottom-up processor of sensory information. Instead, they propose that the brain is a hierarchical inference machine, constantly attempting to predict its inputs from the environment (Clark 2013). Incompatibilities between the brain's predictions and incoming sensory data give rise to "prediction errors" that need to be minimised by, for example, updating the brain's generative models (or predictions) or balancing the weight given to sensory information. In art reception, the ambiguities and violation of expectancies that characterise most artwork - whether they are violations of canonical forms and shapes in visual arts; variation in timing, intensity, or timbre in music; or chills in a movie-generate prediction errors that need to be minimized. Recent theoretical proposals (e.g., Salimpoor et al. 2015; Van de Cruys \& Wagemans 2011) and initial empirical evidence (e.g. Salimpoor et al. 2013) propose that art production and appreciation lie precisely on the generation and resolution of such prediction errors. Not only predictive errors and their minimisation are rewarding per se, as evidenced by the release of dopamine and activation of brain reward systems (e.g., Salimpoor et al. 2015), but also art recipients take pleasure in the resolution of the perceptual/cognitive ambiguities and challenges posed by art.

Crucially, prediction errors are not exclusive to the exteroceptive domain - vision, hearing, touch - but also emerge from interoceptive sensations (Barrett et al. 2016). Changes in physiological states, such as heart rate and respiratory rhythm, caused by, for example, the perception of a moment of intense passion or acute fear in a film, give rise to interoceptive prediction errors that inform the brain of substantial unexpected physiological changes that need to be dealt with to restore homeostatic equilibrium. If the mismatch between predictions and interoceptive prediction errors is significant enough, the changes in the interoceptive body will come to the individual's awareness who may, for example, engage in emotion regulation processes to reduce physiological arousal or reinforce the art-related Distancing cognitive schemes that allow a "safe" and pleasurable experience of intense emotional states. Importantly, given the fundamental role of the brain in homeostatic regulation, these prediction errors have important motivational relevance and constitute the basis for the subjective experience of emotions (Barrett et al. 2016).

We propose that the experience of emotion in art reception relies to a great extent on the continuous updating of predictive models of ongoing (interoceptive) bodily states as we respond to the prediction errors that the artwork imposes on us. Most of the phenomena described in the model's components-compositional interplays of positive and negative emotions and mixed emotions as mediators of negative emotions - are indeed particularly powerful instances of violation of expectations, with strong motivational content and substantial changes in the representation of physiological states. Ambivalent emotions, the description of an act of sacrifice in a novel, the sudden happy twist in a film, or the building up and release of tension in a horror movie or musical piece are all likely to induce substantial interoceptive prediction errors that need to be explained away, rendering art reception a rich embodied experience. It is this embodied response to art that explains how it moves us in ways that cannot be easily explained by considering only the beholders' exteroceptive perceptual system and their cognition.

ACKNOWLEDGMENTS

M.T. and R.T.A. were supported by the NOMIS Foundation Distinguished Scientist Award to M.T. for the project Body \& Image in Arts \& Science (BIAS).

\section{Emotional granularity and the musical enjoyment of sadness itself}

\section{doi:10.1017/S0140525X17001595, e351}

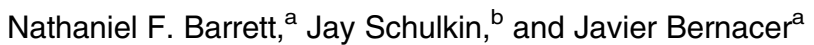 alnstitute for Culture and Society, University of Navarra, Pamplona 31009, Spain; ${ }^{\mathrm{b}}$ Department of Neuroscience, Georgetown University, Washington, DC 20057. \\ nbarrett@unav.es jjs54@georgetown.edu jbernacer@unav.es http://www.unav.edu/web/instituto-cultura-y-sociedad/mente-y-cerebro http://neuro.georgetown.edu/}

Abstract: We contest the claim that musically induced sadness cannot be enjoyable in itself. This possibility is supported by closer attention to a musical experience as well as cases of affective reversal, such as the 
"hedonic flip" of painful feelings. We propose that the affective reversal of sadness in music is due to the high granularity of musically induced emotion.

We commend the authors of the target article for their comprehensive analysis of the many factors that contribute to the aesthetic enjoyment of negative emotion. However, without discounting the importance of these factors, we wish to contend - particularly in the case of musical emotion - the authors' claim that "it is not sadness qua sadness as a negative emotion that is liked and that contributes to aesthetic appreciation" (sect. 4.2.1, para. 6) (for a similar claim see Juslin 2013, p. 258; cf. Vuoskoski \& Eerola 2017). According to their Distancing-Embracing model, it is not the negative emotions themselves that are enjoyed, but rather the way in which they combine with other emotions so as to enhance the overall aesthetic experience. But in many cases of musical enjoyment, we believe that sadness itself is enjoyed. Here we provide reasons for considering musically induced sadness as enjoyable in itself and suggest ways in which current and future research in neuroscience and psychology might help shed light on this possibility.

First, the notion that "distancing" is required for the aesthetic "embrace" of an otherwise unpleasant negative emotion does not fit well with experiences of negative emotions in music. On the contrary, listeners and performers of music seem to enjoy negative emotions precisely to the extent that they are powerfully affected by these feelings. The pleasure of musical immersion in negative emotion is supported by numerous accounts of strong musical experiences (Gabrielsson 2011), as well as a recent study that indicates that the enjoyment of sadness in music is strongly linked to "being moved" by sadness (Vuoskoski \& Eerola 2017). Furthermore, there is no evidence from selfreport studies that subjects find musically induced feelings of sadness to be unpleasant in themselves and, thus, in need of "distancing” (e.g. Taruffi \& Koelsch 2014; Vuoskoski et al. 2012).

The idea that musical sadness can be enjoyable in itself is also the most parsimonious approach to the "paradox" of enjoyable sadness, as it targets the feeling itself and does not require that sadness is combined with other emotions or that it functions to restore "homeostatic balance" (Sachs et al. 2015). Why, then, is this idea rejected by psychologists of music (e.g., Juslin 2013)? The most likely reason is that the idea of enjoyable sadness seems oxymoronic, given that sadness is essentially negative. But this assumption can be questioned on both phenomenological and scientific grounds.

From a phenomenological perspective, the difficulty of pinpointing the "negativity" of negative emotions like sadness suggests that it is complexly constructed and cannot be reduced to a single factor. In particular, it seems that whatever felt qualities support the categorical discrimination of an emotion (e.g., as sad, happy), these qualities are separable from the affective tone that normally accompanies and is typically associated with this emotion. This is the upshot of the philosophical literature on affect, which finds that the specifically affective component of pleasurable and painful feelings is not marked by any essential phenomenal quality (e.g., see Aydede 2014). Likewise, from a scientific perspective, although we do not have a widely accepted neurocognitive model of affect (see Lindquist et al. 2016 for a review), evidence from a variety of sources indicates that negative affective tone is a variable ingredient in feelings that we commonly categorize as negative. The most striking example of this affective variability is pain. Regardless of how feelings of pain are typically experienced, any attempt to define pain as essentially negative is confronted by a wide variety of cases in which its negative affect is attenuated, absent, or even reversed (Glucklich 2001; Grahek 2007). For example, a recent study demonstrated that, depending on context, moderate pain could be experienced as positive, a phenomenon the authors term "hedonic flip" (Leknes et al. 2013). Affective reversibility might be explained by findings that pain and pleasure are controlled by the same brain areas-the nucleus accumbens, globus pallidus, and amygdala - and thus might belong to a common affective currency that can be modulated independently of other components of emotional experience (Leknes \& Tracey 2008). Interestingly, these same brain areas have been related to the pleasure of listening to sad music (Sachs et al. 2015). Again, we do not yet have a solid neurocognitive model of affect, but the upshot of pain research seems to be that feelings of pain are complex and context sensitive and, moreover, that the feeling of pain itself can change in respect of affective tone. Should not we expect the same of sadness?

Even if we accept that sadness can be transmuted by music into something enjoyable, this process is still in need of explanation. Drawing from recent research on the "complexity" or "granularity" of emotion (Barrett et al. 2001, Kashdan et al. 2015; Lee et al. 2017; Lindquist \& Barrett 2008; Smidt \& Suvak 2015), we have recently proposed that the enjoyment of sadness itself in music could be explained if it could be shown that musically induced emotions are more finely differentiated than normally induced emotions (see Barrett \& Schulkin, under review). Felix Mendelssohn famously observed that our experience of emotion in music is "too precise for words." This statement suggests that emotions are not just triggered by music, but rendered in highly differentiated form. Meanwhile, research on emotional granularity indicates that the fine differentiation of negative emotions alters their negative affect (Kashdan et al. 2015), although we are the first to suggest that high granularity can cause affective reversal. In any case, the role of emotional granularity in musical experience calls for further investigation: to our knowledge, no empirical study of musical enjoyment has focused on emotional granularity. The challenge is to find ways of comparing the granularity of musically induced versus normally induced sadness in the same individual, either through self-reports or through direct measures of neural activity (e.g., electroencephalogram). So far, no methods for the direct measurement of emotional granularity exist, but see Lee et al. (2017) for an exploratory study of the effects of emotional granularity on emotional processing.

\section{A social dimension to enjoyment of negative emotion in art reception}

\author{
doi:10.1017/S0140525X17001601, e352
}

\section{Brock Bastian}

Melbourne School of Psychological Sciences, The University of Melbourne, Melbourne, Victoria 3010, Australia.

brock.bastian@unimelb.edu.au

www.brockbastian.com

Abstract: The proposed model overlooks the contribution of a relational/ prosocial dimension to the enjoyment of negative emotion in art reception. Negative experiences have a unique capacity to build social bonds and may also increase motivation to "connect" with the artist. This affiliative motivation ensures that people experience an artwork as more emotional, more intense, more interesting, and ultimately more rewarding.

The target article by Menninghaus et al. provides a novel and intuitively compelling account of why people seek out and enjoy art which elicits negative emotions in the perceiver. This seemingly counter-hedonic behavior (with pro-hedonic consequences) certainly deserves a theoretical explanation. In this commentary, I propose an additional dimension to the Distancing-Embracing model which is not considered by the authors, specifically, that there is a relational/prosocial dimension to the enjoyment of negative emotion in art reception.

There is now an emerging body of work illustrating that negative emotional experiences provide a potent trigger for people 
to become more relational/prosocial in their orientation toward others. Anthropologists have long noted that many cultures around the world ritualize painful practices, suggesting that they do so to promote social cohesion (Durkheim 1912/1995; Whitehouse 1996). Incidental evidence for this effect of shared negativity is also evident in accounts of soldiers becoming bonded together through the trauma of war (Whitehouse et al. 2014) or increased prosocial behavior in response to catastrophic events (Penner et al. 2005).

More direct evidence is emerging. For instance, Xygalatas et al. (2013) found that participants in the kavadi, practiced during the Thaipusam festival in Mauritius, engaged in more prosocial behavior when they had endured greater levels of pain. Building on this work, Bastian et al. (2014a) found that even mildly painful experiences (e.g., cold-presser task, leg squats, chili consumption), when shared within a novel group, fostered perceptions of bonding and trust within the group and increased cooperative decision making in an economic game. A similar increase in prosocial behavior (e.g., trust and cooperation) was observed when male participants were exposed to social stress (von Dawans et al. 2012).

The evidence indicates that negative experiences can lead to an increase in affiliative behavior towards others, perhaps especially when these are shared (see also Bastian et al. 2014b). Although this work suggests a functional account - tending and befriending others under conditions of stress is an effective way of coping (Taylor et al. 2000) - it also highlights a source of enjoyment. People like to feel connected to others, and sharing the experience of negative emotion arising from art may also offer an avenue through which people can build positive and enjoyable social bonds.

Importantly, however, sharing negative emotional responses to art may go further in producing increased pleasure/enjoyment. Evidence suggests that sharing an experience with others amplifies both the pleasantness and unpleasantness of that experience (Boothby et al. 2014). Negative emotions in art, therefore, may not only directly increase the intensity of the experience through capturing attentional resources, but also through building social bonds with others, and this social bonding in turn increases the intensity of the art experience itself.

This social dimension of negative emotions in art may be apparent even when other observers are not present. Negative emotional responses to art may also increase a sense of connectedness that people feel to the artist themselves. Immersing oneself in an artist's impression of the world involves engaging with the artist's intentions and perspectives. Negative emotions should, therefore, increase feelings of affiliation towards the artist and a desire to "connect" with their motivations and perceptions.

The relational/prosocial dimension fits within the structure of the Distancing-Embracing model proposed by the authors. Feeling that a particular negative emotional experience is shared would act as a distancing factor, whereby the social nature of the encounter provides a buffer for negative and emotionally challenging content. Indeed, there is an abundance of evidence to show that social support buffers the impact of negative events such as physical pain (e.g., Brown et al. 2003). Increased affiliation in response to negative emotion in art should reinforce a sense of personal safety, especially when social resources are immediately available (e.g., watching a thriller with friends). This, in turn, should facilitate distancing.

In addition to distancing effects, the relational/prosocial dimension also highlights avenues through which people may embrace art. As reviewed above, affiliative motives which arise in response to negative experiences are likely to contribute to a sense that an experience is being shared, and in turn, this amplifies that experience. Furthermore, because negative emotions also increase a motivation to "connect" with (or embrace) the artist themselves, the artwork is likely to become more significant to the perceiver. Together, these two affiliative motivations, which are triggered by negative emotion, serve to make the reception of an artwork more emotional, more intense, more interesting, and ultimately more rewarding.

Although beyond the scope of the Distancing-Embracing model, a relational/prosocial dimension also suggests that other antecedent social factors such as extroversion and attachment styles may moderate the enjoyment that people derive from negative emotions in art. Furthermore, the current analysis suggests that functional benefits associated with negative emotion in art may extend beyond well-being effects (referred to in the target article) to social effects, suggesting that as with rituals, negative emotions in art reception may play a role in promoting social cohesion.

\section{The urge to judge: Why the judgmental attitude has anything to do with the aesthetic enjoyment of negative emotions}

\author{
doi:10.1017/S0140525X17001613, e353
}

\section{Elvira Brattico and Peter Vuust}

Center for Music in the Brain (MIB), Department of Clinical Medicine, Aarhus University, The Royal Academy of Music Aarhus/Aalborg, 8000 Aarhus C, Denmark

elvira.brattico@clin.au.dk pv@musikkons.dk musicinthebrain.au.dk

\begin{abstract}
Based on arguments from both philosophical and empirical aesthetics, we hereby propose that the enjoyment of negative emotions in art and fiction is distinct from the immediate pleasure deriving from sensory features, because it requires a conscious, intentional attitude toward the object. This attitude is linked with the compelling goal of providing a judgment of liking, beauty, perfection, or similar.
\end{abstract}

The model proposed by Menninghaus et al. is well constructed and comprehensive toward explaining the paradoxical phenomenon of enjoyment of negative emotions in art. They put forward two processing factors, each including several components. The Distancing factor, consisting of the cognitive schemata of art, representation, and fiction, modifies the appraisal of negative emotions, keeping them at some "psychological distance." The Embracing factor, consisting of the interplays of positive and negative feelings, mixed emotions, aesthetic virtues, meaning construction, and genre scripts, is what allows the integration of negative emotions into the aesthetic experience, modifying them into a pleasurable and rewarding affective outcome.

But what kind of pleasure or enjoyment is derived from or cooccurs with negative emotions? Kant has already described the aesthetic pleasure that we take in beauty as essentially different from the pleasure of mere sensory gratification, such as in eating or drinking (Kant 1951; Zangwill 2014). More recently, within the field of empirical aesthetics the pleasure from art has been linked with our "knowledge instinct" (Perlovsky 2010; 2014), namely, an inborn drive for cognition that "compels us to constantly improve our knowledge of the world" (Perlovsky 2007 , p. 73). In the broader field of affective neuroscience, pleasure has been distinguished into "core" and "conscious" pleasures (Brattico 2015; Kringelbach \& Berridge 2009). We have proposed a similar distinction for the type of pleasure that is experienced as a consequence of exposure to art objects (Brattico 2015). Core or sensory pleasure is immediately and involuntarily activated by the sensory properties of the stimulus, and it is associated with the fast firing of dopaminergic neurons of the mesolimbic and nigrostriatal pathways, which, in turn, modulate the neuroendocrine, visceral, and muscle responses of the autonomous nervous system, hence altering the bodily state. A slower, voluntary pathway, recruiting frontal lobe structures, mediates instead conscious pleasure or enjoyment. Also, in philosophical aesthetics, scholars postulate the pivotal role of contemplation, intentionality, and cognition in explaining aesthetic pleasure (Bundgaard 2015; Fenner 1996; 
Kemp 1999). In our previous work, we put forward a spatiotemporal road map of aesthetic processes related to music in particular, discerning between involuntary, low-level perceptualemotional stages and reflective processes involving cognitive control and leading to the three main outcomes of an aesthetic experience: aesthetic emotion, preference or conscious liking, and aesthetic judgment (Brattico 2015; Brattico \& Jacobsen, 2009; Brattico et al. 2009-2010; Brattico et al. 2013; Nieminen et al. 2011; Reybrouck \& Brattico 2015). These early and late processes are guided by what has been termed "focus" (Hodges 2016), "aesthetic stance" (Bundgaard 2015), and "aesthetic attitude" (Fenner 1996), namely, the intentional act of paying attention and preparing oneself for the contemplation and judgment of a work of art.

In cognitive neuroscience, experiments in which two tasks are contrasted with each other have probed the discernible neural mechanisms underlying aesthetic versus nonaesthetic modes of stimulus processing. For instance, the use of a visual paradigm comprising a task in which participants passively viewed abstract black and white patterns and another task in which participants were asked to contemplate the patterns aesthetically (to reflect on their beauty, but without giving an overt judgment) revealed that evaluative processes occurred during contemplation only and not during mere viewing, as indexed by the late positive electric brain response visible only in the contemplation condition (Höfel \& Jacobsen 2007). Furthermore, in this experiment the "not beautiful" patterns did not elicit the early frontocentral negative electric response indexing impression formation that has previously been observed in tasks involving overt aesthetic judgments (Brattico et al. 2010; Jacobsen \& Höfel 2003). These findings point to a separation between mechanisms for aesthetic mode, namely, the central processes of thinking about aesthetic value, and those related to deciding on an aesthetic judgment. In other neurophysiological experiments using auditory dual-task paradigms, early negative neural responses to aesthetic versus nonaesthetic tasks differed significantly from each other even before a cognitive judgment of correctness or an aesthetic judgment of beauty or liking was provided, and despite the fact that the same sound stimuli were listened to in both tasks (Brattico et al. 2010; Muller et al. 2010). These results indicate that an initial mode or attitude toward the object to be judged is taken, and this attitude conditions the neural mechanisms that prepare for subsequent aesthetic decisions. Similarly, neuroimaging studies consistently have demonstrated that the brain structures recruited during voluntary enjoyment of artworks, such as the orbitofrontal cortex, ventral striatum, thalamus, insula, and cingulate cortex, are separated from those associated with either unconscious pleasure or other basic emotions (sadness, happiness) (Brattico et al. 2016).

Hence, based on these arguments and empirical evidence, it is reasonable to propose that the intentional act to immerse in an art experience is a precondition for the enjoyment that might co-occur or follow negative emotions. Moreover, we suggest that this enjoyment is intrinsically judgmental; namely, it is glued with the compelling goal of providing a final judgment of liking, beauty, perfection, or similar. Without a proper attitude aimed at providing a final judgment on a work of art or fiction, the immediate response to a negative emotion could be a quick withdrawal, even when taking into account the Distancing factor. In the best cases, the outcome of this situation is a liking or aesthetic judgment, that can be (in various degrees) felt in the body and brain. In other cases, the outcome can even be detached from any feelings, namely, from any change in bodily state linked to neural activation of dopaminergic reward pathways, but instead be restricted to a mere cognitive decision of aesthetic value of an artwork, resulting in the willingness to repeat the experience in the future. And sometimes, a (positive) judgment is already taken even before looking at the (emotionally negative) painting, watching the (horror) movie, or listening to a (dissonant) musical piece, because of prior attitudes, culture, preferences (Gebauer et al. 2012), and group belonging (Wohl 2015).

\section{Artistic misunderstandings: The emotional significance of historical learning in the arts}

\author{
doi:10.1017/S0140525X17001625, e354
}

Nicolas J. Bullot ${ }^{\mathrm{a}}$ and Rolf Reber ${ }^{\mathrm{b}}$

${ }^{a}$ School of Creative Arts and Humanities, Casuarina Campus, Charles Darwin University, Darwin, Northern Territory 0909, Australia; ' ${ }^{\mathrm{b}}$ Department of Psychology, University of Oslo, NO-0317 Oslo, Norway. nicolas.bullot@cdu.edu.aurrolf.reber@psykologi.uio.no http://www.cdu.edu.au/creative-arts-humanities/staff-profiles/nicolasbullot

http://www.sv.uio.no/psi/english/people/aca/rolfreb/

Abstract: The Distancing-Embracing model does not have the conceptual resources to explain artistic misunderstandings and the emotional consequences of historical learning in the arts. Specifically, it suggests implausible predictions about emotional distancing caused by art schemata (e.g., misunderstandings of artistic intentions and contexts). These problems show the need for further inquiries into how historical contextualization modulates negative emotions in the arts.

Although the Distancing-Embracing model makes important contributions to our understanding of the enjoyment of negative emotions in art, it lacks resources to explain how misunderstandings in artistic communication modulate both negative and positive emotions. Specifically, the model misrepresents how artistic misunderstandings occur in several art forms. The Distancing-Embracing model and several other theories (e.g., Bullot et al., in press; Goodman 1968; Takahashi 1995) assume that artistic expression is a process of communication in which a sender (e.g., the artist) sends a signal (e.g., the work) to recipients, who interpret and respond to the work. Comprehension errors occur in this process. For example, receivers may misunderstand the artist's intention. More generally, recipients may err in comprehending the causal context of the work, which may lead to misunderstandings of artistic sources, provenance, and historical situations (Bullot \& Reber 2013a).

According to the Distancing-Embracing model, aesthetic appreciation of art forms that unfold in time, such as literature, film, and music, are interpreted by a two-step mechanism combining distancing and embracing processes. The model states that distancing is caused by the activation of schemata (Abelson 1981; Mandler 1984), including art schemata. Remarkably, the model posits that activation of an art schema necessarily causes a distancing effect. Most scholars would agree that activation of art schemata is critical in shaping responses to works of art. However, the model's description of art schemata fails to consider the roles of these schemata (1) in artistic misunderstandings and (2) in the contextualization of emotions.

First, by positing that art schemata are necessary conditions to distancing, the Distancing-Embracing model implies that artworks devised to prevent distancing are either systematically misunderstood as prescribing distancing (misunderstanding of an artistic intention) or systematically denied their art status (misunderstanding of an artistic context). However, there is no evidence for such systematic misunderstandings. This dubious prediction can be avoided by emphasizing the historical diversity of art schemata and by contextualizing the norms associated with these schemata.

Consider distancing. The model does not consider how the normative aspects of some art schemata aim to minimize emotional distancing. At least three categories of work customarily classified as art aim to minimize distancing and maximize emotional engagement: ceremonial and ritualistic artworks in first arts, edifying artworks in the religious arts, and works devised to cause social change by means of political activism or governmental propaganda. In these art forms, the classifications via art schemata are socially normative processes that operate by means of contextualization mechanisms (e.g., Bullot \& Reber 2013a; Kirk et al. 2009b; Swami 2013; Walton 1970). This normativity is noticeable in the social effects of these works. 
Take the example of schemata associated with first arts, such as the ornaments on warring shields and ceremonials aimed at daunting enemies. Aboriginal warriors did not intend to trigger their enemies' mental distancing from the fright caused by the perceptual experience of shields and war ceremonials. In addition to providing personal defense against blows, aboriginal shields functioned to express power and group identity and frighten foes (Jones 2007). Consequently, the normative functions of art schemata associated with these works were not aimed at the distancing of negative emotions.

Similarly, Christian art that depicted Jesus on the cross or the suffering of martyrs aimed at an immediate compassion unmediated by distancing; works of propaganda art aim at social persuasion unmediated by distancing.

A converse example of art intended to prevent embracing by eliciting disgust might be Damien Hirst's A Thousand Years, featuring a cow head infested with maggots. Danto (2003) commented on this piece: "someone told me that she found beauty in the maggots infesting the severed and seemingly putrescent head of a cow, set in a glass display case by the British artist Damien Hirst. It gives me a certain wicked pleasure to imagine Hirst's frustration if hers were the received view" (Danto 2003, p. 49). On Danto's account, because the artist intended the work to exclude aesthetic enjoyment, finding beauty in this work misunderstands Hirst's artistic intention. Although Hirst's artwork might show beauty despite its disgusting content (Menninghaus 2003), it is nonetheless possible in principle to devise an artwork that is intended to prevent or at least inhibit embracing. It would be a misclassification to exclude this work from the realm of art.

Second, the Distancing-Embracing model does not consider how understanding art is driven by sensitivity to cultural contexts and how this sensitivity modulates responses such as positive and negative emotions. Take the example of the film Triumph of the Will (1935) by Leni Riefenstahl. Triumph of the Will was devised to elicit embracing responses and, therefore, to minimize emotional distancing. Triumph of the Will first acquired cult status, but later became reviled as a piece of Nazi propaganda during the postwar period, a status that arouses negative emotions associated with distancing and lack of embracing. As noted earlier, the Distancing-Embracing model does not have the theoretical resources to predict and explain the historical variations in normative or emotional significance of this and other artworks.

In sum, several omissions prevent the Distancing-Embracing model from making robust predictions about artistic comprehension and the misunderstandings of emotions elicited by works of art. This problem is intensified by the authors' assumption that all artworks aim to produce aesthetic pleasure and enjoyment. This claim is contested in the philosophy of art (Carroll 2001; Davies 2006), and can be characterized as the aesthetic-artistic confound in the science of art (Bullot \& Reber 2013b). However, even from the standpoint of the authors' aesthetic theory of art, aesthetic valuation of an artwork ought not be the same when emotions are elicited by misunderstandings or by genuine historical comprehension. Taken together, these reasons suggest that the model would be strengthened by taking into consideration contextualization and historical change in the theory of (negative) emotions in the arts.

\section{Orange is the new aesthetic}

\section{doi:10.1017/S0140525X17001637, e355}

\section{Anjan Chatterjee}

Pennsylvania Hospital of Penn Medicine, Philadelphia, PA 19104.

Anjan@mail.med.upenn.edu

http://ccn.upenn.edu/chatterjee/anjan.html

Abstract: The Distancing-Embracing model proposes that negative emotions are constitutive of aesthetic experiences. This move is welcome and adds depth to empirical aesthetics. However, the model's emphasis on temporality challenges how best to think of static art forms. I suggest that "decisive" and "distilled" moments dilate time in the viewer's mind and might allow the model to accommodate photography and painting.

On a bright sunny day, under overwhelmingly blue South African skies, the farmer pulled out his knife and cut into the juicy orange. As I savored the delicious segment, he explained that his goal was to achieve the right balance of sweetness and acidity. A fruit that was only sweet would be boring and insipid. If only acidic, it would be unpleasant. The right combination of sweet and acid made for a rich and flavorful, one might say aesthetic, taste experience. In their Distancing-Embracing model of aesthetics, Menninghaus et al. develop this fundamental idea known to the orange farmer under overwhelmingly blue South African skies.

Empirical aesthetics has a long but thin history (Arnheim 1954; Berlyne 1971b; Fechner 1876). The field typically asked questions of preference in a fairly straightforward and perhaps insipid manner. In the mid-2000s, the field found new vigor with the addition of neuroscientific approaches (Chatterjee 2004a; Vartanian \& Goel 2004), as well as the articulation of early models of visual aesthetic experience (Chatterjee 2004b; Leder et al. 2004). This renewed vigor is evident in the accelerated number of publications in neuroaesthetics around this time (Fig. 1).

Models for empirical visual aesthetics continue to be refined and debated (Bullot \& Reber 2013a; Chatterjee \& Vartanian 2016; Jacobsen 2006; Redies 2007; Tinio 2013) and contribute to the evolution of this still nascent field. In the process, investigators became increasingly sensitized to areas that deserve further scrutiny. These areas include an understanding of the temporal dimensions of aesthetic experiences, as well as the role that negative affect and emotions play in positive aesthetic experiences (Chatterjee \& Vartanian 2016; Leder \& Nadal 2014). In addressing these points, the Menninghaus et al. proposal is welcome and overdue. Their central point is that acidity in the sweetness of aesthetic experiences is not unusual or extraordinary; it is precisely what makes aesthetic experiences intense, memorable, and rewarding.

On their account, various distancing mechanisms rendered by cultural conventions of what is framed as art or by dislocation in space and time allow artworks their alchemy in converting negative emotions to positive experiences. Much of the target article is about the dynamics of this conversion through an interplay of positive and negative emotions, the simultaneity of mixed emotions, and ways in which aesthetic sensorial qualities, meaning

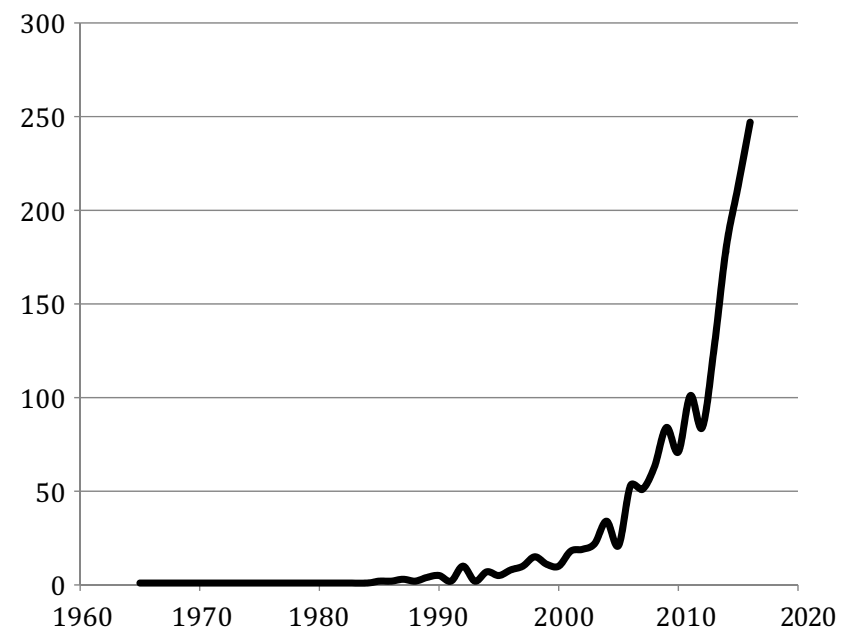

Figure 1. (Chatterjee). Number of published reports between 1965 and 2016 based on a PUBMED search for neuroaesthetics OR (neuroscience/neuropsychology AND art) OR (Neuroscience/ neuropsychology AND beauty). 
construction, and adherence to expected scripts mitigate pain to make way for pleasure. I have little to add to this comprehensive and insightful interpretation of aesthetic experiences. The model works best with art forms that unfold over time, such as literature, music, and cinema. But, do these ideas generalize to static visual arts such as photography and painting? In museums, people spend less than 20 seconds at images they like (Smith 2017), and undoubtedly, some of this time is spent in reading information about the art rather than immersing oneself in the image. How can such a short encounter do the work of embracing?

I suggest two ways in which the momentary expressions of, and encounters with, photographs and paintings could dilate time and lend themselves to the processing dynamics outlined by Menninghaus et al. Let me refer to these moments as decisive and distilled. The "decisive moment" is a phrase coined famously by the photographer Henri Cartier-Bresson (1952). It refers to a particular aesthetic of street photography of which he was a master. Events typically unfold with a beginning, a peak, and an end. The trick is to depict that peak moment that is informationally dense and hints at what came before and what comes after an impossibly thin slice of time. For example, Cartier-Bresson's famous 1933 photograph of children in Seville, Spain (see https://www.dodho.com/henricartier-bresson-collection-spain/) captures the delight of children laughing and playing, incongruously juxtaposed with the ravages of war depicted in rubbled homes and a hobbling boy. In viewing this image, one cannot help but feel mixed emotions of the kind outlined by Menninghaus et al. In the same vein, one cannot approach Picasso's Guernica (alluded to by the authors), painted around the same time and in response to related events as the CartierBresson photograph, without feeling its pain. This image represents a distilled moment. To experience the painting in its full force requires familiarity with cubist artistic conventions and knowledge of the historic events being depicted. Here, knowledge of the past and a certain visual literacy are part of the beholder's share that distills into the image the essence of an extended horrific narrative.

The aesthetic challenge for photographs and paintings then differs from those for art forms that extend in time. Photographers or painters who wish to include negative emotions to make their art more intense, memorable, and rewarding have to dilate time in the mind of the viewer, rather than modulate the experience within the already temporally extended forms of literature, music, and cinema.

\section{Reconciling an underlying contradiction in the Distancing-Embracing model}

\section{doi:10.1017/S0140525X17001649, e356}

\section{Gerald C. Cupchik}

Department of Psychology, University of Toronto, Scarborough, ON Canada M1C 1A4, Canada.

\section{Cupchik@utsc.utoronto.ca}

www.utsc.utoronto.ca/people/cupchik

Abstract: The Distancing-Embracing model proposes that negative emotions embedded in literary works can be rewarding. This is consistent with a holistic ontology in the German Romantic tradition. However, the application of cognitive psychology to explain experiences of aesthetic pleasure is problematic because it is founded on a mechanistic Enlightenment epistemology. The appreciation of negative emotions requires cognitive elaboration and closure, whereas hedonistic reward is contingent on the reader's needs, in the moment, for pleasure or distraction.

Menninghaus et al. describe a "paradox" whereby recipients choose to expose themselves to works that embody negative affect despite the potential for aversive emotional experiences. Their model incorporates "processing components" and/or "cognitive mechanisms" into psychology to account for "the pleasure in art reception that is associated with negative emotions" (sect. 1, point $\mathrm{C}$, para. 1). In everyday life, negative emotions secure attention, intensify emotional involvement, and enhance memorability, and these effects can transfer to aesthetic experiences. "Distancing" is initiated through the activation of "cognitive schemata of art, representation and fiction" (sect. 1, para 3) and is maintained throughout. This provides a sense of safety and control, and establishes a framework for "embracing" negative emotions, resulting in an experience that is "more intense, more interesting, more moving, more profound, and occasionally even more beautiful." A mixed array of stimulus properties involving "aesthetic virtues of using the media" and the compositional "interplays of positive and negative emotions," together with interpretive "meaning-making efforts," "integrate negative emotions into altogether pleasurable trajectories" (abstract).

Concealed beneath this "paradox" is a contradiction whereby a holistic and organismic ontology (concerning the nature of aesthetic experience) is paired with an atomistic and mechanistic epistemology from psychology to explain the underlying process. On the holistic side, they highlight Kant's idea that "the interplay of opposite feelings sets the mind of the spectator in motion." The dynamic interaction of positive and negative emotions leads to meaningful interpretations in accordance with what Fechner called a "reconciliatory moment." Thus, "meaning construction can redeem negative emotions on the level of higher cognitive processes" (sect. 1, point B, para. 2). The process of interpretation is influenced by metonymical contiguity such that the meaning of one element is modified by a neighboring element.

This is consistent with holistic ideas of the German Romantic movement. Johann Schlegel argued that, by reflecting the social realities of its audience, theatre can enhance social awareness. By selecting critical moments from life, and using carefully fashioned dialogue, the playwright exposes the hidden workings of a character's mind and shapes an audience's experience. Schiller proposed that aesthetic perception represents a way of relating to things that harmonize both thought and feeling, while involving the whole personality (Burwick 1991; Cupchik 2002). August Schlegel maintained that audiences combine a detached appreciation of theatrical illusion with an embracing and imaginative effort after meaning. Bullough (1912) anticipated the Distancing-Embracing model in his Gestalt account of optimizing "psychical distance." The "negative inhibitory" side involves detaching from the pragmatics of everyday life (i.e., Distancing) which is complemented, on the positive side, by an elaboration (i.e., Embracing) of the experience in a new aesthetic light. These various accounts emphasize the holistic interaction of audiences with art or theatrical works.

A problem arises when the atomistic Enlightenment epistemology underlying cognitive psychology is introduced to explain why negative emotions can shape aesthetic pleasure. The authors suggest that "artworks compete for attention" (sect. 2, para. 1) and "negative emotions are quite generally a resource that is predestined for the arts' purposes" (sect. 1, point A). Danziger (1997) has argued against the tendency to treat concepts "such as emotion or memory, as if they exist quite independently of how we think about them" because "the categories one meets in psychological texts are discursive categories, not the things themselves" (p. 186). Isolating "negative emotions" treats them as entities separate from the narrative situations in which artists and authors embed them. The idea that negative emotions are gratuitously inserted into texts falls within the Enlightenment emphasis on manipulating an audience's imagination and emotions through mimesis, the careful selection of subject matter treated as "real" (Schneider 1995). This self-conscious use of negative emotions to capture attention or shock audiences is appropriate for a subset of artistic projects or (soap) operas.

Reference to keeping "negative emotions at a cognitive appraisal-driven distance, thereby preventing them from being outright incompatible with the hedonic expectations of art reception" (sect. 6)) raises a series of issues. One has to do with relations between "emotions" and "hedonic expectations" or "reward." Arnheim (1985) took strong issue with "hedonistic psychophysics" - "the insipid and unfruitful aesthetic conception of art as a 
source of pleasure" (p. 861). He was against any attempt to reduce how "people perceive, organize, and comprehend works of art to a single scalable variable" (p. 861). The focus should be more on "persons who act as perceivers" than on the "objective percept" in isolation. This search for emerging meanings and metaphors (Arnheim 1971) fits with Winnicott's (1971) account of personally meaningful "transitional objects" in which emotion is invested leading to deep attachment. Thus, suggestions in creative works stimulate connections in recipients which determine attachment (Cupchik 2016).

Qualitative categories of emotion do not have an easy relationship with quantitative affective dimensions including pain-pleasure and arousal (Izard 1971). Happiness-sadness, fear-anger, and interest-disgust have been described as "natural-kinds" linked to our mammalian past (Panksepp 2007). Core affect and conceptual act theories (Barrett 2017; Russell \& Barrett 1999) explain emotion away in terms of nonemotional bodily processes linked to inferences about causal situations. A "cognitive appraisal account of emotions" (sect. 3.1) fits with this reductionist approach to explaining emotion in a way that is removed from holistic accounts of experience. This, in essence, captures the contradiction hiding beneath the paradox. Although the Distancing-Embracing model is consistent with Bullough's (1912) holistic account of "psychical distance," the isolation of negative emotion is burdened by limitations of an epistemology that denies the spontaneity of whole emotional experiences in favor of inferences about internal states based on logical analyses of situations.

The good intentions of the Distancing-Embracing model relate to a distinction between emotional elaboration and affective covariation in a depth of processing model (Cupchik 2016). According to the principle of emotional elaboration, a person pursues an exhaustive understanding of the work, embracing unique aspects of the narrative that resonate with episodic emotional memories. A deeper interpretation enhances attachment to and memory for the work (Garner 1962). The principle of affective covariation proposes that the work is appraised as having the potential for a positive or negative effect on pleasure or arousal, given a person's expectations and needs. Rewarding experiences thus result from coherent interpretations of situations or matching the chosen work against transitory affective needs, respectively.

\section{Distancing, not embracing, the Distancing- Embracing model of art reception}

\section{doi:10.1017/S0140525X17001650, e357}

\section{Stephen Davies}

Philosophy, School of Humanities, University of Auckland, Auckland CBD 1010, New Zealand.

sj.davies@auckland.ac.nz

http://www.arts.auckland.ac.nz/people/profile/sj-davies

Abstract: Despite denials in the target article, the Distancing-Embracing model appeals to compensatory ideas in explaining the appeal of artworks that elicit negative affect. The model also appeals to the deflationary effects of psychological distancing. Having pointed to the famous rejection in the 1960s of the view that aesthetic experience involves psychological distancing, I suggest that "distance" functions here as a weak metaphor that cannot sustain the explanatory burden the theory demands of it.

Some emotions - sadness and despair for example - are unpleasant to experience. We avoid circumstances that might occasion them when we can. But we do not always avoid some works of art, for instance, King Lear and Rigoletto, even when we know in advance that these are prone to elicit emotional reactions of the kind we normally shun (Levinson 2013).

This is widely discussed as the paradox of tragedy, and I use that term in the following. It is, however, worth noting that artworks that provoke horror (Carroll 1990a) and disgust (Korsmeyer 2011) fall in the same category. This is distinct from the paradox of fiction, which puzzles over the fact that we respond emotionally to characters that we know to have never existed (or that are long dead) (Davies 2009).

There are two main responses to the paradox of tragedy. They are different but not mutually exclusive.

The first argues that some benefit compensates us by outweighing the negative feelings we undergo. In its strongest form, this reply argues that the benefit could not be derived without our experiencing the negative; it depends on that experience. The benefit could be cognitive (e.g., Carroll 1990a) or affective (e.g., Aristotle 1972; Feagin 1983) or could come in the form of aesthetic pleasure.

The second response adopts a deflationary approach to the negativity of emotions aroused in the art setting. Berys Gaut (1993), for instance, argues that what is unpleasant about fear lies more in the actions it requires and in the consequences of those actions than in the way it feels as such. Where neither actions nor consequences follow for the audience engaged with a fiction, their negative response need not feel unpleasant. The somatic accompaniments of the response, such as the rush of adrenalin, can be enjoyed for the focus and alertness they bring on.

A particularly strong version of the deflationary view was presented by David Hume (1987). According to him, our interest in the story converts what would normally be an unpleasant reaction into a positive one. By way of explanation he appeals to what are supposed to be analogous processes -distance makes the heart grow fonder, we love the sick child more, nothing becomes a friend so much as his death. But on inspection these cases are far from similar and the comparison is unhelpful.

The authors of the target article do not locate their model within these approaches until the close of the article, and they seem to reject all of the options.

They deny that their model relies on compensatory mechanisms (sect. 4.6). Despite this, they catalog (sect. 2) how sad artworks produce easily recalled, vivid aesthetic experiences. "These emotional responses can be considered prime examples of high emotional intensity, high levels of aesthetic enjoyment, and high memorability as associated with artworks, the processing of which, includes marked levels of negative affect" (sect. 2, para. 7), a claim that strongly implies a mechanism of compensation. Moreover, the second factor of their model - "Embracing the distanced negative emotions for pleasurable purposes" (sect 4.6) surely amounts to compensation.

The authors also explicitly deny that theirs is a conversionary account (sect. 4.6). (But notice, then, how careless the labeling of Figure 1 is: "Solid rectangles indicate the processing components that are always involved in making negative emotions enjoyable.")

On its face, the favored model is deflationary (which, as noted earlier, is consistent with and facilitates compensation). The first factor in the model, the distancing of the negative emotion, is supposed to take the sting out of the emotion's negativity. Distance "open[s] experiential spaces in which negative emotions are not inevitably incompatible with art-specific expectations of hedonic reward" (sect. 3, para. 1).

Now, though, how convincing is the notion of "psychological distance" as an element in aesthetic experience? The notion was introduced into aesthetics in 1912 by Edward Bullough. $\mathrm{He}$ argued that the percipient had to psychically distance herself from the artwork to be in a position to appreciate it appropriately. With too little distance, her feelings could be overwhelmed; whereas too much would lead to indifference.

The authors of the Distancing-Embracing model appear to be committed to the centrality of psychological distance to aesthetic experience. They describe its mechanisms as involving situational awareness of one's separateness from the world of the artwork (sect. 3.1), and/or of the temporal and spatial discontinuity of its world with the real world (sect. 3.2), and/or of the fictionality of its contents (sect. 3.3).

I don't deny our awareness of such factors. Nor do I doubt that they can be relevant to our emotional responses to artworks, 
though the suggestion that these are less intense and more ephemeral than emotional reactions to real-world events is patently false in some cases. My worry is that the metaphor of "distance" is asked to carry the explanatory heft in the DistancingEmbracing model. Given that we do respond to what we know are not here-and-now situations, including to what we know to be fictions, it is not obvious that we are "distanced" from what we feel in a way that could solve the paradox of tragedy.

Beginning in the 1960s, philosophers of art (Cohen 1965; Dickie 1964) argued strongly against the idea that aesthetic experience involves a psychologically distinctive attitude of distancing. What was needed, George Dickie argued, was attention of the regular kind, plus knowledge of the conventions, history, and practices of the institutions within which art is made, presented, and appreciated. Despite some pushback (for example, see Hanfling 2000; Pandit 1976; Price 1977), these arguments succeeded in undermining the idea that aesthetic experience depended on an act of psychological distancing. Philosophers of art are not inclined these days to talk of psychological distance, except perhaps as a weak metaphor that could not perform the heavy lifting that the authors of the Distancing-Embracing model require of it.

\section{You are not alone - Social sharing as a necessary addition to the Embracing factor}

\section{doi:10.1017/S0140525X17001662, e358}

\section{Boris Egloff}

Department of Psychology, Johannes Gutenberg University Mainz, D-55099 Mainz, Germany.

egloff@uni-mainz.de

http://www.ppd.psychologie.uni-mainz.de/62.php

\begin{abstract}
I argue that the Embracing factor cannot be adequately conceptualized without taking into account the regulatory power of the social sharing of emotions. Humans tend to share their negative emotions with close others, and they benefit from it. I outline how this mechanism works in art reception by regulating and transforming negative emotions into positive experiences.
\end{abstract}

First, I share with Menninghaus et al. and all Behavioral and Brain Sciences readers the pleasure I experienced in reading this elegant and thought-provoking target article. In this comment, I argue that the very function of the Embracing factor, which "positively integrates, assimilates, or adopts the powers of negative emotions in the service of making art reception more emotional, more intense, more interesting, and, in the end, more rewarding" (sect. 1, point B, para. 1), requires an additional sixth processing component termed the social sharing of negative emotions. This argument is based on theories that emphasize the social function of emotions (Fischer \& Manstead 2008; Keltner \& Haidt 1999; Rimé 2007). In a nutshell, they argue that, as humans are social beings, emotions are essentially interpersonal; they signal inner states and action tendencies to other individuals. The act of sharing emotions with others is motivated by, for example, venting, seeking support, finding understanding, and bonding (see Rimé 2009). In turn, this leads to affiliation, enhances group cohesion, and ultimately serves the function of survival. Consequently, social sharing is a universal and often employed act of emotion regulation that is often met with subjective success. It is important to note that the social sharing of negative emotions in real life does not necessarily lead to recovery in the sense that the negative emotion is immediately and completely eliminated (Rimé 2007; 2009). However, this finding does not invalidate my argument of the importance of conceptualizing social sharing in the context of the enjoyment of negative emotions in art reception because (a) there should be severalquantitative and qualitative-differences among the emotions elicited by the arts and in real life, and (b) Menninghaus et al.'s model is "not a model of conversion, if conversion means a fullblown transformation of negative into positive affect" (sect. 4.6).

How does social sharing work in the process of positively integrating negative emotions into a rewarding perception of art? Please consider how often you attend places where you experience emotions elicited by the arts such as theaters, museums, cinemas, concerts, and readings, along with significant others (reading a book alone is certainly an exception to this rule). Please then remember how often and intensely you share your emotions with your companion immediately after the cultural event by talking about the emotions that this event elicited in you (not to mention the not so well-educated individuals who-to our displeasure-talk during the event). It is important that there are several additional nonverbal ways to share emotions in the form of crying together, consoling somebody, touching each other, exchanging glances, and so forth, that can also unfold during the event. Social sharing leads to affiliation, bonding, and relief, which are rewarding and definitely positive. As such, social sharing is at the core of transforming negative emotions into the enjoyment and pleasure of art reception.

It is interesting to note that this argument is in principle laid out in Menninghaus et al.'s article when they elaborate on empathy, compassion, and being moved in the case of sadness (sect. 4.2.1). These thoughts simply need to be transferred from an intrapersonal perspective to an interpersonal one: Person 1's sadness, which can be observed and is actively shared, leads to the empathic and compassionate actions of person 2 (and often vice versa), which, in turn, leads to positive feelings on both sides. (Anecdotal evidence says that at least for some individuals, the ultimate motivation to attend operas and watch movies is that it is fantastic to weep bitterly with your best friend.)

The same logic can in principle be applied to the benefits of social sharing in the cases of horror and disgust in art reception. In these cases, one can also think of an additional "social" component of impression management: To show significant others that you are not at all scared or that you enjoy being scared can have important interpersonal functions in terms of bonding and/or power, and subsequently, these experiences lead to pleasure (usually only for the actor in the case of power/dominance).

These assumptions can be put to empirical tests by assessing negative and positive emotions and indicators of the aesthetic enjoyment of art with or without other individuals. Specifically, one can systematically vary (a) the type of art (e.g., movie, play, painting), (b) the dominant negative emotion that it induces (sadness, fear/horror, disgust), and (c) the presence or absence of other people. Within the condition "presence," one can further differentiate among (i) the presence of a (any) person versus the presence of a significant other, and (ii) sharing emotions verbally and/or nonverbally versus not sharing versus suppressing.

Finally, I add that even when engaging in art reception alone, we can anticipate, remember, or imagine the act of sharing our inner feelings with close others, a process that should help regulate these feelings in a manner that is similar to the process outlined above. Taken together, as humans are social beings and emotions have important social functions, the social sharing of negative emotions is the key to regulating and transforming them into positive ones, also and especially in art reception.

\section{Boredom in art}

doi:10.1017/S0140525X17001674, e359

\section{Andreas Elpidorou \\ Department of Philosophy, University of Louisville, Louisville, KY 40292. andreas.elpidorou@louisville.edu www.elpidorou.net}

Abstract: In the light of recent findings on the nature of boredom, I argue that boredom is a potentially useful emotion in art reception and show how the Distancing-Embracing model can be applied to boredom. 
Boredom is conspicuously absent from Menninghaus et al.'s discussion. This is surprising. First, as a transitory affective state, boredom is an all-too-common experience. It affects individuals of all ages, genders, and cultures, and it does so in a wide range of situations (Acee et al. 2010; Fisher 1993; Game 2007; Grubb 1975; IsoAhola \& Weissinger 1987; Ng et al. 2015; Sundberg et al. 1991; Weinstein et al. 1995). Second, boredom is the topic of an active interdisciplinary research program. Its antecedents, effects, experiential profile, and neurophysiological correlates are all currently explored (Danckert \& Merrifield 2016; Eastwood et al. 2012; Fahlman et al. 2013), and there is strong evidence in support of the claim that boredom is an emotion in its own right (Van Tilburg \& Igou 2012). Third, a great deal of art is boring (Moller 2014). The cetology sections of Moby Dick are boring. Satie's Vexations, if played in its entirety, is boring. Wagner's Ring Cycle is boring. And so is Warhol's Empire, William Basinski's The Disintegration Loops, much of slow cinema, and many second movements of symphonies.

Although the authors do not discuss boredom, their remarks suggest that the Distancing-Embracing model does not apply to boredom. We are told that the compositional interplays of positive and negative emotions can lead to enjoyment because they render the experience of boredom less likely to occur. If the reduction or elimination of the experience of boredom is a desideratum of the Distancing-Embracing model, then boredom would seem to be a negative emotion that is incapable of enhancing aesthetic experience.

But if the Distancing-Embracing model does not apply to boredom, then the model fails to account for an experience that much of art elicits in audiences. Such a conclusion need not perturb the authors. The authors could respond that their model is not intended to apply to all negative affective experiences that arise within art, only to those that give rise to enjoyment. Assuming that boredom never leads to enjoyment, boredom falls outside of the scope of their model.

Although such a response is dialectically available, it might not be desirable. First, the authors would need to argue that the experience of boredom never gives rise to enjoyment. Second, the DistancingEmbracing model would offer an incomplete picture of our experience of negative emotions in art-an explanation of the role of boredom would still be needed. For those two reasons, I suggest a way of incorporating boredom into the authors' model. I argue that recent findings on the nature of boredom allow us to think of boredom as a potentially useful emotion in art reception, one that could promote an intense and focused aesthetic experience.

What is boredom? By "boredom" I mean the state of boredom (Elpidorou 2017b) and not the personality trait of boredom - the latter is conceptualized as the frequent experience of boredom in a wide range of situations, is measured using self-report scales (Farmer \& Sundberg 1986), and has been shown to be correlated with a number of harms (Elpidorou 2017a; Vodanovich 2003; Vodanovich \& Watt 2015). As a state, boredom is a concrete and shortlived affective experience that is characterized by feelings of dissatisfaction (Csikszentmihalyi 1975; Fahlman et al. 2013; Greenson 1953; Hartocollis 1972), attentional difficulties (Eastwood et al. 2012), and the perception of meaninglessness (Van Tilburg \& Igou 2012). In a state of boredom, one is disengaged with one's situation (Fahlman et al. 2013) and one wishes to do something else (Bench \& Lench 2013; Elpidorou 2017b; 2017c).

Recent work on boredom suggests that boredom is an emotion with a self-regulatory function. Because of its affective, cognitive, and volitional character, boredom can motivate the pursuit of a new goal when the current goal ceases to be attractive, meaningful, or satisfactory (Bench \& Lench 2013; Elpidorou 2014; 2015; 2017b; 2017c; Pekrun et al. 2010; Van Tilburg \& Igou 2011, 2012). Specifically, Van Tilburg and Igou $(2011 ; 2012)$ have argued that boredom not only makes one's activities seem meaningless, but also motivates one to re-establish a sense of meaningfulness. Indeed, boredom is capable of triggering meaning re-establishment strategies that affect an individual's behavior and cognition (Barbalet 1999). Furthermore, it has been shown that boredom can elicit nostalgia (Van Tilburg et al. 2013). Nostalgia can promote meaningfulness and is itself a bittersweet (although primarily positive) affective state (Routledge et al. 2012; Sedikides et al. 2008; Wildschut et al. 2006).

These two features of boredom-its capacity to promote meaning re-establishment strategies and its relationship to nostalgia-render boredom an emotion that is consistent with the Embracing factor of the authors' proposed model. First, boredom can motivate us to find or discover meaning in an artwork that previously failed to capture our attention. If we have no other option but to engage with the artwork, we will have to alleviate boredom by seeking alternative ways to interact with the artwork. Many contemporary works in theater, film, and music do precisely that: by not permitting easy solutions to boredom, they force us to return to the artwork and to try to uncover meaning. Boredom can thus produce a multilayered and cognitively demanding engagement with the work of art. What is more, by compelling us to discover meaning, boredom could lead to the favorable retroactive appraisal of the artwork (Oliver \& Woolley 2010).

Second, and somewhat more speculatively, just like other negative emotions (e.g., sadness and fear), boredom may also give rise to a concomitant feeling of a mixed affective nature that can reconcile the presence of boredom with our hedonic expectations of art reception. In the case of boredom, nostalgia could be the mediator emotion that transforms our experience and leads us to judge that our engagement with a boring work of art is not bereft of enjoyment.

The above considerations constitute only the beginning of an account of the role of boredom within art. Still, they underscore boredom's potential value in art and show how the DistancingEmbracing model can be applied to the case of boredom.

\section{Individual differences in embracing negatively valenced art: The roles of openness and sensation seeking}

\section{doi:10.1017/S0140525X17001686, e360}

Kirill Fayn and Peter Kuppens

Research Group Quantitative Psychology and Individual Differences, KU Leuven-University of Leuven, 3000 Leuven, Belgium.

kiril.fayn@kuleuven.be peter.kuppens@kuleuven.be

https://ppw.kuleuven.be/okp/people/Kirill_Fayn/

https://ppw.kuleuven.be/okp/people/Peter_Kuppens/

Abstract: We elaborate on the role of individual differences in the processing mechanisms outlined by the Distancing-Embracing model. The role of openness is apparent in appreciating meaning-making art that elicits interest, feeling moved, and mixed emotions. The influence of sensation seeking is likely to manifest in thrill-chasing art that draws on the arousing interplay of positive and negative emotions.

The Distance-Embracing (D-E) model of the enjoyment of negative emotions in art reception lays out a framework for understanding the paradoxical exposure to and enjoyment of negatively valenced artworks. But clearly, not everyone enjoys disturbing or unpleasant art. Although some people seek or relish it, others go to great lengths to protest or decry its existence and public display. While the authors acknowledge the existence of individual differences, the D-E model itself does not elaborate on the exact nature of such differences or how these differences should be understood in relation to known dimensions of personality. Yet, a better understanding of how people differ in their engagement with negatively valenced art is crucial for insight into the factors that lead to such engagement. In this commentary, we elaborate on the role of individual differences, focusing on the role of two traits - openness to experience and sensation seeking.

Both openness and sensation seeking are traits that describe approach tendencies, but toward partly different situations. Openness reflects cognitive exploration (DeYoung 2014) and is related to consumption of visual art, literary works of fiction, and classical 
concert attendance - meaning-making art. Sensation seeking, on the other hand, describes thrill and excitement seeking and is related to enjoyment of horror and thriller movies, disturbing and sexual art-thrill-chasing art.

Openness, as one of the five-factor model dimensions of personality, is not the usual suspect when it comes to predicting emotional experiences. Yet, this is unjustified, particularly in the aesthetic context. Below we identify three pathways of how openness connects to aspects of the D-E model. First, openness is the strongest personality predictor of powerful aesthetic experiences. For instance, open people are more likely to report chills, awe, and immersion and feel like crying in aesthetic contexts (Cotter et al., 2017; Silvia \& Nusbaum 2011; Silvia et al. 2015; Weibel et al. 2010). Thus, feeling moved is one mechanism from the D$\mathrm{E}$ model that may be responsible for the association between openness and engagement with negatively valenced art. This is supported by consistent evidence indicating that open people are more likely to enjoy sad music (Ladinig \& Schellenberg 2012; Vuoskoski et al. 2012) and appreciate negatively valenced abstract art (Rawlings 2003).

Second, open people's emotional reactions to art tend to be complex. In particular, mixed emotional states and evaluations, an aspect of the D-E model, are more frequently reported by open people both generally (Barford \& Smillie 2016) and in response to visual arts (Barford et al., under review). Further, open people have more nuanced emotional experiences with art, in that they tend to experience negative emotions in a more differentiated way (Fayn et al. in press-a). Thus, open people are less valence focused when it comes to engaging with negatively themed art, which may allow them to appreciate other aspects of artworks. Indeed, art expertise - a strong correlate of openness has been shown to predict focus on more stylistic, formal, and historical features of artworks (Parsons 1987). Such sensitivity and attentiveness to stylistic and formal features of art relate to the sensitivity to the aesthetic virtues aspect of the D-E model. Thus, open people may experience more mixed and nuanced states because of greater appreciation of the aesthetic virtues of negatively valenced artworks.

Third, openness is associated with a particular motivation for engagement with art. Although many people engage with the arts for the sake of enjoyment (a hedonic motivation), open people are more likely to engage with art with meaning-making motivations (a eudemonic motivation [Oliver \& Raney 2011]). This particular type of engagement is reflected in the meaning construction part of the D-E model. Interest in particular-a state associated with intellectual pleasure and eudemonic motivation - is crucial in understanding engagement with negatively themed art as it can be experienced independently from pleasure (Turner \& Silvia 2006). Because open people are especially prone to experiences of interest, and particularly interest in novel and complex art (Fayn et al. 2015; in press-b; Silvia et al. 2015), they may be more likely to engage and appreciate art with negative themes in the absence of pleasure.

Sensation seeking is similar to openness, in that they both represent approach tendencies, but the traits are distinct in terms of the types of experiences sought. Consequently, associations between aesthetic preferences and sensation seeking are similar yet distinct from those with openness. Sensation seekers, like open people, prefer complex and abstract visual art (Furnham \& Bunyan 1988). However, unlike openness, sensation seeking predicts liking violent and horror films (Aluja-Fabregat 2000; Tamborini \& Stiff 1987) and unpleasant representational visual art (Rawlings 2003). Thus, sensation seekers appear to enjoy a broader range of negatively valenced art, unlike open people, whose enjoyment of negative art is restricted to more highbrow art forms. As such, the influence of sensation seeking is more likely to manifest because of the increased arousal that negatively valenced art is capable of producing, as described in the interplays of positive and negative emotions aspect of the D-E model. Interestingly, both openness and sensation seeking independently predicted liking unpleasant abstract visual art, but not unpleasant representational visual art (Rawlings 2003). Open people may like the negatively valenced art because of its abstract and therefore complex nature, rather than the negative content, whereas sensation seekers may appreciate negative art regardless of type (abstract versus representational), because of the thrills and arousal such art can elicit.

In summary, we propose that individual differences are an important aspect of the D-E model, as not all people engage with negatively themed art. As such, already existing knowledge of the relationship between personality traits and art engagement is informative in understanding the processes that facilitate engagement with negatively themed art. Future work on the D$\mathrm{E}$ model should consider the influence of openness and sensation seeking, particularly with respect to selecting subjects and stimuli for future research.

\section{Empathy as a guide for understanding the balancing of Distancing-Embracing with negative art}

\author{
doi:10.1017/S0140525X17001698, e361
}

\author{
Gernot Gerger, ${ }^{\text {a,1 }}$ Tomohiro Ishizu, ${ }^{\text {a,b }}$ and Matthew Pelowski ${ }^{\mathrm{a}}$ \\ ${ }^{\mathrm{a}}$ Faculty of Psychology, University of Vienna, 1010 Vienna, Austria; ${ }^{\mathrm{b}}$ University \\ College London, WC1E 6BT London, UK. \\ gernot.gerger@univie.ac.at t.ishizu@ucl.ac.uk matthew. \\ pelowski@univie.ac.at \\ http://homepage.univie.ac.at/gernot.gerger/
}

Abstract: We connect the Distancing-Embracing model to theoretical and empirical evidence regarding empathy, which raises questions about the ordering and modulation of distancing in particular. Namely, distancing may not be a binary, continuously on/off process. Rather we suggest that changes in distancing as actualized via the relation between the individual and art (e.g., through empathy) might be a useful avenue for further consideration.

Menninghaus et al. (2017) provide a valuable conceptual framework for considering the paradox of enjoying negative emotions in the arts. This is an important step, which lays out two interrelated processes-distancing and embracing-and addresses a much needed topic of aesthetics and, indeed, provides a number of valuable hypotheses that can and should be investigated in future empirical and theoretical research.

The model suggests that a certain pre-state of activating an art/ aesthetic schema sets the stage for two sequential main processes: a "distancing factor," which invokes, or we would argue, implies, existential safety and "control," followed by a process of "embracing," which presumably is the main component of interest-providing visual pleasure and intellectual or hedonic enjoyment of art-and would presumably coincide with the main aspects of "mastery" or "savoring" in most discussions of art experience (cf. Pelowski et al. 2016). Especially negative emotions are argued to be enjoyed if and only if at least one component (from several subfactors) of distancing in concert with one component of embracing is activated.

We agree, and it is indeed supported by literature (e.g., Berlyne 1970; Kant 1963/1790; or discussions about the sublime: Burke 1757; Ishizu \& Zeki 2014), that intertwined processes of distancing and embracing might be a necessary precondition for better enjoyment of negative emotions. However, we also argue thatbeyond noting that art enjoyment should involve striking some balance between psychological or bodily distance from and perceptual engagement with a stimulus - the main research question may in fact start from this point: Namely, what are the necessary psychological mechanisms and conditions whereby distancing and embracing might be variably engaged? Relatedly, we might consider art engagement as recursive appraisal loops of pre-state, 
distancing, and embracing, which are tightly linked to momentary personal and emotional states. We especially consider the connection to empathy, based on recent evidence, which may be an important aspect to further consider in conjunction with the Distancing-Embracing model.

Here, we concentrate and elaborate on the recursive relation of continuous distancing (implied in the model) to embracing. A central aspect of the Embracing factor includes perceiving, experiencing, and regulating emotions (see the model in Fig. 1 of the target article). This set of processes can be modulated by empathy or the ability to feel into and understand one's own or another's affective states (Singer \& Lamm 2009). According to Menninghaus et al., such empathetic processes might be an important (although not necessarily sufficient) ingredient for a better experience of negative emotions, allowing individuals to experience emotions as an aspect of embracing them and, in negative cases, providing a self-gratifying experience that individuals can understand and perhaps control their (negative) emotional response (e.g., via meta-appraisals or conversion into mixed emotions).

A recent functional magnetic resonance imaging study by Ishizu and Zeki (2017), researching brain networks during enjoyment of emotionally negatively and positively valenced artistic photographs, supports the above argument. Both positive and negative photographs that were experienced as aesthetically preferable coincided with higher activation in brain reward areas. More importantly, only in the case of negative photographs was this higher activity modulated by areas dedicated to empathy, such as the supplementary motor area/medial cingulate cortex. Additionally, negative photographs coincided with activations in inferior frontal gyrus and inferior parietal lobule, areas related to emotion contagion (itself an empathy component). Elsewhere, participants with higher trait empathy or higher emotion contagion tended to report more intense (Baltes \& Miu 2014) and better aesthetic experiences for, as an example, emotionally negative music (Eerola et al. 2016; Garrido \& Schubert 2011). This would fit the model suggestion that "embracing" emotional content is a key aspect of enjoying negative art

As noted in the model, however, empathy alone might not be a sufficient condition to like negative art. This too was recently demonstrated by Gernot et al. (2017). ${ }^{2}$ In this study, participants high in emotion contagion and trait empathy showed more intense emotional "embracing" as indicated by self-reports and psychophysiological (facial electromyography, skin conductance responses) measures, but this did not result in higher enjoyment of negative art. Based on similar considerations Menninghaus et al. thus advocated an additional mechanism incorporating "being moved," suspense, or similar mixed emotion blends. Indeed, several studies report higher enjoyment of negative emotions through being moved in the case of sad films or music (Eerola et al. 2016; Hanich et al. 2014; Wassiliwizky et al. 2015). Interestingly, however, in Gernot et al. (2017), although high- compared with low-empathic participants also reported being moved more, they still disliked emotionally negative art.

This failure to obtain an increase in liking for negative art, even in conjunction with moving emotional experience, might be caused by an imbalance of distancing and embracing processes. High empathy may allow individuals to embrace affective content in art; however, if they are not able to regulate, distance themselves, or reappraise the elicited emotions, the overall aesthetic experience might remain negative. If, on the other hand, individuals can-even if they originally feel strong emotionsomehow conceptualize or "control" their experience, they might be able to enjoy negative art. This in our minds implies that distancing, in particular, is not a binary state engaged at the beginning of an experience, but rather is a continuously updated relation between the individual and art. Indeed, such an argument would fit with several theories involving enjoying emotional experiences in art (see, e.g., Cupchik and Wroblewski-Raya 1988; or Derryberry 1988). They suggest that processing can involve a switch within experience between an aesthetic and more felt pragmatic mode. This was also recently posited in a model of art experience by Pelowski et al. (2017), suggesting this active switch and the conditions whereby individuals may choose to modulate their distance from art. Equally, the evidence from the above empathy studies might suggest that some individuals cannot distance themselves. These aspects might be future candidates for model refinements.

\section{NOTES}

1. Corresponding author.

2. Please note that because of a production error, this work is cited as Gernot et al. (2017) (see erratum in Gerger et al. 2017).

\section{Live theatre as exception and test case for experiencing negative emotions in art}

\section{doi:10.1017/S0140525X17001704, e362}

\section{Thalia R. Goldstein}

Department of Psychology, George Mason University, Fairfax, VA 22030. tgoldste@gmu.edu

http://psychology.gmu.edu/people/tgoldste

Abstract: Distancing and then embracing constitutes a useful way of thinking about the paradox of aesthetic pleasure. However, the model does not account for live theatre. When live actors perform behaviors perceptually close to real life and possibly really experienced by the actors, audiences may experience autonomic reactions, with less distance, or may have to distance post-experiencing/embracing their emotions.

Live theatre is the only art form in which audiences engage with real humans, creating relationships, actions, behaviors, and emotions meant to be read as truthful. In much of contemporary theatre, perceptually, actors' behaviors are performed to be understood as close to real-life behaviors as possible. Authenticity is rewarded with critical approval, honors, and public admiration.

This perceptual authenticity has long led to popular beliefs about underlying inner reality: a mythos that there is truth under artifice (Benedetti 2007; Stanislavsky 1989). In certain mainstream styles of theatre, the combination of realistic perceptual cues while acting and popular conceptions of reality beneath performance may therefore cause audiences to react as if viewing real life, rather than as in an aesthetic space.

The Distancing-Embracing model endeavors to be art general and relies on top-down schemas to explain pleasurable negative emotions in art. Within distancing, the representation and fiction schemas allow audience members to separate themselves from artistically induced emotions, before experiencing the emotion itself. Within embracing, appreciation of aesthetic virtues allows audiences to welcome negative emotions specifically.

But the model does not account for watching live humans act out emotions, behaviors, and situations that look perceptually analogous to audiences' everyday experiences. Bottom-up automatic processes that occur in reaction to and in comprehending of such actions in real life may also occur while watching theatre (and, perhaps, only theatre). Therefore, autonomic contagious feelings of negative emotions may have to be reacted to, rather than prepared for (e.g., having a spontaneous, negative response to a real person standing next to you sobbing, and then reminding yourself that the person is just acting). Live theatre may additionally challenge the success of distancing and embracing processes in three ways.

First, live theatre erases the distance between "me" in the here and now and "art" in its time and place, considered key for the separation of momentary and reflective responses. Audiences in a theatre experience the play's events in real time, at the same time, in the same space as the actors. Newer theatrical movements in immersive theatre erase the distance of time and space more 
drastically. In many contemporary works, audience members sit on stage or walk around with the actors (e.g., recent productions of Spring Awakening, Sleep No More, Rocky [Brunner 2017; Dinesh 2017]). Actors in character interact with audience members, bringing them into the world of the play in a fully realized, $360^{\circ}$ experience. This erasure even disconnects theatrical acting from film or television acting.

Second, theatre (like all art forms) is not just art, but also something in itself. Visual arts, literature, dance, music, and film exist separately from the audience and create aesthetic depictions using metaphor and representation (canvas, written word, movement, musical note, screens). Live theatre is different. Unlike paint on canvas, or even musicians playing instruments or dancers moving in nonpedestrian ways, actors are not representations. Among heightened language, or elements of music and movement, the people in character are real.

Therefore, instead of creating aesthetic distance via fiction/representation, viewing actors may activate automatic processes that occur in the presence of real people. Any art general model must account for perception and reaction processes such as emotion contagion (Hatfield et al. 1994), mood contagion (Neumann \& Strack 2000), which occurs especially with negative emotions (Kelly et al. 2016), unconscious mimicry (Chartrand \& Bargh 1999), and efficient belief mentalizing (Apperly \& Butterfill 2009). These all occur in the physical presence of people, efficiently, immediately, and without top-down processes (Preston \& DeWaal 2002). Emotion contagion, in particular, is fast, reflexive, and uncontrollable (Hatfield et al. 2014), even when there is no goal of sharing emotions (Neumann \& Strack 2000). Theatre could activate all of these (and so may film [Coplan 2006]) before audience members have a chance to distance themselves. After experiencing autonomic emotions, audience members would then need to disengage.

Third, top-down processing of the aesthetic experience of theatrical acting may cause a lack of embracing. It is uncomfortable to watch someone wrestle with real mental illness, shame, or embarrassment (e.g., Krach et al. 2011). In the theatre, audience members may speculate that only an actor who is already troubled can portray mental illness (as, anecdotally, audiences already do for film and TV [Gerbner \& Gross 1976]), or that an actor can only portray joy, extreme sadness, or pain while actually experiencing it (e.g., Goldstein \& Bloom 2015; Goldstein \& Filipe 2017). Therefore, audiences may believe the emotions and behaviors they are seeing performed onstage are really happening to the actor, keeping them from finding pleasure in the experience. Although the sets, costumes, and lighting of theatre are certainly aesthetic, it is an open question whether humans, acting, are processed as such.

Given these features that only exist in live theatre, it can serve as an excellent test case. One way to test distancing, for example, is to systematically vary the realism of actors' portrayal and measure autonomic belief reasoning, emotion contagion, and other reactions. Research could compare audience responses with more or less realistic acting to investigate automaticity of emotional reactivity. Variance in the fictionality or representational nature of technical aspects of theatre could also test distanced versus bottom-up reactions. If realism makes a difference, automatic processes would occur more frequently and intensely for more (but not less) realistic portrayals. To test embracing via aesthetic appreciation, audience member belief (that actors must experience what they portray) could be manipulated before performance. If audiences believe actors must experience what they portray, they may have an inability to take pleasure in a "real" occurrence, when compared with believing they are watching pretense.

Level and type of representation, activation of automatic responses, and understanding of aesthetic virtues are different in theatre than other art forms, and call the generality of this model into question. Yet very little research has investigated live theatre or compared it with film, dance, music, and visual arts.
Manipulating theatrical factors would clarify the ordering of distancing and embracing and empirically test key components of this model.

\section{Fiction as a bridge to action}

\section{doi:10.1017/S0140525X17001716, e363}

\section{Melanie C. Green and Kaitlin Fitzgerald \\ Department of Communication, University at Buffalo, Buffalo, NY 14260. mcgreen2@buffalo.edu ksfitzge@buffalo.edu http://www.buffalo.edu/cas/communication/faculty/green.html}

Abstract: We propose an extension of the Distancing-Embracing model to the use of stories for prosocial ends. Specifically, audiences may find stories of individuals in need too emotionally overwhelming. Audiences may attempt to regulate or reduce negative emotions, which can reduce empathy and willingness to help. Through distancing, fictionalized accounts may counteract this tendency and thus increase prosocial behavior.

Menninghaus et al. have proposed a model that suggests that negative emotions gain attention and involvement for stories, but that the distancing mechanisms of art and fiction provide a means of safely enjoying such emotions. We suggest that these distancingembracing processes may have not only aesthetic effects, but also practical ones as well. Specifically, we believe that there may be an interesting extension of the authors' model to the use of stories for prosocial ends. Previous research suggests that individuals regularly learn and generalize from fictional stories (for reviews, see Slater 2002; van Laer et al. 2014). A possible implication of the Distancing-Embracing model is that fictional stories may be particularly useful in cases where true stories about challenging topics might evoke an overwhelming amount of negative emotion for readers or viewers.

For example, journalists or charities may share stories of individuals in need to inspire readers to help. However, a challenge that such efforts face is that readers (or viewers) may find the negative events described in a story too overwhelming. That is, they may feel personal distress or may not wish to be responsible for providing help. Readers may then engage in emotion regulation, shutting down their emotional reactions to the story. As a result, they can experience reduced empathy and reduced willingness to help. This process has been investigated in studies of the collapse of compassion, which show the ironic result that people are more willing to help a single individual than a large group in need (e.g., Cameron \& Payne 2011). A similar model, the Arousal: Cost-Reward model of helping behavior, suggests that people experience aversive physiological arousal when presented with someone in need of help (Dovidio et al. 1991). If individuals perceive helping to be costly, they may reduce that arousal in ways other than helping (e.g., avoiding the situation).

One implication of the Distancing-Embracing model is that persuaders may be able to use the Distancing function of art or fiction to increase prosocial behavior. For example, a reader may be presented with the story of a fictionalized earthquake victim. Because the story is fictional, a reader may be willing to fully empathize with the victim and experience the negative emotions that may accompany the description of hardship that the victim endured. In other words, the Distancing processes help avoid the emotion regulation that may otherwise reduce empathy. However, if the readers are then given the opportunity to help real earthquake victims (by donating or volunteering), they may be more likely to do so.

We have been investigating these emotion regulation processes in our lab in the context of restorative narratives (see Tenore 2014). Restorative narratives are stories of human tragedy or trauma that focus on the recovery and resilience of the individual (s) who has experienced the adversity (e.g., earthquake victim). We propose that by highlighting themes of hope and forward 
progress, the audience is not as overwhelmed with negative emotion, and the need to regulate emotion is reduced. In a series of studies, we compared restorative with nonrestorative versions of narratives about individuals facing adversity. In one study, we examined responses to a video about a man named Ruben who survived a devastating tornado. Participants who watched the restorative version, in which Ruben gets his dream job, reported a greater willingness to volunteer at a disaster relief organization than those who watched the nonrestorative version, in which Ruben loses his job because of posttraumatic stress disorder. In another study, we replicated this finding using written narrative versions of a news story (restorative, nonrestorative) about victims of Hurricane Katrina. Reading the restorative news story led to a greater willingness to volunteer at disaster relief organizations (Fitzgerald \& Green 2017). Moreover, those who read the restorative narrative reported experiencing more meaningful affect (Oliver et al. 2012), including emotions such as "touched," "moved," and "emotional."

In the studies described above, we did not vary whether the stories were described as fact or fiction (and, indeed, all of the materials were based on events that actually occurred). Rather, the content of the story influenced the need for emotion regulation. However, the Distancing-Embracing model implies that using a fiction label may be another route to helping individuals manage their emotions in response to stories with negative emotional content.

Suggestive evidence for this extension of the DistancingEmbracing model comes from another line of work in our lab. Beyond tragedy or trauma, another circumstance that may evoke negative emotion is reading stories about members of a potentially stigmatized group. In one recent study, we asked undergraduates to read a story about a female student who experienced events in her physics course that had the potential to evoke stereotype threat (e.g., a professor asking if she had help with the math on an assignment). Results suggested that individuals were more sympathetic to the student and more likely to recognize potential bias from her instructor when the story was labeled as fictional rather than factual, particularly in a firstperson version of the story. Labeling the story as fictional may have increased individuals' ability to empathize by creating emotional distance from the potential personal threats of either experiencing sexism or being accused of sexism (Green et al. 2017).

Of course, this proposed extension of the Distancing-Embracing model requires more direct empirical testing, and may have important boundary conditions (e.g., a request for help may be more effective if it does not come immediately after the narrative, to avoid reactance.)

The Distancing-Embracing model provides a valuable model of individuals' emotional experience while reading. We suggest that this model also may provide insight into certain types of persuasive processes; the distancing created by fiction may provide a pathway to embracing real others.

\section{ACKNOWLEDGMENT}

We gratefully acknowledge the support of National Science FoundationDRL Awards 1420036 and 1462063 for the research on reading stories about members of a stigmatized group.

\section{Art enhances meaning by stimulating integrative complexity and aesthetic interest}

doi:10.1017/S0140525X17001728, e364

\author{
Henrik Hagtvedt ${ }^{a}$ and Kathleen D. Vohs ${ }^{b}$ \\ a Department of Marketing, Carroll School of Management, Boston College, \\ Chestnut Hill, MA 02467; ${ }^{\mathrm{b}}$ Department of Marketing, Carlson School of \\ Management, University of Minnesota, Minneapolis, MN 55455. \\ hagtvedt@bc.eduｋvohs@umn.edu \\ http://www.bc.edu/schools/csom/faculty/bios/hagtvedt.html \\ https://carlsonschool.umn.edu/faculty/kathleen-vohs
}

Abstract: Menninghaus et al. portraved meaning in art as a vehicle for transforming negative emotions into pleasure. Although it is intuitively appealing that meaningful experiences should feel good, meaningfulness does not necessarily entail pleasure or positive emotions. Whereas easily comprehended art may elicit pleasure, meaningfulness is more closely tied to challenge and interest than to pleasure.

Consuming art can add rich benefits to the human experience. Menninghaus et al.'s exposition provides insights into an intriguing aspect of this phenomenon: art's capacity to make negative emotions enjoyable. Nonetheless, the notion of meaning as a vehicle for transforming negative emotions into positive ones is overly simplistic. This process may exist, but it is unlikely to be commonplace because of the nature of meaning and the forms of art likely to engender attempts to find meaning.

First, the notion that positive emotions flow when people find meaning in an event or stimulus has intuitive appeal, but recent analyses suggest a more complicated relationship. We studied happiness and meaning in life to assess their unique contribution to everyday feelings, concerns, and self-assessments (Baumeister et al. 2013). People rated how happy their lives are and, separately, how meaningful. We statistically removed the influence of happiness from meaning assessments and meaning from happiness assessments, thereby creating two "pure" residual variables representing each construct absent of the other. In contrast to Menninghaus et al.'s notions, meaningfulness was distinctly related to feeling stressed, worried, and anxious. These are not positive feelings. Meaningful engagements are more closely tied to negative emotions than the authors acknowledge.

Second, Menninghaus et al. neglected to delve into the qualities of artworks that encourage a search for meaning. We studied artworks of just that kind and contrasted them with artworks that demand less in the way of meaning analysis (Hagtvedt \& Vohs 2017). Using visual art that is easy versus challenging to immediately comprehend, we found patterns that conflict with the hypothesized relations asserted by Menninghaus et al. Our work showed that when art is easily comprehended, and therefore requires little meaning assessment, it stimulates positive feelings of happiness. In contrast, challenging art enhances the sense that life is meaningful.

Challenging artworks display elements that can be combined in intricate, ambiguous, or non-obvious manners. These are the formal qualities discussed by Menninghaus et al. When lines, shapes, and colors interact in a perceptually challenging painting, they form a complex but cohesive visual image that engages the mind more than a simpler depiction does (Lacey et al. 2011).

Our work illuminated two processes by which challenging art adds meaning (Hagtvedt and Vohs 2017). The first step is when art inspires exploratory, interconnected thoughts, which we conceptualized as integrative complexity (Tetlock et al. 1985). Integrative complexity is a way of thinking that encompasses both diversity in thoughts and reactions and coherence into a cogent whole. The formal qualities of challenging artworks were ideal for testing whether they inspire the experience of meaning in life through boosting integrative complexity. Our findings supported this notion. Challenging art, more so than straightforward art, elicited diverse reactions that were combined in new, non-obvious manners.

The second step of the process by which challenging artworks add to life's meaning was by provoking feelings of interest. Interest is an appraisal-based feeling akin to curiosity that arises from the perception of comprehensible novelty or complexity (Silvia 2008). We found that challenging art, more than simplistic art, led to feelings of interest, and interest, in turn, changed perceptions that life has meaning (Hagtvedt and Vohs 2017). Notably, interest is independent of pleasantness (Silvia 2008), which comports well with the aforementioned work demonstrating that experiencing pleasure tends to increase happiness, whereas meaningfulness is about engagement in meaty events and activities (Baumeister et al. 2013). These two complementary findings converge to imply that finding meaning in art is better characterized by interest rather than pleasure. 
The role of integrative complexity and interest in evoking a sense of meaningfulness underscores that the value of art is not reducible to simply feeling good. Art contributes to people's lives because it encourages an expanding scope of experience. As Menninghaus et al. argued, negative emotions can be attenuated or cast in a positive light when people reappraise the negative emotion content. Nevertheless, negative emotions may be an integral, unavoidable component of much of the art that broadens the mind and contributes to life's meaningfulness.

\section{Context matters: How macroeconomic forces may alter the reception of negative emotions in art}

\section{doi:10.1017/S0140525X1700173X, e365}

\section{Hal Ersner Hershfield ${ }^{a}$ and Adam Lee Alter ${ }^{b}$ \\ ${ }^{a}$ Marketing and Behavioral Decision Making Areas, Anderson School of Management, University of California, Los Angeles, Los Angeles, CA 90095; bStern School of Business, Marketing Department, New York University, New York, NY 10012. \\ hal.hershfield@anderson.ucla.eduａdam.alter@stern.nyu.edu \\ halhershfield.com \\ adamalterauthor.com}

Abstract: Menninghaus et al. offer a comprehensive model to explain why people pursue darker emotions in art, but we believe they underplay the considerable role of situational factors in driving these preferences. In particular, changing mood states are likely to shape artistic preferences, in large part because positive mood states act as a protective buffer against otherwise aversive experiences.

Menninghaus et al. propose a cogent and comprehensive model that explains when and why people enjoy darker or negative emotions when they perceive art. Although the authors include social, cultural, and situation variables as components in their model, they do so in an ancillary way: such factors are "likely to be only occasional contributors to such enjoyment" (Fig. 1). We contend, however, that these broader, situational variables meaningfully predict when people opt to consume heavier or more negative art forms.

To make this prediction, we draw on the affect and decisionmaking literatures. Previous research suggests that judgments and decisions can be influenced by aspects internal to a given decision maker (such as personality) as well as incidental emotional states brought on by external forces (e.g., the weather [Lerner et al. 2015]; for a comprehensive review, see Alter [2013a]). Notably, incidental states have carryover effects by which feeling states elicited by one context influence judgments and choices in another context (Forgas 2001). For example, anger that arises in one situation can prompt a motive to blame others in subsequent social interactions (Quigley \& Tedeschi 1996), and depression and sadness brought on by winter months can lead to increased risk aversion in the stock market (Kamstra et al. 2003).

Here, we assert that incidental mood states brought on by changes in broader situational domains, such as macroeconomic, political, and social arenas, may carry over to art reception. Past research has indicated that changes in such domains can in fact result in changes in public mood: Bollen et al. (2009), for example, found that daily fluctuations in the stock market were linked to daily fluctuations in the moods expressed by Twitter users. Could such changes, on a broader, more diffuse, level, lead to differential acceptance and consumption of differently valenced forms of art?

Mood theorists have suggested that mood can be thought of as a sort of "alarm system": Bad states indicate that something may be wrong and in need of fixing, whereas good mood states suggest to individuals that all is fine in their environment (Bless et al. 2006; see also Alter 2013b). Indeed, negative emotions often lead people to engage in behavior meant to alleviate an unpleasant state: Research participants who were induced to feel sadness chose smaller, immediate rewards over larger rewards that could be obtained only later (Lerner et al. 2013) and also opted to eat more "comfort" food (Garg et al. 2007).

By contrast, and perhaps more relevant to the Menninghaus et al. target article, research from the "mood-as-resource" literature proposes situations in which people might actually seek out negative information. Namely, researchers have proposed that positive moods are resources that act as a buffer against negatively valenced information (Trope et al. 2000; see also Alter \& Forgas 2007). As an analogy, Raghunathan and Trope (2002) liken positive moods to currency: Just as a rich person has more material resources with which to purchase expensive products that will nonetheless put a dent in his or her budget, someone who is "rich in positive mood" may feel less daunted by the possibility of interacting with or consuming negative material. In one study, research participants were willing to take in negative performance feedback, but only if they were in a good mood first (Trope \& Neter 1994). Theoretically, such negative information is not consumed without good reason. Information or material that can lead to better outcomes in the long run can sometimes be associated with short-term emotional costs (e.g., negative performance feedback that can nonetheless help one improve over time). Although such negative material can dampen mood, people who begin in a positive state have a buffer that allows them to process such negative information. One influential line of work-Fredrickson's (2004) Broadenand-Build theory of positive emotions - even suggests that positive emotions may be useful precisely because they allow for greater perspective and, ultimately, the incorporation of negative material. Along these lines, longitudinal research designs have found that people who frequently experience negative emotions alongside positive emotions (i.e., mixed emotions) experience fewer agerelated declines in physical health (Hershfield et al. 2013), and improved mental health if they happen to be undergoing therapy (Adler \& Hershfield 2012). Similarly, mixed emotional states foster more creativity in laboratory-based tasks (Fong 2006).

Taken together, this work implies that positive changes in macroeconomic, political, and social contexts could allow for more acceptance and consumption of negative emotions in a variety of art forms. Nonetheless, many questions remain. Though we have discussed broad trends, could acute events (e.g., acts of terrorism) affect the consumption and reception of negative emotions differently than prolonged changes in collective mood? Are some segments of the population more or less swayed by such trends and events? Finally, although we have grouped economic, political, and social events as members of one family of events, might each domain have stronger or weaker effects on art reception? These questions, coupled with the literature suggesting that mood states sway information consumption, suggest that situational factors-specifically macroeconomic contexts-deserve more weight in the model proposed by Menninghaus et al.

\section{The paradox of tragedy and emotional response to simulation}

doi:10.1017/S0140525X17001741, e366

\section{Patrick Colm Hogan}

Department of English, University of Connecticut, Storrs, CT 06269-4025.

Patrick.hogan@uconn.edu

http://english.uconn.edu/patrick-hogan/

Abstract: The insightful analysis of Menninghaus et al. could be deepened and rendered more systematic by recognizing that our emotional enjoyment of tragedy - and our response to fiction more generally-are versions of what happens with simulation. They derive from the operation and evolutionary function of simulation. Once we understand emotion in simulation, we largely understand emotion in tragedy (and fiction). 
Menninghaus et al. address a version of the "paradox of tragedy" (see Robinson 2009). We do not want to experience suffering, for example, grief, but we enjoy stories about grief, even when they make us weep. The article insightfully articulates eight factors to explain the paradox. But, as with any new theory of complex phenomena, there are problems with the current formulation. First, the eight factors appear diffuse, almost random, only loosely held together by the spatial metaphor of Distancing and Embracing. Moreover, the authors draw on a range of theories from psychoanalysis to Structuralist linguistics. A more satisfying explanation would integrate those factors into an account that relies on better understood, literally specified, cognitive structures and processes.

To some extent, the authors take up appraisal theory for their explanatory framework. That provides a degree of integration. But it may lead to other problems. Specifically, the authors explain the paradox of tragedy by reference to processes that may raise difficulties for appraisal-based accounts. For example, the fiction frame discussed by the authors as part of the Distancing metaphor, would seem to generate the "paradox of fiction" (see Robinson 2009), the problem that we know characters do not exist, but we weep over their (nonexistent) sorrow and rejoice over their (nonexistent) happiness. If emotion results from our assessment of an event's consequences for our well-being, as appraisal accounts have it (see Oatley 2012, p. 30), then it would appear that fictions should have no emotional consequences. Prima facie, our emotional response to fiction would appear problematic for appraisal theory. Of course, it is always possible for advocates of appraisal theory to contend that the appraisal process need not have bearing on our actual wellbeing or even our beliefs about our well-being. But then one wonders just what is being explained by appraisal theory and how it is being explained.

There are three points to be made in relation to these issues. First, the insights of the article might be more productively developed by reference to an account of emotion that takes elicitors for emotion to be concrete particulars given in perception, (emotional) memories, and simulations. The idea here is that the eliciting conditions for emotion are ultimately concrete and experiential. Appraisal may affect our emotions, but it does so not because of its logic, but because of the concrete emotional memories or simulations that it activates. This is why, for example, painful images of individual suffering tend to arouse our empathy, whereas statistics typically do not (cf. Bloom 2016 , p. 89). Simulation of concrete particulars may be an especially important and underappreciated process in emotional response. (For further discussion, see Hogan [2011].)

Second, and related to the first point, the Distancing-Embracing metaphor may be discarded if we have an adequate account of simulation. The paradoxes of tragedy and fiction are versions of problems that bear on counterfactual and hypothetical simulations. Consider what happens when we imagine dire outcomes of some unattempted action. We have an aversive response to those simulated outcomes. That aversive response helps to motivate us to avoid actions that would lead to those outcomes. Conversely, if we imagine desirable outcomes, we experience a degree of pleasure. That pleasure helps to motivate us to engage in the relevant sorts of action. In both the aversive and attractive cases, we have a version of the paradox of fiction. We feel emotion even though we are not experiencing real situations, but imagining unreal ones. This is not a paradox for the explanation of emotion in terms of concrete, experiential particulars. (For further discussion, see Hogan [2013].)

In the case of aversive simulation, we have in effect a paradox of tragedy as well. We engage in the simulation of tragic outcomes to our endeavors, despite the aversive quality of the experience, sometimes even dwelling on the utter awfulness of those outcomes. Prima facie, one would expect this to be a matter of reward system involvement, which drives "seeking" (as Panksepp $\&$ Biven [2012] put it), even in the absence of "liking" (on reward components, see Chatterjee [2014, p. 209]). There is at least some evidence for this in research showing the activation of the reward system in compassion (see Kim et al. 2009), which presumably involves simulation of the target's feelings and experiences.

In short, the paradoxes of fiction and tragedy are special cases of the emotional operation of simulation. The literary paradoxes are explained by the same processes as account for simulation.

Finally, this analysis is consistent with the evolutionary function of simulation. Specifically, simulation allows us to avoid danger and pursue opportunities with less risk, because we envision the consequences of our actions "offline." This is particularly important in the case of danger, because simulation involves no real threat. But simulation has this function only if it engages our motivation systems. The simulation of dangerous outcomes motivates our avoidance of actions leading to those outcomes precisely because it provokes our fear. At the same time, our emotional response to such simulated outcomes cannot be so aversive as to prevent simulation in the first place. Thus, evolution has calibrated simulation bearing on dangers to produce both aversive emotion and engagement (through reward system activation).

Of course, emotion in response to simulation must not be as strong as emotion in response to real experience. We see this particularly in the simulation of future pleasure. The enjoyment of such simulation must be less than the actual experience of pleasure or we would not be motivated to pursue the real pleasure. Fantasy would be as good as the real thing. Here, too, evolution has calibrated simulation, in this case producing pleasure, but also limiting it.

The aim of these comments is to suggest a more systematic and integrated account of the emotional response to fiction, incorporating the insights of Menninghaus et al. For example, simulation helps us to understand the cognitive processes adumbrated by the Distancing-Embracing metaphor. In addition, the two forms of evolutionary calibration help us to understand the function of those processes.

\section{"Negative emotions" live in stories, not in the hearts of readers who enjoy them}

\author{
doi:10.1017/S0140525X17001753, e367
}

\section{Vladimir J. Konečni}

Department of Psychology, University of California, San Diego, La Jolla, CA 92093-0109.

vkonecni@ucsd.edu

https://psychology.ucsd.edu/people/profiles/vkonecni.html

Abstract: The commendably ambitious project by Menninghaus et al. fails because its main connective tissue - "negative emotions" - is beyond the grasp of the authors' largely literary approach. The critique focuses on their treatment of the Paradox of Fiction, the neglect of the biological, adaptive nature of emotions, and the absence of convincing empirical support for key aspects of the proposed model.

Menninghaus et al. deserve plaudits for attempting to interweave rhetorical, literary, philosophical, and scientific issues that are of consequence in the art process. A multidisciplinary approach is indeed what addressing the nexus of creation, production, and reception of art requires. However, the project largely fails, not because it is too ambitious, but because of its choice and treatment of the main connective tissue - something called "negative emotions."

The principal purpose of the commentary is a critique of the authors' treatment of this concept, exemplified already in their opening sentence: "Enjoyment associated with negative emotions in art reception has been a central issue in poetics and aesthetics ever since Aristotle's theory of tragedy" (sect. 1). If already the first sentence manages to conflate a prototypical story's themes that involve the characters' anger, fear, and sadness with the readers' allegedly analogous "negative" emotional states (and even an empathetic one, pity), that is because the article continually confounds such key issues. 
Two preliminary remarks: Aristotle's catharsis (mentioned prominently in the article) is arguably not about the spectators' enjoyment of their negative emotions, but rather about the satisfaction that they experience because they have safely "purged themselves" of the hostility that had gradually built up because of adverse life events (Konečni 1991). Also, much classical rhetoric (also mentioned prominently) has actually very little to say about "negative emotions" with regard to either the orator's/poet's motives and themes or the recipients' emotional states. A well-known example is Longinus (or pseudo-Longinus, first or third century CE), whose text On the Sublime was influential in eighteenth-century Europe and continues to be widely discussed in American classicists' circles (see, for example, the translation and commentary by Arieti \& Crossett [1985]). One can safely claim that only with Edmund Burke (1759/1971, Pt V, sect. I) is the effect of "words" on "affections," if any, argued in depth and influentially.

Turning to the key issues: Much of the article obliquely revolves around what is known as the Paradox of Fiction (the Anna Karenina Paradox), first discussed in modern, post-Humean times by Colin Radford and Michael Weston (1975). Briefly, it refers to the readers' feeling sad about, or moved by, the sad fate of a nonexistent person, a literary character. Almost all of the many philosophers who have addressed this problem have invoked terms such as quasi-emotion and as-if emotion, and even denied it the status of a genuine paradox - based on their belief that the readers' state is only the real-life emotion's very pale analogue. However, here is the Menninghaus et al. position: "These terms [as-if, quasi-, pseudo-] evoke the notion (which we consider misleading) that art-elicited emotions may be somehow a species of inauthentic emotions" (sect. 1, point C, para. 2). (Significantly, the authors fail to return to this issue.)

But of course they are inauthentic - certainly so from the following viewpoint, which challenges some other key aspects of the article. Emotions such as fear, anger, sadness, and joy are subjective states that are caused by significant events involving threat to survival, struggle for limited resources, and bonding with, or loss of, a cherished mate or progeny. These events are predominantly social, involving real people. It is not surprising that the oral and written descriptions of such events have always been enjoyable, interesting, and, frequently, instructive to readers. Many accounts have described the emotions allegedly experienced by mythical or reallife characters, most of which have indeed been "negative," which explains my choice of emotion terms above - terms that reflect existential and adaptive concerns. Precisely for this reason, because of the massive cognitive, metabolic, and physiological investment required to sustain the major basic emotions, it follows that it would not be desirable for readers and listeners to experience the genuine emotions themselves. Wisely, they usually do not.

A related problematic issue is that Menninghaus et al., presumably striving to be inclusive with regard to the temporal arts, discuss music in the analogous vein. But here, again, many major philosophers (Noël Carroll, Peter Kivy, Nick Zangwill) are in agreement that the so-called "sad music" does not make listeners genuinely sad - in line, generally, with the views of people as otherwise diverse as Eduard Hanslick and Igor Stravinsky (Konečni 2008; 2013; Konečni et al. 2008).

Then, there is the authors' recruitment into their model of the concept of distancing as something of a conceptual novelty. In fact, it was probably first introduced into English-language aesthetics in the 1950s by the commentators of Bertolt Brecht's "epic theater" (Konečni 1991). But the authors' dilemma should be this: If the story-induced readers' state is a genuine, real life-like sadness, then distancing would be next to impossible to accomplish; and if it is a quasi-sadness, then there is no need for distancing. No one has seriously challenged the Radford and Weston (1975) view that our "sadness" about Anna's (or Mercutio's or Duchess of Malfi's) sad fate does not have any of the goal-directed or coping attributes characteristic of genuine emotions.

Finally, only three studies (Gerger et al. 2014, involving "affectively negative pictures"; Lundqvist et al. 2009, using "sad music"; and Wagner et al. 2016, with "anger-inducing performances") are cited by Menninghaus et al. to the effect that they report autonomic and electromyographic (EMG) changes in viewers and listeners. However, a close examination reveals that in all three studies there are major methodological shortcomings (see also Konečni 2015). The autonomic results are weak and hardly indicative of genuine emotions. As for the EMG findings, they seem to demonstrate the participants" "facial commentary," rather than genuine emotional experience. Such absence of links to solid and pertinent empirical work would seem to reveal the authors' analysis for what it actually is - a mostly literary handling of emotions. This is by no means intended as a condescending description, but rather as a warning that a predominantly literary analysis of the role of emotion in art runs into serious problems when it reaches beyond metaphor to handle psychological states with clear biological underpinnings.

\section{What is art and how does it differ from aesthetics?}

\section{doi:10.1017/S0140525X17001765, e368}

\section{Robert Kreuzbauer}

Surrey Business School, Faculty of Arts and Social Sciences, Rik Medlik Building, University of Surrey, Guildford GU2 7XH, United Kingdom. r.kreuzbauer@surrey.ac.uk http://www.surrey.ac.uk/sbs/people/robert_kreuzbauer/

Abstract: Art objects differ from other objects because they are intentionally created to embody a producer's (i.e., artist's) expression. Hence, art objects are social objects whose appeal and value are determined largely by the strategic interaction between the artist and the audience. I discuss several aspects of how strategic interaction can affect an art object's perceived value and aesthetic appeal.

A person's perceived value of an art object will always be influenced by the object's aesthetic appeal. But aesthetics is only one component of determining an art object's perceived value as the following examples illustrate: An identical poster print of a Mondrian painting would, by definition, have the same aesthetic appeal as the original painting, yet the former would have much lower perceived value and would also not be perceived as art (it merely represents a copy of an art object). Likewise, a software algorithm could generate an unlimited number of original "Mondrian" motifs. Some might even be more appealing, creative, or unique than those created by the artist himself. But again, only the paintings with the motifs designed by Mondrian would be considered as art and hence be perceived as having artistic value.

These two examples illustrate what makes art different from other forms of aesthetically appealing objects such as décor and entertainment objects. As we have shown elsewhere (Kreuzbauer \& Keller 2017; Kreuzbauer et al. 2015), art objects are intentionally made to embody a producer's (i.e., artist's) expression. In other words, the art object represents the materialised expression from the very moment when it was produced (i.e., the material object "freezes" a moment of time and space). Whereas Mondrian's original painting is a truthful representation of his expression in the moment of creation, the poster print would merely be its copy. Likewise, an expression can be performed only by a human being and not by a computer algorithm.

This shows that art objects are social objects, whose appeal and value are determined largely by the strategic interaction between the artist and the audience.

Besides determining whether the artwork truly embodies an artist's expression, psychological valuation and appeal towards the artwork depend mainly on the content it intends to communicate. For example, an artist might draw the content of a trash can to address the negative consequences of consumerism. It is possible that such kind of negative associations would lead to higher pleasure as predicted by the Distancing-Embracing 
model in the target article. But under the perspective of strategic interaction, this causal mechanism between negative emotions and appeal is less clear. Instead, perceived value should be determined largely by the interactive context. For example, some perceivers might find the artwork as highly appealing when the artist's message is consistent with their own political view. Others might instead find it highly conventional and cliché-like and might therefore infer that the artist's true intention is not to criticise consumerism, but mainly to make money for him- or herself. In the latter case, the audience should have a negative reaction towards the artwork as it believes the artist to be dishonest. This negative reaction towards the artwork would be caused largely by a lack of trust of the artist and not by the object's aesthetics.

On a macro level, artists would even use symbolic elements to coordinate groups to compete for influence and status. For example, postmodernism was a social movement against the hegemony of modernism. Postmodern artists who planned to challenge the dominance of modernism did therefore intentionally select countersignals (Klimek et al. 2017; Kreuzbauer \& Cheon 2015), that is, symbolic elements which are in total opposition to values and common symbols of modernism (e.g., huge ornaments or dysfunctional elements to challenge modernism's "form follows function" doctrine). The side on which a person stands within this "battle for social influence" affects the person's appeal and emotional impression towards postmodern art. As endorser of modernist values, his or her reaction towards postmodern art would obviously be highly negative, but this should flip for a supporter of the postmodernist movement.

To sum up, I agree with Menninghaus et al. that negative emotions can positively affect an art object's perceivers value and pleasure. However, when aspects of strategic interaction are taken into consideration, the outcome might vary. In particular, I anticipate that a person's emotional reaction depends on whether the art object is perceived as a truthful embodiment of the artist's expression. In addition, when art is used to question or challenge the status of a particular group, a perceiver's emotional reaction would depend on whether he or she is a member of this group or not. Identifying such basic mechanisms of strategic interaction (e. g., status competition through countersignals) would also allow us to specify social contexts. Rather than a claim that "context matters," it would allow us to determine causal mechanisms for specific contexts of strategic interaction (e.g., during status competition, signal $X$ would lead to reaction $a$ for members of group $w$ ). See Klimek et al. (2017) for an empirical test of the mechanism of counterdominance signalling in the context of musical style cycles.

\section{The enjoyment of negative emotions in the experience of magic}

\section{doi:10.1017/S0140525X17001777, e369}

\section{Jason Leddington \\ Department of Philosophy, Bucknell University, Lewisburg, PA 17837. jason.leddington@bucknell.edu www.jasonleddington.net}

\begin{abstract}
Theatrical magic is designed to elicit negative emotions such as feelings of vulnerability, loss of control, apprehension, fear, confusion, and bafflement. This commentary suggests that the Distancing-Embracing model can help us understand how the experience of magic can be aesthetically pleasurable, not despite, rather thanks to, some of the strong negative emotions it provokes.
\end{abstract}

In 1998, David Blaine changed the face of television magic by including documentation of spectator reactions as a core component of his TV special, Street Magic. Here is a typical scene:

Blaine borrows a quarter and apparently bites a chunk out of it. The two spectators scream, jump, turn away, cover their mouths, hide their eyes, laugh. Blaine then blows on the quarter and it magically restores to its original condition. More screaming and paroxysmic laughter: "I'm going home, I'm going home." "I think I'm going to have my baby right here.

Blaine's special made visible magic's power to provoke potent and complex emotional responses, including feelings of joy, amazement, wonder, surprise, vulnerability, loss of control, apprehension, fear, interest, curiosity, confusion, and bafflement. Yet, although cognitive scientists have long recognized that magic is worthy of attention (see Kuhn et al. 2016), there has been no systematic examination of the nature of the pleasure we take in watching magic performance. Indeed, although magic has been almost entirely ignored by philosophers, art critics, and art historians, scientists have typically treated it as a mere tool for learning about the mind by studying our susceptibility to deception. And though there is a trend toward treating the science of magic as a part of a general "psychology of wonder" (Lamont 2017; Rensink \& Kuhn 2015), the question why we enjoy magic remains largely unexplored. This commentary takes a step toward rectifying this by sketching how the Distance-Embracing (D-E) model might be applied to the philosophical analysis of the experience of magic developed in Leddington (2016). On the account recommended here, the experience of magic is aesthetically pleasurable, not despite, but thanks to, some of the strong negative emotions it provokes.

The core idea in Leddington (2016) is that the experience of magic is essentially aporetic: It involves a form of intellectual bafflement - and so, a form of cognitive failure - that follows from encountering an apparent violation of the laws of nature that we know is fake, but that we nevertheless cannot explain. (Effective magic performance should leave you saying, "I know it's a trick, but I don't see how it could be.") There are two distinct negative moments in this experience: (1) the apparent violation of our ordinary, yet well-founded expectations for how the world behaves (naïve physics); and (2) our failure to be able to explain this apparent violation (beyond noting that it's a trick). The question is then: How can an experience in which these moments are primary nevertheless be aesthetically pleasurable?

When magicians attempt to explain this, they sometimes appeal to a "compensation model," on which other theatrical elements, such as comedy or spectacle, compensate for negative elements (sect. 4.6). The basic idea is that, because it's "not fun to be fooled, ... it is one of the magician's first orders of business ... to add a spoonful of sugar to help the medicine of magic go down" (Swiss 2002). But the problem is that this cannot explain magic's distinctive appeal. After all, why not simply drop the magic and keep the theater? It is undeniable that many people enjoy the magic itself. Thus, making sense of magic as an artform requires explaining how intensely felt negative emotion can positively contribute to the enjoyment of a magic trick. The D-E model provides a plausible framework for this.

One Distancing and two Embracing factors seem to be essential to magic performance. First, witnessing a magic performance involves activation of the art schema (sect. 3.1). That the sign reads "Magic Show" allows us to feel a measure of safety and control in the face of what might otherwise be a frightening experience: encountering an apparent violation of natural law. The activation of the art schema is related to an embracing mechanism: the activation of the "magic" genre script. This contributes to "(re) appraising negative emotions [such as feelings of apprehension, loss of control, and bafflement] in a more favorable light" (sect. 4.5, para. 1). After all, a good magic show should disturb and baffle us! More interesting, however, is the idea that the experience of magic involves "concomitant mixed emotions ... serve as bipolar mediators for incorporating negative emotions into positive enjoyment" (sect. 1, point A, para. 3). This is where the D-E model is especially illuminating vis-à-vis the enjoyment of magic. Arguably, mixed emotions such as amazement, wonder, surprise, and curiosity "can inform and partly transform co-occurrent or adjacent feelings of" apprehension, loss of control, and bafflement "and, hence, lead to perceiving [them] ... as (more) enjoyable” (sect. 4.2, para. 2). 
This would explain not only how we can genuinely enjoy the magic that most disturbs and baffles us, but also why such performances can be so captivating, memorable, and emotionally powerful (sect. 2). (It is not uncommon for someone to remember in detail a magic trick witnessed decades ago and, when asked, to recount the experience with great relish.)

On the other hand, the other two Distancing factors - activations of representation and fiction schemas - are inappropriate for magic performance. Magic involves the apparent presentation of an impossible event as impossible (Leddington 2016). This means: Something that we know cannot happen seems actually to happen (contra the fiction schema) here and now (contra the representation schema). However, other Embracing factors, although inessential, are often active in the reception of magic and can facilitate our enjoyment of negative emotions. For example, magic can inspire reflection on the limits of human understanding (meaning construction, sect. 4.4), which can lead us to positively appreciate the feelings of bafflement provoked by the performance.

Finally, magic provides rich opportunities for empirical study of the role of negative emotion in aesthetic enjoyment. For instance, some magicians believe that blurring the line between illusion and reality - and thus, putting the art schema and magic genre script into question - can both increase the emotional power of a performance and amplify spectator enjoyment. Is this correct? The D-E model would seem to predict, "No." But this is something that can be empirically tested-and there are many other possibilities besides.

\section{Does art expertise facilitate distancing?}

doi:10.1017/S0140525X17001789, e370

\section{Helmut Leder ${ }^{\mathrm{a}}$ and Norbert Schwarz ${ }^{\mathrm{b}}$ \\ ${ }^{a}$ Faculty of Psychology - Cognitive Sciences Research Platform, University of Vienna, 1010 Vienna, Austria; ${ }^{\mathrm{b}}$ Dornsife Mind \& Society Center, University of Southern California. Los Angeles, CA 90089-1061 \\ helmut.leder@univie.ac.at norbert.schwarz@usc.ed \\ http://ppcms.univie.ac.at/index.php?id=2559 \\ https://dornsife.usc.edu/norbert-schwarz/}

Abstract: With respect to the Distancing-Embracing model, we discuss whether experts with well-developed and highly accessible schemata that lend themselves to distancing have initial affective reactions similar to those of novices, who lack access to well-developed distancing mechanisms, and whether differences between experts' and novices' responses are apparent only after distancing mechanisms have had a chance to do their work. We revisit findings from Leder et al. (2014) and discuss the role of mixed emotions and fluency.
Menninghaus et al. propose that a group of psychological distancing mechanisms can "satisfactorily explain the pleasure in art reception that is associated with negative emotions" (sect. 1, point C) when combined with at least one embracing mechanism. The schemata that are central to their conceptualization of distancing mechanisms require familiarity with art forms. Information processing models of art (Leder et al. 2004) highlight the role of previous experience and knowledge and suggest that the proposed distancing mechanisms are better developed and more accessible for experts than novices. Art experts, in turn, show more profound aesthetic experiences (Leder et al. 2012). These considerations predict that experts and novices will differ in their response to negative emotion-eliciting art. But at which processing stage will these differences emerge? Are experts' initial affective reactions similar to those of novices? Do differences between experts and novices emerge only after knowledge-based distancing mechanisms have had a chance to do their work? Or is the mere categorization of the emotion-eliciting stimulus as art sufficient to dampen experts' negative affective responses from the beginning?

In a study that bears on these issues, Leder et al. (2014) exposed participants with different levels of art expertise to positively and negatively valenced artworks and assessed their affective responses with facial electromyography (fEMG), capturing corrugator supercilii (frowning muscle) and zygomaticus (smiling muscle) activity. Participants also rated how much they liked the artworks, as well as their emotional valence and familiarity. The left panel of Figure 1 shows the corrugator activity over the 7 seconds of exposure to the artworks. Compared with positively valenced art (dashed lines), negatively valenced art (solid lines) elicited stronger negative responses. More important, compared with non-experts, experts showed attenuated physiological reactions with less extreme corrugator activations; they also self-reported less extreme negative affect and liked the negatively valenced art more. This expertise effect is consistent with the distancing mechanism proposed by Menninghaus et al. However, analyses of the temporal trajectory revealed only small temporal changes in emotion and no shift from negative to positive responses (within the 7 seconds). Importantly, the differences between experts and novices were apparent immediately after exposure and did not differentiate further over time. Menninghaus et al. note that their model is not a model of emotion conversion. Instead, negative emotions are assumed to be incorporated into positive enjoyment. In Figure 1, such enjoyment should be visible in increased zygomaticus response. However, the observed smiling response was not moderated by perceivers' expertise in this way, nor does it provide evidence for increased enjoyment as time passes. Although the observed expertise differences provide some support for the model, the temporal pattern is not easily compatible with the assumption of an initial negative affective

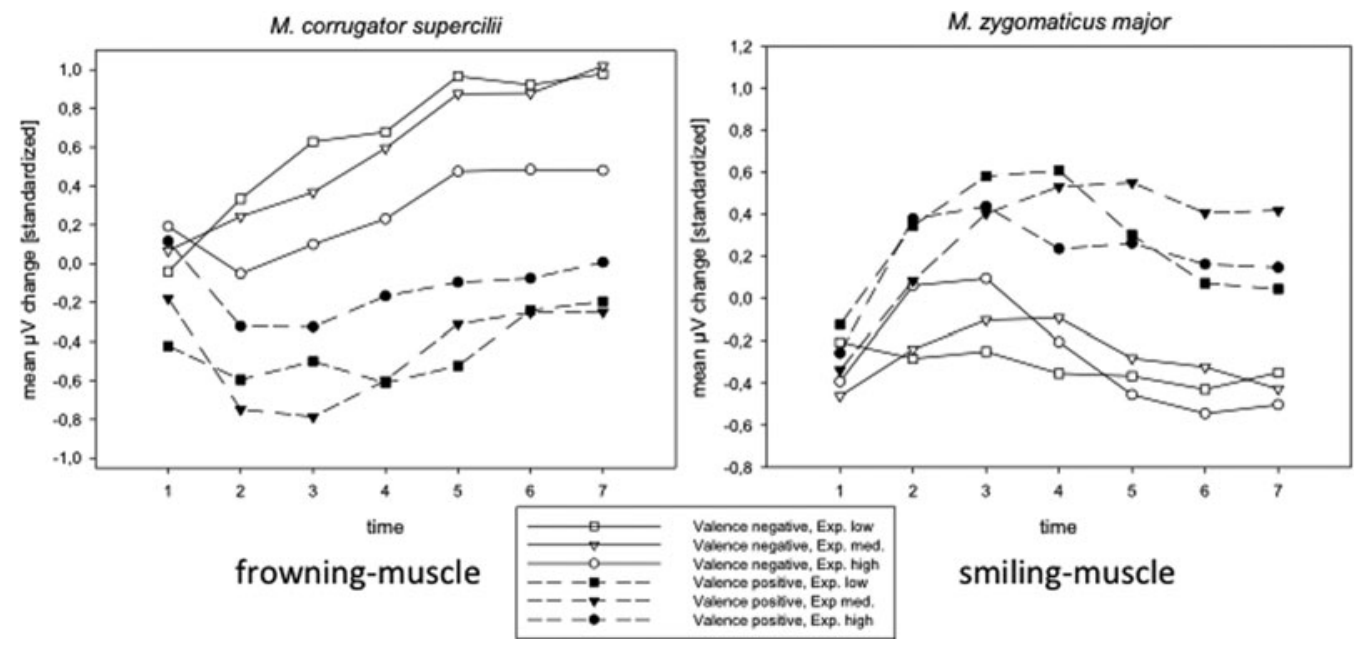

Figure 1. (Leder \& Schwarz). Facial electromyographic activity in response to artworks. Based on Leder et al. (2014). 
response that is overridden by the employment of cognitive distancing mechanisms.

The Leder et al. (2014) data were not collected to test the Distancing-Embracing model, although perceivers' art expertise and the physiological and self-reported affective responses assessed in the study are closely related to model variables. The noted ambiguities highlight that the empirical testability of the Menninghaus et al. model would benefit from clearer specification of what counts as supporting evidence, including measurement issues and the nature of the expected - and precluded - response trajectories. In our reading, a combination of physiological and selfreport measures (Forster et al. 2016; Gernot et al. 2017; Leder et al. 2014) seems promising. Note, however, that any measure attempting to capture the enjoyment of negative emotions in the context of art needs to heed the nature of "mixed" feelings, that is, the simultaneous presence of positive and negative responses. Unfortunately, many measurement attempts fail to do so. Mixed feelings can be identified only by assessing the presence and intensity of each feeling separately (e.g., "not at all" to "very much"). When this is done, even well-established "neutral" stimuli, such as the allegedly neutral pictures of the International Affective Picture System (IAPS), turn out to elicit mixed feelings (Schneider et al. 2016; Schneider \& Schwarz 2017). Without the ability to clearly identify mixed feelings and their respective sources, differential responses to different components of the experience may be misinterpreted as reflecting an integrative evaluation of the one component on which a given study happens to focus.

These measurement issues aside, we also note that complex assumptions about differential processing styles may not be needed to account for many expertise effects in art appreciation. A parsimonious model of aesthetic pleasure, Reber et al.'s (2004) fluency theory holds that the "more fluently perceivers can process an object, the more positive their aesthetic response" (p. 364). Fluency increases with repeated exposure, which is part of why an initial dislike for avant-garde art frequently turns into affection as the art form becomes familiar. Supporting this interpretation, repeated exposure to a disfluent art style has been shown to selectively increase liking for that style (Leder 2003). Repeated exposure to initially disliked art is also a key component of acquiring expertise through art education, and the resulting differences in processing fluency may account for many differences observed in the hedonic responses of novices and experts. From this perspective, the acquisition of schemata that are considered crucial for distancing is likely to be closely associated with differences in processing fluency that offer a more parsimonious account.

As these conjectures indicate, Menninghaus et al.'s (2017) Distancing-Embracing model provides a fruitful heuristic framework for the puzzle of why people enjoy negative emotions in the context of art. Its empirical testing, however, will require more precision with respect to the interplay of the numerous model variables, the temporal trajectory of distancing and embracing processes, and the assessment of the simultaneous experience of negative emotions and enjoyment. We look forward to future empirical tests and the further refinement of the model.

\section{Psychological models of art reception must be empirically grounded}

\author{
doi:10.1017/S0140525X17001790, e371
}

\author{
Marcos Nadal, ${ }^{\mathrm{a}}$ Oshin Vartanian, ${ }^{\mathrm{b}}$ and Martin Skov ${ }^{\mathrm{c}, \mathrm{d}}$ \\ ${ }^{a}$ Department of Psychology, University of the Balearic Islands, Palma de \\ Mallorca, Spain, 07122; ${ }^{\mathrm{b}}$ Department of Psychology, University of Toronto, \\ Toronto, ON M1C 1A4, Canada; 'Danish Research Centre for Magnetic \\ Resonance, Copenhagen University Hospital Hvidovre, Copenhagen 2650, \\ Denmark; ${ }^{\mathrm{d} C e n t e r}$ for Decision Neuroscience, Copenhagen Business School, \\ Copenhagen 2000, Denmark. \\ marcos.nadal@uib.es_oshinv1@mac.com martins@drcmr.dk
}

Abstract: We commend Menninghaus et al. for tackling the role of negative emotions in art reception. However, their model suffers from shortcomings that reduce its applicability to empirical studies of the arts: poor use of evidence, lack of integration with other models, and limited derivation of testable hypotheses. We argue that theories about art experiences should be based on empirical evidence.

Models are fundamental in any scientific field. They summarize and simplify phenomena that are otherwise extremely difficult to grasp, as is the case with the role of emotions - particularly negative emotions - in art appreciation (Silvia 2009; 2012). In this sense, Menninghaus et al.'s Distancing-Embracing model (DEM) is a welcome addition to the literature. But scientific models need to satisfy a set of criteria that establish their validity and ensure their potential to advance scientific understanding of the modeled phenomenon - in this case, the enjoyment of negative emotions in art reception. The validity and relevance of a model depend on the degree to which:

1. It summarizes systematically collected experimental or observational data: Models should be grounded on evidence.

2. It describes and explains the evidence: Models should highlight the causal mechanisms that bring about the evidence.

3. It formulates empirically testable hypotheses: Models should predict future evidence.

4. It brings theoretical clarity by integrating and relating diverse concepts and observations.

5 . It is compatible with models developed at other explanatory levels.

6. It clarifies its relation to other existing models.

Menninghaus et al.'s DEM does not fare well when measured against these criteria. First and foremost, the model is not motivated by a comprehensive body of empirical evidence. For instance, Menninghaus et al. produce only indirect and inconsistent evidence supporting the existence and engagement of the art, representation, and fiction schemata they suggest underlie the Distancing factor (sect. 3). The DEM predicts that these schemata, activated by art framing, should influence the experience of negative emotions. However, Wagner et al. (2014) and Gerger et al. (2014) - cited as supporting studies - found no such influence. The DEM is grounded not on empirical data but on assumptions about art and emotion derived from philosophical and poetic theorizing. One such assumption, presented in Section 2 , is that negative emotions are general resources predestined for the arts' purposes, implying that the experience of negative emotions is pervasive in art reception. This assumption, however, is clearly untenable, because not all interactions with art involve negative emotions (Martindale 2001; Smith 2014), and all forms of art, including prose and poetry, abound with works intended to evoke positive emotions (subgenres of comedy, erotica, lullabies, etc.). Moreover, no evidence is marshalled to bolster the paper's main claim, namely, that art's function is to transform negative emotions into pleasurable responses, nor to refute alternative possibilities.

Second, the DEM makes no reference, whether in cognitive or in neural terms, to the fundamental explanatory mechanisms underlying the distancing and embracing processes. Impressionistic descriptions are used to illustrate how, for instance, conceiving an art object as fictive can provide "an awareness that no real person (or animal) has been physically harmed" (sect. 3.3, para. 1). The notions invoked by such descriptions are conceptually obscure.

Moreover, the model's central concepts and processes, such as "experiential spaces" and to "keep felt negative emotions at some psychological distance" (sect. 3, para 1), are inadequately specified, bordering on folk-psychological. No attempt is made to relate them to psychological or neurobiological processes commonly studied by cognitive neuroscience. Does regarding something as fictive elicit top-down regulative mechanisms? What processes would such putative regulative mechanisms modulate? The perceptual processing of the percept being computed? The affective responses associated with these attenuated percepts? The DEM cannot answer such questions. And this is a crucial 
limitation, because in addition to describing a phenomenon, a scientific model should be able to explain how it works.

Furthermore, because the DEM does not postulate adequately specified explanatory mechanisms, the role of these mechanisms in the enjoyment of negative emotions cannot be tested empirically (sect. 4). For example, much is made about the co-occurrence of positive and negative emotions during the embracing phase. Testing this would require measuring the occurrence of negative and positive emotions separately and then registering their co-occurrence. Evidence from social neuroscience, however, suggests that rather than being dissociable, emotions are rooted in core affect - an organism's level of pleasant or unpleasant arousal (Wilson-Mendenhall et al. 2013) - and mapped onto a common reference space (Barrett \& Wager 2006; Barrett et al. 2007). Because the DEM does not lend itself to deriving testable hypotheses nor to falsification, its potential to motivate research in empirical studies of the arts is uncertain.

Finally, the DEM neglects existing models and explanations of the role of negative emotions in art reception (e.g., Sachs et al. 2015). For example, our understanding of visual art perception has been advanced recently by incorporating principles that account for the interplay between prediction error and reward (Kesner 2014; Van de Cruys \& Wagemans 2011). The basic idea behind predictive coding is that the brain actively anticipates incoming sensory input. When predictions are accurate, efficient processing of the input occurs. Conversely, when there is a difference between prediction and the actual state of affairs, a prediction error ensues. Prediction errors are therefore typically emotional and negative in valence. Van de Cruys and Wagemans (2011) proposed that artists habitually manipulate conditions that initially increase viewers' prediction error, which is subsequently resolved as the stimulus becomes predictable. This transition from unpredictability to predictability is experienced as rewarding. This account is consistent with evidence that visual perception has an affective component (Barrett \& Bar 2009) and that attaining conditions that favor processing fluency is pleasurable (Reber et al. 2004; Zajonc 1968).

In sum, because the model presented in the target article fails to meet basic validity and relevance criteria, it has limited potential to advance scientific understanding of the role of negative emotions in art reception. Without strong grounding evidence, clear explanatory mechanisms, and direct testability, the DEM is more a description of the phenomenology of art reception than a causal model that explains how negative emotions can lead to pleasurable engagements with art. What empirical aesthetics and neuroaesthetics require at present are models that make sense of the available mountain of empirical evidence and that make testable claims about neural mechanisms that can advance our understanding of how the human brain, as a matter of empirical fact, produces art experiences.

\section{Art as emotional exploration}

\section{doi:10.1017/S0140525X17001807, e372}

\section{Keith Oatley}

Department of Applied Psychology and Human Development, University of Toronto, Toronto, ON M5S 1V6, Canada. keith.oatley@utoronto.ca

http://sites.google.com/site/keithoatleyhomepage/

Abstract: The Roman poet Horace said poetry gives pleasure and instructs. A more informative theory is that poetry and art, in general, are less about pleasure than about exploration of emotions. Literary authors concentrate on negative emotions, seemingly to try and understand them. In two studies, reading literary art enabled the transformation of selfhood, not by being instructed but by people changing in their own ways.

The oldest surviving signs of art date back more than 100,000 years (Vanhaerren et al. 2006). They are of shells drilled to make beads, presumably for necklaces. What are we to make of such forms in human evolution? The first stone tools were scrapers, which emerged more than three million years ago. Like other technologies they were meant for purposes in the outer world, but the more recently emergent necklaces had other purposes. We may hypothesize that they helped people fashion their identities, to transform themselves within.

The Roman poet Horace (19 BCE/1932) proposed that the purpose of poetry and, by implication, all art is to give pleasure and instruct. We can see how wearing a necklace can give pleasure, but in their article, Menninghaus et al. point out that much art involves emotions that are negative. In this case, how can the first part of Horace's proposal be right? Why do people listen to music, look at paintings, go to see plays and films, and read poems, short stories, and novels, which concern negative emotions and which evoke negative emotions in them?

Menninghaus et al. say that, first, people know that although a work of art is not real life, it is in the world, so they can separate it and hold it at a distance, while, in their own everyday life, they can remain, as the authors say, in control. Second, while in this state, they say that people can decide to embrace a work. It is a valuable idea that in engagement with art, we can feel both safe and able to embrace the new.

The proposal can also be taken as an invitation to go deeper. Collingwood (1938) argued that art is exploration of emotions that we don't yet understand. It involves an externalization of an aspect of mind in the form of a language that can be of words, of painting, of sculpture, of music, of dance, and so forth. The externalization of consciousness in such forms enables exploration of a kind that is more difficult when concerns remain internal.

The reason art explores negative emotions, such as sadness in losing a loved one, anger at having been let down, as well as anxiety, shame, and disappointment in response to other kinds of upsetting events, is that such emotions are of the kind which are usually the most difficult to understand, which therefore need the most exploration, and which can have the most far-reaching implications for us.

Djikic et al. (2006) analyzed interviews of nine distinguished writers of fiction and nine distinguished physicists, using Pennebaker et al.'s (2001) Linguistic Inquiry and Word Count program. We predicted that the writers would use more emotion-related words as they discussed their work with interviewers, and this is what we found. We found, too, that as compared with the physicists, the writers used significantly more words related to negative emotions: anger, anxiety, depression, and sadness. As part of this study, we compared the interviews of the same nine physicists with a larger sample of interviews with 124 writers, and obtained similar results. So, in their work, literary artists have an inner preoccupation with negative emotions, which did not occur for physical scientists. This can be seen as confirmation of Collingwood's theory of art as exploration of emotions, with the addition that these emotions may often be negative.

In a piece of art that has been produced by someone else we, who engage with it, can also explore issues with which the artist is concerned-issues that can concern us, too. With a poem, short story, or novel, as with meditation, we can go to a quiet place and put aside concerns and preoccupations of daily life. For instance, by identification, we can take on the concerns and intentions of a literary character. Comparable processes occur with other art forms. In literary art, the emotions experienced are most often those of empathy with a character, in circumstances we may never have been in, but ones we can imagine. We may empathize with the character, but our emotions are not those of that imagined person. They are our own, in the circumstances of the story (Oatley 2016). In this way, we can lead many lives.

Menninghaus et al. ask whether greater emotional depth, with combinations of negative and positive emotions, might lead to greater dynamic change. We have explored this issue empirically. Djikic et al. (2009) asked people to read either a piece of art, Chekhov's (1899/1990) most famous short story, The Lady with the Toy Dog, or a control version that contained all of the same information, that was of the same length and reading difficulty, and that readers found just as interesting but not as artistic. Before and after 
reading, participants were given a test of personality, and also rated the intensity of 10 emotions they were currently feeling. As compared with those who read the control version, those who read Chekhov's story changed their personalities, significantly by small but measurable amounts, but not all in the same way. These participants changed in their own ways, and the changes were mediated by the amount of emotion change they experienced, across a range that included negative emotions, such as sadness, anxiety, and anger. Djikic et al. (2012) replicated the study with eight literary short stories and eight literary essays. Those who read a short story or essay that they rated as artistic changed their personalities, again idiosyncratically, in their own ways (see also Djikic \& Oatley 2014). The idea that relates, perhaps, to early necklaces, of art as potentially transformative, is here given further expression.

The proposals of Horace, that poetry and other arts function to give pleasure and instruct, seem to point in directions that are not as helpful as they might be. Is not the idea of art as exploration of emotional issues more informative than that of art as pleasure? Does not the finding that in reading literary art people can change in their own ways more psychologically suggestive than the idea that art is instruction?

\section{Art and fiction are signals with indeterminate truth values}

doi:10.1017/S0140525X17001819, e373

\section{Nathaniel Rabb \\ Department of Psychology, Boston College, Chestnut Hill, MA 02467. natrabb@gmail.com}

\begin{abstract}
Menninghaus et al. distinguish art from fiction, but no current arguments or data suggest that the concept of art can be meaningfully circumscribed. This is a problem for aesthetic psychology. I sketch a solution by rejecting the distinction: Unlike most animal communication, in which signals are either true or false, art and fiction consist of signals without determinate truth values.
\end{abstract}

Menninghaus et al. suggest that activation of the concepts <art $>$ and $<$ fiction> causes emotional distancing, a hypothesis that is both widely accepted in aesthetic philosophy (Kantian "disinterestedness"; see Stolnitz [1961]) and experimentally demonstrable (Gerger et al. 2014; Mocaiber et al. 2010; 2011a; 2011b; Van Dongen et al. 2016; Wagner et al. 2014). But their claim that < fiction > can be "activated far beyond the confines of art" (sect. 3) implies either that the concept <art> has meaningful boundaries for its possessor or the word "art" picks out a circumscribed set of objects. Both possibilities enjoy scant theoretical or empirical support. Normative art definitions face numerous difficulties (Davies 2006), and so far no evidence suggests that lay or expert intuitions converge on one or another (Kamber 2011). Our lab recently extended Malt's (1990) study on concept beliefs and found categories like "art" and "music" treated quite differently from other artifacts or nominal and natural kinds: their boundaries were considered the least strict, yet determined by experts to a degree on par only with biological groupings. Descriptive art definitions, on the other hand, seem reasonable but rule out very little. Most features on Dutton's (2009) list, for instance, arguably apply to nearly any complex human endeavor. We might shift the locus of explanation from illdefined objects to some special mental states associated with them, but plausible models of aesthetic experience face a similar predicament. Any reasoned judgment about an object or event likely involves sensation, perception, categorization, contextual knowledge, and affect (Chatterjee \& Vartanian 2014; Leder \& Nadal 2014) or these elements plus causal reasoning and considerations of object history (Bullot \& Reber 2013b; notably, Pearce et al. 2016, add disinterestedness to their list). This conceptual obscurity is not a problem for the authors' model but a considerable problem for aesthetic psychology, as the latter's objects of study are correspondingly vague. A possible solution expands the notion of fiction to encompass any signal believed to be neither true nor false.

In ethology, a signal is an action or property of an animal that guides another animal's behavior (Maynard-Smith \& Harper 2003). An index is a signal that cannot be faked, for example, intensification of display colors caused by healthy diets (Blount et al. 2003). Call these signals because they are always true (or reliable, if you prefer). Other signals can be true or false ( signals $_{\mathrm{TF}}$ ); a bird may issue a call that normally indicates predators whether or not a predator is present (Munn 1986). Although it is controversial whether nonhuman animals knowingly deceive, a signal's recipient must nonetheless judge its veracity before responding and can use contextual cues to do so (Heinen \& Stephens 2016). Yet humans seem (uniquely?) able to classify actions in a third way, as signals with no determinate truth value (signals $\left.s_{\mathrm{NDT}}\right)$. When someone yells "fire!" in a crowded theater, others must choose whether to flee or stay-unless the signaler is onstage in costume, in which case they understand that the action is not a claim about some occurrent state of the world. Invoking art or fiction through explicit labeling or contextual cues allows subsequent sensory inputs to be treated as signals ${ }_{\mathrm{NDT}}$. For organisms with the metacognitive capacity to represent beliefs and their relationships to reality, classifying input this way supports counterfactual thought ("suppose things were as she said"), thereby avoiding unnecessary action ("flee!") or erosion of trust ("she lied").

If correct, this view constrains investigation without specieswide art concepts or special aesthetic experiences. The objects of study are-minimally-things thought to be signals $\mathrm{NDT}_{\mathrm{N}}$. Although Menninghaus et al. call fiction a subgroup of public representations, fiction in this expanded sense is a superordinate category that includes and thus links the disparate artforms studied by aesthetic psychologists. Art and fiction are effectively co-extensive.

Tooby and Cosmides (2001; Cosmides \& Tooby 2000) have advanced a similar proposal in some detail, but restricted their discussion to public representations. Yet nonrepresentational art becomes especially intriguing on this view. There is obvious utility in our appetite for, and emotional responses to, fictional narratives, which illustrate social invariants from a safe remove (Mar \& Oatley 2008). But it is unclear why instrumental music or abstract art elicit any emotions when they have no narratives and represent nothing - yet they do (Gabrielsson \& Wik 2003; Pelowski 2015).

I have invoked ethology, but the capacity to classify input as signals $s_{\text {NDT }}$ need not be innate; it could be acquired across the life course, for instance, through pretend play (Weisberg 2015). One might worry that people initially treat all events as signals (Gilbert 1991), so the classification is simply illusory. But evidence runs contrary. Reduced amplitudes of an event-related potential associated with highly emotional stimuli have been observed for disturbing images presented as movie stills or artworks as opposed to documentary photographs (Mocaiber et al. 2010; Van Dongen et al. 2016). Comparable emotional attenuation has been captured with facial electromyography, functional imaging, and heart rate monitoring (Gerger et al. 2014; Mocaiber et al. 2011a; 2011b). A salient signal treated as true should not elicit attenuated emotional responses so quickly and consistently.

Of course, false signals ${ }_{\mathrm{TF}}$ would predict the same pattern. But people dislike deception. If artworks were considered mere lies, our enthusiasm for them should have long ago waned (although it remains possible that they are classified as "white lie" signals [Iñiguez et al. 2014]). Moreover, narratives explicitly labeled fictions nonetheless alter beliefs about the world (Green \& Brock 2000; Marsh \& Fazio 2006; Strange \& Leung 1999). That signals ${ }_{\text {NDT }}$ seemingly cause both emotional attenuation and belief updating is a tension ripe for study.

Why do we classify signals in this way? Some argue that the ability to represent counterfactuals is critical to all causal reasoning (Sloman \& Lagnado 2015; Weisberg \& Gopnik 2013). The attentional and mnemonic advantages of negative emotions that Menninghaus et al. emphasize foster engagement with signals $s_{\text {NDT }}$ despite the fact that these signals cannot literally inform. This 
Commentary/Menninghaus et al.: The Distancing-Embracing model of the enjoyment of negative emotions in art reception

engagement allows tuning of a vitally important faculty well into adulthood, when pretend play often cedes to propriety.

\section{Being moved is a positive emotion, and emotions should not be equated with their vernacular labels}

\author{
doi:10.1017/S0140525X17001820, e374
}

\section{Thomas W. Schubert, ${ }^{\text {a Beate Seibt, }}{ }^{\text {a Janis H. }}$ Zickfeld, ${ }^{a}$ Johanna K. Blomster, ${ }^{a}$ and Alan P. Fiske ${ }^{b}$ \\ ${ }^{a}$ Department of Psychology, University of Oslo, 0317 Oslo, Norway; ${ }^{\mathrm{b}}$ Department of Anthropology, University of California, Los Angeles, Los Angeles, CA 90095. \\ thomas.wolfgang.schubert@gmail.com \\ beate.seibt@psykologi.uio.no jhzickfeld@gmail.com johanna. \\ katarina.blomster@gmail.com afiske@ucla.edu \\ http://www.igroup.org/schubert \\ http://www.sv.uio.no/psi/personer/vit/beatesei \\ http://www.sscnet.ucla.edu/anthro/faculty/fiske/}

\begin{abstract}
As evidence for the second process of the Embracing factor, the target article characterizes being moved as a mixed emotion linked to sadness through metonymy. We question these characterizations and argue that emotions should not be equated with their vernacular labels.
\end{abstract}

The authors describe two emotions that they characterize as "mixed," being moved and suspense, as points in case for their second component of Embracing. We have studied being moved ourselves, and focus our comments on it. We feel that the evidence collected by ourselves and others does not fit with the characteristics of being moved that the authors propose, which challenges the second pillar of the Embracing factor of the model. So, what are those characteristics?

We found three relevant empirical claims in the model. The first is that being moved is a mixed emotion, supposedly meaning that it is defined as having concurrently both positive and negative affective components (cf. Deonna 2011). The second is that its relation to a corresponding negative emotion, sadness, is one of metonymy. The third is that sadness and being moved cluster in emotion space. We present evidence against all three claims.

The claim that being moved is a mixed emotion appears to be based on evidence gathered in studies that ask about emotional experiences retrospectively, often much later. Such data are useful for many purposes, but cannot clarify whether positive affect and negative affect were experienced concurrently or in succession. In a recent study, we concurrently and continuously assessed reports of being moved or touched, happiness, and sadness (among other variables [Schubert et al. 2016]). Across each of six different video clips, the time series of feeling moved or touched cross-correlated consistently and strongly with that of happiness. However, the cross-correlation of feeling moved or touched with sadness varied highly between clips and was very small overall. To the extent that happiness and sadness can be used as proxies for affect, these data are not in line with the idea that positive affect and negative affect consistently cooccur in being moved. (For a visualization of the data, see http://www.kamamutalab.org/visual/visual.html.)

The second claim assumes a relation of metonymy between sadness and being moved. As we understand it, this means that sadness is referred to as being moved and thereby acquires some of the positivity inherent in the mixed emotion being moved. However, the precise implication of the linguistic concept metonymy remains unclear from the theory presented. People do not always label their emotional experiences while experiencing them, and any assumption based on labeling would only be valid for a subset of experiences. Of course, there are transfer effects from one emotion to the next, as well as contrast effects. These are described in the first component of the Embracing factor, the dynamic unfolding of the story. In the case of being moved, well-orchestrated videos evoking being moved usually evoke some negative emotion first, often sadness, but sometimes anxiety or fear, which set the viewer up for then being moved. Here, being moved "profits from" sadness, and not the other way around, because being moved follows sadness rather than preceding it (see also Schubert et al. 2016). This profit is not due to metonymy, but rather the law of comparative feeling (Frijda 1988).

The third assumption is that being moved and sadness (related to social bonding and attachment) are highly associated and cluster closely together. According to our reading, cluster analyses on German vernacular emotion terms suggest that sadness does not cluster with being moved, but instead with other negative emotions. Being moved instead clusters closest with compassion, but also with nostalgia, admiration, tenderness, gratitude, and other positive emotion terms (Kinateder 2011; Menninghaus et al. 2015b; Schmidt-Atzert \& Ströhm 1983). The close relationship of being moved and compassion is corroborated by a robust correlation between the trait of empathic concern and feeling moved by a large variety of stimuli (Zickfeld et al. 2017).

Taken together, this implies that being moved is not a mixed emotion, but rather a positive one that enhances the enjoyment of art that elicits it. It does so by contrasting with negative emotions, especially sadness over the loss or suspension of social bonds and, thus, the process described in the first component of the Embracing factor.

The question remains why people feel moved and why being moved contrasts with sadness. According to our kama muta theory (Seibt et al. 2017), the relation of being moved and social sadness is one of polar opposites: Whereas being moved is elicited by the sudden intensification of communal sharing relations, social sadness is about their attenuation or loss. Communal sharing is a basic building block of human social relations (Fiske 1992). This hypothesis can account for the data presented in the target article. To get back to one of Menninghaus et al.'s examples: At funerals, people often oscillate between contemplating their loss versus their feelings of connection and. Thus. between grief and being moved. It is fully possible to feel both at once, or in quick succession.

One reason for our divergent perspective may be our belief that scientific conceptualizations of emotions should be distinguished from vernacular emotion terms and folk concepts. The use of the vernacular terms being moved, being touched, and their counterparts in other languages does not perfectly correspond to the emotional state caused by the intensification of communal sharing. Sometimes these terms are used for other states, and states that fit this appraisal are not labeled with them. Furthermore, neither does sadness match perfectly the state caused by the loss of a communal relation. We believe one cannot build theories on specific emotions by equating vernacular emotion labels with emotions themselves. Likewise, linguistic concepts such as metonymy may be applied to emotion labels, but not to emotions pars pro toto.

\section{Parental response to baby cry involves brain circuits for negative emotion Distancing- Embracing}

doi:10.1017/S0140525X17001832, e375

\section{James E. Swain ${ }^{\mathrm{a}, \mathrm{b}, \mathrm{c}}$ and S. Shaun $\mathrm{Ho}^{\mathrm{a}}$}

${ }^{a}$ Department of Psychiatry and Psychology, Stony Brook University Medical Center, Stony Brook, NY 11794; ' $\mathrm{D}$ Departments of Psychiatry and Psychology and Center for Human Growth and Development, University of Michigan, Ann Arbor, MI 48105; ' Child Study Center, Yale University School of Medicine, New Haven, CT 06520.

james.swain@stonybrookmedicine.edu

Shao-Hsuan.ho@stonybrookmedicine.edu

https://www.stonybrookmedicine.edu/profile?pid=2038\&name=James\% 20Swain\%20MD

https://www.researchgate.net/profile/Shao-Hsuan_Ho 
Abstract: The "art form" of parent-infant bonding critically involves baby conveying negative emotions - literally compelling parents to respond and provide care. Current research on the brain basis of parenting is combining brain imaging with social, cognitive, and behavioral analyses to understand how parental brain circuits regulate thoughts and behavior in mental health, risk, and resilience. Understanding the parental brain may contribute to solving the long-standing paradox of self-sought hedonic exposure to negative emotions in art reception.

Baby cry conveys profoundly compelling negative emotions, akin to those paradoxically present in art aesthetics (Hume 1757/1997) that secure attention, intense emotional involvement, and high memorability, yet invite care toward the development of the parent-infant bond (Swain et al. 2004). Parental brain mechanisms to regulate caring thoughts and behaviors are increasingly well understood (Swain \& Ho 2017a; Swain et al. 2014; Swain \& Lorberbaum 2008). Parents must hold the negative emotion perceptions at a cognitive-appraisal distance to also be compatible with the hedonic processing required for child care, that is, compassionate parenting (Swain \& Ho 2017b), which may be similar to those discussed in the Distancing-Embracing model of art reception.

By way of introduction, the "art form" of parent-infant attachment is also a landmark conceptualization of human developmental psychology (Bowlby 1969; 1973). From an evolutionary perspective, attachment represents the innate neurobiological systems promoting proximity seeking between an infant and a conspecific attachment figure - usually the parent. This proximity and contingent care, driven by the thoughts and behaviors of parents, increase the likelihood of infants surviving to reproductive age. The baby's survival depends on conveying negative emotions - often with a cry - associated with needs for safety, food, warmth, or other care. Over the last 15 years, brain imaging studies have used baby-cry stimuli to probe for mechanisms of adaptive parenting among mothers (Kim et al. 2016) and fathers (Swain et al. 2014) in mental health, in illness (Moses-Kolko et al. 2014), and in risky situations such as poverty (Kim et al. 2014). We discuss some of this parental brain research, which includes aversive infant stimuli such as baby cry, which may inform the Distancing-Embracing model of art reception.

Parent-infant attachment is another "art form" for which the aesthetic is very much in the "eye" of the beholder-in which parental brain activity depends on observed mental state talk (Hipwell et al. 2015). How parents perceive their child is also related to their early-life experience and peripartum circumstances that affect parental brain physiology (Swain et al. 2014). For example, high quality of perceived maternal care in childhood was associated with increased gray matter volumes in regions involved in regulation of emotions and social and sensory information processing, including the superior frontal gyrus, the orbitofrontal cortex, the superior and middle temporal gyri, and the fusiform gyrus (Kim et al. 2010b). Furthermore, higher levels of neural responses to infants' cry sounds were found in these same regions. The only region that was more active for mothers of low-quality maternal care in childhood was the stress-sensitive hippocampus. Perhaps early-life parental care also disrupts the appreciation of art with negative emotional content. In another study, early postpartum circumstances also altered brain structure (Kim et al. 2010a). Several brain regions involved in maternal motivation and reward processing, including the striatum, amygdala, hypothalamus, and substantia nigra, exhibited structural growth from the first few weeks to the fourth month postpartum. Structural growth was also observed in areas involved in processing sensory information and empathy, including the superior temporal gyrus, thalamus, insula, and pre- and postcentral gyri. Finally, regions associated with regulating emotions, such as the inferior and medial frontal gyri and the anterior cingulate cortex, also showed structural increases. This suggests that neural plasticity occurs in a wide range of brain regions, each serving important aspects of child caregiving in human mothers during the first few months postpartum. Furthermore, the greater the observed structural growth in the midbrain region (involved in reward and motivation), the stronger were the positive emotions a mother reported having about her baby in the third and fourth months postpartum. Researchers have yet to examine the strength of negative emotion perception directly, but this may already be a form of adaptive emotion distancing and embracing.

The evident plasticity may help explain psychopathology and treatment affect parenting. In a self-focused baby-cry task designed to provoke brain responses in participants with a history of adverse early-life experiences sometimes described as a malevolent background "shark music," amygdala reactivity was increased (Swain et al. 2017). Furthermore, time-dependent reduction in parenting stress was related to concomitant increased child- versus selffocused baby-cry responses in amygdala-temporal pole functional connectivity, which may facilitate maternal ability to take her child's perspective while maintaining appropriate distance.

One recent study begins to suggest how parenting brain mechanisms rooted in responses to baby cry during the development of the parent-infant bonding art form may be practically connected to later child outcome (Kim et al. 2015). In this study, relationships between parental thoughts/actions and brain responses to own versus other baby-cry stimuli among mothers and fathers in the neonatal period were studied in relation to the child's social and emotional development by toddler age. Results differed for mothers and fathers, suggesting sex differences in the brain basis of early parental brain baby-cry responses linked with child socioemotional competency outcomes. This suggests sex differences in the brain areas that may also ultimately fit into the Distancing-Embracing model.

Some of these complex neuroimaging findings may ultimately need to be interpreted as changes in distributed network function. For example, compared with maintaining one's emotional responses, engaging in reappraisal - a form of distancing-embracing neuroimaging task-produced robust and distributed alterations in functional connections involving visual, dorsal attention, frontoparietal, and default networks (Sripada et al. 2014). Indeed, a widespread set of brain responses were also reported in a recent study of mothers responding to child visual feedback after a caring decision (Ho et al. 2014). Responses that correlated with dimensions of empathy included the amygdala, ventrolateral prefrontal cortex, and supplementary motor area that may also fit with broader work on altruistic behaviors (Brown \& Brown 2015; Swain et al. 2012), which may also involve appropriate Distancing-Embracing of negative emotions.

\section{ACKNOWLEDGMENT}

The authors are supported by the Department of Psychiatry at Stony Brook University, National Alliance for Research on Schizophrenia and Depression.

\section{Genre scripts and appreciation of negative emotion in the reception of film}

doi:10.1017/S0140525X17001844, e376

Ed S. Tan ${ }^{\mathrm{a}, \mathrm{b}}$ and Valentijn T. Visch ${ }^{\mathrm{c}}$

${ }^{a}$ Department of Media, Cognition and Communication, Faculty of Humanities, University of Copenhagen, Copenhagen Dk2300, Denmark; ${ }^{\mathrm{b}}$ Amsterdam School of Communication Research, University of Amsterdam, Amsterdam 1018 WV, The Netherlands; ${ }^{\mathrm{c}}$ Faculty of Industrial Design Engineering, Delft Technical University, Delft 2628 CE, The Netherlands.

e.s.h.tan@uva.nl V.T.Visch@tudelft.nl http://mcc.ku.dk/staff/?pure=en/persons/545153

http://www.uva.nl/en/profile/t/a/e.s.h.tan/e.s.h.tan.html

https://www.tudelft.nl/io/over-de-faculteit/persoonlijke-profielen/ universitair-hoofddocenten/visch-vt/

Abstract: The Distancing-Embracing model reserves a role for genre scripts in the hedonic valuation of negative emotion in art. Genre scripts, as defined in the target article, leave higher-level recipient intuitions out of scope. We argue that, in film genre scripts, lower-level stylistic features lend access to more complex conceptual knowledge, 
including pragmatic principles. The argument implies a consideration of the communicative dimension of aesthetic works, which could strengthen both factors of the Distancing-Embracing model.

We find Menninghaus et al.'s hypothesis that activation of genre scripts contributes to (re-)appraising emotions in a positive light a novel and major contribution to understanding negative emotion in the reception of artworks. In this contribution, drawing on our research in the cognition of film genre, we comment on its underlying rationale.

Menninghaus et al. state that genre categorization on the basis of subtle stylistic cues has not been tested to contribute to effects on the processing of negative emotion. However, in a study not referred to in the target article, that is, Visch and Tan (2009), we did investigate the negativity of emotions across genre categorizations prompted by subtle stylistic cues. We found that all negative emotion labels we measured, namely, sad, impressive (in the sense of threatening), and scary, correlated positively with aesthetic liking, whereas the only positive emotion, funny, did not. This result seems to be in keeping with the hypothesis proposed by Menninghaus et al. Subtle stylistics cue genre scripts, and genre scripts have the potential to issue affective forecasts preparing the ground for a positive appraisal of otherwise negatively valenced emotions.

We present here an additional elaboration of Menninghaus et al.'s reappraisal hypothesis for the case of negative emotion in film viewing, detailing the function of genre scripts, and expand on their explanatory role beyond affective forecasting.

We explained our 2009 results by proposing that specifically film genre scripts contain embodied knowledge of subtle stylistic cues as perceptual simulators. An embodied format seems quite suitable for the representation of rather low-level perceptual features involved in subtle stylistic variations. Embodied simulation responses to subtle stylistic features involved can be directly associated with negative emotions. For example, viewers simulate the impact of fast actor movement associated with fright or an anticipation of pain. Lower-level perception of subtle stylistic features is effective in the vivid simulation of bottom-up driven emotional appraisal, action readiness, and experience. We have more recently presented empirical support for the possibility that genre scripts can have a generative dimension; that is, they can be used in generating genres at least when the usual requirements of film production are alleviated. We showed that subtle stylistic cues can be successfully manipulated by regular film viewers in a genre production task (Visch \& Tan 2015). Participants were presented with a brief animated film clip running in a loop. They could alter actor movements by adjusting sliders for each of four parameters of movement and were asked to set the parameters so as to create the film genres action, drama, comedy, and nonfiction. The criterion of success was a match between their settings and those obtained from professional animation filmmakers. We explained the results by assuming that regular film viewers used available knowledge of genres in a production task that was quite novel to them. The knowledge concerned consisted of perceptual simulators of actor movement (Barsalou 2008) acquired in perceptual learning during regular film viewing. We proposed that this knowledge of subtle stylistics is, in normal viewing, used to recognize a film's genre and enjoy it accordingly, but also to synthesize the vivid embodied experience of portrayed action. In our 2009 study, we found indications that the stylistic cues for fiction genres are in fact transformed versions of nonfiction representations, leading to the hypothesis that genres have their specific ways to restructure appraisals associated with negative recipient emotions. Thus, the perceptual simulators act as low-cognitivelevel decoupling or "quarantining" (Leslie 1987) devices.

Besides lower-level perceptual knowledge, genre scripts must be assumed to entail higher-order knowledge. Perceptual simulators can be linked to conceptual knowledge; they are part of what Barsalou (2003) termed perceptual symbol systems. The perceptual symbol systems entail genre labels that we demonstrated are used in categorization, but also other genre knowledge potentially as complex as regular film viewers must be assumed to avail of, in view of their using genre publicity for the selection and enjoyment of films. On that view genre scripts include conventional plots and characteristic views of the represented world, such as tragic, comic, and melodramatic. We follow Menninghaus et al. in that such script elements enable affective forecasting and, more generally, anticipations and expectancies to be generated right from the beginning of a viewing episode. These, in turn, may prime or guide top-down attention to details of image and sound that matter for generic emotions, including negative ones. The delivery on expectations makes for enjoyment of these.

To this explanation of the enjoyment of negative genre-specific emotions, we add that genre scripts may be sufficiently complex to serve pragmatic functions the sophistication of which would surpass cuing anticipations of upcoming emotions. We, in particular, forward the possibility that they enable an understanding of the filmmaker's planning of the film's narrative discourse as it develops and even offer glimpses of a master plan underlying the narration. (By filmmaker, we refer to a generalized agent perceived in charge of the narration rather than a specific person. See Bordwell's [1985] concept of a film viewer constructed "narrative intelligence.") In such a plan, the invocation of negative emotions can be perceived to have a function serving more encompassing intentions, notably the presentation of an interesting view of the represented story world and its events, characters, and themes. For example, my genre knowledge includes an intuition that my negative emotion in viewing a poignant and sad drama has a function in the filmmaker's plan to make me share a tragic view of some fictional world, with possible ramifications for my views of the real world. We propose then that genre scripts have a pragmatic dimension functional in enjoying and appreciating genre films, including but not limited to their typical unpleasant emotions. To the degree that the scripts are shared among filmmakers and film viewers - see our 2015 experiment above - they enable viewers to synthesize the perceptions and experience the filmmaker has in mind, and more generally distill what they are up to.

Thus, we propose, genre scripts may link aesthetic experiences with the perception and possible sharing of intentions with an author. We find such a communicative factor missing in the Distancing-Embracing model of the enjoyment of negative emotion in art reception. First, on a most general note, we argue that the experience of extraordinarily intense and especially negative emotions can induce needs to attribute these to particular aesthetic motivations, and if these cannot be readily identified, attribution to a maker or author may be sought. This has been found in research of anger- and disgust-inducing works of art (Silvia and Brown 2007). Second, a communicative factor might integrate the two components of the model, as both Distancing and Embracing can be means for as well as ends of sharing efforts with the filmmaker. Moreover, the feeling of sharing may in itself be rewarding, perhaps that of negative emotions and less pleasant views of the world in the first place (Embracing). Finally, the filmmaker's perceived efforts to "quarantine" negative affect may be reciprocated by a recipient's willingness to transform negative emotional appraisals (Distancing).

\section{Tuning in to art: A predictive processing account of negative emotion in art}

\section{doi:10.1017/S0140525X17001868, e377}

\section{Sander Van de Cruys, ${ }^{a}$ Rebecca Chamberlain, ${ }^{a, b}$ and Johan Wagemans $^{a}$}

${ }^{a}$ Brain and Cognition Unit, Faculty of Psychology and Educational Sciences, KU Leuven, Leuven BE-3000, Belgium; ' Department of Psychology,

Goldsmiths, University of London, London SE14 6NW, United Kingdom.

sander.vandecruys@kuleuven.be

r.chamberlain@gold.ac.uk

www.sandervandecruys.be

www.gestaltrevision.be johan.wagemans@kuleuven.be

. 
Abstract: We use the example of art-derived solace to discuss a broader mechanism by which negative affect is instrumental in creating positive appreciation of artworks. Based on the theory of predictive processing, we argue that increasing attunement or reduction of prediction errors, which implies increasing validation of the agents (models), is experienced as positive, even if the artwork's content is negative.

We commend Menninghaus et al. for their thorough and insightful treatment of negative affect in aesthetic experience. As they illustrate, negative affect is ubiquitous and nonaccidental in art, so the question of why people seek out negative affect in art is a fascinating and important one. Although Menninghaus et al. highlight several possible explanations, we think they leave underexposed the central role of consolation in art appreciation.

Very often, people read novels to find solace concerning everyday or existential uncertainties and anxieties. They watch films and television series to remind themselves they are not alone in their failings, inner conflicts, or even idiosyncratic pleasures. Even if no concrete solutions for sorrows are offered in the artwork, the mere discovery of similar affective dynamics validates the existence of the perceivers and the cognitive/affective schemata with which they experience (and navigate through) the world. Although the content of these dynamics is often negative, the process is one of increasing attunement between artwork and perceiver.

On the face of it, this could be considered a kind of empathic affect (Jackendoff \& Lerdahl 2006) where the perceiver empathizes with the creator of the artwork or the performer of the dance or music act. But the point is not that we have compassion with themes or people in the artwork and subsequently, as a kind of meta-emotion, feel good about this prosocial response (what Menninghaus et al. do discuss but dismiss). More often, the "empathic" reflection is one of more direct attunement with the artwork, for example, with the structural aspects of the music that convey sadness, joy, and so forth, by way of modulation of tone, tempo, timbre, melodic contour, and so forth.

The "language" by which the musician or painter's technique allows us to "empathize" with the work directly is insufficiently understood, but probably relates to natural expressive speech or expressive behavior (Freedberg \& Gallese 2007; Jackendoff \& Lerdahl 2006). What is clear is that we have implicit generative models of how particular artistic outputs are created (as for any other perceptual inputs [e.g., Kersten et al. 2004]). Perceiving those outputs is inferring their hidden causes, which includes not only specific patterns of motor behavior (e.g., strumming a guitar, "stroking" a canvas), but also the (hierarchically) deeper causes, in terms of conceptual and emotional/motivational states. Meanwhile, the content of the artwork can set the context for a better alignment of the models of the perceiver and those implied in the work. The commonality in generative models that allows for synchronization of state dynamics has recently been used to study mutual understanding in social interactions, where a shared conceptual space enables agents to predict each other (Friston \& Frith 2015). However, the analysis here suggests this form of alignment is not limited to explicit social interactions.

The exceptional thing about art and music is that they often invoke affective narratives that are rarely explicitly articulated, but nonetheless find resonance in the affective models of perceivers. Here, art has the potential to resolve inner conflicts or ambiguities through attunement, by confirming models that allow seemingly contradicting states to coexist or by validating one model over the other.

But good artworks will also (temporarily) obstruct alignment, and this obstruction is often conducive to appreciation. To understand this, we can turn to predictive processing as an account that formalizes the mental mechanics of uncertainty reduction. This approach holds that our brain is continually attempting to minimize the prediction errors that reflect the mismatch between its hierarchical models (that we use to predict/interpret inputs) and the actual inputs from our senses (Clark 2013; Friston 2010).
We previously hypothesized that the transition from a state of higher uncertainty to a state of lower uncertainty (i.e., an active reduction of prediction errors) is experienced as pleasurable, and that this may help explain our aesthetic experiences, even for "static" artworks (Van de Cruys \& Wagemans 2011). Hence, the increasing attunement of models and world (perceptual inputs) yields positive affect (again, even if the content of the models/inputs is about negative events). So, with respect to emotional effects, the dynamics of attunement are key. If violations of expectancies are the norm in art, this is because it allows viewers to make greater progress in reducing those "errors" on conceptual or perceptual levels.

If we extend the view above and characterize negative affect as a state of rapidly increasing uncertainty (i.e., the opposite of positive affect), then negative affect is indeed regularly present in our experience with art - in a formal way, not only in terms of negative content. The question of why we seek out negative affect in art then becomes very closely related to the much debated one about why we sometimes seek out uncertainty. One way out, within a predictive processing framework, is to argue that we are intrinsically motivated to seek predictive progress (Gottlieb et al. 2013), which necessarily urges us to venture out of predictable zones. However, we do not do this haphazardly; we seem to contextually learn (meta)predictions on prediction error reduction rates (Van de Cruys 2017). Those expectations on the reducibility of errors can function as implicit appraisals of coping potential that are so important in our engagement with art (Silvia 2005b).

In sum, although we started out describing the important role of solace in art, we came to see it as just an example of broader mechanism by which negative affect is instrumental in creating positive appreciation of artworks. The presented account may serve as an explanation for the "mood congruency effect" (Derryberry 1988), in which perceivers seek out artworks that correspond to their current or desired mood state (e.g., sad music on a funeral). The view also has some affinity with the processing fluency account, but it adds a process account and emphasizes the temporal dynamics in fluency (cf. relative fluency [Wänke \& Hansen 2015]). We end up with a view in which "being moved by" art is an almost literal consequence of moving through the prediction error gradients.

\section{Explaining the enjoyment of negative emotions evoked by the arts: The need to consider empathy and other underlying mechanisms of emotion induction}

\author{
doi:10.1017/S0140525X1700187X, e378
}

\section{Jonna K. Vuoskoski ${ }^{\mathrm{a}, \mathrm{b}}$ and Tuomas Eerola ${ }^{\mathrm{b}, \mathrm{c}}$ \\ ${ }^{a}$ Faculty of Music, University of Oxford, Oxford OX1 1DB, United Kingdom; ${ }^{b}$ Department of Music, Art and Culture Studies, University of Jyväskylä, Jyväskylä 40014, Finland; ${ }^{c}$ Department of Music, Durham University, Durham DH1 3RL, United Kingdom. \\ jonna.vuoskoski@music.ox.ac.uk tuomas.eerola@durham.ac.uk http://users.ox.ac.uk/ musf0093/ \\ https://community.dur.ac.uk/tuomas.eerola/}

Abstract: Any model aiming to explain the enjoyment of negative emotions in the context of the arts should consider how works of art are able to induce emotional responses in the first place. For instance, research on empathy and the arts suggests that the psychological processes that mediate the enjoyment of sadness and horror may be fundamentally different.

The Distancing-Embracing model outlined by Menninghaus et al. is an impressive and comprehensive account of how negative emotions can intensify engagement and enjoyment in the context of 
the arts. Although we applaud the broad, integrative approach adopted by the authors, and recognize the theoretical and practical value of their model, we comment here upon certain limitations and potential inconsistencies that, in our opinion, may hinder the formulation of theory-derived predictions and hypotheses for empirical testing.

Although we agree that personal safety, control, and intentionality (i.e., the notion that emotions experienced in art contexts are typically self-sought) are indeed crucial preconditions for the enjoyment of negative emotions in the context of the arts, we find the overarching concept of "psychological distance" internally contradictory. Although the lack of direct personal goal relevance typically associated with art-elicited emotions could indeed be construed as "psychological distance," the other preconditions control and intentionality - are less compatible with "distancing"; in fact, they imply voluntary proximity and engagement and facilitate greater immersion, identification, and transportation. Empirical evidence has shown that greater immersion (and intensity of negative emotion) is positively associated with the enjoyment of narratives and music (e.g., Eerola et al. 2016; Green et al. 2004; Sparks 1991; Tal-Or \& Cohen 2010), and it could even be argued (as hinted at by the authors themselves) that transportation and the temporary suspension of disbelief may be conducive - or even a prerequisite - to enjoyable engagement with narrative fiction and other arts (cf. Green et al. 2004). Because of these positive associations between immersion, intensity of negative emotions, and enjoyment, we argue that the labelling of the Distancing factor is potentially misleading.

In our view, the most crucial limitation in the multicomponent model proposed by Menninghaus et al. is the omission of psychological mechanisms involved in the induction of emotion in the context of the arts. We argue that any model aiming to explain the enjoyment of negative emotions in the context of the arts should consider how works of art are able to induce emotional responses in the first place. Although the authors discuss empathy briefly in terms of a "meta-emotion," where sadness gets transformed into pleasure through a self-gratifying prosocial response, we argue that empathy and empathy-related processes (embodiment, theory of mind, emotional contagion, and simulation) are actually fundamental to our engagement with (and production of) various forms of art, enabling emotion induction, transportation/immersion, and sense making. In the context of music, for example, empathic processes are thought to take place on multiple levels, ranging from preconscious, internal mimicry of emotionally expressive acoustic and gestural cues (e. g., Molnar-Szakacs \& Overy 2006) to imaginative perspective taking and mentalizing evoked by the process of music listening (e.g., Clarke et al. 2015; Levinson 2006) and extra-musical information (Vuoskoski \& Eerola 2015). These processes may be fundamental in understanding why we find engaging with arts and fiction enjoyable - including engagement with negative emotions, as empirical studies have associated trait empathy and deliberate perspective taking with greater intensity (even at the level of psychophysiology) and greater enjoyment of negative emotion in the context of music, opera, and film (e.g., Eerola et al. 2016; Miu \& Baltes 2012; Vuoskoski et al., 2012; Zickfeld et al. 2017). However, althoughempathy and related processes may play an important role in facilitating feelings of being moved and the enjoyment of sadness-evoking works of art (e.g., Eerola et al. 2016; Vuoskoski \& Eerola 2017; Wassiliwizky et al. 2015; Zickfeld et al. 2017), this is probably not the case for horror films and suspension, for example. In fact, empirical evidence reveals a negative relationship between empathy and the enjoyment of horror films (Tamborini et al. 1990). These divergent relationships between empathy and the enjoyment of sadness and fear/horror in the context of arts highlight the need to consider the psychological mechanisms of emotion induction in association with the enjoyment of those emotions. For instance, although feelings of being moved and suspension may indeed both be mixed emotions that mediate the enjoyment of sadness and horror (respectively), the psychological processes through which this mediation takes place may be fundamentally different in the two cases.

Finally, we address the somewhat confusing use of the term transformation. Menninghaus et al. firmly reject the notion of "a full-blown transformation of negative into positive affect" (sect. 4.6), while simultaneously characterizing their model as a "twofactor transformation model." Despite this characterization, it is not explicated what kind of (partial?) transformation takes place and where, and thus, it is unclear whether the notion of "transformation" is an accurate (or necessary) characterisation of the processes involved. However, if the model actually rejects transformation, it would be constructive to label the building blocks differently.

Despite the aforementioned limitations and points of potential confusion, the Distancing-Embracing model undeniably moves the field closer to understanding the wide appeal of negative topics in the arts. Future work should strive to investigate the psychological processes underlying the different components outlined in the broad model and to put forward a more detailed account of how different mechanisms of emotion induction contribute to - and interact with-the model components. To put this plea more broadly, it would be important to specify the ontological bases of the different model components, as the assumptions concerning the interpretation of mixed emotions and cultural scripts (for instance) depend largely on the framework in which they are construed.

\section{Embracing nonfiction: How to extend the Distancing-Embracing model}

\author{
doi:10.1017/S0140525X17001881, e379
}

\section{Deena Skolnick Weisberg ${ }^{\mathrm{a}}$ and Stacie Friend ${ }^{\mathrm{b}}$ \\ ${ }^{a}$ Department of Psychology, Levin Building, University of Pennsylvania, Philadelphia, PA 19104; ' ${ }^{\mathrm{b}}$ Department of Philosophy, Birkbeck, University of London, London WC1E 7HX, United Kingdom. \\ deena.weisberg@psych.upenn.eduｓ.friend@bbk.ac.uk https://www.sas.upenn.edu/ deenas/ http://www.bbk.ac.uk/philosophy/our-staff/academic_staff/dr-stacie- friend}

Abstract: The Distancing factor of Menninghaus et al.'s model includes schemas that remind consumers that the representation is fictional. Although they claim that these schemas are crucial to the functioning of the Embracing factor of the model, we argue that consumers can have similar responses to nonfictional representations. We urge the authors to expand their model to include such cases.

Menninghaus et al. propose a model of why and how the enjoyment of negative emotions is a crucial feature of art reception. The most important aspect of this model, for our purposes, is its emphasis on psychological distance. Within the Distancing factor of their model, they highlight three cognitive schemas that serve to distance the consumer from the artwork: art, representation, and fiction. Together, these schemas remind consumers that what they are experiencing is not real, allowing the Embracing components of the model to function.

We do not object to the claims that distance is required for many types of aesthetic appreciation and that one can embrace negative emotions in the context of such appreciation. However, although the authors do not claim to provide necessary and sufficient conditions for distancing, they do imply that the art and especially fiction schemas are primarily responsible for this effect in narrative representations. We believe that the inclusion of these schemas leads the authors to apply their model too narrowly. We suggest that the art and fiction schemas provide nothing more than optional mechanisms to achieve the distance necessary for embracing. They may fail to function as described, and other mechanisms may function equally well. 
Response/Menninghaus et al.: The Distancing-Embracing model of the enjoyment of negative emotions in art reception

One the one hand, fictional artworks may fail to trigger sufficient distance to allow for the enjoyment of negative emotions. For example, a mother who has suffered a miscarriage or a soldier who has experienced combat would likely not enjoy the negative emotions provoked by a fictional story about a miscarriage or a battle. In the famous discussion of "psychical distance" cited by the authors, Edward Bullough points out that a jealous husband may not be able to appreciate Othello. These cases have the features required by the Distancing part of the model - they are fictional, artistic representations - and yet do not create distance as the authors suggest they should. In such cases, the negative emotions overwhelm and prevent aesthetic enjoyment.

On the other hand, and more interestingly, the majority of the features in the Embracing factor of the model can (and often do) apply to nonfictional and even non-art representations. For example, journalistic photographs from the Great Depression or a narrative biography can easily elicit an interplay of emotions or spark a search for meaning or higher-order symbolism in the same way as fictional pieces do. In these cases, a consumer is aware that real harm was done and that real people suffered; the art and fiction schemas are not activated. Nevertheless, in these cases, we can experience the heightened aesthetic enjoyment generated by Embracing - arguably because these cases are suitably distant even though they are not fictional.

Perhaps most tellingly, in this same vein, consumers may not know whether a given representation is fictional, or may be unsure of its status. For example, one might experience and enjoy negative emotions elicited by a magazine article about a family tragedy despite not knowing whether it is a short story or a piece of narrative journalism. Cases like this provide strong reasons to reject the authors' claim that the Distancing schemas must be activated in advance ("situational activation of these schemata precedes the online processing," sect. 1, point B, para. 1), because this cannot be true in cases where one does not know if one if viewing a fictional or nonfictional representation. This is not to deny that knowledge of a representation's fictionality plays a role in our responses, but it is to deny that we experience the enjoyment of negative affect only in representations already framed as art or fiction.

We thus would encourage the authors to modify the Distancing factor of their model to make clear that the fiction and art schemas are no more than possible mechanisms for generating psychological distance, rather than standard requirements. If they do so, we believe that their model will apply to a broader set of cases of aesthetic appreciation and improve our understanding of the role of negative emotions in the enjoyment of a wider range of representations.

In fairness, the authors do seem to consider this option at several points in their article. For example, they write, "There are even clear cases of fascination when viewing live footage of real disastrous events.... [W] here no distancing effects of fiction and temporal distance and real humans are involved, some spatial distance - sufficient enough to support the precondition of the viewer's personal safety-is still required" (sect. 3.3, para. 2). We agree, but we believe that this simple acknowledgment that such cases exist does not go far enough. Indeed, the authors seem actively resistant to the kind of broadening we suggest. In their article (sect. 4.2.1, last para.), they state that there are "subtle differences in emotional responses that are still likely to derive from the ontological distinction between real represented and fictional represented events."

This resistance to including nonfictional cases of the embracing of negative emotions thus seems to depend on the assertion that the emotions engendered by these cases will be significantly different from the emotions engendered by fiction. But even if these emotions are different in some respects, surely they share enough commonalities to serve the functions required by the Embracing factor of the model. Further, this is an empirical question. In the absence of firm evidence for it, we believe that the similarities between fictional and nonfictional cases should be sufficient to apply the authors' model to both. Doing so would provide a framework for a more general theory of our enjoyment of negative emotions in representations.

\section{Authors' Response}

\section{Negative emotions in art reception: Refining theoretical assumptions and adding variables to the Distancing-Embracing model}

\author{
doi:10.1017/S0140525X17001947, e380
}

\begin{abstract}
Winfried Menninghaus, ${ }^{\text {a }}$ Valentin Wagner, ${ }^{\text {a Julian Hanich, }}{ }^{\text {b }}$ Eugen Wassiliwizky, ${ }^{\mathrm{a}}$ Thomas Jacobsen, ${ }^{\mathrm{c}}$ and Stefan Koelsch $^{\mathrm{d}}$

${ }^{a}$ Department of Language and Literature, Max Planck Institute for Empirical Aesthetics, 60322 Frankfurt am Main, Germany; ${ }^{\mathrm{b}}$ Department of Arts, Culture and Media, University of Groningen, 9700 AB Groningen, The Netherlands; ${ }^{c}$ Experimental Psychology Unit, Helmut Schmidt University/University of the Federal Armed Forces Hamburg, 22043 Hamburg, Germany; ${ }^{\text {dUniversity of }}$ Bergen, 5020 Bergen, Norway.

w.m@aesthetics.mpg.de

valentin.wagner@aesthetics.mpg.de

j.hanich@rug.nl
\end{abstract}

eugen.wassiliwizky@aesthetics.mpg.de

jacobsen@hsu-hh.de

stefan.koelsch@uib.no

https://www.aesthetics.mpg.de/en/the-institute/people/prof-dr-winfried-

menninghaus.html

https://www.aesthetics.mpg.de/en/the-institute/people/dr-valentin-

wagner.html

http://www.rug.nl/staff/j.hanich/

https://www.aesthetics.mpg.de/en/the-institute/people/e-wassiliwizky.

html

https://www.hsu-hh.de/allgpsychologie/index_FPDCF8Hp1Z8V4KmF.

html

http://www.uib.no/en/persons/Stefan.K\%C3\%B6lsch

Abstract: While covering all commentaries, our response specifically focuses on the following issues: How can the hypothesis of emotional distancing (qua art framing) be compatible with stipulating high levels of felt negative emotions in art reception? Which concept of altogether pleasurable mixed emotions does our model involve? Can mechanisms of predictive coding, social sharing, and immersion enhance the power of our model?

\section{R1. Introduction}

We are very grateful for the time and the intellectual acumen so many respondents devoted to our article. The commentaries come from an astounding variety of fields and perspectives. We take this to imply that the topic under consideration is, indeed, very rich in implications.

In the first section of our response, we exclusively discuss commentaries that directly address the components of our model; we do this in the order in which these components appear in our article. In the second section, we respond to commentaries that discuss issues for which our model includes explicit provisions, but which were left untreated. The third section addresses commentaries proposing additional or alternative variables regarding the enjoyment of negative emotions for which our model does not include any explicit provision.

Throughout our response we place a primary focus on discussing controversial issues. This specifically concerns the seemingly paradoxical hypothesis that the distancing implications of an art framing need not reduce the felt intensity of negative emotions to make them compatible 
with enjoyment, and our hypothesis of being moved as a positive emotion with a mixed affective signature. Regarding additional variables, mechanisms of predictive coding, social sharing, and immersion are discussed in greater detail than other aspects, because they were brought up in several commentaries.

To begin, we respond to two commentaries regarding the interdisciplinary design and the scope of our article. Our model clearly makes an effort to integrate humanist traditions of theorizing the enjoyment of negative emotions and recent scientific theories and empirical findings. We have not, however, aimed at unifying "holistic" ("Romanticist") and "atomistic" approaches, as suggested by Cupchik. The tradition of rhetoric and poetics we draw on is (mostly) not of a "holistic" and Romanticist nature, nor are the scientific theories and findings we refer to inherently "atomistic."

Second, our model proposes a new, integrative perspective on existing theorizing and available empirical data, but it does not provide any new data. To our knowledge, neuroscientific research on coactivations of negative emotions and feelings of reward is only beginning, and several pertinent studies (Brattico et al. 2016; Ishizu \& Zeki 2017; Wassiliwizky et al. 2017b; see also the commentary by Brattico \& Vuust) were only published after the acceptance of our article. As a result, we cannot but agree with the comment by Nadal, Vartanian, \& Skov (Nadal et al.) that our model does not provide a "neurobiological basis" for the topic under consideration. At the same time, we strongly disagree with the claim that a psychological model that does not specify the neural circuitry underlying its hypothetical mechanisms cannot provide useful guidance for future empirical research. (If this were true, all psychological models proposed prior to the advent of neuroscience would have been of little, if any, use for empirical research.)

\section{R2. Commentaries addressing the individual components of our model}

\section{R2.1. Negative emotions are a predestined resource for the arts (= Hypothesis 1)}

In our introductory section, we juxtapose two findings from two distant traditions. The first finding (A) comes from recent psychological research: In comparison to positive emotions, negative emotions have empirically been shown to prioritize attention, to have a distinct potential for a high intensity of subjective feeling, and to enjoy privileged storage in memory. The second finding (B) dates back to ancient rhetoric and poetics: The relevant treatises converge in suggesting that the powers to prioritize attention, to support strong emotional involvement, and to secure access to memory are precisely what the arts in general strive for. We conjecture (= Hypothesis 1$)$ : if $\mathrm{A}$ and $\mathrm{B}$, then it might be concluded that $(\mathrm{C})$ negative emotions are actually predestined resources for the arts rather than exceptional liabilities.

Reconsidering the literature, one could challenge the notion that (A) and/or (B) are correct summaries of findings/theories in the two research fields; in this case, (C) would not follow. One could likewise try to put forward arguments as to why $(\mathrm{C})$ would not necessarily follow, even if $(\mathrm{A})$ and (B) are right. Our conjecture was not challenged on either of these grounds. It was, however, challenged for reasons that misrepresent our argument. Nadal et al. (para. 3) write: "This assumption is clearly untenable, because not all interactions with art involve negative emotions" (Martindale 2001; Smith 2014). We note that we only propose that negative emotions may be predestined resources for the arts' efforts and not that they are mandatory resources. Moreover, we explicitly stress: "we do by no means rule out that visual representations of beautiful humans, animals, landscapes, stills, and so forth can well be enjoyed as beautiful without necessarily co-evoking any negative emotional associations" (sect. 1, final paragraph).

Regarding another conceptual issue, we fully subscribe to the remark by Vuoskoski \& Eerola that our model should not be described as a "transformation" model. After all, the activation of an art framing typically precedes the actual processing of artworks. Accordingly, we do not imply-as suggested by Leder \& Schwarz - that an undistanced negative emotion is first experienced and only post hoc relieved from some important aversive implications associated with negative emotions in an ordinary reality framing. Our model likewise does not predict that the Embracing components "transform" the negative emotions into positive ones, but rather that they enhance their compatibility-as negative emotions - with enjoyment. For these reasons, at some stage of writing the article, we systematically erased the term transformation. It has, however, survived this revision in three sentences. We are grateful for the opportunity to clarify this inconsistency.

\section{R2.2. Elicitors of negative emotions play a great role in artworks, and the corresponding emotions are indeed experienced (= Hypothesis 2)}

As reported in our article, several 20th-century philosophers have advocated the notion that art recipients may actually not experience any relevant levels of genuine negative emotions, but only erroneously misattribute negative emotions to their felt responses. To rule out this possibility, we reviewed available empirical findings regarding both self-reported subjectively felt emotions and objective measures of physiological indicators of such emotions. This review provided substantial evidence for Hypothesis 2 as stated above.

The commentary by Konečni is the only one that challenges this hypothesis. On the one hand, Konečni elaborates on differences between art-elicited emotions and emotions in more ordinary life-contexts. These differences are by no means disregarded by our model but are in fact the very core of what we call the Distancing effects resulting from the activation of the situation schema of art reception. On the other hand, referring in a very general way to well-known difficulties of interpreting changes in physiological and electromyographic (EMG) data as indicative of genuine emotional episodes, Konečni dismisses all reported physiological evidence in a wholesale fashion, without discussing the respective studies in any detail. Therefore, his remarks are far from providing a scientifically sound refutation of all evidence in favor of the experiencing of negative emotions in art reception. (For new evidence regarding the actual experiencing of negative emotions in art reception, see also our comments regarding section $4.2[$ [= Hypothesis 5].) 
Response/Menninghaus et al.: The Distancing-Embracing model of the enjoyment of negative emotions in art reception

\section{R2.3. The Distancing factor of our model}

Top-down cognitive influences on processing negative emotions constitute the Distancing factor of our model. Our review of the pertinent literature can be summarized as follows: Negative emotions experienced in art contexts differ from ordinary emotions by virtue of being cognitively framed as art-, representation- and/or fiction-elicited emotions. Because these framings imply personal safety and control over continuing or discontinuing the exposure, they keep some of the aversive implications that negative emotions would typically have in real life contexts at a psychological distance. As a result, the art framing has the potential to allow for higher beauty, liking, and other positive ratings compared to a reality framing, even without reducing the levels of the felt negative emotions involved (= Hypothesis 3). This latter qualification is crucial, because if the art framing would reduce the intensity of felt negative emotions, the Distancing component of our model would be incompatible both with our Hypothesis 1 , according to which negative emotions are valuable resources for the arts (rather than mere antidotes to be tamed or converted), and with our Hypothesis 2, stipulating that negative emotions are actually experienced to significant degrees during art reception.

A remark by Nadal et al. suggests a mismatch between our model and the evidence we rely on: "The DEM [Distancing-Embracing model] predicts that these schemata [i.e., the art, representation, and fiction schemata] should influence the experience of negative emotions. However, Wagner et al. (2014) and Gerger et al. (2014) - cited as supporting studies - found no such influence." We disagree. The two studies do show what our model predicts, namely, that art framings, compared with reality framings, render different sets of pictures with affectively negative content more aesthetically enjoyable, without reducing, let alone erasing, the levels of felt negative emotions. In other words, they reveal that because of an art framing, substantial levels of negative emotions are compatible with, if not conducive to, higher aesthetic liking.

To be sure, both philosophers and appraisal theorists have predicted precisely what Nadal et al. apparently mistook for our position: that in the absence of challenges to own personal goals, art-elicited emotions, and most notably negative ones, should have a lower intensity in terms of subjective feeling, motor expression, and peripheral physiological measures when compared with emotional responses to analogous stimuli in real-life contexts (cf. Frijda 1988, p. 352; Lange 1901, pp. 100-05; Lazarus 1991; Martindale 1984; Scherer 2005). We do not question this view for real-life contexts. However, several researchers, including emotion psychologists, have proposed a divergent perspective for art contexts. They hypothesized, and could partly show, that the suspension of prototypical action responses that results from the absence of challenges to own safety and other important goals makes room for a higher second-order awareness of one's felt sensations (Lambie \& Marcel 2002) and may actually support an increased intensity of felt emotions (Gross \& Levenson 1997; Maslow et al. 1970; Oatley 1994; Tan 2000, p. 117; Visch et al. 2010). The appraisal theorist Nico Frijda pushed this reasoning even to the point of claiming that art recipients intensely and hedonically "indulge" in these pragmatically useless emotions for their own sake (Frijda \& Sundararajan 2007).
Notably, the theoretical assumptions referred to above did not specifically consider the role of negative emotions. This is where our model makes a difference. In general, it is typologically similar to the above-referred-to perspectives that give the absence of challenges to own goals, safety and action responses a decisively different turn for art-elicited, compared with garden variety, emotions. At the same time, following Batteux's remark that an art context is more beneficial to negative than to positive emotions, our model predicts differential benefits of the art framing for negative compared with positive emotions (for all details, see sect. 3 of our article). And it moreover spells out five art-specific mechanisms that support a Distancing-enabled Embracing specifically of negative emotions. As suggested in the commentaries by Armstrong \& Cutting and Vuoskoski \& Eerola, immersion and transportation are likely to be further processes in art reception that counteract the tendency for reduced intensity of felt negative emotions predicted by a classical appraisal perspective.

The hypothesis of an enjoyable high intensity of felt negative emotions is clearly supported by the empirical evidence provided in our article and, even more strikingly, by the additional recent evidence we report in our response concerning commentaries on section 4.2. Hogan's commentary even suggests that nonaversive exposure to simulations of aversive events may be adaptive for coping with similar challenges in real-life contexts.

Most commentaries do not challenge our hypothesis of Distancing, but focus on refining the predictions of our model and including more provisions for cases in which it may not work as predicted. Gerger, Ishizu, \& Pelowski (Gerger et al.) suggest that the effects of the distancing mechanisms may not be a binary issue of being on versus off, but may vary over the temporal course of aesthetic trajectories. Moreover, there may be numerous gradations, depending on how unambiguous or ambiguous the framing is perceived to be (Weisberg \& Friend), how representational or nonrepresentational the artwork is, and the extent to which it involves further dimensions of indeterminacy $(\mathbf{R a b b})$. Live stage performances of individuals in pain and distress may have so immediate and visceral an impact on viewers that they reduce the art framing effect (Goldstein). Moreover, the adoption of an "aesthetic stance" or "focus" may yield effects similar to those of an art framing (Brattico \& Vuust). We consider all of these remarks as potentially adding modifying variables, and hence higher granularity of predictive power, to our Distancing factor.

Finally, Davies deplores that our discussion of Distancing does not cover several contributions from analytic philosophy in the 1960s. We note first that our article has an expressed bias toward reviewing theoretical hypotheses in the light of empirical evidence. Second, because we consider the Embracing factor to be the more original part of our model, we deliberately spent only roughly oneseventh of our main text on the Distancing factor. Within these constraints, we could not cover the rich philosophical discussion in a more inclusive way.

\section{R2.4. The Embracing factor}

The longest section of our article - and the one on which we place the strongest focus - is devoted to the Embracing 
factor. Compared with the other sections and their corresponding hypotheses, this section has far fewer antecedents in the existing literature. The five subsections propose five mechanisms that allow art recipients not only to tolerate, but even to "embrace" negative emotions.

R2.4.1. Interplays of positive and negative emotions are more enjoyable than purely positive emotional trajectories, because they involve more dynamic change and emotional variety and are therefore more engaging, livelier, richer, and less likely to elicit boredom and lack of interest (= Hypothesis 4$)$

Section 4.1 draws on hypotheses advocated in both poetics and philosophical aesthetics. The hypothesis is well in line with basic principles of both philosophical and empirical aesthetics. Moreover, two articles on visual design that we came across only very recently (Fokkinga \& Desmet 2012 ; 2013) make a similar case for a design-driven inclusion of negative emotions in consumers' interactions with consumer objects.

In his commentary, Oatley refers to two interview studies (Djikic et al. 2006; 2009) that he presents as (implicitly) supporting our hypotheses. In a nutshell, the studies show that when speaking about their own work, writers use more negative emotion words than members of a control group when speaking about their respective professional activity, and that readers show greater changes across a broad range of emotions, including negative emotions, after reading a genuine literary narrative by Chekhov compared with readers of a control version having the same content, length, and reading difficulty.

Regarding the visual arts, Chatterjee suggests that paintings, photographs, and other images can encapsulate and condense time into "peak moments" (that reach out into the past and future) or "distilled moments" (that primarily reach out only into the past) and that such compositional techniques support a dilation of time perception that enables viewers to experience analogues to the temporal interplays of positive and negative emotions that our hypothesis stipulates primarily for works of literature and music. Ainslie's commentary points out that the enjoyment of negative emotions as stipulated by our model is likely to apply far beyond the confines of art, and sketches a rich picture of how and why this might be the case.

Summing up, Hypothesis 4 raised no critical concerns, but exclusively received commentaries that lend it additional support.

R2.4.2. Concomitant mixed emotions serve as mediators of negative emotions' positive contributions to enjoyment (= Hypothesis 5)

Section 4.2 gives the hypothesis discussed in section 4.1 a more specific turn. Rather than merely arguing for emotional variation, it attributes a crucial role to mixed emotions. Among the three examples we discuss (being moved, suspense, and disgust), the case of being moved received the most detailed and the most critical comments. We therefore focus on this example in our response.

R2.4.2.1. Are there actually episodes of being sadly moved? And how can being moved be both a positive and a mixed emotion? Schubert Seibt, Zickfeld, Blomster, \& Fiske (Schubert et al.). suggest that being moved is a positive emotion primarily associated with happiness and that the close association of being moved with sadness that we propose is a misunderstanding. We fully agree that being moved is, overall, a positive emotion. At the same time, we also maintain our view that sadness is a key emotional ingredient of the complex emotion of being moved. The existing literature clearly supports our view. In a free association task that asked participants to list emotions that they closely associate with the concept of being moved (Kuehnast et al. 2014), the combined listings of sadness (German Traurigkeit) and mournfulness (German Trauer) ranked highest; joy and happiness were next. Based on Japanese, Finnish, and German participant pools, six other studies reported converging results, including studies that experimentally induced states of being moved (Hanich et al. 2014; Menninghaus et al. 2015b; 2017; Tokaji 2003; Vuoskoski \& Eerola 2017; Wassiliwizky et al. 2015). All of these studies provide evidence for two prototypes of being moved, one being the sadly moving type and the other the joyfully moving type. On the basis of 106 detailed verbal accounts of feeling moved and with the use of latent class analysis, a more recent qualitative study from our group (yet unpublished) arrived at the same finding.

Moreover, with the exception of Hanich et al. (2014) and Vuoskoski and Eerola (2017), who exclusively considered sad films and sad music and, thus, did not address joyful episodes of being moved, the studies just mentioned also revealed that the two prototypes of being moved are by no means mere opposites. Rather, both prototypes feature blends of positive and negative ingredients, if only in inverse proportions. For example, emotionally moving narratives of reconciliation and reunion elicit strong feelings of joy and happiness, yet these positive emotions are typically blended with, and amplified through, an awareness of the painful preceding separation. This motivates our use of the term mixed emotions for episodes of being moved.

Finally, in a study on sadly moving films (Hanich et al. 2014), we provided evidence that high ratings for being moved, even if they positively correlated with high ratings for sadness, amounted to a strongly positive overall evaluation of the eliciting film clips as artworks and, hence, to a clearly and non-ambivalently positive aesthetic emotion (for the concept of "aesthetic emotions," see Schindler et al. 2017). Suggesting a distinction between "micro" and "macro" levels of emotional valence, Shuman et al. (2013) provide a non-contradictory conceptual framework for this finding. On this view, being moved can have both a mixed affective valence on the micro level of its temporal trajectory and an altogether positive affective valence on the macro level.

Schubert et al. do not discuss why we and others may have arrived at results that diverge from theirs. We suggest two potential reasons. First and foremost, Schubert et al. focus primarily on feelings of kama muta and collect ratings for being moved only as a vernacular proxy for this target construct. It is not clear, however, how they account for potential differences between the two concepts and to what extent their preconception of kama muta may have favored a selective bias in the study design such that the full range of episodes of being moved was not covered. In fact, several elements of this full range, as detailed by various methods of conceptual mapping in Kuehnast et al. (2014) and Menninghaus et al. (2015b), are not 
reflected in the kama muta-focused studies. Second, and most likely as a consequence of the prime focus on kama muta, Schubert et al. (2016) used primarily joyfully moving stimuli, such as reunions, acts of social bonding, and selfless acts of generosity, in their study.

Schubert et al. also find our concept of metonymy to be ill-defined. They obviously conflate it with the traditional rhetorical concept of metonymy. However, we expressly referred to Roman Jakobson's definition (1973) in which metonymy signifies a broad variety of (associative) contiguity relations across combinations of words (or other events); in these cases, the contiguity relations imply that the related entities inform each other's meanings. In a similar vein, feelings of being moved are routinely associated with sadness and joy (happiness), both in semantic space and in the context of emotionally moving episodes. Importantly, such associations are not necessarily grounded in similarity (which would be required in the case of metaphor, but not metonymy). Therefore, the concept of metonymical contiguity associations does not require feelings of being moved to necessarily share the valence of sadness to be closely associated with it.

Both Schubert et al. and Nadal et al. understand our hypothesis of mixed emotions as mediating emotions to imply that any sound empirical evidence should show simultaneous (online) coactivation of positive and negative affect, and they deplore the absence of such evidence. Our response is threefold. First, in our understanding of a theoretical review paper, the absence of "gold standard" evidence is not a reason to not propose a clearly testable hypothesis. Second, in the meantime we have published evidence of the type requested. Specifically, we have shown that peak moments of being moved that are induced by film clips (Wassiliwizky et al. 2017a) and poems (Wassiliwizky et al. 2017b) are associated to a greater degree with facial electromyographic activity of the corrugator (indicating negative affect) than with zygomaticus activity (indicating positive affect). Importantly, the study on poems also reports peak levels of both hemodynamic responses in the primary neural reward circuitry and corrugator activity for the same participants and the same moments of exposure to recited poetry of an emotionally moving nature. Additional time-course analyses of these data (not yet published) based on grand averages for 1595 chills episodes reveal that these episodes show not only peak activations of the primary reward circuitry and of corrugator activity, but also of zygomaticus activity. Importantly, however, the absolute levels of corrugator activity are substantially higher than those of zygomaticus activity.

These recent findings strongly support our hypothesis that, in contexts of art reception, negative emotions are not antidotes to be tamed or converted, but rather directly co-occur with concomitant reward in moments of intense emotional experiences. Notably, the studies show that intense reward-related neural activity need not go along with affectively congruent higher zygomaticus activity compared with corrugator activity (as suggested in the commentary by Leder \& Schwarz), at least not in the context of chills responses, which show peak activations of the neural reward circuitry combined with peak corrugator activity.

Third and finally, our hypothesis of metonymical contiguity questions the notion that mixed emotional episodes must always show strict online coactivations of their antithetical ingredients, let alone involve positive and negative emotional implications to near-equal degrees (i.e., the case of ambivalence). Rather, the antithetical ingredients of mixed emotions may not be fully parallel and have consistent weight during the entire time course of an emotional episode, but may oscillate, or vacillate, in a partly nonsynchronous way (Carrera \& Oceja 2007; Larsen \& Green 2013; Larsen \& McGraw 2011; Oceja \& Carrera 2009). The various time scales at which we process actions, perceptions, and predictions may facilitate the experience of "multiple emotions 'at the same time" (Hoemann et al. 2017), particularly in art contexts that favor a systematic integration of all parts of an aesthetic and emotional trajectory into a well-composed whole. In any event, the discussion triggered by our model points to open questions regarding the very concept of mixed emotions.

R2.4.2.2. Does the enjoyment of sadness in music always take a detour through being moved?.Performing path analyses, three studies on film clips and music (Hanich et al 2014; Vuoskoski \& Eerola 2017; Wassiliwizky et al. 2015) arrived at the conclusion that sadness contributes to enjoyment not directly, but only by invigorating feelings of being moved that, in turn, directly contribute to enjoyment. (As reported in our article, other studies have mentioned feelings of peace and quiet as potential mediators.) Referring to an unpublished study, Barrett, Schulkin, \& Bernacer (Barrett et al.) argue that feelings of sadness in response to music are inherently pleasant by virtue of their "high granularity" (Feldman Barrett 2004; Kantor-Martynuska \& Horabik 2015; Lindquist \& Feldman Barrett 2008; Smidt \& Suvak 2015). Given the focus of our Hypothesis 4 on emotional variety, our model may well be compatible with the hypothesis of high emotional granularity. In any event, the issue calls for further consideration and empirical testing, once the evidence for Barrett et al.'s hypothesis has been published.

R2.4.2.3. Is boredom a case for our hypothesis?.Both the tradition of aesthetics and our article deal with only a select range of negative emotions Elpidorou asks the interesting question of whether our hypothesis of mixed emotions as redeemers of negative emotions may apply to all negative emotions. Specifically, he points out that our article refers to art-elicited boredom exclusively as a potential failure to be avoided, and asks: Couldn't it be that boredom, too, becomes more of a mixed emotion by context-driven associations with positive emotions, and thus also supports some sort of pleasure in special contexts? Elpidorou himself suggests that boredom might trigger a self-regulatory search for meaningful and interesting activities. This would clearly be a positive outcome of boredom; however, it would still not turn the experience of boredom into an inherently rewarding emotional episode. We suggest that, in a certain frame of mind, boredom in the sense of the absence of interesting stimulation might also be conducive to reaching higher levels of meditation-like states (see also Kracauer 1995). In any event, Goncharov's famous novel Oblomov (1859/2009) would be a good test case regarding how readers can enjoy a consistent and lengthy representation of the boring life of a completely lazy and inactive rich protagonist. 
Response/Menninghaus et al.: The Distancing-Embracing model of the enjoyment of negative emotions in art reception

R2.4.3. Aesthetically appealing uses of the media of representation (such as sound/music, words/language, color/shape) have the power to make the processing of negative emotional content or associations more enjoyable while not reducing, let alone erasing, negative emotional responses (= Hypothesis 6$)$

This hypothesis, even though intuitively readily accessible, has informed very little research in the field of empirical aesthetics, and only the commentary by Armstrong \& Cutting directly bears on the hypothesis. They suggest that "the well-orchestrated, seamless editing strategies" adopted by many current filmmakers promote the impression of narrative cohesion and may thereby enhance states of narrative immersion and transportation. As these states have in turn been shown to be conducive to enjoyment, the formal editing strategies bearing on low-level perceptual mechanisms might also contribute to rendering the exposure to aversive events more enjoyable. If demonstrated empirically, this testable assumption would clearly constitute a piece of evidence supporting our Hypothesis 6 .

R2.4.4. Interpretive efforts towards meaning making contribute to (re)appraising negative emotional content and concomitant feelings in a (more) positive and enjoyable light (= Hypothesis 7 )

This hypothesis is $a$, if not the, predominant hypothesis regarding the enjoyment of negative emotions in recent media studies. In this context, "meaningfulness" is often even associated with the concept of "eudaimonia." Most empirical evidence in favor of this hypothesis comes from this field (Bartsch 2007; 2008; Bartsch \& Viehoff 2003; Bartsch et al. 2010; Oliver 1993; Oliver \& Bartsch 2010; Oliver \& Woolley 2010). Hagtvedt \& Vohs apparently misunderstood our hypothesis as negating a close relation between negative emotions and a search for meaningfulness. However, this nexus is precisely the reason why we included meaningfulness in our model, namely, as one of the means of coping with negative emotions and even integrating them into an overall positive appreciation of artworks. We agree with Hagtvedt \& Vohs that searches for meaning in art contexts are less closely related to straightforward pleasantness than to interest (Silvia 2005a; 2010; see also Knoop et al. 2016) and other types of cognitive and affective responses that allow embracing challenging input, such as being fascinated (Oosterwijk et al. 2016; Rimé et al. 2005).

R2.4.5. Activation of particular genre scripts can contribute to (re)appraising negative emotions in a positive and enjoyable light (= Hypothesis 8 )

In our article, we exemplify this hypothesis with respect to the higher tolerance of dire situations of need and violence primed by the normative happy ending of fairy tales. Other genres, such as tales of horror, entail no such a priori anticipations of a happy turn of events. Still, both genres can be enjoyed, but typically for different reasons and by individuals of different ages, depending on their familiarity with and preference for the respective genres.

The commentaries regarding this hypothesis propose to extend it by including additional variables. In a commentary that goes to the very center of our argument and significantly enriches it, Tan \& Visch propose that the activation of acquired schemata for particular artworks and media products may not only bear on issues of affective valence, but may also contribute to positive evaluation by virtue of being knowledge shared, for instance, by a filmmaker and his or her audience, and hence by establishing a communicative bond. Armstrong \& Cutting suggest that the predictive power that comes with a genre script and the satisfaction derived from its fulfillment may already in itself independently of valence - drive feelings of reward. Obviously, all of these variants of the genre-based hypothesis are not mutually exclusive, but may well apply to different degrees to different genres and different audiences.

\section{R3. Commentaries addressing issues that are included in our model, but not treated in the article}

From the outset, our article acknowledges that several important variables of art reception are not treated in the article, even though they are included in our model's schema. Specifically, we identified "individual differences (personality traits, aesthetic preferences, familiarity, expertise, current mood states)" and "social, cultural, and situational variables of art reception" (see Fig. 1 of target article) as variables that also need to be considered in all specific cases of enjoying negative emotions in art reception. We likewise noted that we deliberately did not treat potential functional benefits - other than the immediate processing pleasure-that might result from enjoyable exposure to art-elicited negative emotions.

Many of the commentaries fill this gap, offering insights and suggestions regarding how these variables might play a role specifically in responses to artworks involving substantial levels of negative emotions. Thus, Hershfield \& Alter offer a rich and empirically well-supported perspective on the importance of situational factors at both the personal and the macroeconomic, social, and political levels, including some interesting hints regarding empirical evidence for functional benefits resulting from frequent exposure to mixed emotions (Adler \& Hershfield 2012; Larsen et al. 2003). Similarly, Leder \& Schwarz convincingly argue that well-established general factors of aesthetic likingin particular, familiarity, expertise, and the resulting differences in fluency - are also likely to have an emotion-regulatory effect on the compatibility of negative emotions with aesthetic enjoyment (see also Brattico \& Vuust). Green \& Fitzgerald provide evidence that the distancing implication of a fiction framing may actually increase prosocial behavior compared with a reality framing. Other commentaries bear on person-related variables for the phenomenon under consideration (Fayn \& Kuppens; Vuoskoski \& Eerola) and on the power that authorial intentions and strategies may exert over recipients even on a subconscious level (Armstrong \& Cutting). Azevedo \& Tsakiris make a case for considering "embodied simulation" as an important factor in the context of our model. Leddington suggests that our model may be helpful for understanding phenomena beyond the confines of the arts proper, specifically, theatrical magic.

Several commentaries stress the importance of social sharing and prosocial relatedness for responses to artworks involving negative emotions (Bastian; Egloff; Tan \& Visch). Prosocial values and feelings of social relatedness constitute an integral part of our conceptualization of "being moved" as an attachment or bonding emotion that loads particularly high on appraisals for prosocial norms and self-ideals (Menninghaus et al. 2015b). However, we have not specifically investigated social sharing in this 
context. Bastian reports evidence that sharing negative emotions can not only lead to an increase in affiliative behavior, but also "amplifies both the pleasantness and the unpleasantness of the respective experience." Egloff even makes the strong claim that social sharing is a "necessary" variable in any aesthetic enjoyment associated with negative emotions. Although this strong claim is not supported by the evidence we are aware of, the issue is clearly of great importance and calls for empirical studies that specifically target a distinct contribution of social sharing to the enjoyment of negative and mixed emotions, both in art reception and beyond.

Kreuzbauer suggests that interaction between the artist and the audience is a further sociopragmatic variable that may influence the enjoyment associated with negative emotions. Swain \& Ho offer reasons why our model may even apply to adaptive parental responses to (otherwise annoying) baby cries, responses that in turn are beneficial for the baby's future life trajectory.

We welcome these additions to our core model, but refrain from any detailed comment, because no direct incompatibility with our model is involved and because we lack expertise regarding some of the fields referred to in the commentaries.

\section{R4. Commentaries that raise issues that are not part of our model}

\section{R4.1. Can the enjoyment of negative emotions be comprehensively explained by the mechanisms of prediction error reduction?}

Following up on an earlier article (Van de Cruys \& Wagemans 2011), Van de Cruys, Chamberlain, \& Wagemans (Van de Cruys et al.) propose that the understanding of the human brain as consistently engaged in predictive coding may parsimoniously explain the enjoyment of negative emotions in art reception (see also Armstrong \& Cutting; Azevedo and Tsakiris; Nadal et al.). They argue that "consolation" and "solace" account for much of the emotional reward experienced in art reception. At the same time, they adopt a widely shared (modern) understanding of the arts that can barely be derived from the hypothesis of predictive coding only: "good artworks will also (temporarily) obstruct alignment" (Van de Cruys et al., para. 6). The resulting challenges (uncertainties) then trigger a process in which viewers strive for a reduction of their prediction error. If successful, such attunement, or regained homeostasis, "yields positive affect (even if the content of the inputs is about negative events)" (para. 6).

For the affective reward of uncertainty reduction, it is thus irrelevant whether the resolved uncertainty is arrived at in a happy ending, as in comedy, or a sad ending, as in tragedy. In this regard, Van de Cruys et al.'s hypothesis is in line with formalist approaches to artworks and actually very close to a formalist analysis of the musical and narrative tension-resolution trajectories that we discuss in section 4.1 of our article. However, their commentary entails no prediction as to why negative affect resulting from prediction errors (uncertainties) should be an inherently enjoyable ingredient of art reception rather than only a somehow necessary obstacle (prediction error) to be overcome on the road to consolation, with the pleasure primarily, if not exclusively, resulting from reducing the prediction error and its concomitant negative effect. Moreover, the prediction error reduction hypothesis cannot readily account for the enjoyment of artworks specifically designed to resist the resolution of ambiguities or other uncertainties (Knobloch-Westerwick \& Keplinger 2006).

The prime affective correlates of cognitive predictions and prediction errors are emotions related to cognitive mastery (Leder \& Nadal 2014), ranging from the pleasures of successful coping to unresolved surprise, frustration, and outright annoyance (if the prediction error reduction fails). The latter are well established as nonpleasurable responses to artworks that are overdemanding to the point of being incompatible with enjoyment. It is less clear, however, how the prediction error (reduction) account of aesthetic enjoyment might explain the role of sadness or of being moved. After all, more often than not, these emotions have a strong bearing on feelings of social relatedness; such feelings cannot readily result from a purely formal successful coping with challenging visual or other compositional patterns. Thus, the theory of predictive codingdriven positive and negative affect is by no means co-extensive with the classical issue of the enjoyment of negative emotion, and Van de Cruys and Wagemans acknowledged this in their 2011 article. In any event, negotiating the overlap and the borders between this elegant, but largely purely formalist theory and our broader model, which also covers form-content interaction, would clearly be an interesting endeavor.

\section{R4.2 Empathy, identification, immersion, transportation}

Vuoskoski \& Eerola stress that empathy, identification, and states of immersion and transportation may be further important variables in emotional responses to artworks. We do not challenge this view. Regarding empathy, we note that it is, in fact, included in our model, if only as an ingredient involved in many instances of being moved. Moreover, a very recent neuroscientific study might be interpreted as suggesting that empathy affects negative emotions in a distinct fashion (Ishizu \& Zeki 2017; see also the commentary by Gerger et al.).

Regarding immersion, transportation, and identification, we have deliberately refrained from including these psychological mechanisms for two reasons. First, we know of no empirical evidence yet that they specifically play a role in rendering negative emotions enjoyable in contexts of art reception. Second, because these mechanisms are related primarily to narrative art forms, they would have even strengthened the bias in favor of literature and film found in larger sections of our article and, hence, potentially compromise its more general claims. Still, as suggested earlier in this Response, we are sympathetic with the idea.

\section{R4.3 The role of historical learning and artistic misunderstanding}

Bullot \& Reber deplore that our model cannot account for how misunderstandings of an artist's intentions and historical learning regarding an artwork's context might affect the mechanisms of Distancing and Embracing. We readily acknowledge that our model does not include special provisions for such variables other than the black box on the 
References/Menninghaus et al.: The Distancing-Embracing model of the enjoyment of negative emotions in art reception

left-hand side, which provides room for an undetermined variety of "social, cultural, and situational variables of art reception."

We have also said very little about artworks "intended to prevent or at least inhibit embracing." At the same time, by no means have we thereby denied such works their status as art. Our model is not at all about defining what is art and what is not. It exclusively hypothesizes: If we enjoy artworks involving negative emotions, then Distancing and Embracing typically are necessary processing factors. Undoubtedly, the well-made and often beautiful shields of ancient and current warriors are not likely to trigger aesthetic enjoyment supported by Distancing and Embracing in their enemies, at least not during fighting. However, in a peaceful environment, the mechanisms stipulated by our model could well become operative, and the same warriors might aesthetically enjoy the sight of these shields even if they depict the killing of enemies. Bullot \& Reber's anecdotal reference to a beholder who reportedly discovered beauty in Damien Hirst's artwork consisting of a cow head infested with maggots likewise speaks in favor of, rather than against our model. We hypothesize that this beholder both distanced and embraced (and, thus, was able to enjoy) the potentially disgusting aspects of the exhibit and, hence, that the predictive power of our model may be stronger than Hirst's (presumable) intentions to elicit aversive feelings in viewers.

\section{R5. Conclusion}

The wealth of commentaries from a variety of disciplines has provided opportunities for further clarification, added several important distinctions, sketched additional variables for potential inclusion in the Embracing factor, and identified interesting venues for future research guided by our model. All in all, we found - using the terminology of our model-far more reason to "embrace" the remarks offered and the propositions made than to "distance" them. Again, we are grateful to the respondents for making the potential privilege of being a Behavioral and Brain Sciences "target" a real one for us. We look forward to a continuation of this process.

\section{References}

[The letters " $a$ " and “ $r$ " before author's initials stand for target article and response references, respectively]

Abelson, R. P. (1981) Psychological status of the script concept. American Psychologist 36(7):715-29. Available at: http://dx.doi.org/10.1037/0003-066X.36.7.715. [NJB, aWM]

Acee, T. W., Kim, H., Kim, H. J., Kim, J., Hsiang-Ning, R. C., Kim, M., Cho, Y. \& Wicker, F. W. (2010) Academic boredom in under- and over-challenging situations. Contemporary Educational Psychology 35(1):17-27. [AE]

Adler, J. M. \& Hershfield, H. E. (2012) Mixed emotional experience is associated with and precedes improvements in psychological well-being. PLOS ONE 7(4): e3563. Available at: http://doi.org/10.1371/journal.pone.0035633. [HEH, rWM]

Aikin, J. \& Aikin, A. L. (1773) On the pleasure derived from objects of terror. In: Miscellaneous pieces, in prose, ed. J. Aikin \& A. L. Aikin, pp. 119-37. Johnson. [aWM]

Ainslie, G. (2005) Précis of breakdown of will. Behavioral and Brain Sciences 28 (5):635-73. [GA]

Ainslie, G. (2013) Money as MacGuffin: A factor in gambling and other process addictions. In: Addiction and self-control: Perspectives from philosophy, psychology, and neuroscience, ed. N. Levy, pp. 16-37. Oxford University Press. [GA]

Ainslie, G. (2017) De gustibus disputare: Hyperbolic delay discounting integrates five approaches to choice. Journal of Economic Methodology 24(2):166-89. [GA]

Alter, A. L. (2013a) Drunk tank pink. Penguin. [HEH]

Alter, A. L. (2013b) The benefits of cognitive disfluency. Current Directions in Psychological Science 22:437-42. [HEH]

Alter, A. L. \& Forgas, J. P. (2007) On feeling happy but fearing failure: The effects of mood on self-handicapping strategies. Journal of Experimental Social Psychology 43:947-54. [HEH]

Alter, A. L. \& Oppenheimer, D. M. (2008) Easy on the mind, easy on the wallet: The roles of familiarity and processing fluency in valuation judgments. Psychonomic Bulletin and Review 15(5):985-90. Available at: http://dx.doi.org/10.3758/PBR. 15.5.985. [aWM]

Altmann, U., Bohrn, I. C., Lubrich, O., Menninghaus, W. \& Jacobs, A. M. (2014) Fact vs fiction - How paratextual information shapes our reading processes. Social Cognitive and Affective Neuroscience 9(1):22-29. Available at: http://dx. doi.org/10.1093/scan/nss098. [aWM]

Aluja-Fabregat, A. (2000) Personality and curiosity about TV and films violence in adolescents. Personality and Individual Differences 29(2):379-92. [KF]

Andrade, E. B. \& Cohen, J. B. (2007) On the consumption of negative feelings. Journal of Consumer Research 34(3):283-300. Available at: http://dx.doi.org/10. 1086/519498. [aWM]

Anz, T. (1998) Spannungskunst und Glückstechniken. In: Literatur und Lust, ed. T. Anz, pp. 150-71. Beck. [aWM]

Apperly, I. A. \& Butterfill, S. A. (2009) Do humans have two systems to track beliefs and belief-like states? Psychological Review 116(4):953. [TRG]

Apter, M. J. (1992) The dangerous edge. The psychology of excitement. Free Press. [aWM]

Apter, M. J. (1993) Phenomenological frames and the paradoxes of experience. In: Advances in reversal theory, ed. J. H. Kerr, S. J. Murgatroyd \& M. J. Apter, pp. 27-39. Swets \& Zeitlinger. [aWM]

Arieti, J. A. \& Crossett, J. M., trans. (1985) Longinus on the sublime. Mellen. [VJK]

Aristotle (1961) Poetics. Trans. S. H. Butcher. Hill \& Wang. [aWM]

Aristotle (1972) Poetics (revised), ed. D. W. Lucas. Clarendon. [SD]

Arnheim, R. (1954) Art and visual perception: A psychology of the creative eye. University of California Press. [AC]

Arnheim, R. (1971) Art and visual perception. University of California Press. [GCC]

Arnheim, R. (1985) The other Gustav Theodor Fechner. In: A century of psychology as science, ed. S. Koch \& D. E. Leary, pp. 856-65. McGraw-Hill. [GCC]

Arnold, M. B. (1960) Emotion and personality. Psychological aspects. Columbia University Press. [aWM]

Aydede, M. (2014) How to unify theories of sensory pleasure: An adverbialist proposal. Review of Philosophy and Psychology 5:119-33. [NFB]

Azevedo, R. T., Garfinkel, S. M., Critchley, H. D. \& Tsakiris, M. (2017) Cardiac afferent activity modulates the expression of racial stereotypes. Nature Communications 8:13854. [RTA]

Baltes, F. R. \& Miu, A. C. (2014) Emotions during live music performance: Links with individual differences in empathy, visual imagery, and mood. Psychomusicology: Music, Mind, and Brain 24:58-65. Available at: http://doi.org/10.1037/ pmu0000030. [GG]

Barbalet, J. M. (1999) Boredom and social meaning. The British Journal of Sociology 50(4):631-46. [AE]

Barford, K., Fayn, K., Silvia, P. J. \& Smilie, L. (in press) Individual differences in conflicting stimulus evaluations: Openness/intellect predicts mixed-valenced appraisals of visual art. Journal of Research and Personality. [KF]

Barford, K. A. \& Smillie, L. D. (2016) Openness and other Big Five traits in relation to dispositional mixed emotions. Personality and Individual Differences 102:118-22. [KF]

Barrett, L. F. (2017) How emotions are made: The secret life of the brain. Houghton Mifflin Harcourt. [GCC]

Barrett, L. F. \& Bar, M. (2009) See it with feeling: Affective predictions during object perception. Philosophical Transactions of the Royal Society of London B: Biological Sciences 364:1325-34. [MN]

Barrett, L. F., Gross, J., Christensen, T. C. \& Benvenuto, M. (2001) Knowing what you're feeling and knowing what to do about it: Mapping the relation between emotion differentiation and emotion regulation. Cognition and Emotion 15:713-24. [NFB]

Barrett, L. F., Mesquita, B., Ochsner, K. N. \& Gross, J. J. (2007) The experience of emotion. Annual Review of Psychology 58:373-403.

Barrett, L. F., Quigley, K. S., Hamilton, P. (2016) An active inference theory of allostasis and interoception in depression. Philosophical Transactions of the Royal Society of London B: Biological Sciences 371(1708):20160011. [RTA]

Barrett, L. F. \& Wager, T. (2006) The structure of emotion: Evidence from the neuroimaging of emotion. Current Directions in Psychological Science 15:7985. $[\mathrm{MN}]$ 
References/Menninghaus et al.: The Distancing-Embracing model of the enjoyment of negative emotions in art reception

Barrett, N. F. \& Schulkin, J. (under review). A neurodynamic perspective on musical enjoyment: The role of emotional granularity. [NFB]

Barsalou, L. W. (2003) Abstraction in perceptual symbol systems. Philosophical Transactions of the Royal Society of London B: Biological Sciences 358 (1435):1177-87. [EST]

Barsalou, L. W. (2008) Grounded cognition. Annual Review of Psychology 59:61745. [EST]

Bartsch, A. (2007) Meta-emotion and genre-preference. What makes horror films and tear-jerkers enjoyable? In: Narration and spectatorship in moving images, ed. J. D. Anderson \& N. Fisher Anderson, pp. 124-35. Cambridge Scholars. [arWM]

Bartsch, A. (2008) Meta-emotion: How films and music videos communicate emotions about emotions. Projections 2(1):45-59. [arWM]

Bartsch, A., Appel, M. \& Storch, D. (2010) Predicting emotions and meta-emotions at the movies: The role of the need for affect in audiences' experience of horror and drama. Communication Research 37(2):167-90. Available at: http://doi.org/ 10.1177/0093650209356441. [arWM]

Bartsch, A. \& Viehoff, R. (2003) Meta-emotion: In search of a meta-account for entertainment by negative emotions. SPIEL 22(2):309-28. [arWM]

Bartsch, A., Vorderer, P., Mangold, R. \& Viehoff, R. (2008) Appraisal of emotions in media use: Toward a process model of meta-emotion and emotion regulation. Media Psychology 11(1):7-27. Available at: http://dx.doi.org/10.1080/ 15213260701813447. [aWM]

Bastian, B., Jetten, J. \& Ferris, L. J. (2014a) Pain as social glue: Shared pain increases cooperation. Psychological Science 25:2079-85. [BB]

Bastian, B., Jetten, J., Hornsey, M. J. \& Leknes, S. (2014b) The positive consequences of pain: A biopsychosocial approach. Personality and Social Psychology Review 18:256-79. [BB]

Batteux, C. (1746/2015) The fine arts reduced to a single principle, trans. J. O. Young. Oxford University Press. [aWM]

Baumeister, R. F., Bratslavsky, E., Finkenauer, C. \& Vohs, K. D. (2001) Bad is stronger than good. Review of General Psychology 5(4):323-70. Available at: http://dx.doi.org/10.1037/1089-2680.5.4.323. [aWM]

Baumeister, R. F., Vohs, K. D., Aaker, J. L. \& Garbinsky, E. N. (2013) Some key differences between a happy life and a meaningful life. The Journal of Positive Psychology 8:505-16. [HH]

Bench, S. W. \& Lench, H. C. (2013) On the function of boredom. Behavioral Sciences 3(3):459-72. [AE]

Benedek, M. \& Kaembach, C. (2011) Physiological correlates and emotional specificity of human piloerection. Biological Psychology 86(3):320-29. Available at: http://dx.doi.org/10.1016/j.biopsycho.2010.12.012. [aWM]

Benedetti, J. (2007) The art of the actor: The essential history of acting, from classical times to the present day. Theatre Arts Book. [TRG]

Berenbaum, H. (2002) Varieties of joy-related pleasurable activities and feelings Cognition \& Emotion 16(4):473-94. Available at: http://dx.doi.org/10.1080/ 0269993014000383. [aWM]

Berlyne, D. E. (1960) Conflict, arousal, and curiosity. McGraw-Hill. [aWM]

Berlyne, D. E. (1970) Aesthetics and psychobiology. Appleton Century Crofts. [GG]

Berlyne, D. E. (1971a) Aesthetics and psychobiology. Meredith. [aWM]

Berlyne, D. E. (1971b) Aesthetics and psychobiology. Appleton-Century Croft. [AC]

Berlyne, D. E., ed. (1974) Studies in the new experimental aesthetics. Steps toward an objective psychology of aesthetic appreciation. Wiley. [aWM]

Bezdek, M. A., Gerrig, R. J., Wenzel, W. G., Shin, J., Pirog Revill, K. \& Schumacher, E. H. (2015) Neural evidence that suspense narrows attentional focus. Neuroscience 303:338-45. Available at: http://dx.doi.org/10.1016/j.neuroscience.2015. 06.055. [KLA]

Bless, H., Fiedler, K. \& Forgas, J. P. (2006) Mood and regulation of information processing and behavior. In: Affect in social thinking and behavior, ed. J. P. Forgas, pp. 65-84. Psychology Press. [HEH]

Blood, A. J. \& Zatorre, R. J. (2001) Intensely pleasurable responses to music correlate with activity in brain regions implicated in reward and emotion. Proceedings of the National Academy of Sciences of the United States of America 98 (20):11818-23. Available at: http://dx.doi.org/10.1073/pnas.191355898. [RTA, aWM]

Bloom, P. (2011) How pleasure works: The new science of why we like what we like. Norton. $[\mathrm{aWM}]$

Bloom, P. (2016) Against empathy: The case for rational compassion. Ecco Press. $[\mathrm{PCH}]$

Blount, J. D., Metcalfe, N. B., Birkhead, T. R. \& Surai, P. F. (2003) Carotenoid modulation of immune function and sexual attractiveness in zebra finches. Science 300(5616): 125-27. [NR]

Bollen, J., Pepe, A. \& Mao, H. (2009) Modeling public mood and emotion: Twitter sentiment and socio-economic phenomena. In: Proceedings of the Fifth International AAAI Conference on Weblogs and Social Media (ICWSM 2011), 17-21 July 2011, Barcelona, Spain. Association for the Advancement of Artificial Intelligence. arXiv:0911.1583. [HEH]

Boothby, E. J., Clark, M. S. \& Bargh, J. A. (2014) Shared experiences are amplified. Psychological Science 25(12):2209-16. [BB]
Bordwell, D. (1985) Narration in the fiction film. University of Wisconsin Press. [EST]

Bordwell, D. (2002) Intensified continuity: Visual style in contemporary American film. Film Quarterly 55(3):16-28. Available at: http://dx.doi.org/10.1525/fq. 2002.55.3.16. [KLA]

Bordwell, D. (2006) The way Hollywood tells it. University of California Press. [KLA]

Bornstein, R. F. (1989) Exposure and affect: Overview and meta-analysis of research, 1968-1987. Psychological Bulletin 106(2):265. Available at: http://dx.doi.org/10. 1037/0033-2909.106.2.265. [aWM]

Bowlby, J. (1969) Attachment and loss: Vol. 1. Attachment. Hogarth. [JES]

Bowlby, J. (1973) Attachment and loss: Vol. 2. Separation: Anxiety and anger. Basic. [JES]

Brattico, E. (2015) From pleasure to liking and back: Bottom-up and top-down neural routes to the aesthetic enjoyment of music. In: Art, aesthetics, and the brain, ed. M. Nadal, J. P. Houston, L. Agnati, F. Mora \& C. J. Cela Conde, pp. 303-18. Oxford University Press. [EB]

Brattico, E., Bogert, B., Alluri, V., Tervaniemi, M., Eerola, T. \& Jacobsen, T. (2016) It's sad but I like it: The neural dissociation between musical emotions and liking in experts and laypersons. Frontiers in Human Neuroscience 9:676. Available at: http://doi.org/10.3389/fnhum.2015.00676. [EB, rWM]

Brattico, E., Bogert, B. \& Jacobsen, T. (2013) Toward a neural chronometry for the aesthetic experience of music. Frontiers in Psychology 4:206. Available at: http://doi.org/10.3389/fpsyg.2013.00206. [EB, aWM]

Brattico, E., Brattico, P. \& Jacobsen, T. (2009-2010) The origins of the aesthetic enjoyment of music - A review of the literature. Musicae Scientiae, Special Issue 2009-2010:15-31. [EB]

Brattico, E. \& Jacobsen, T. (2009) Subjective appraisal of music: Neuroimaging evidence. Annals of the New York Academy of Sciences 1169:308-17. Available at: http://doi.org/10.1111/j.1749-6632.2009.04843.x. [EB]

Brattico, E., Jacobsen, T., De Baene, W., Glerean, E. \& Tervaniemi, M. (2010) Cognitive vs. affective listening modes and judgments of music - An ERP study. Biological Psychology 85(3):393-409. Available at: http://doi.org/10.1016/j. biopsycho.2010.08.014. [EB]

Brewer, W. F. \& Lichtenstein, E. H. (1982) Stories are to entertain: A structuralaffect theory of stories. Journal of Pragmatics 6(5-6):473-86. Available at: http://dx.doi.org/10.1016/0378-2166(82)90021-2. [aWM]

Brewer, W. F. \& Nakamura, G. V. (1984) The nature and functions of schemas. Center for the Study of Reading Technical Report No. 325. University of Illinois at Urbana-Champaign. [aWM]

Brown, J. L., Sheffield, D., Leary, M. R. \& Robinson, M. E. (2003) Social support and experimental pain. Psychosomatic Medicine 65(2):276-83. [BB]

Brown, S. L. \& Brown, R. M. (2015) Connecting prosocial behavior to improved physical health: Contributions from the neurobiology of parenting. Neuroscience and Biobehavioral Reviews 55:1-17. Available at: http://doi.org/10.1016/j. neubiorev.2015.04.004. [JES]

Brunner, J. (2017) Why immersive theatre isn't just a fad. Playbill, April 22. Available at: http://www.playbill.com/article/why-immersive-theatre-isnt-just-a-fad. [TRG]

Bullot, N. J. \& Reber, R. (2013a) The artful mind meets art history: Toward a psychohistorical framework for the science of art appreciation. Behavioral and Brain Sciences 36(2):123-37. Available at: http://doi.org/10.1017/ S0140525X12000489. [NJB, AC]

Bullot, N. J. \& Reber, R. (2013b) A psycho-historical research program for the integrative science of art. Behavioral and Brain Sciences 36(2):163-80. Available at: http://doi.org/10.1017/S0140525X12002464. [NJB, aWM, NR]

Bullot, N. J., Seeley, W. P. \& Davies, S. (in press) Art and science: A philosophical sketch of their historical complexity and co-dependence. Journal of Aesthetics and Art Criticism. [NJB]

Bullough, E. (1912) 'Psychical distance' as a factor in art and an aesthetic principle. British Journal of Psychology 5(2):87-118. Available at: http://dx.doi.org/10. 1111/j.2044-8295.1912.tb00057.x. [GCC, SD, aWM]

Bundgaard, H. (2015) Feeling, meaning, and intentionality - A critique of the neuroaesthetics of beauty. Phenomenology and the Cognitive Sciences 14:781-801. [EB]

Burke, E. (1757) A philosophical enquiry into the origin of our ideas of the sublime and beautiful. The Project Gutenberg EBook of The Works of the Right Honourable Edmund Burke. [GG]

Burke, E. (1759/1971) A philosophical enquiry into the origin of our ideas of the sublime and beautiful, 2nd edition. Garland. [VJK]

Burwick, F. (1991) Illusion and the drama: Critical theory of the enlightenment and romantic era. Pennsylvania State University. [GCC]

Busselle, R. \& Bilandzic, H. (2008) Fictionality and perceived realism in experiencing stories: A model of narrative comprehension and engagement. Communication Theory 18(2):255-80. DOI: 10.1111/j.1468-2885.2008.00322.x . [aWM]

Cacioppo, J. T. \& Gardner, W. L. (1999) Emotion. Annual Review of Psychology 50:191-214. Available at: http://dx.doi.org/10.1146/annurev.psych.50.1.191. [aWM] 
Cacioppo, J. T., Gardner, W. L. \& Berntson, G. G. (1999) The affect system has parallel and integrative processing components: Form follows function. Journal of Personality and Social Psychology 76(5):839-55. Available at: http://dx.doi. org/10.1037/0022-3514.76.5.839. [aWM]

Calvo-Merino, B., Glaser, D. E., Grezes, J., Passingham, R. E. \& Haggard, P. (2005) Action observation and acquired motor skills: An fMRI study with expert dancers. Cerebral Cortex 15(8):1243-49. [RTA]

Cameron, C. D. \& Payne, B. K. (2011) Escaping affect: How motivated emotion regulation creates insensitivity to mass suffering. Journal of Personality and Social Psychology 100:1-15. Available at: http://doi.org/10.1037/a0021643. [MCG]

Carrera, P. \& Oceja, L. (2007) Drawing mixed emotions: Sequential or simultaneous experiences? Cognition and Emotion 21(2):422-41. Available at: http://doi.org/ 10.1080/02699930600557904. [arWM]

Carroll, N. (1990a) The philosophy of horror or paradoxes of the heart. Routledge. [SD]

Carroll, N. (1990b) The philosophy of horror or paradoxes of the heart. Taylor \& Francis. [aWM]

Carroll, N. (1996) Toward a theory of film suspense. In: Theorizing the moving image, ed. N. Carroll, pp. 94-115. Cambridge University Press. [aWM]

Carroll, N. (2001) Beyond aesthetics: Philosophical essays. Cambridge University Press. [NJB]

Cartier-Bresson, H. C. (1952) The decisive moment. Simon and Schuster. [AC]

Chartrand, T. L. \& Bargh, J. A. (1999) The chameleon effect: The perceptionbehavior link and social interaction. Journal of Personality and Social Psychology 76(6):893-910. [TRG]

Chatterjee, A. (2004a) The neuropsychology of visual artists. Neuropsychologia 42:1568-83. Available at: http://dx.doi.org/10.1016/j.neuropsychologia.2004.03. 011. [AC]

Chatterjee, A. (2004b) Prospects for a cognitive neuroscience of visual aesthetics. Bulletin of Psychology and the Arts 4:55-59. [AC]

Chatterjee, A. (2013) The aesthetic brain: How we evolved to desire beauty and enjoy art. Oxford University Press. [aWM]

Chatterjee, A. (2014) Neuroaesthetics: Growing pains of a new discipline. In: Aesthetic science: Connecting minds, brains, and experience, ed. A. Shimamura \& S. Palmer, pp. 299-317. Oxford University Press. [PCH]

Chatterjee, A. \& Vartanian, O. (2014) Neuroaesthetics. Trends in Cognitive Sciences 18(7):370-75. [NR]

Chatterjee, A. \& Vartanian, O. (2016) Neuroscience of aesthetics. Annals of the New York Academy of Sciences 1369(1):172-94. Available at: http://doi.org/10. 1111/nyas.13035. [AC]

Chekhov, A. (1899) The lady with the toy dog. In: Anton Chekhov: Five great short stories, trans. S. S. Koteliansky \& G. Cannan. pp. 81-94. Dover (current edition 1990). [KO]

Christensen, J. F., Gomila, A., Gaigg, S. B., Sivarajah, N. \& Calvo-Merino, B. (2016) Dance expertise modulates behavioural and psychophysiological responses to affective body movement. Journal of Experimental Psychology 42(8):1139-47. [RTA]

Cikara, M., Bruneau, E. G. \& Saxe, R. (2011) Us and them: Intergroup failures of empathy. Current Directions in Psychological Sciences 20(3):149-53. [RTA]

Clark, A. (2013) Whatever next? Predictive brains, situated agents, and the future of cognitive science. Behavioral and Brain Sciences 36(3):181-204. Available at: https://doi.org/10.1017/S0140525X12000477. [RTA, SVdC]

Clarke, E., DeNora, T. \& Vuoskoski, J. (2015) Music, empathy and cultural understanding. Physics of Life Reviews 15:61-88. [JKV]

Cohen, M. (1965) Aesthetic essence. In: Philosophy in America, ed. M. Black, pp 115-33. Allen \& Unwin. [SD]

Collingwood, R. G. (1938) The principles of art. Oxford University Press. [KO]

Coplan, A. (2006) Catching characters emotions: Emotional contagion responses to narrative fiction film. Film Studies 8(8):26-38. [TRG]

Cosmides, L. \& Tooby, J. (2000) Adaptations for decoupling and metarepresentation. In: Metarepresentations: A multidisciplinary perspective, ed. D. Sperber. Oxford. [NR]

Cotter, K. N., Silvia, P. J. \& Fayn, K. (2017) What does feeling like crying when listening to music feel like? Psychology of Aesthetics, Creativity and the Arts. DOI: 10.1037/aca0000108. [KF]

Critchley, H. D. \& Harrison, N. A. (2013) Visceral influences on brain and behavior Neuron 77(4):624-38. [RTA]

Cross, E. S., Kirsch, L., Ticini, L. F. \& Schütz-Bosbach, S. (2011) The impact of aesthetic evaluation and physical ability on dance perception. Frontiers in Human Neuroscience 5:102. [RTA]

Csikszentmihalyi, M. (1975) Beyond boredom and anxiety. Jossey-Bass. [AE]

Cupchik, G. C. (2002) The evolution of psychical distance as an aesthetic concept. Culture \& Psychology 8(2):155-87. Available at: http://dx.doi.org/10.1177/ 1354067X02008002437. [GCC, aWM]

Cupchik, G. C. (2016) The aesthetics of emotion: Up the down staircase of the mindbody. Cambridge University Press. [GCC]

Cupchik, G. C. \& Wroblewski-Raya, V. (1998) Loneliness as a theme in painting. Visual Arts Research 24:65-71. [GG]
Cutting, J. E. (2015) The framing of characters in popular movies. Art and Perception 3(2):191-212. Available at: http://doi.org/10.1163/22134913-00002031. [KLA]

Cutting, J. E. (2016) Narrative theory and the dynamics of popular movies. Psychonomic Bulletin and Review 23(6):1713-43. Available at: http://doi.org/10. 3758/s13423-016-1051-4. [KLA]

Cutting, J. E., Brunick, K. L. \& Candan, A. (2012) Perceiving event dynamics and parsing Hollywood films. Journal of Experimental Psychology: Human Perception and Performance 38(6):1476-90. Available at: http://doi.org/10.1037/ a0027737. [KLA]

Cutting, J. E., Brunick, K. L., DeLong, J. E., Iricinschi, C. \& Candan, A. (2011) Quicker, faster, darker: Changes in Hollywood film over 75 years. $i$-Perception 2 (6):569-76. Available at: http://doi.org/10.1068/i044laap. [KLA]

Cutting, J. E. \& Candan, A. (2013) Movies, evolution, and mind: From fragmentation to continuity. The Evolutionary Review 4(3):25-35. [KLA]

Cutting, J. E., DeLong, J. E. \& Nothelfer, C. E. (2010) Attention and the evolution of Hollywood film. Psychological Science 21:440-47. Available at: http://doi.org/ 10.1177/0956797610361679. [KLA]

Danckert, J. \& Merrifield, C. (2016) Boredom, sustained attention and the default mode network. Experimental Brain Research. DOI: 10.1007/s00221-016-46175. [AE]

Danto, A. C. (2003) The abuse of beauty: Aesthetics and the concept of art. Open Court. [NJB]

Danziger, K. (1997) Naming the mind: How psychology found its language. Sage. [GCC]

Davies, S. (2006) The philosophy of art. Blackwell. [NJB, NR]

Davies, S. (2009) Responding emotionally to fictions. Journal of Aesthetics and Art Criticism 67:269-84. [SD]

de Fontenelle, B. l. B. (1692/1968) Réflexions sur la poétique. In: CEuvres complètes, vol. 3, ed. G. B. Depping, pp. 1-30. Slatkine Reprints. [aWM]

de Vignemont, F. \& Singer, T. (2006) The empathic brain: How, when and why? Trends in Cognitive Sciences 10(10):435-41. Available at: http://dx.doi.org/10. 1016/j.tics.2006.08.008. [aWM]

de Wied, M., Zillmann, D. \& Ordman, V. (1994) The role of empathic distress in the enjoyment of cinematic tragedy. Poetics 23(1-2):91-106. Available at: http://dx. doi.org/10.1016/0304-422X(94)00010-4. [aWM]

Deonna, J. A. (2011) Etre ému [Being moved]. In: Les ombres de l'âme: Penser les emotions negatives, ed. C. Tappolet, F. Teroni \& A. Konzelman Ziv, pp. 111-28. Haller. [TWS]

Derryberry, D. (1988) Emotional influences on evaluative judgments: Roles of arousal, attention, and spreading activation. Motivation and Emotion 12:23-55. Available at: http://dx.doi.org/10.1007/BF00992471. [GG, SVdC]

DeYoung, C. G. (2014) Openness/Intellect: A dimension of personality reflecting cognitive exploration. In: The APA handbook of personality and social psychology: Personality processes and individual differences, vol. 3, ed. R. J. Larsen $\&$ M. L. Cooper. American Psychological Association. [KF]

Dickie, G. (1964) The myth of the aesthetic attitude. American Philosophical Quarterly 1:56-65. [SD]

Dinesh, N. (2017) Memos from a Theatre Lab: Exploring what immersive theatre 'does'. Routledge. [TRG]

Dissanayake, E. (2000) Antecedents of the temporal arts in early mother-infant interaction. In: The origins of music, ed. N. L. Wallin, B. Merker \& S. Brown, pp. 389-410. MIT Press. [aWM]

Dixon, P. \& Bortolussi, M. (2009) Readers' knowledge of popular genre. Discourse Processes 46(6):541-71. Available at: http://dx.doi.org/10.1080/ 01638530902959570. [aWM]

Djikic, M. \& Oatley, K. (2014) The art in fiction: From indirect communication to changes of the self. Psychology of Aesthetics, Creativity, and the Arts 8:498505. [KO]

Djikic, M., Oatley, K. \& Carland, M. (2012) Genre or artistic merit: The effect of literature on personality. Scientific Study of Literature 2:25-36. [KO]

Djikic, M., Oatley, K. \& Peterson, J. B. (2006) The bitter-sweet labor of emoting: The linguistic comparison of writers and physicists. Creativity Research Journal 18(2):191-97. Available at: http://doi.org/10.1207/s15326934crj1802_5. [rWM, $\mathrm{KO}]$

Djikic, M., Oatley, K., Zoeterman, S. \& Peterson, J. B. (2009) On being moved by art: How reading fiction transforms the self. Creativity Research Journal 21(1):2429. Available at: http://doi.org/10.1080/10400410802633392. [rWM, KO]

Do, A. M., Rupert, A. V. \& Wolford, G. (2008) Evaluations of pleasurable experiences: The peak-end rule. Psychonomic Bulletin \& Review 15(1):96-98. Available at: http://dx.doi.org/10.3758/pbr.15.1.96. [aWM]

Dovidio, J. F., Piliavin, J. A., Gaertner, S. L., Schroeder, D. A. \& Clark, R. D., III. (1991) The arousal: Cost-reward model and the process of intervention: A review of the evidence. In: Review of personality and social psychology: Vol. 12. Prosocial behavior, ed. M. S. Clark, pp. 86-118. Sage. [MCG]

Dubé, L. \& Le Bel, J. L. (2003) The content and structure of laypeople's concept of pleasure. Cognition \& Emotion 17(2):263-95. Available at: http://dx.doi.org/10. 1080/02699930302295. [aWM] 
Durkheim, E. (1995) Les formes élémentaires de la vie religieuse. [The elementary forms of religious life]. Free Press. (Original work published 1912). [BB]

Dutton, D. (2009) The art instinct. Bloomsbury. [NR]

Eastwood, J. D., Frischen, A., Fenske, M. J. \& Smilek, D. (2012) The unengaged mind defining boredom in terms of attention. Perspectives on Psychological Science 7(5):482-95. [AE]

Eaton, M. M. (1982) A strange kind of sadness. Journal of Aesthetics and Art Criticism 41(1):51-63. Available at: http://dx.doi.org/10.2307/430823. [aWM]

Eerola, T. \& Vuoskoski, J. K. (2011) A comparison of the discrete and dimensional models of emotion in music. Psychology of Music 39(1):18-49. Available at: http://dx.doi.org/10.1177/0305735610362821. [aWM]

Eerola, T., Vuoskoski, J. \& Kautiainen, H. (2016) Being moved by unfamiliar sad music is associated with high empathy. Frontiers in Psychology 7:1176. Available at: http://dx.doi.org/10.3389/fpsyg.2016.01176. [aWM, JKV, GG]

Elpidorou, A. (2014) The bright side of boredom. Frontiers in Psychology 5:1245. [AE]

Elpidorou, A. (2015) The quiet alarm. Aeon Magazine. Available at: https://aeon.co/ essays/life-without-boredom-would-be-a-nightmare. [AE]

Elpidorou, A. (2017a) The moral dimensions of boredom: A call for research. Review of General Psychology 21(1):30-48. [AE]

Elpidorou, A. (2017b) The bored mind is a guiding mind: Toward a regulatory theory of boredom. Phenomenology and the Cognitive Sciences. DOI: 10.1007/s11097017-9515-1. [AE]

Elpidorou, A. (2017c) The good of boredom. Philosophical Psychology. DOI: 10.1080/09515089.2017.1346240. [AE]

Elster, J. (1989) Nuts and bolts for the social sciences. Cambridge University Press. [GA]

Ersner-Hershfield, H., Mikels, J. A., Sullivan, S. J. \& Carstensen, L. L. (2008) Poignancy: Mixed emotional experience in the face of meaningful endings. Journal of Personality and Social Psychology 94(1):158-67. Available at: http://dx.doi. org/10.1037/0022-3514.94.1.158. [aWM]

Eskine, K. J., Kacinik, N. A. \& Prinz, J. J. (2012) Stirring images: Fear, not happiness or arousal, makes art more sublime. Emotion 12(5):1071-74. Available at: http:// dx.doi.org/10.1037/a0027200. [aWM]

Fahlman, S. A., Mercer-Lynn, K. B., Flora, D. B. \& Eastwood, J. D. (2013) Development and validation of the multidimensional state boredom scale. Assessment 20:68-85. [AE]

Farmer, R. \& Sundberg, N. D. (1986) Boredom proneness - The development and correlates of a new scale. Journal of Personality Assessment 50(1):4-17. $[\mathrm{AE}]$

Fayn, K., MacCann, C., Tiliopoulos, N. \& Silvia, P. J. (2015) Aesthetic emotions and aesthetic people: Openness predicts sensitivity to novelty in the experiences of interest and pleasure. Frontiers in Psychology 6:1877. [KF]

Fayn, K., Silvia, P. J., Erbas, Y., Tiliopoulos, N. \& Kuppens, P. (in press-a) Nuanced aesthetic emotions: Emotion differentiation is related to knowledge of the arts and curiosity. Cognition and Emotion. http://dx.doi.org/10.1080/ 02699931.2017.1322554. [KF]

Fayn, K., Silvia, P. J., MacCann, C. \& Tiliopoulos, N. (in press-b) Interested in different things or in different ways? Exploring the engagement distinction between openness and intellect. Journal of Individual Differences. [KF]

Feagin, S. L. (1983) The pleasures of tragedy. American Philosophical Quarterly 20 (1):95-104. [SD, aWM]

Fechner, G. T. (1876) Vorschule der Ästhetik. Breitkopf \& Härtel. [AC, aWM]

Feldman Barrett, L. (2004) Feelings or words? Understanding the content in selfreport ratings of experienced emotion. Journal of Personality and Social Psychology 87(2):266-81. Available at: http://doi.org/10.1037/0022-3514.87.2.266. [rWM]

Fenner, D. (1996) The aesthetic attitude. Humanities. [EB]

Fill, A. (2007) Das Prinzip Spannung: Sprachwissenschaftliche Betrachtungen zu einem universalen Phänomen. Narr Francke Attempto. [aWM]

Fischer, A. H. \& Manstead, A. S. R. (2008) Social functions of emotion. In: Handbook of emotions, 3rd edition, ed. M. Lewis, J. M. Haviland-Jones \& L. F. Barrett, pp. 456-70. Guilford. [BE]

Fisher, C. D. (1993) Boredom at work: A neglected concept. Human Relations 46:395-417. [AE]

Fiske, A. P. (1992) The four elementary forms of sociality: Framework for a unified theory of social relations. Psychological Review 99(4):689-723. [TWS]

Fitch, W. T., von Graevenitz, A. \& Nicolas, E. (2009) Bio-aesthetics and the aesthetic trajectory: A dynamic cognitive and cultural perspective. In: Neuroaesthetics, ed. M. Skov \& O. Vartanian, pp. 59-102. Baywood. [aWM]

Fitzgerald, K. \& Green, M. C. (2017) Restorative narrative: A new approach to prosocial media. Unpublished manuscript, University at Buffalo. [MCG]

Fokkinga, S. F. \& Desmet, P. M. A. (2012) Darker shades of joy: The role of negative emotion in rich product experiences. Design Issues 28(4):42-56. Available at: http://doi.org/10.1162/DESI_a_00174. [rWM]

Fokkinga, S. F. \& Desmet, P. M. A. (2013) Ten ways to design for disgust, sadness, and other enjoyments: A design approach to enrich product experiences with negative emotions. International Journal of Design 7(1):19-36. [rWM]
Fong, C. T. (2006) The effects of emotional ambivalence on creativity. Academy of Management Journal 49(5):1016-1030. [HEH]

Forgas, J. (2001) Feeling and thinking: The role of affect in social cognition. Cambridge University Press. [HEH]

Forster, M., Leder, H. \& Ansorge, U. (2016) Exploring the subjective feeling of fluency. Experimental Psychology 63:45-58. Available at: http://doi.org/10. 1027/1618-3169/a000311. [HL]

Fredrickson, B. (2004) The broaden-and-build theory of positive emotions. Philosophical Transactions of the Royal Society of London, Series B: Biological Sciences 359(1449):1367-78. [HEH]

Freedberg, D. \& Gallese, V. (2007) Motion, emotion and empathy in esthetic experience. Trends in Cognitive Sciences 11(5):197-203. Available at: https:// doi.org/10.1016/j.tics.2007.02.003. [RTA, SVdC]

Freud, S. (1955) The uncanny. In: The standard edition of the complete psychological works of Sigmund Freud, vol. 17, trans., A. Strachey \& J. Strachey, ed. J. Strachey, pp. 217-52. Hogarth. [aWM]

Friend, S. (2007) The pleasures of documentary tragedy. British Journal of Aesthetics 47(2):184-98. Available at: http://dx.doi.org/10.1093/aesthj/ayl055. [aWM]

Frijda, N. H. (1988) The laws of emotion. American Psychologist 43(5):349-58. Available at: http://dx.doi.org/10.1037/0003-066x.43.5.349. [arWM, TWS]

Frijda, N. H. \& Sundararajan, L. (2007) Emotion refinement: A theory inspired by Chinese poetics. Perspectives on Psychological Science 2(3):227-41. Available at: http://doi.org/10.1111/j.1745-6916.2007.00042.x. [rWM]

Friston, K. J. (2010) The free-energy principle: A unified brain theory? Nature Reviews Neuroscience 11(2):127-38. Available at: https://doi.org/10.1038/ nrn2787. [SVdC]

Friston, K. J. \& Frith, C. D. (2015) Active inference, communication and hermeneutics. Cortex 68:129-43. Available at: https://doi.org/10.1016/j.cortex.2015. 03.025. [SVdC]

Fukui, H. \& Toyoshima, K. (2014) Chill-inducing music enhances altruism in humans. Frontiers in Psychology: Cognition 5:1215. Available at: http://dx.doi org/10.3389/fpsyg.2014.01215. [aWM]

Furnham, A. \& Bunyan, M. (1988) Personality and art preferences. European Journal of Personality 2(1):67-74. [KF]

Gabrielsson, A. (2011) Strong experiences with music, trans. R. Bradbury. Oxford University Press. [NFB]

Gabrielsson, A. \& Wik, S. L. (2003) Strong experiences related to music: A descriptive system. Musicae Scientiae 7(2):157-217. [NR]

Gallese, V. \& Sinigaglia, C. (2011) What is so special about embodied simulation? Trends in Cognitive Sciences 15(11):512-19. [RTA]

Game, A. M. (2007) Workplace boredom coping: Health, safety, and HR implications. Personnel Review 36:701-21. [AE]

Garg, N., Wansink, B. \& Inman, J. J. (2007) The influence of incidental affect on consumers' food intake. Journal of Marketing 71(1):194-206. [HEH]

Garner, W. R. (1962) Uncertainty and structure of psychological concepts. Wiley. [GCC]

Garrido, S. \& Schubert, E. (2011) Individual differences in the enjoyment of negative emotion in music: A literature review and experiment. Music Perception: An Interdisciplinary Journal 28(3):279-96. Available at: http://dx.doi.org/10.1525/ mp.2011.28.3.279. [GG, aWM]

Gaut, B. (1993) The paradox of horror. British Journal of Aesthetics 33:333-45. [SD]

Gaut, B. (2003) Reasons, emotions, and fictions. In: Imagination, philosophy, and the arts, ed. M. Kieran \& D. M. Lopes, pp. 15-34. Routledge. [aWM]

Gebauer, L. K., Kringelbach, M. L. \& Vuust, P. (2012) Ever-changing cycles of musical pleasure: The role of dopamine and anticipation. Psychomusicology 22 (2):152-67. [EB]

Geng, X., Chen, Z., Lam, W. \& Zheng, Q. (2013) Hedonic evaluation over short and long retention intervals: The mechanism of the peak-end rule. Journal of Behavioral Decision Making 26(3):225-36. Available at: http://dx.doi.org/10. 1002/bdm.1755. [aWM]

Gerbner, G. \& Gross, L. (1976) The scary world of TV's heavy viewer. Psychology Today 9(11):41-45. [TRG]

Gerger, G., Leder, H. \& Kremer, A. (2014) Context effects on emotional and aesthetic evaluations of artworks and IAPS pictures. Acta Psychologica 151(1):174 83. Available at: http://doi.org/10.1016/j.actpsy.2014.06.008. [VJK, arWM, MN NR]

Gerger, G., Pelowski, M., \& Leder, H. (2017). Erratum to: Empathy, Einfühlung, and aesthetic experience: The effect of emotion contagion on appreciation of representational and abstract art using fEMG and SCR. Cognitive Processing. Available at: https://doi.org/10.1007/s10339-017-0807-8. [GG]

Gernot, G., Pelowski, M. \& Leder, H. (2017) Empathy, Einfühlung, and aesthetic experience: The effect of emotion contagion on appreciation of representational and abstract art using fEMG and SCR. Cognitive Processing. Available at: http:/ doi.org/10.1007/s10339-017-0800-2. [GG, HL]

Gerrig, R. J. (1997) Is there a paradox of suspense? A reply to Yanal. British Journal of Aesthetics 37(2):168-75. Available at: http://dx.doi.org/10.1093/bjaesthetics/ 37.2.168. [aWM]

Gilbert, D. T. (1991) How mental systems believe. American Psychologist 46(2):107. $[\mathrm{NR}]$ 
Giuliani, N. R. \& Gross, J. J. (2009) Reappraisal. In: Oxford companion to the affective sciences, ed. D. Sander \& K. R. Scherer, pp. 329-30. Oxford University Press. [aWM]

Glucklich, A. (2001) Sacred pain: Hurting the body for the sake of the soul. Oxford University Press. [NFB]

Goldenberg, J. L., Pyszczynski, T., Johnson, K. D., Greenberg, J. \& Solomon, S. (1999) The appeal of tragedy: A terror management perspective. Media Psychology 1(4):313-29. Available at: http://dx.doi.org/10.1207/ s1532785xmep0104_2. [aWM]

Goldstein, A. (1980) Thrills in response to music and other stimuli. Physiological Psychology 8(1):126-29. Available at: http://dx.doi.org/10.3758/BF03326460. [aWM]

Goldstein, T. R. (2009) The pleasure of unadulterated sadness: Experiencing sorrow in fiction, nonfiction, and "in person." Psychology of Aesthetics Creativity, and the Arts 3(4):232-37. Available at: http://dx.doi.org/10.1037/a0015343. [aWM]

Goldstein, T. R. \& Bloom, P. (2015) Characterizing characters: How children make sense of realistic acting. Cognitive Development 34:39-50. Available at: http:// doi.org/10.1016/j.cogdev.2014.12.001. [TRG]

Goldstein, T. R. \& Filipe, A. (2017) The interpreted mind: Understanding acting. Review of General Psychology. Available at: http://dx.doi.org/10.1037/ gpr0000116. [TRG]

Goncharov, I. (1859/2009) Oblomov, trans. C. J. Hogarth. World Library Classics. $[\mathrm{rWM}]$

Goodman, N. (1968) The languages of art. Oxford University Press. [NJB]

Gordon, A. M., Stellar, J. E., Anderson, C. L., McNeil, G. D., Loew, D. \& Keltner, D. (2017) The dark side of the sublime: Distinguishing a threat-based variant of awe. Journal of Personality and Social Psychology 113(2):310-28. Available at: http://dx.doi.org/10.1037/pspp0000120. [aWM]

Gottlieb, J., Oudeyer, P.-Y., Lopes, M. \& Baranes, A. (2013) Information-seeking, curiosity, and attention: Computational and neural mechanisms. Trends in Cognitive Sciences 17(11) 585-93. Available at: https://doi.org/10.1016/j.tics 2013.09.001. [SVdC]

Grahek, N. (2007) Feeling in pain and being in pain, 2nd edition. MIT Press. [NFB]

Green, M. C. \& Brock, T. C. (2000) The role of transportation in the persuasiveness of public narratives. Journal of Personality and Social Psychology 79(5):701. [NR]

Green, M. C., Brock, T. C. \& Kaufman, G. F. (2004) Understanding media enjoyment: The role of transportation into narrative worlds. Communication Theory 14(4):311-27. [JKV]

Green, M. C., Chatham, C. \& Sestir, M. A. (2012) Emotion and transportation into fact and fiction. Scientific Study of Literature 2(1):37-59. Available at: http://dx. doi.org/10.1075/ssol.2.1.03gre. [aWM]

Green, M. C., Kass, S., Carrey, J., Herzig, B., Feeney, R. \& Sabini, J. (2008) Transportation across media: Repeated exposure to print and film. Media Psychology 11(4):512-39. Available at: http://dx.doi.org/10.1080/ 15213260802492000. [aWM]

Green, M. C., Kaufman, G., Fitzgerald, K. S., Freeman, G. \& Flanagan, M. (2017) Using narratives to raise awareness of stereotype threat in STEM. Unpublished manuscript, University at Buffalo. [MCG]

Greenson, R. R. (1953) On boredom. Journal of the American Psychoanalytic Association 1:7-21. [AE]

Grewe, O., Nagel, F., Kopiez, R. \& Altenmuller, E. (2007) Listening to music as a recreative process: Physiological, psychological, and psychoacoustical correlates of chills and strong emotions. Music Perception 24(3):297-314. Available at: http:// dx.doi.org/10.1525/mp.2007.24.3.297. [aWM]

Grodal, T. (2007) Pain, sadness, aggression, and joy: An evolutionary approach to film emotions. Projections 1(1):91-107. Available at: http://dx.doi.org/10.3167/proj. 2007.010107. [aWM]

Grodal, T. (2009) Embodied visions: Evolution, emotion, culture, and film. Oxford University Press. [KLA]

Gross, J. J. (1998) Antecedent- and response-focused emotion regulation: Divergent consequences for experience, expression, and physiology. Journal of Personality and Social Psychology 74(1):224-37. Available at: http://dx.doi.org/10.1037/ 0022-3514.74.1.224. [aWM]

Gross, J. J. \& Levenson, R. W. (1997) Hiding feelings: The acute effects of inhibiting negative and positive emotion. Journal of Abnormal Psychology 106(1):95-103. Available at: http://doi.org/10.1037/0021-843X.106.1.95. [rWM]

Gross, J. J. \& Thompson, R. A. (2006) Emotion regulation: Conceptual foundations. In: Handbook of emotion regulation, ed. J. J. Gross, pp. 1-49. Guilford. [aWM]

Grubb, E. A. (1975) Assembly line boredom and individual differences in recreation participation. Journal of Leisure Research 7(4):256-69. [AE]

Hagtvedt, H. \& Vohs, K. D. (2017) How art contributes to life's meaningfulness. Manuscript in preparation, Boston College. $[\mathrm{HH}]$

Hanfling, O. (2000) Five kinds of distance. British Journal of Aesthetics 40(1):89 102. Available at: http://dx.doi.org/10.1093/bjaesthetics/40.1.89. [SD, aWM]

Hanich, J. (2014) Judge dread: What we are afraid of when we are scared at the movies. Projections 8(2):26-49. Available at: http://dx.doi.org/10.3167/proj. 2014.080203. [aWM]
Hanich, J. \& Menninghaus, W. (2017) Beyond sadness: The multi-emotional trajectory of melodram. Cinema Journal 56(4). [aWM]

Hanich, J., Wagner, V., Shah, M., Jacobsen, T. \& Menninghaus, W. (2014) Why we like to watch sad films: The pleasure of being moved in aesthetic experiences. Psychology of Aesthetics, Creativity, and the Arts 8(2):130-43. Available at: http://doi.org/10.1037/a0035690. [GG, arWM]

Hartocollis, P. (1972) Time as a dimension of affects. Journal of the American Psychoanalytic Association 20:92-108. [AE]

Hatfield, E., Bensman, L., Thornton, P. D. \& Rapson, R. L. (2014) New perspectives on emotional contagion: A review of classic and recent research on facial mimicry and contagion. Interpersona 8(2):159. [TRG]

Hatfield, E., Cacioppo, J. \& Rapson, R. (1994) Emotional contagion. Cambridge University Press. [TRG]

Hegel, G. W. F. (1970) Vorlesungen über die Ästhetik. Suhrkamp. [aWM]

Heinen, V. K. \& Stephens, D. W. (2016) Blue jays, Cyanocitta cristata, devalue social information in uncertain environments. Animal Behaviour 112:53-62. [NR]

Hemenover, S. H. \& Schimmack, U. (2007) That's disgusting! ..., but very amusing Mixed feelings of amusement and disgust. Cognition \& Emotion 21(5):1102-13. Available at: http://dx.doi.org/10.1080/02699930601057037. [aWM]

Herrnstein, R. J. (1969) Method and theory in the study of avoidance. Psychological Review 76:49-69. [GA]

Hershfield, H. E., Scheibe, S., Sims, T. L. \& Carstensen, L. L. (2013) When feeling bad can be good: Mixed emotions benefit physical health across adulthood. Social Psychological and Personality Science 4(1):54-61. [HEH]

Hipwell, A. E., Guo, C., Phillips, M. L., Swain, J. E. \& Moses-Kolko, E. L. (2015) Right frontoinsular cortex and subcortical activity to infant cry is associated with maternal mental state talk. Journal of Neuroscience, 35(37):12725-32. [JES]

Ho, S. S., Konrath, S., Brown, S. \& Swain, J. E. (2014) Empathy and stress related neural responses in maternal decision making. Frontiers in Neuroscience 8:152. Available at: http://doi.org/10.3389/fnins.2014.00152. [JES]

Ho, S. S., \& Swain, J. E. (2017). Depression alters maternal extended amygdala response and functional connectivity during distress signals in attachment relationship. Behavioral Brain Research 325(Pt B):290-96. doi: 10.1016/j. bbr.2017.02.045 [JES]

Hodges, D. A. (2016) The neuroaesthetics of music. In: The Oxford handbook of music psychology, ed. S. Hallam, I. Cross \& M. Thaut, pp. 247-72. Oxford University Press. [EB]

Hoeken, H. \& Van Vliet, M. (2000) Suspense, curiosity, and surprise: How discourse structure influences the affective and cognitive processing of a story. Poetics 27 (4):277-86. Available at: http://dx.doi.org/10.1016/S0304-422X(99)00021-2. [aWM]

Hoemann, K., Gendron, M. \& Feldman Barrett, L. (2017) Mixed emotions in the predictive brain. Current Opinion in Behavioral Sciences 15:51-57. Available at: http://doi.org/10.1016/j.cobeha.2017.05.013. [rWM]

Höfel, L. \& Jacobsen, T. (2007) Electrophysiological indices of processing aesthetics: Spontaneous or intentional processes? International Journal of Psychophysiology 65(1):20-31. [EB]

Hoffner, C. A. \& Cantor, J. (1991) Factors affecting children's enjoyment of a frightening film sequence. Communication Monographs 58(1):41-62. Available at: http://dx.doi.org/10.1080/03637759109376213. [aWM]

Hoffner, C. A. \& Levine, K. J. (2005) Enjoyment of mediated fright and violence: A meta-analysis. Media Psychology 7(2):207-37. Available at: http://dx.doi.org/10. 1207/S1532785XMEP0702_5. [aWM]

Hogan, P. (2011) What literature teaches us about emotion. Cambridge University Press. $[\mathrm{PCH}]$

Hogan, P. (2013) How authors' minds make stories. Cambridge University Press $[\mathrm{PCH}]$

Horace (19 BCE/1932) Ars poetica [The art of poetry]. In: Horace: Satires, epistles and ars poetica, ed. \& trans. H. R. Fairclough. Heineman (current edition 1932). $[\mathrm{KO}]$

Hume, D. (1757) Of tragedy. In: D. Hume: Four dissertations, pp. 185-200. Millar. $[\mathrm{aWM}]$

Hume, D. (1757/1997) Of tragedy. In: D. Hume: Four dissertations, pp. 185-200. Thoemmes Press. [JES]

Hume, D. (1987) Of tragedy. In: Essays moral, political, and literary (revised), ed. E. F. Miller, pp. 1741-42. Liberty Classics. [SD]

Hume, D. (2004) Letter to Adam Smith, 18 July 1759. In: Adam Smith: Selected philosophical writings, ed. J. R. Otteson, pp. 243. Imprint Academic. [aWM]

Hunter, P. G. \& Schellenberg, E. G. (2010) Music and emotion. In: Music perception, ed. M. R. Jones, R. R. Fay \& A. N. Popper, pp. 129-64. Springer. [aWM]

Hunter, P. G., Schellenberg, E. G. \& Schimmack, U. (2008) Mixed affective responses to music with conflicting cues. Cognition and Emotion 22(2):327-52. Available at: http://dx.doi.org/10.1080/02699930701438145. [aWM]

Hunter, P. G., Schellenberg, E. G. \& Schimmack, U. (2010) Feelings and perceptions of happiness and sadness induced by music: Similarities, differences, and mixed emotions. Psychology of Aesthetics Creativity, and the Arts 4(1):47-56. Available at: http://dx.doi.org/10.1037/a0016873. [aWM]

Huron, D. B. (2006) Sweet anticipation: Music and the psychology of expectation. MIT Press. [aWM] 
Huron, D. B. (2011) Why is sad music pleasurable? A possible role for prolactin. Musicae Scientiae 15(2):146-58. Available at: http://dx.doi.org/10.1177/ 1029864911401171. [aWM]

Iñiguez, G., Govezensky, T., Dunbar, R., Kaski, K. \& Barrio, R. A. (2014) Effects of deception in social networks. Proceedings of the Royal Society B: Biological Sciences 281(1790):20141195. [NR]

Irvine, I. (1999) Acedia, tristitia and sloth: Early Christian forerunners to chronic ennui. Humanitas 12(1):89. [GA]

Iseminger, G. (1983) How strange a sadness? Journal of Aesthetics and Art Criticism 42(1):81-82. Available at: http://dx.doi.org/10.2307/429949. [aWM]

Ishizu, T. \& Zeki, S. (2014) A neurobiological enquiry into the origins of our experience of the sublime and beautiful. Frontiers in Human Neuroscience 8:891. Available at: https://doi.org/10.3389/fnhum.2014.00891. [GG]

Ishizu, T. \& Zeki, S. (2017) The experience of beauty derived from sorrow. Human Brain Mapping 38(8):4185-200. Available at: http://doi.org/10.1002/hbm. 23657. [GG, rWM]

Iso-Ahola, S. E. \& Weissinger, E. (1987) Leisure and boredom. Journal of Social and Clinical Psychology 5:356-64. [AE]

Ito, T. A., Larsen, J. T., Smith, N. K. \& Cacioppo, J. T. (1998) Negative information weighs more heavily on the brain: The negativity bias in evaluative categorizations. Journal of Personality and Social Psychology 75(4):887-900. Available at: http://dx.doi.org/10.1037/0022-3514.75.4.887. [aWM]

Izard, C. E. (1971) The face of emotion. Meredith. [GCC]

Jackendoff, R. \& Lerdahl, F. (2006) The capacity for music: What is it, and what's special about it? Cognition 100(1):33-72. Available at: https://doi.org/10.1016/j. cognition.2005.11.005. [SVdC]

Jacobs, A. M. (2015) Towards a neurocognitive poetics model of literary reading. In: Cognitive neuroscience of natural language use, ed. R. M. Willems, pp. 135-59. Cambridge University Press. [aWM]

Jacobsen, T. (2006) Bridging the arts and sciences: A framework for the psychology of aesthetics. Leonardo 39(2):155-62. Available at: http://dx.doi.org/10.1162/ leon.2006.39.2.155. [AC, aWM]

Jacobsen, T. \& Höfel, L. (2003) Descriptive and evaluative judgment processes: Behavioral and electrophysiological indices of processing symmetry and aesthetics. Cognitive, Affective, and Behavioral Neuroscience 3:289-99. [EB]

Jakobson, R. (1960) Linguistics and poetics. In: Style in language, ed. T. A. Sebeok, pp. 350-77. Wiley. [aWM]

Jakobson, R. (1973) Two aspects of language: Metaphor and metonymy. In: European literary theory and practice: From existential phenomenology to structuralism, ed. V. W. Gras, pp. 119-32. Dell. [arWM]

Jones, P. (2007) Ochre and rust: Artefacts and encounters on Australian frontiers. Wakefield. [NJB]

Juslin, P. N. (2013) From everyday emotions to aesthetic emotions: Towards a unified theory of musical emotions. Physics of Life Reviews 10(3):235-66. Available at: http://dx.doi.org/10.1016/j.plrev.2013.05.008. [NFB, aWM]

Kahneman, D., Fredrickson, B. L., Schreiber, C. A. \& Redelmeier, D. A. (1993) When more pain is preferred to less: Adding a better end. Psychological Science 4(6):401-405. Available at: http://dx.doi.org/10.1111/j.1467-9280.1993.tb00589. x. [aWM]

Kamber, R. (2011) Experimental philosophy of art. The Journal of Aesthetics and Art Criticism 69(2):197-208. [NR]

Kamstra, M. J., Kramer, L. A. \& Levi, M. D. (2003) Winter blues: A SAD stock market cycle. American Economic Review 93(1):324-43. [HEH]

Kant, I. (1951) Critique of judgement, trans. \& ed. J. H. Bernard. Hafner. [EB]

Kant, I. (1963/1790) Kritik der Urteilskraft [Critique of Judgment]. Philipp Reclam. Available at: http://gutenberg.spiegel.de/buch/kritik-der-urteilskraft-3507/1. [GG]

Kant, I. (1798/1996) Anthropology from a pragmatic point of view, trans. V. L. Dowdell, ed. H. H. Rudnick. Southern Illinois University Press. [aWM]

Kantor-Martynuska, J. \& Horabik, J. (2015) Granularity of emotional responses to music: The effect of musical expertise. Psychology of Aesthetics, Creativity, and the Arts 9(3):235-47. Available at: http://doi.org/10.1037/a0039107. [rWM]

Kashdan, T. B., Barrett, L. F. \& McKnight, P. E. (2015) Unpacking emotion differentiation: Transforming unpleasant experience by perceiving distinctions in negativity. Current Directions in Psychological Science 24:10-16. [NFB]

Kawakami, A. \& Katahira, K. (2015) Influence of trait empathy on the emotion evoked by sad music and on the preference for it. Frontiers in Psychology 6:1541. Available at: http://dx.doi.org/10.3389/fpsyg.2015.01541. [aWM]

Keating, P. (2011) The plot point, the darkest moment, and the answered question: Three ways of modelling the three-quarter-point. Journal of Screenwriting 2 (1):85-98. Available at: http://doi.org/10.1386/josc.2.1.85_1. [KLA]

Keen, S. (2006) A theory of narrative empathy. Narrative 14(3):207-36. Available at: http://dx.doi.org/10.1353/nar.2006.0015. [aWM]

Keller, P. E. \& Schubert, E. (2011) Cognitive and affective judgements of syncopated musical themes. Advances in Cognitive Psychology 7:142-56. Available at: http://dx.doi.org/10.2478/v10053-008-0094-0. [aWM]

Kelly, J. R., Iannone, N. E. \& McCarty, M. K. (2016) Emotional contagion of anger is automatic: An evolutionary explanation. The British Journal of Social Psychology 55(1):182-91. Available at: http://doi.org/10.1111/bjso.12134. [TRG]
Keltner, D. \& Haidt, J. (1999) Social functions of emotions at four levels of analysis. Cognition and Emotion 13:505-21. Available at: http://doi.org/10.1080/ 026999399379168. [BE]

Keltner, D. \& Haidt, J. (2003) Approaching awe, a moral, spiritual, and aesthetic emotion. Cognition and Emotion 17(2):297-314. Available at: http://dx.doi.org/ 10.1080/02699930302297. [aWM]

Kemp, G. (1999) The aesthetic attitude. British Journal of Aesthetics 39(4):392-99. [EB]

Kersten, D., Mamassian, P. \& Yuille, A. (2004) Object perception as Bayesian inference. Annual Review of Psychology 55:271-304. Available at: https://doi. org/10.1146/annurev.psych.55.090902.142005. [SVdC]

Kesner, L. (2014) The predictive mind and the experience of visual art work. Frontiers in Psychology 5:1417. [MN]

Kidd, D. C. \& Castano, E. (2013) Reading literary fiction improves theory of mind. Science 342(6156):377-80. Available at: http://dx.doi.org/10.1126/science. 1239918. [aWM]

Kim, J.-W., Kim, S.-E., Kim, J.-J., Jeong, B., Park, C.-H., Son, A., Song, J. \& Ki, S (2009) Compassionate attitude toward others' suffering activates the mesolimbic neural system. Neuropsychologia 47:2073-81. [PCH]

Kim, P., Leckman, J. F., Mayes, L. C., Feldman, R., Wang, X. \& Swain, J. E. (2010a) The plasticity of human maternal brain: Longitudinal changes in brain anatomy during the early postpartum period. Behavioral Neuroscience 124(5):695-700. [JES]

Kim, P., Leckman, J. F., Mayes, L. C., Newman, M. A., Feldman, R. \& Swain, J. E. (2010b) Perceived quality of maternal care in childhood and structure and function of mothers' brain. Developmental Science 13(4):662-73. Available at: http://doi.org/10.1111/j.1467-7687.2009.00923.x. [JES]

Kim, P., Rigo, P., Leckman, J. F., Mayes, L. C., Cole, P. M., Feldman, R. \& Swain, J. E. (2015) A prospective longitudinal study of perceived infant outcomes at 18-24 months: Neural and psychological correlates of parental thoughts and actions assessed during the first month postpartum. Frontiers in Psychology 6:1772. Available at: http://doi.org/10.3389/fpsyg.2015.01772. [JES]

Kim, P., Rigo, P., Mayes, L. C., Feldman, R., Leckman, J. F. \& Swain, J. E. (2014) Neural plasticity in fathers of human infants. Society for Neuroscience 9(5):522 35. Available at: http://doi.org/10.1080/17470919.2014.933713. [JES]

Kim, P., Strathearn, L. \& Swain, J. E. (2016) The maternal brain and its plasticity in humans. Hormones and Behavior 77:113-23. Available at: http://doi.org/10. 1016/j.yhbeh.2015.08.001. [JES]

Kinateder, B. (2011) Rührung unter der Lupe [Feeling touched under the magnifying glass]. Televizion 24:10-11. [TWS]

Kirk, U., Skov, M., Christensen, M. S. \& Nygaard, N. (2009a) Brain correlates of aesthetic expertise: A parametric fMRI study. Brain and Cognition 69:306-15. [RTA]

Kirk, U., Skov, M., Hulme, O., Christensen, M. S. \& Zeki, S. (2009b) Modulation of aesthetic value by semantic context: An fMRI study. Neuroimage 44(3):112532. Available at: http://doi.org/10.1016/j.neuroimage.2008.10.009. [NJB]

Kivy, P. (1991) Music alone: Philosophical reflections on the purely musical experience. Cornell University Press. [aWM]

Klimecki, O. M., Leiberg, S., Lamm, C. \& Singer, T. (2012) Functional neural plasticity and associated changes in positive affect after compassion training. Cerebral Cortex 23 (7): 1552-61. Available at: http://dx.doi.org/10.1093/cercor/ bhs142. [aWM]

Klimek, P., Kreuzbauer, R. \& Thurner, S. (2017) Countersignaling drives competition between cultural elites: Quantitative evidence from fashion/style cycles in music. Manuscript in preparation. [RK]

Knobloch-Westerwick, S. \& Keplinger, C. (2006) Mystery appeal: Effects of uncertainty and resolution on the enjoyment of mystery. Media Psychology 8 (3):193-212. Available at: http://doi.org/10.1207/S1532785xmep0803_1. [arWM]

Knoop, C. A., Wagner, V., Jacobsen, T. \& Menninghaus, W. (2016) Mapping the aesthetic space of literature "from below." Poetics 56:35-49. Available at: http:// doi.org/10.1016/j.poetic.2016.02.001. [rWM]

Koelsch, S. (2014) Brain correlates of music-evoked emotions. Nature Reviews Neuroscience 15(3):170-80. Available at: http://dx.doi.org/10.1038/nrn3666. $[\mathrm{aWM}]$

Konečni, V. J. (1991) Psychological aspects of the expression of anger and violence on the stage. Comparative Drama 25:215-41. [VJK]

Konečni, V. J. (2005) The aesthetic trinity: Awe, being moved, thrills. Bulletin of Psychology and the Arts 5(2):27-44. [aWM]

Konečni, V. J. (2008) Does music induce emotion? A theoretical and methodological analysis. Psychology of Aesthetics, Creativity, and the Arts 2:115-29. Available at: https://doi.org/10.1037/1931-3896.2.2.115. [VJK]

Konečni, V. J. (2013) Music, affect, method, data: Reflections on the Carroll versus Kivy debate. American Journal of Psychology 126:179-95. Available at: https:// doi.org/10.5406/amerjpsyc.126.2.0179. [VJK]

Konečni, V. J. (2015) Emotion in painting and art installations. American Journal of Psychology 128:305-22. Available at: https://doi.org/10.5406/amerjpsyc.128.3. 0305. [VJK] 
Konečni, V. J., Brown, A. \& Wanic, R. A. (2008) Comparative effects of music and recalled life-events on emotional state. Psychology of Music 36:289-308. Available at: https://doi.org/10.1177/0305735607082621. [VJK]

Konečni, V. J., Wanic, R. A. \& Brown, A. (2007) Emotional and aesthetic antecedents and consequences of music-induced thrills. The American Journal of Psychology 120(4):619-43. Available at: http://www.jstor.org/stable/20445428. [aWM]

Korsmeyer, C. (2011) Savoring disgust: The foul and the fair in aesthetics. Oxford University Press. [SD, aWM]

Kracauer, S. (1995) The mass ornament: Weimar essays, trans. T. Y. Levin. Harvard. [rWM]

Krach, S., Cohrs, J. C., de Echeverría Loebell, N. C., Kircher, T., Sommer, J., Jansen, A. \& Paulus, F. M. (2011) Your flaws are my pain: Linking empathy to vicarious embarrassment. PLoS ONE 6(4):e18675. [TRG]

Krämer, N. C. \& Witschel, T. (2010) Demystifying the sad film paradox. A critical analysis of the question of why people enjoy the reception of sad films. Paper presented at the Annual Meeting of International Communication Association, Singapore, June 2010. [aWM]

Kreibig, S. D. (2010) Autonomic nervous system activity in emotion: A review. Biological Psychology 84(3):394-421. Available at: http://dx.doi.org/10.1016/j. biopsycho.2010.03.010. [aWM]

Kreuzbauer, R. \& Cheon, B. (2015) Strategies of counterdominance: When luxury doesn't give you power. Paper presented at the Second International Conference of the Society of Consumer Psychology, Vienna, Austria. [RK]

Kreuzbauer, R. \& Keller, J. (2017) The authenticity of cultural products: A psychological perspective. Current Directions in Psychological Science. Available at: http://epubs.surrey.ac.uk/id/eprint/814077. [RK]

Kreuzbauer, R., King, D. \& Basu, S. (2015) The mind in the object-Psychological valuation of materialized human expression. Journal of Experimental Psychology: General 144(4):764. [RK]

Kringelbach, M. L. \& Berridge, K. C. (2009) Towards a functional neuroanatomy of pleasure and happiness. Trends in Cognitive Sciences 13(11):479-87. [EB]

Kuehnast, M., Wagner, V., Wassiliwizky, E., Jacobsen, T. \& Menninghaus, W. (2014) Being moved: Linguistic representation and conceptual structure. Frontiers in Psychology: Emotion Science 5:1242. Available at: http://doi.org/10.3389/fpsyg. 2014.01242. [arWM]

Kuhn, G., Olson, J. \& Raz, A. (2016) Editorial: The psychology of magic and the magic of psychology. Frontiers in Psychology 7:1-4. [JL]

Kuijpers, M. M., Hakemulder, F., Tan, E. S. \& Doicaru, M. M. (2014) Exploring absorbing reading experiences: Developing and validating a self-report scale to measure story world absorption. Scientific Study of Literature 4(1):89-122. Available at: http://dx.doi.org/10.1075/ssol.4.1.05kui. [aWM]

Kumschick, I. R., Beck, L., Eid, M., Witte, G., Klann-Delius, G., Heuser, I., Steinleiner, R. \& Menninghaus, W. (2014) Reading and feeling: The effects of a literature-based intervention designed to increase emotional competence in second and third graders. Frontiers in Psychology 5:1448. Available at: http://dx. doi.org/10.3389/fpsyg.2014.01448. [aWM]

Lacey, S., Hagtvedt, H., Patrick, V. M., Anderson, A., Stilla, R., Deshpande, G., Hu, X, Sato, J. R., Reddy, S. \& Sathian, K. (2011) Art for reward's sake: Visual art recruits the ventral striatum. NeuroImage 55:420-33. [HH]

Ladinig, O. \& Schellenberg, E. G. (2012) Liking unfamiliar music: Effects of felt emotion and individual differences. Psychology of Aesthetics, Creativity, and the Arts 6(2):146. [KF]

Lambie, J. A. \& Marcel, A. J. (2002) Consciousness and the varieties of emotion experience: A theoretical framework. Psychological Review 109(2):219-59. Available at: http://doi.org/10.1037//0033-295x.109.2.219. [rWM]

Lamont, P. (2017) A particular kind of wonder: The experience of magic past and present. Review of General Psychology 21(1):1-8. [JL]

Lange, K. (1901) Das Wesen der Kunst. Grundzüge einer realistischen Kunstlehre [The nature of art. Outline of a realistic theory of art]. Grote. [rWM]

Larsen, J. T. \& Green, J. D. (2013) Evidence for mixed feelings of happiness and sadness from brief moments in time. Cognition and Emotion 27(8):1469-77. Available at: http://doi.org/10.1080/02699931.2013.790782. [rWM]

Larsen, J. T., Hemenover, S. H., Norris, C. J. \& Cacioppo, J. T. (2003) Turning adversity to advantage: On the virtues of the coactivation of positive and negative emotions. In: A psychology of human strengths: Fundamental questions and future directions for a positive psychology, ed. L. G. Aspinwall \& U. M. Staudinger, pp. 211-25. American Psychological Association. [arWM]

Larsen, J. T. \& McGraw, A. P. (2011) Further evidence for mixed emotions. Journal of Personality and Social Psychology 100(6):1095-110. Available at: http://doi. org/10.1037/a0021846. [arWM]

Larsen, J. T., McGraw, A. P. \& Cacioppo, J. T. (2001) Can people feel happy and sad at the same time? Journal of Personality and Social Psychology 81(4):684-96. Available at: http://dx.doi.org/10.1037/0022-3514.81.4.684. [aWM]

Larsen, R. J. \& Prizmic, Z. (2008) Regulation of emotional well-being. In: The science of subjective well-being, ed. M. Eid \& R. J. Larsen, pp. 258-89. Guilford. [aWM]

Lausberg, H. (1998) Handbook of literary rhetoric: A foundation for literary study. ed. D. E. Orton \& R. D. Anderson. Brill. [aWM]
Lazarus, R. S. (1964) A laboratory approach to the dynamics of psychological stress. American Psychologist 19(6):400-11. Available at: http://dx.doi.org/10.1037/ h0041245. [aWM]

Lazarus, R. S. (1991) Progress on a cognitive-motivational-relational theory of emotion. American Psychologist 46(8):819-34. Available at: http://doi.org/10. 1037/0003-066X.46.8.819. [rWM]

Lazarus, R. S. \& Alfert, E. (1964) Short-circuiting of threat by experimentally altering cognitive appraisal. The Journal of Abnormal and Social Psychology 69 (2):195-205. Available at: http://dx.doi.org/10.1037/h0044635. [aWM]

Lazarus, R. S., Opton E., Jr., Nomikos, M. S. \& Rankin, N. O. (1965) The principle of short-circuiting of threat: Further evidence. Journal of Personality 33(4):622-35. Available at: http://dx.doi.org/10.1111/j.1467-6494.1965. tb01408.x. [aWM]

Lazarus, R. S., Speisman, J. C., Mordkoff, A. M. \& Davison, L. A. (1962) A laboratory study of psychological stress produced by a motion picture film. Psychological Monographs 76(34):1-35. Available at: http://dx.doi.org/10.1037/h0093861. $[\mathrm{aWM}]$

Leddington, J. (2016) The experience of magic. The Journal of Aesthetics and Art Criticism 74(3):253-64. [JL]

Leder, H. (2003) Familiar and fluent! Style-related processing hypotheses in aesthetic appreciation. Empirical Studies of the Arts 21(2):165-75. [HL]

Leder, H., Belke, B., Oeberst, A. \& Augustin, M. D. (2004) A model of aesthetic appreciation and aesthetic judgments. British Journal of Psychology 95(4):489508. Available at: http://dx.doi.org/10.1348/0007126042369811. [AC, HL, aWM]

Leder, H., Gerger, G., Brieber, D. \& Schwarz, N. (2014) What makes an art expert? Emotion and evaluation in art appreciation. Emotion and Cognition 28 (6):1137-47. Available at: http://doi.org/10.1080/02699931.2013.870132. [HL]

Leder, H., Gerger, G., Dressler, S. G. \& Schabmann, A. (2012) How art is appreciated. Psychology of Aesthetics Creativity and the Arts 6(1):2-10. doi: dx.doi. org/10.1037/a0026396. [HL]

Leder, H. \& Nadal, M. (2014) Ten years of a model of aesthetic appreciation and aesthetic judgments: The aesthetic episode-Developments and challenges in empirical aesthetics. British Journal of Psychology 105(4):443-64. Available at: http://doi.org/10.1111/bjop.12084. [AC, rWM, NR]

Lee, J. Y., Lindquist, K. A. \& Nam, C. S. (2017) Emotional granularity effects on event-related brain potentials during affective picture processing. Frontiers in Human Neuroscience 11:133. [NFB]

Legrand, F. D. \& Apter, M. J. (2004) Why do people perform thrilling activities? A study based on reversal theory. Psychological Reports 94(1):307-13. Available at: http://dx.doi.org/10.2466/pr0.94.1.307-313. [aWM]

Lehne, M. \& Koelsch, S. (2015) Toward a general psychological model of tension and suspense. Frontiers in Psychology 6:79. Available at: http://dx.doi.org/10.3389/ fpsyg.2015.00079. [aWM]

Leknes, S., Berna, C., Lee, M. C., Snyder, G. D., Biele, G. \& Tracey, I. (2013) The importance of context: When relative relief renders pain pleasant. Pain 154 (3):402-10. [NFB]

Leknes, S. \& Tracy, I. (2008) A common neurobiology for pain and pleasure. Nature Reviews Neuroscience 9:314-20. [NFB]

Lerner, J. S., Li, Y., Valdesolo, P. \& Kassam, K. S. (2015) Emotion and decision making. Annual Review of Psychology 66:799-823. [HEH]

Lerner, J. S., Li, Y. \& Weber, E. U. (2013) The financial costs of sadness. Psychological Science 24(1):72-79. [HEH]

Lerner, J. S. \& Tiedens, L. Z. (2006) Portrait of the angry decision maker: How appraisal tendencies shape anger's influence on cognition. Journal of Behavioral Decision Making 19:115-37. [GA]

Leslie, A. M. (1987) Pretense and representation: The origins of 'theory of mind'. Psychological Review 94:412-26. [EST]

Lessing, G. E. (1766/1984) Laocoön. An essay on the limits of painting and poetry, trans. E. A. McCormick. Johns Hopkins University Press. [aWM]

Levinson, J. (1997a) Emotion in response to art: A survey of the terrain. In: Emotion and the arts, ed. M. Hjort \& S. Laver, pp. 20-34. Oxford University Press. [aWM]

Levinson, J. (1997b) Music and negative emotion. In: Music and meaning, ed. J. Robinson, pp. 215-41. Cornell University Press. [aWM]

Levinson, J. (2006) Musical expressiveness as hearability-as-expression. In: Contemporary debates in aesthetics and the philosophy of art, ed. M. Kieran, pp. 192-206. Blackwell. [JKV]

Levinson, J., ed. (2013) Suffering art gladly: The paradox of negative emotion in art. Palgrave Macmillan. [SD]

Lindquist, K. A. \& Barrett, L. F. (2008) Emotional complexity. In: Handbook of emotions, 3rd edition, ed. M. Lewis, J. M. Haviland-Jones \& L. F. Barrett, pp. 513-30. Guilford. [NFB]

Lindquist, K. A. \& Feldman Barrett, L. (2008) Emotional complexity. In: Handbook of emotions, 3rd edition, ed. M. Lewis, J. M. Haviland-Jones \& L. F. Barrett, pp. 513-30. Guilford. [rWM]

Lindquist, K. A., Satpute, A. B., Wager, T. D., Weber, J. \& Barrett, L. F. (2016) The brain basis of positive and negative affect: Evidence from a meta-analysis of the human neuroimaging literature. Cerebral Cortex 26:1910-22. [NFB] 
Litt, M. D. (1988) Self-efficacy and perceived control: Cognitive mediators of pain tolerance. Journal of Personality and Social Psychology 54(1):149-60. Available at: http://dx.doi.org/10.1037/0022-3514.54.1.149. [aWM]

Löker, A. (1976) Film and suspense. Matbassi. [aWM]

Lord Kames, H. H. (1751/2005) Our attachment to objects of distress. In: Essays on the principles of morality and natural religion, ed. M. C. Moran, pp. 11-22. Liberty Fund. [aWM]

Lorenz, K. (1970) The enmity between generations and its probable ethological causes. Psychoanalytic Review 57:333-77. [GA]

Lundqvist, L.-O., Carlsson, F., Hilmersson, P. \& Juslin, P. N. (2009) Emotional responses to music: Experience, expression, and physiology. Psychology of Music 37:61-90. Available at: https://doi.org/10.1177/0305735607086048. [VJK, aWM]

Madrigal, R. \& Bee, C. (2005) Suspense as an experience of mixed emotions: Feelings of hope and fear while watching suspenseful commercials. Advances in Consumer Research 32(1):561-67. [aWM]

Mag Uidhir, C. (2011) The paradox of suspense realism. Journal of Aesthetics and Art Criticism 69(2):161-71. Available at: http://dx.doi.org/10.1111/j.1540-6245. 2011.01458.x. [aWM]

Magliano, J. P., Dijkstra, K. \& Zwaan, R. A. (1996) Generating predictive inferences while viewing a movie. Discourse Processes 22(3):199-224. Available at: http:// dx.doi.org/10.1080/01638539609544973. [aWM]

Malt, B. C. (1990) Features and beliefs in the mental representation of categories. Journal of Memory and Language 29(3):289-315. [NR]

Mandler, J. M. (1984) Stories, scripts, and scenes. Aspects of schema theory. Erlbaum. [NJB, aWM]

Mar, R. A. \& Oatley, K. (2008) The function of fiction is the abstraction and simulation of social experience. Perspectives on Psychological Science 3(3):173-92. [aWM, NR]

Marsh, E. J. \& Fazio, L. K. (2006) Learning errors from fiction: Difficulties in reducing reliance on fictional stories. Memory and Cognition 34(5):1140-49. [NR]

Martindale, C. (1984) The pleasures of thought: A theory of cognitive hedonics. The Journal of Mind and Behaviour 5(1):49-80. [rWM]

Martindale, C. (2001) How does the brain compute aesthetic experience? The General Psychologist 36:25-35. [MN]

Maslow, A. H., Frager, R., Fadiman, J., McReynolds, C. \& Cox, R. (1970) Motivation and personality, vol. 2. Harper \& Row. [rWM]

Maynard-Smith, J. \& Harper, D. (2003) Animal signals. Oxford. [NR]

McGraw, A. P. \& Warren, C. (2010) Benign violations: Making immoral behavior funny. Psychological Science 21(8):1141-49. Available at: http://dx.doi.org/10. 1177/0956797610376073. [aWM]

McGraw, A. P., Warren, C., Williams, L. E. \& Leonard, B. (2012) Too close for comfort, or too far to care? Finding humor in distant tragedies and close mishaps. Psychological Science 23(10):1215-23. Available at: http://dx.doi.org/ 10.1177/0956797612443831. [aWM]

McGraw, A. P., Williams, L. E. \& Warren, C. (2014) The rise and fall of humor. Psychological distance modulates humorous responses to tragedy. Social Psychological and Personality Science 5(5):566-72. Available at: http://dx.doi.org/ 10.1177/1948550613515006. [aWM]

Meinong, A. (1902/1977) Über Annahmen. In: Alexius Meinong Gesamtausgabe, vol. 4, ed. R. Haller \& R. Kindinger. Akademische Druck- und Verlagsanstalt. [aWM]

Mendelssohn, M. (1759/1991) 82. bis 84. Literaturbrief. In: Gesammelte Schriften. Jubiläumsausgabe/Band 5,1: Rezensionsartikel in 'Briefe, die neueste Literatur betreffend' (1759-1765), ed. E. J. Engel, pp. 130-37. Frommann-Holzboog. [aWM]

Mendelssohn, M. (1761/1997) Rhapsody or additions to letters on sentiments. In: Philosophical writings, trans. D. O. Dahlstrom, pp. 131-68. Cambridge University Press. [aWM]

Menninghaus, W. (1999) In praise of nonsense: Kant and Bluebeard. Stanford University Press. [aWM]

Menninghaus, W. (2003) Disgust. Theory and history of a strong sensation, trans. H. Eiland \& J. Golb. SUNY Press. [NJB, aWM]

Menninghaus, W., Bohrn, I. C., Knoop, C. A., Kotz, S. A., Schlotz, W. \& Jacobs, A. M. (2015a) Rhetorical features facilitate prosodic processing while handicapping ease of semantic comprehension. Cognition 143:48-60. Available at: http:// dx.doi.org/10.1016/j.cognition.2015.05.026. [aWM]

Menninghaus, W., Wagner, V., Hanich, J., Wassiliwizky, E., Kuehnast, M. \& Jacobsen, T. (2015b) Towards a psychological construct of being moved. PLoS ONE 10(6):e0128451. Available at: http://dx.doi.org/10.1371/journal.pone. 0128451. [arWM, TWS]

Menninghaus, W., Wagner, V., Wassiliwizky, E., Jacobsen, T. \& Knoop, C. A. (2017) The emotional and aesthetic powers of parallelistic diction. Poetics. Available at: http://doi.org/10.1016/j.poetic.2016.12.001. [arWM]

Meyer, L. B. (1961) Emotion and meaning in music. University of Chicago Press. [aWM]

Mills, J. (1993) The appeal of tragedy: An attitude interpretation. Basic and Applied Social Psychology 14(3):255-71. Available at: http://dx.doi.org/10.1207/ s15324834basp1403_1. [aWM]
Miu, A. C. \& Balteş, F. R. (2012) Empathy manipulation impacts music-induced emotions: A psychophysiological study on opera. PLoS ONE 7(1):e30618. [JKV]

Mocaiber, I., Perakakis, P., Pereira, M. G., Pinheiro, W. M., Volchan, E., de Oliveira, L. \& Vila, J. (2011a) Stimulus appraisal modulates cardiac reactivity to briefly presented mutilation pictures. International Journal of Psychophysiology 81 (3):299-304. [NR]

Mocaiber, I., Pereira, M. G., Erthal, F. S., Machado-Pinheiro, W., David, I. A., Cagy, M., Volchan, E. \& de Oliveira, L. (2010) Fact or fiction? An event-related potential study of implicit emotion regulation. Neuroscience Letters 476(2):8488. [NR]

Mocaiber, I., Sanchez, T. A., Pereira, M. G., Erthal, F. S., Joffily, M., Araujo, D. B., Volchan, E. \& de Oliveira, L. (2011b) Antecedent descriptions change brain reactivity to emotional stimuli: A functional magnetic resonance imaging study of an extrinsic and incidental reappraisal strategy. Neuroscience 193:241-48. [NR]

Moller, D. (2014) The boring. The Journal of Aesthetics and Art Criticism 72(2):18191. [AE]

Molnar-Szakacs, I. \& Overy, K. (2006) Music and mirror neurons: From motion to 'e'motion. Social Cognitive and Affective Neuroscience 1(3):235-41. [JKV]

Morreall, J. (1985) Enjoying negative emotions in fiction. Philosophy and Literature 9(1):95-103. Available at: http://dx.doi.org/10.1353/phl.1985.0118. [aWM]

Moses-Kolko, E. L., Horner, M. S., Phillips, M. L., Hipwell, A. E. \& Swain, J. E. (2014) In search of neural endophenotypes of postpartum psychopathology and disrupted maternal caregiving. Journal of Neuroendocrinology 26(10):665-84. Available at: http://doi.org/10.1111/jne.12183. [JES]

Muller, M., Höfel, L., Brattico, E. \& Jacobsen, T. (2010) Aesthetic judgments of music in experts and laypersons - An ERP study. International Journal of Psychophysiology 76(1):40-51. Available at: http://doi.org/10.1016/j.ijpsycho.2010. 02.002. [EB]

Mulligan, K. (2009) Emotions and values. In: The Oxford handbook of philosophy of emotion, ed. P. Goldie, pp. 475-500. Oxford University Press. [aWM]

Munn, C. A. (1986) Birds that "cry wolf." Nature 319:143-45. [NR]

Musch, J. \& Klauer, K. C. (2003) The psychology of evaluation: Affective processes in cognition and emotion. Erlbaum. [aWM]

Neumann, R. \& Strack, F. (2000) "Mood contagion": The automatic transfer of mood between persons. Journal of Personality and Social Psychology 79(2):211-23. Available at: http://doi.org/10.1037//0022-3514.79.2.211. [TRG]

Newman, G. E. \& Bloom, P. (2012) Art and authenticity: The importance of originals in judgments of value. Journal of Experimental Psychology: General 141 (3):558-69. Available at: http://dx.doi.org/10.1037/a0026035. [aWM]

Ng, A. H., Liu, Y., Chen, J. Z. \& Eastwood, J. D. (2015) Culture and state boredom A comparison between European Canadians and Chinese. Personality and Individual Differences 75:13-18. [AE]

Nieminen, S., Istok, E., Brattico, E., Tervaniemi, M. \& Huotilainen, M. (2011) The development of aesthetic responses to music and their underlying neural and psychological mechanisms. Cortex 47(9):1138-46. Available at: http://doi.org/ 10.1016/j.cortex.2011.05.008. [EB]

Noordewier, M. K. \& Breugelmans, S. M. (2013) On the valence of surprise. Cognition and Emotion 27(7):1326-34. Available at: http://dx.doi.org/10.1080/ 02699931.2013.777660. [aWM]

Norris, C. J., Gollan, J., Berntson, G. G. \& Cacioppo, J. T. (2010) The current status of research on the structure of evaluative space. Biological Psychology 84 (3):422-36. Available at: http://dx.doi.org/10.1016/j.biopsycho.2010.03.011. [aWM]

Nussbaum, M. C. (2008) Upheavals of thought: The intelligence of emotions. Cambridge University Press. [aWM]

Oatley, K. (1994) A taxonomy of the emotions of literary response and a theory of identification in fictional narrative. Poetics 23(1-2):53-74. Available at: http:// doi.org/10.1016/0304-422X(94)P4296-S. [rWM]

Oatley, K. (2012) The passionate muse: Exploring emotion in stories. Oxford University Press. [PCH]

Oatley, K. (2016) Fiction: Simulation of social worlds. Trends in Cognitive Sciences 20:618-28. [KO]

Obermeier, C., Kotz, S. A., Jessen, S., Raettig, T., von Koppenfels, M. \& Menninghaus, W. (2016) Aesthetic appreciation of poetry correlates with ease of processing in event-related potentials. Cognitive, Affective \& Behavioral Neuroscience 16(2):362-73. Available at: http://dx.doi.org/10.3758/s13415-0150396-x. [aWM]

Oceja, L. \& Carrera, P. (2009) Beyond a single pattern of mixed emotional experience: Sequential, prevalence, inverse, and simultaneous. European Journal of Psychological Assessment 25(1):58-67. Available at: http://doi.org/10.1027/ 1015-5759.25.1.58. [arWM]

Ochsner, K. N. \& Gross, J. J. (2005) The cognitive control of emotion. Trends in Cognitive Sciences 9(5):242-49. Available at: http://dx.doi.org/10.1016/j.tics. 2005.03.010. [aWM]

Oliver, M. B. (1993) Exploring the paradox of the enjoyment of sad films. Human Communication Research 19(3):315-42. Available at: http://dx.doi.org/10.1111/ j.1468-2958.1993.tb00304.x. [arWM] 
Oliver, M. B. \& Bartsch, A. (2010) Appreciation as audience response: Exploring entertainment gratifications beyond hedonism. Human Communication Research 36(1):53-81. Available at: http://doi.org/10.1111/j.1468-2958.2009. 01368.x. [arWM]

Oliver, M. B., Hartmann, T. \& Woolley, J. K. (2012) Elevation in response to entertainment portrayals of moral virtue. Human Communication Research 38:360-78. Available at: http://doi.org/10.1111/j.1468-2958.2012.01427.x. [MCG]

Oliver, M. B. \& Raney, A. A. (2011) Entertainment as pleasurable and meaningful Identifying hedonic and eudaimonic motivations for entertainment consumption. Journal of Communication 61(5):984-1004. [KF]

Oliver, M. B. \& Sanders, M. (2004) The appeal of horror and suspense. In: The horror film, ed. S. Price, pp. 243-59. Rutgers University Press. [aWM]

Oliver, M. B. \& Woolley, J. K. (2010) Tragic and poignant entertainment: The gratifications of meaningfulness as emotional response. In: The Routledge handbook of emotions and mass media, ed. K. Döverin, C. von Scheve \& E. A. Konijn, pp. 134-47. Routledge. [AE, arWM]

Oliver, M. B., Woolley, J., Limperos, A., Tamul, D., Bae, K. \& Freeman, M. (2009) Do "sad" people like "sad" entertainment? Meaningfulness as a mediator of affective predictors of entertainment choice and desired affective responses. Paper presented at the annual meeting of the Association for Education in Journalism and Mass Communication, Boston, MA, August 2009. $[\mathrm{aWM}]$

Oosterwijk, S., Lindquist, K. A., Adebayo, M. \& Feldman Barrett, L. (2016) The neural representation of typical and atypical experiences of negative images: Comparing fear, disgust and morbid fascination. Social Cognitive and Affective Neuroscience 11(1):11-22. Available at: http://doi.org/10.1093/scan/nsv088. [rWM]

Oppenheimer, D. M. (2008) The secret life of fluency. Trends in Cognitive Sciences 12(6):237-41. Available at: http://dx.doi.org/10.1016/j.tics.2008.02. 014. [aWM]

Packer, M. (1989) Dissolving the paradox of tragedy. Journal of Aesthetics and Art Criticism 47(3):211-19. Available at: http://dx.doi.org/10.2307/431001. [aWM]

Pandit, S. (1976) In defence of psychical distance. British Journal of Aesthetics 16:56-60. [SD]

Panksepp, J. (1995) The emotional sources of "chills" induced by music. Music perception: An Interdisciplinary Journal 13(2):171-207. Available at: http://dx. doi.org/10.2307/40285693. [aWM]

Panksepp, J. (2007) Neurologizing the psychology of affects: How appraisal-based constructivism and basic emotion theory can coexist. Perspectives on Psychological Science 2(3):281-96. [GCC]

Panksepp, J. \& Bernatzky, G. (2002) Emotional sounds and the brain: The neuroaffective foundations of musical appreciation. Behavioural Processes 60(2):13355. Available at: http://dx.doi.org/10.1016/S0376-6357(02)00080-3. [aWM]

Panksepp, J. \& Biven, L. (2012) The archaeology of mind: Neuroevolutionary origins of human emotions. Norton. [PCH]

Parsons, M. J. (1987) How we understand art: A cognitive developmental account of aesthetic experience. Cambridge University Press. [KF]

Pearce, M. T., Zaidel, D. W., Vartanian, O., Skov, M., Leder, H., Chatterjee, A. \& Nadal, M. (2016) Neuroaesthetics: The cognitive neuroscience of aesthetic experience. Perspectives on Psychological Science 11(2):265-79. [NR]

Pekrun, R., Goetz, T., Daniels, L. M., Stupnisky, R. H. \& Perry, R. P. (2010) Boredom in achievement settings: Exploring control-value antecedents and performance outcomes of a neglected emotion. Journal of Educational Psychology 102(3):531-49. [AE]

Pelowski, M. J. (2015) Tears and transformation: Feeling like crying as an indicator of insightful or "aesthetic" experience with art. Frontiers in Psychology 6:1006. [NR]

Pelowski, M., Markey, P. S., Forster, M., Gerger, G. \& Leder, H. (2017) Move me, astonish me ... delight my eyes and brain: The Vienna integrated model of top-down and bottom-up processes in art perception (VIMAP) and corresponding affective, evaluative, and neurophysiological correlates. Physics of Life Reviews 21:80-125. Available at: https://doi.org/10.1016/j. plrev.2017.02.003. [GG]

Pelowski, M., Markey, P. S., Lauring, J. O. \& Leder, H. (2016) Visualizing the impact of art: An update and comparison of current psychological models of art experience. Frontiers in Human Neuroscience 10:160. Available at: http://doi.org/10. 3339/fnhum2016.00160. [aWM, GG]

Pennebaker, J. W., Francis, M. E. \& Booth, R. J. (2001) Linguistic inquiry and word count: LIWC 2001. Erlbaum. [KO]

Penner, L., Brannick, M. T., Webb, S. \& Connell, P. (2005) Effects on volunteering of the September 11, 2001, attacks: An archival analysis. Journal of Applied Social Psychology 35(7):1333-60. [BB]

Perlovsky, L. (2007) Neural dynamic logic of consciousness: The knowledge instinct. In: Neurodynamics of cognition and consciousness, ed. L. Perlovsky \& R. Kozma, pp. 73-108. Springer. [EB]

Perlovsky, L. (2010) Musical emotions: Functions, origins, evolution. Physics Life Reviews 7(1):2-27. Available at: http://doi.org/10.1016/j.plrev.2009.11.001. [EB]
Perlovsky, L. (2014) Aesthetic emotions, what are their cognitive functions? Frontiers in Psychology 5:98. Available at: http://doi.org/10.3389/fpsyg.2014.00098. [EB]

Preston, S. D. \& De Waal, F. B. (2002) Empathy: Its ultimate and proximate bases Behavioral and Brain Sciences 25(1):1-20. [TRG]

Price, K. (1977) The truth about psychical distance. Journal of Aesthetics and Art Criticism 35:411-23. [SD]

Prieto-Pablos, J. A. (1998) The paradox of suspense. Poetics 26(2):99-113. Available at: http://dx.doi.org/10.1016/S0304-422X(98)00014-X. [aWM]

Princess Elisabeth of Bohemia \& Descartes, R. (1645/2007) The correspondence between Princess Elisabeth of Bohemia and René Descartes, trans. L. Shapiro, ed. L. Shapiro. University of Chicago Press. [aWM]

Quigley, B. M. \& Tedeschi, J. T. (1996) Mediating effects of blame attributions on feelings of anger. Personality and Social Psychology Bulletin 22(12):1280-88. [HEH]

Quintilian (1920) The Institutio oratoria of Quintilian, trans. H. E. Butler. Harvard University Press. [aWM]

Radford, C. \& Weston, M. (1975) How can we be moved by the fate of Anna Karenina? Proceedings of the Aristotelian Society 49(Suppl):67-93. [VJK]

Rafaeli, E., Rogers, G. M. \& Revelle, W. (2007) Affective synchrony: Individual differences in mixed emotions. Personality and Social Psychology Bulletin 33 (7):915-32. Available at: http://dx.doi.org/10.1177/0146167207301009. [aWM]

Raghunathan, R. \& Trope, Y. (2002) Walking the tightrope between feeling good and being accurate: Mood as a resource in processing persuasive messages. Journal of Personality and Social Psychology 83(3):510-25. [HEH]

Rawlings, D. (2003) Personality correlates of liking for 'unpleasant' paintings and photographs. Personality and Individual Differences 34(3):395-410. [KF]

Reber, R. (2016) Critical feeling: How to use feelings strategically. Cambridge University Press. [aWM]

Reber, R., Schwarz, N. \& Winkielman, P. (2004) Processing fluency and aesthetic pleasure: Is beauty in the perceiver's processing experience? Personality and Social Psychology Review 8(4):364-82. Available at: http://dx.doi.org/10.1207/ s15327957pspr0804_3. [HL, aWM, MN]

Reber, R., Winkielman, P. \& Schwarz, N. (1998) Effects of perceptual fluency on affective judgments. Psychological Science 9(1):45-48. Available at: http://dx. doi.org/10.1111/1467-9280.00008. [aWM]

Redies, C. (2007) A universal model of esthetic perception based on the sensory coding of natural stimuli. Spatial Vision 27:97-117. [AC]

Rensink, R. A. \& Kuhn, G. (2015) A framework for using magic to study the mind Frontiers in Psychology 5:1-14. [JL]

Reybrouck, M. \& Brattico, E. (2015) Neuroplasticity beyond sounds: Neural adaptations following long-term musical aesthetic experiences. Brain Sciences 5 (1):69-91. Available at: http://doi.org/10.3390/brainsci5010069. [EB]

Rhue, J. W. \& Lynn, S. J. (1987) Fantasy proneness: The ability to hallucinate "as real as real." British Journal of Experimental and Clinical Hypnosis 4:173-80. [GA]

Rickard, N. S. (2004) Intense emotional responses to music: A test of the physiological arousal hypothesis. Psychology of Music 32(4):371-88. Available at: http://dx.doi.org/10.1177/0305735604046096. [aWM]

Riediger, M., Schmiedek, F., Wagner, G. G. \& Lindenberger, U. (2009) Seeking pleasure and seeking pain: Differences in prohedonic and contra-hedonic motivation from adolescence to old age. Psychological Science 20(12):1529-35. Available at: http://dx.doi.org/10.1111/j.1467-9280.2009.02473.x. [aWM]

Rimé, B. (2007) Interpersonal emotion regulation. In: Handbook of emotion regulation, ed. J. J. Gross, pp. 466-85. Guilford. [BE]

Rimé, B. (2009) Emotion elicits the social sharing of emotion: Theory and empirical review. Emotion Review 1:60-85. Available at: http://doi.org/10.1177/ 1754073908097189. [BE]

Rimé, B., Delfosse, C. \& Corsini, S. (2005) Emotional fascination: Responses to viewing pictures of September 11 attacks. Cognition and Emotion 19(6):92332. Available at: http://doi.org/10.1080/02699930541000039. [arWM]

Robinson, J. (2009) Aesthetic emotions (philosophical perspectives). In: The Oxford companion to emotion and the affective sciences, ed. D. Sander \& K. Scherer, pp. 6-9. Oxford University Press. [PCH]

Robinson, T., Callahan, C. \& Evans, K. (2014) Why do we keep going back? A Q method analysis of our attraction to horror movies. Operant Subjectivity 37(1/ 2):41-57. Available at: http://dx.doi.org/10.15133/j.os.2014.004. [aWM]

Routledge, C., Arndt, J., Wildschut, T., Sedikides, C., Hart, C. M., Juhl, J., Vingerhoets, A. J. J. M. \& Schlotz, W. (2011) The past makes the present meaningful: Nostalgia as an existential resource. Journal of Personality and Social Psychology 101(3):638-52. Available at: http://dx.doi.org/10.1037/a0024292. [aWM]

Routledge, C., Wildschut, T., Sedikides, C., Juhl, J. \& Arndt, J. (2012) The power of the past: Nostalgia as a meaning-making resource. Memory 20(5):452-60. [AE]

Rozin, A., Rozin, P. \& Goldberg, E. (2004) The feeling of music past: How listeners remember musical affect. Music Perception: An Interdisciplinary Journal 22 (1):15-39. Available at: http://dx.doi.org/10.1525/mp.2004.22.1.15. [aWM]

Rozin, P. (1999) Preadaptation and the puzzles and properties of pleasure. In: Wellbeing: The foundations of hedonic psychology, ed. D. Kahneman, E. Diener \& N. Schwarz, pp. 109-33. Sage. [aWM] 
Rozin, P. \& Fallon, A. E. (1987) A perspective on disgust. Psychological Review 94 (1):23-41. Available at: http://dx.doi.org/10.1037/0033-295X.94.1.23. [aWM]

Rozin, P., Guillot, L., Fincher, K., Rozin, A. \& Tsukayama, E. (2013) Glad to be sad, and other examples of benign masochism. Judgment and Decision Making 8 (4):439. [aWM]

Rozin, P. \& Haidt, J. (2013) The domains of disgust and their origins: Contrasting biological and cultural evolutionary accounts. Trends in Cognitive Sciences 17 (8):367-68. Available at: http://dx.doi.org/10.1016/j.tics.2013.06.001. [aWM]

Rozin, P., Haidt, J. \& McCauley, C. R. (2008) Disgust. In: Handbook of emotions 3rd edition, ed. M. Lewis, J. M. Haviland-Jones \& L. F. Barrett, pp. 757-76. Guilford. [aWM]

Rozin, P. \& Royzman, E. B. (2001) Negativity bias, negativity dominance, and contagion. Personality and Social Psychology Review 5(4):296-320. Available at: http://dx.doi.org/10.1207/S15327957PSPR0504_2. [aWM]

Russell, J. A. (2003) Core affect and the psychological construction of emotion. Psychological Review 110(1):145-72. Available at: http://dx.doi.org/10.1037/ 0033-295X.110.1.145. [aWM]

Russell, J. A. \& Barrett, L. F. (1999) Core affect, prototypical emotional episodes, and other things called emotion: Dissecting the elephant. Journal of Personality and Social Psychology 76(5):805-19. [GCC]

Sachs, M. E., Damasio, A. \& Habibi, A. (2015) The pleasures of sad music: A systematic review. Frontiers in Human Neuroscience 9:404. Available at: http://doi. org/10.3389/fnhum.2015.00404. [NFB, aWM, MN]

Salimpoor, V. N., Benovoy, M., Larcher, K., Dagher, A. \& Zatorre, R. J. (2011) Anatomically distinct dopamine release during anticipation and experience of peak emotion to music. Nature Neuroscience 14(2):257-62. Available at: http:// dx.doi.org/10.1038/nn.2726. [aWM]

Salimpoor, V. N., Benovoy, M., Longo, G., Cooperstock, J. R. \& Zatorre, R. J. (2009) The rewarding aspects of music listening are related to degree of emotional arousal. PLoS ONE 4(10):e7487. Available at: http://dx.doi.org/10.1371/journal. pone.0007487. [aWM]

Salimpoor, V. N., van den Bosch, I., Kovacevic, N., McIntosh, A. R., Dagher, A. \& Zatorre, R. J. (2013) Interactions between the nucleus accumbens and auditory cortices predict music reward value. Science 340:216-19. [RTA]

Salimpoor, V. N., Zald, D. H., Zatorre, R., Dagher, A. \& McIntosh, A. R. (2015) Predictions and the brain: How musical sounds become rewarding. Trends in Cognitive Sciences 19:86-91. [RTA]

Scalise Sugiyama, M. (2005) Reverse-engineering narrative: Evidence of special design. In: The literary animal, ed. J. Gottshall \& D. S. Wilson, pp. 177-96. Northwestern University Press. [aWM]

Schäfer, T., Zimmermann, D. \& Sedlmeier, P. (2014) How we remember the emotional intensity of past musical experiences. Frontiers in Psychology 5:911. Available at: http://dx.doi.org/10.3389/fpsyg.2014.00911. [aWM]

Schaller, M. (1993) Feeling bad to feel good: Comments and observations. Basic and Applied Social Psychology 14(3):285-94. Available at: http://dx.doi.org/10.1207/ s15324834basp1403_3. [aWM]

Scheff, T. J. (1979) Catharsis in healing, ritual, and drama. University of California Press. [aWM]

Schellenberg, E. G., Peretz, I. \& Vieillard, S. (2008) Liking for happy- and sadsounding music: Effects of exposure. Cognition and Emotion 22(2):218-37. Available at: http://dx.doi.org/10.1080/02699930701350753. [aWM]

Scherer, K. R. (2001) Appraisal considered as a process of multi-level sequential checking. In: Appraisal processes in emotion: Theory, methods, research, ed. K. R. Scherer, A. Schorr, \& T. Johnstone, pp. 92-120. Oxford University Press. [KLA]

Scherer, K. R. (2005) What are emotions? And how can they be measured? Social Science Information - Sur Les Sciences Sociales 44(4):695-729. Available at: http://doi.org/10.1177/0539018405058216. [arWM]

Scherer, K. R. (2012) Emotion in action, interaction, music and speech. In: Language, music and the brain: A mysterious relationship, ed. M. Arbib, pp. 10740. MIT Press. [aWM]

Scherer, K. R., Dan, E. S. \& Flykt, A. (2006) What determines a feeling's position in affective space? A case for appraisal. Cognition \& Emotion 20(1):92-113. Available at: http://dx.doi.org/10.1080/02699930500305016. [aWM]

Schimmack, U. (2001) Pleasure, displeasure, and mixed feelings: Are semantic opposites mutually exclusive? Cognition and Emotion 15(1):81-97. Available at: http://dx.doi.org/10.1080/02699930126097. [aWM]

Schindler, I., Hosoya, G., Menninghaus, W., Beermann, U., Wagner, V., Eid, M. \& Scherer, K. R. (2017) Measuring aesthetic emotions: A review of the literature and a new assessment tool. PLoS ONE 12(6):e0178899. Available at: http://doi. org/10.1371/journal.pone.0178899. [rWM]

Schmidt-Atzert, L. \& Ströhm, W. (1983) Ein Beitrag zur Taxonomie der Emotionswörter [A contribution to the taxonomy of emotion words]. Psychologische Beiträge 25(1):126-41. [TWS]

Schneider, H. J. (1995) The staging of the gaze: Aesthetic illusion and the scene of nature in the eighteenth century. In: Reflecting senses: Perception and appearance in literature, culture, and the arts, ed. W. Pape \& F. Burwick, pp. 77-95. Walter de Gruyter. [GCC]
Schneider, I.K., \& Schwarz, N. (2017). Mixed feelings: The case of ambivalence Current Opinion in Behavioral Sciences 15:39-45. DOI: 10.1016/j. cobeha.2017.05.012. [HL]

Schneider, I. K., Veenstra, L., van Harreveld, F., Schwarz, N. \& Koole, S. (2016) Let's not be indifferent about neutrality: Neutral ratings in the IAPS mask mixed affective responses. Emotion 16:426-30. Available at: http://doi.org/10. 1037/emo0000164. [HL]

Schramm, H. \& Wirth, W. (2010) Exploring the paradox of sad-film enjoyment: The role of multiple appraisals and meta-appraisals. Poetics 38(3):319-35. Available at: http://dx.doi.org/10.1016/j.poetic.2010.03.002. [aWM]

Schubert, E. (2013) Loved music can make a listener feel negative emotions Musicae Scientiae 17(1):11-26. Available at: http://dx.doi.org/10.1177/ 1029864912461321. [aWM]

Schubert, E. (2016) Enjoying sad music: Paradox or parallel processes? Frontiers in Human Neuroscience 10:312. Available at: http://dx.doi.org/10.3389/fnhum. 2016.00312. [aWM]

Schubert, T. W., Zickfeld, J. H., Seibt, B. \& Fiske, A. P. (2016) Moment-to-moment changes in feeling moved match changes in closeness, tears, goosebumps, and warmth: Time series analyses. Cognition and Emotion. Available at: http://doi. org/10.1080/02699931.2016.1268998. [rWM, TWS]

Sedikides, C., Wildschut, T., Arndt, J. \& Routledge, C. (2008) Nostalgia: Past, present, and future. Current Directions in Psychological Science 17(5):304-307. Available at: http://dx.doi.org/10.1111/j.1467-8721.2008.00595.x. [AE, aWM]

Seibt, B., Schubert, T. W., Zickfeld, J. H. \& Fiske, A. P. (2017) Interpersonal closeness and morality predict feelings of being moved. Emotion 17(3):389-94. Available at: http://dx.doi.org/10.1037/emo0000271. [TWS]

Shaw, D. (2001) Power, horror and ambivalence. Film and Philosophy (Special Edition on Horror), 1-13. [aWM]

Shuman, V., Sander, D. \& Scherer, K. R. (2013) Levels of valence. Frontiers in Psychology 4:261. Available at: http://doi.org/10.3389/fpsyg.2013.00261. [rWM]

Silvia, P. J. (2005a) What is interesting? Exploring the appraisal structure of interest Emotion 5(1):89-102. Available at: http://doi.org/10.1037/1528-3542.5.1.89. $[\mathrm{rWM}]$

Silvia, P. J. (2005b) Emotional responses to art: From collation and arousal to cognition and emotion. Review of General Psychology 9(4):342. Available at: https:// pdfs.semanticscholar.org/a87a/95da82793dfe51b6f0a6d9d52c1c5d428f7c.pdf. $[\mathrm{SVdC}]$

Silvia, P. J. (2008) Interest - The curious emotion. Current Directions in Psychological Science 17(1): 57-60. [HH]

Silvia, P. J. (2009) Looking past pleasure: Anger, confusion, disgust, pride, surprise, and other unusual aesthetic emotions. Psychology of Aesthetics, Creativity, and the Arts 3(1):48-51. Available at: http://dx.doi.org/ 10.1037/a0014632. [aWM, $\mathrm{MN}]$

Silvia, P. J. (2010) Confusion and interest: The role of knowledge emotions in aesthetic experience. Psychology of Aesthetics, Creativity, and the Arts 4(2):75-80. Available at: http://dx.doi.org/10.1037/a0017081. [arWM]

Silvia, P. J. (2012) Human emotions and aesthetic experience: An overview of empirical aesthetics. In: Aesthetic science: Connecting minds, brains, and experience, ed. A. Shimamura \& S. E. Palmer, pp. 250-75. Oxford University Press. [MN]

Silvia, P. J. \& Brown, E. M. (2007) Anger, disgust, and the negative aesthetic emotions: Expanding an appraisal model of aesthetic experience. Psychology of Aesthetics, Creativity, and the Arts 1(2):100-106. [EST]

Silvia, P. J., Fayn, K., Nusbaum, E. C. \& Beaty, R. E. (2015) Openness to experience and awe in response to nature and music: Personality and profound aesthetic experiences. Psychology of Aesthetics, Creativity, and the Arts 9(4):376-84. Available at: http://dx.doi.org/10.1037/aca0000028. [KF, aWM]

Silvia, P. J. \& Nusbaum, E. C. (2011) On personality and piloerection: Individual differences in aesthetic chills and other unusual aesthetic experiences. Psychology of Aesthetics, Creativity, and the Arts 5(3):208-14. [KF]

Singer, T. \& Lamm, C. (2009) The social neuroscience of empathy. Annals of the New York Academy of Sciences 1156(1):81-96. Available at: http://dx.doi.org/10. 1111/j.1749-6632.2009.04418.x. [GG, aWM]

Singer, T., Seymour, B., O’Doherty, J., Kaube, H., Dolan, R. J. \& Frith, C. D. (2004) Empathy for pain involves the affective but not sensory components of pain. Science 303(5661):1157-62. Available at: http://dx.doi.org/10.1126/science. 1093535. [aWM]

Skulsky, H. (1980) On being moved by fiction. Journal of Aesthetics and Art Criticism 39(1):5-14. Available at: http://dx.doi.org/10.2307/429914. [aWM]

Slater, M. D. (2002) Entertainment education and the persuasive impact of narratives. In: Narrative impact: Social and cognitive foundations, ed. M. C. Green, J. J. Strange \& T. C. Brock. pp. 157-81. Erlbaum. [MCG]

Sloman, S. A. \& Lagnado, D. (2015) Causality in thought. Annual Review of Psychology 66:22:3-47. [NR]

Smidt, K. E. \& Suvak, M. K. (2015) A brief, but nuanced, review of emotional granularity and emotion differentiation research. Current Opinion in Psychology 3:48-51. Available at: http://doi.org/10.1016/j.copsyc.2015.02.007. [NFB, $\mathrm{rWM}]$ 
Smith, J. K. (2014) The museum effect. Rowman \& Littlefield. [MN]

Smith, L. F., Smith, J. K. \& Tinio, P. P. L. (2017) Time spent viewing art and reading labels. Psychology of Aesthetics, Creativity, and the Arts 11(1):77-85. Available at: http://doi.org/10.1037/aca0000049. [AC]

Smuts, A. (2009a) Art and negative affect. Philosophy Compass 4(1):39-55. Available at: http://dx.doi.org/10.1111/j.1747-9991.2008.00199.x. [aWM]

Smuts, A. (2009b) The paradox of suspense. Stanford Encyclopedia of Philosophy. 2009. Available at: http://plato.stanford.edu/entries/paradox-suspense/. [aWM]

Solomon, R. C. (2003) I. Emotions, thoughts and feelings: What is a 'cognitive theory' of the emotions and does it neglect affectivity? Royal Institute of Philosophy Supplements 52:1-18. Available at: http://dx.doi.org/10.1017/ S1358246100007864. [aWM]

Solomon, R. L. (1980) The opponent-process theory of acquired motivation. The costs of pleasure and benefits of pain. American Psychologist 35(8):691-712. Available at: http://dx.doi.org/10.1037/0003-066X.35.8.691. [aWM]

Solomon, R. L. \& Corbit, J. D. (1974) An opponent-process theory of motivation: I. Temporal dynamics of affect. Psychological Review 81(2):119-45. Available at: http://dx.doi.org/10.1037/h0036128. [aWM]

Sparks, G. G. (1991) The relationship between distress and delight in males' and females' reactions to frightening films. Human Communication Research 17 (4):625-37. Available at: http://dx.doi.org/10.1111/j.1468-2958.1991.tb00247.x. [aWM, JKV]

Sparks, G. G. \& Ogles, R. M. (1994) The role of preferred coping style and emotional forewarning in predicting emotional reactions to a suspenseful film. Communication Reports 7(1):1-10. Available at: http://dx.doi.org/10.1080/ 08934219409367577. [aWM]

Sripada, C., Swain, J. D., Ho, S. S. \& Swain, J. E. (2014) Automatic goals and conscious regulation in social cognitive affective neuroscience. Behavioral and Brain Sciences 37(2):156-57. Available at: http://doi.org/10.1017/ S0140525X13002161. [JES]

Stanislavsky, K. (1989) An actor prepares. Theatre Arts Book/Routledge. [TRG]

Stel, M., van Baaren, R. B. \& Vonk, R. (2008) Effects of mimicking: Acting prosocially by being emotionally moved. European Journal of Social Psychology 38 (6):965-76. Available at: http://dx.doi.org/10.1002/ejsp.472. [aWM]

Stolnitz, J. (1961) On the origins of "aesthetic disinterestedness." The Journal of Aesthetics and Art Criticism 20(2):131-43. [NR]

Strange, J. J. \& Leung, C. C. (1999) How anecdotal accounts in news and in fiction can influence judgments of a social problem's urgency, causes, and cures. Personality and Social Psychology Bulletin 25(4):436-49. [NR]

Sumpf, M., Jentschke, S. \& Koelsch, S. (2015) Effects of aesthetic chills on a cardiac signature of emotionality. PLOS ONE 10(6):e0130117. Available at: http://dx. doi.org/10.1371/journal.pone.0130117. [aWM]

Sundberg, N. D., Latkin, C. A., Farmer, R. F. \& Saoud, J. (1991) Boredom in young adults: Gender and cultural comparisons. Journal of Cross-Cultural Psychology 22(2):209-23. [AE]

Swain, J. E., Dayton, C. J., Kim, P., Tolman, R. M. \& Volling, B. L. (2014) Progress on the paternal brain: Theory, animal models, human brain research, and mental health implications. Infant Mental Health Journal 35(5):394-408. Available at: http://doi.org/10.1002/imhj.21471. [JES]

Swain, J. E. \& Ho, S. S. (2017a) Neuroendocrine mechanisms for parental sensitivity: Overview, recent advances and future directions. Current Opinion in Psychology 15(1):105-10. [JES]

Swain, J. E. \& Ho, S. S. (2017b) Parental brain: The crucible of compassion. In: The Oxford handbook of compassion science, ed. E. M. Seppala, E. Simon-Thomas, S. L. Brown, M. C. Worline, C. D. Cameron, J. R. Doty \& J. R. Doty, pp. 65-78. Oxford University Press. [JES]

Swain, J. E., Ho, S. S., Rosenblum, K. L., Morelen, D., Dayton, C. J. \& Muzik, M. (2017) Parent-child intervention decreases stress and increases maternal brain activity and connectivity during own baby-cry: An exploratory study. Development and Psychopathology 29(2):535-53. Available at: http://doi.org/10.1017/ S0954579417000165. [JES]

Swain, J. E., Kim, P., Spicer, J., Ho, S. S., Dayton, C. J., Elmadih, A. \& Abel, K. M (2014) Approaching the biology of human parental attachment: Brain imaging, oxytocin and coordinated assessments of mothers and fathers. Brain Research 1580:78-101. Available at: http://doi.org/10.1016/j.brainres.2014.03. 007. [JES]

Swain, J. E., Konrath, S., Brown, S. L., Finegood, E. D., Akce, L. B., Dayton, C. J. \& Ho, S. S. (2012) Parenting and beyond: Common neurocircuits underlying parental and altruistic caregiving. Parenting, Science, and Practice 12(2-3):11523. Available at: http://doi.org/10.1080/15295192.2012.680409. [JES]

Swain, J. E. \& Lorberbaum, J. P. (2008) Imaging the human parental brain. Neurobiology of the Parental Brain 6:83-100. Academic Press. Available at: http:// doi.org/10.1016/B978-0-12-374285-8.00006-8. [JES]

Swain, J. E., Mayes, L. C. \& Leckman, J. F. (2004) The development of parent-infant attachment through dynamic and interactive signaling loops of care and cry. Behavioral and Brain Sciences 27(4):472-73. [JES]

Swami, V. (2013) Context matters: Investigating the impact of contextual information on aesthetic appreciation of paintings by Max Ernst and Pablo Picasso.
Psychology of Aesthetics, Creativity, and the Arts 7(3):285-95. Available at: http://doi.org/10.1037/a0030965. [NJB]

Swiss, J. I. (2002) Why magic sucks. In: Shattering illusions, pp. 3-12. Hermetic. Available at: http://www.honestliar.com/fm/works/whymagicsucks.html. [JL]

Takahashi, S. (1995) Aesthetic properties of pictorial perception. Psychological Review 102(4):671-83. [NJB]

Tal-Or, N. \& Cohen, J. (2010) Understanding audience involvement: Conceptualizing and manipulating identification and transportation. Poetics 38(4):402-18. [JKV]

Tamborini, R. \& Stiff, J. (1987) Predictors of horror film attendance and appeal. An analysis of the audience for frightening films. Communication Research 14 (4):415-36. Available at: http://dx.doi.org/10.1177/009365087014004003. [KF, aWM]

Tamborini, R., Stiff, J. \& Heidel, C. (1990) Reacting to graphic horror. A model of empathy and emotional behavior. Communication Research 17(5):616-40. Available at: http://dx.doi.org/10.1177/009365090017005003. [aWM, JKV]

Tan, E. S. H. (2000) Emotion, art, and the humanities. In: Handbook of emotions, 2nd edition, vol. 3, ed. M. D. Lewis, pp. 116-34. Guilford. [rWM]

Tan, E. S. H. (2008) Entertainment is emotion: The functional architecture of the entertainment experience. Media Psychology 11(1):28-51. Available at: http:// dx.doi.org/10.1080/15213260701853161. [aWM]

Tan, E. S. H. (2009) Being moved. In: The Oxford companion to emotion and the affective sciences, ed. D. Sander \& K. R. Scherer. Oxford University Press. [aWM]

Taruffi, L. \& Koelsch, S. (2014) The paradox of music-evoked sadness: An online survey. PLoS ONE 9(10):e110490. Available at: http://dx.doi.org/10.1371/ journal.pone.0110490. [NFB, aWM]

Taylor, S. E., Klein, L. C., Lewis, B. P., Gruenewald, T. L., Gurung, R. A. \& Updegraff, J. A. (2000) Biobehavioral responses to stress in females: Tend-andbefriend, not fight-or-flight. Psychological Review 107(3):411-29. [BB]

Tenore, M. (2014) Restorative narratives: Defining a new strength-based genre. Retrieved: November 16, 2015. Available at: http://ivoh.org/restorativenarrative/. [MCG]

Tetlock, P. E., Bernzweig, J. \& Gallant, J. L. (1985) Supreme Court decision making: Cognitive style as a predictor of ideological consistency of voting. Journal of Personality and Social Psychology 48:1227-39. [HH]

Tinio, P. P. L. (2013) From artistic creation to aesthetic reception: The mirror model of art. Psychology of Aesthetics, Creativity, and the Arts 7(3):265-75. Available at: http://doi.org/10.1037/a0030872. [AC, MN]

Tinio, P. P. L. \& Leder, H. (2009) Just how stable are stable aesthetic features? Symmetry, complexity, and the jaws of massive familiarization. Acta Psychologica 130(3):241-50. Available at: http://dx.doi.org/10.1016/j.actpsy.2009.01. 001. [aWM]

Tinio, P. P. L., Leder, H. \& Strasser, M. (2011) Image quality and the aesthetic judgment of photographs: Contrast, sharpness, and grain teased apart and put together. Psychology of Aesthetics Creativity, and the Arts 5(2):165-76. Available at: http://dx.doi.org/10.1037/a0019542. [aWM]

Tokaji, A. (2003) Research for determinant factors and features of emotional responses of "kandoh" (the state of being emotionally moved). Japanese Psychological Research 45(4):235-49. Available at: http://dx.doi.org/10.1111/14685884.00226. [arWM]

Tomkins, S. S. (1978) Script theory: Differential magnification of affects. Nebraska Symposium on Motivation 26:201-36. [GA]

Tooby, J. \& Cosmides, L. (2001) Does beauty build adapted minds? Toward an evolutionary theory of aesthetics, fiction, and the arts. SubStance 30(1):6-27. [NR]

Trehub, S. E. (2000) Human processing predispositions and musical universals. In: The origins of music, ed. N. L. Wallin, B. Merker \& S. Brown, pp. 427-48. MIT Press. [aWM]

Trope, Y., Ferguson, M. \& Raghunathan, R. (2000) Mood as a resource in processing self-relevant information. In: Handbook of affect and cognition, vol. 1, ed. J. P. Forgas, pp. 258-76. Erlbaum. [HEH]

Trope, Y. \& Liberman, N. (2010) Construal-level theory of psychological distance. Psychological Review 117(2):440-63. Available at: http://dx.doi.org/10.1037/ a0018963. [aWM]

Trope, Y., Liberman, N. \& Wakslak, C. (2007) Construal levels and psychological distance: Effects on representation, prediction, evaluation, and behavior. Journal of Consumer Psychology 17(2):83-95. Available at: http://dx.doi.org/10. 1016/S1057-7408(07)70013-X. [aWM]

Trope, Y. \& Neter, E. (1994) Reconciling competing motives in self-evaluation: The role of self-control in feedback seeking. Journal of Personality and Social Psychology 66(4):646-57. [HEH]

Turner, S. A., Jr. \& Silvia, P. J. (2006) Must interesting things be pleasant? A test of competing appraisal structures. Emotion 6(4):670-74. [KF]

Tybur, J. M., Lieberman, D., Kurzban, R. \& DeScioli, P. (2013) Disgust: Evolved function and structure. Psychological Review 120(1):65-84. Available at: http:// dx.doi.org/10.1037/a0030778. [aWM]

Vaish, A., Grossmann, T. \& Woodward, A. (2008) Not all emotions are created equal The negativity bias in social-emotional development. Psychological Bulletin 134 (3):383-403. Available at: http://dx.doi.org/10.1037/0033-2909.134.3.383. [aWM] 
Van de Cruys, S. (2017) Affective value in the predictive mind. In: Philosophy and predictive processing, ed. T. K. Metzinger \& W. Wiese. MIND Group. Available at: https://doi.org/10.15502/9783958573253. [SVdC]

Van de Cruys, S. \& Wagemans, J. (2011) Putting reward in art: A tentative prediction error account of visual art. Iperception 2(9):1035-62. Available at: http://doi.org/ 10.1068/i0466aap. [RTA, rWM, MN, SVdC]

Van Dongen, N. N., Van Strien, J. W. \& Dijkstra, K. (2016) Implicit emotion regulation in the context of viewing artworks: ERP evidence in response to pleasant and unpleasant pictures. Brain and Cognition 107:48-54. Available at: http://dx. doi.org/10.1016/j.bandc.2016.06.003. [aWM, NR]

van Laer, T., de Ruyter, K., Visconti, L. M. \& Wetzels, M. (2014) The extended transportation-imagery model: A meta-analysis of the antecedents and consequences of consumers' narrative transportation. Journal of Consumer Research 40(5):797-817. [MCG]

Van Tilburg, W. A. \& Igou, E. R. (2011) On boredom and social identity: A pragmatic meaning-regulation approach. Personality and Social Psychology Bulletin 37:1679-91. [AE]

Van Tilburg, W. A. \& Igou, E. R. (2012) On boredom: Lack of challenge and meaning as distinct boredom experiences. Motivation and Emotion 36(2):18194. [AE]

Van Tilburg, W. A., Igou, E. R. \& Sedikides, C. (2013) In search of meaningfulness: Nostalgia as an antidote to boredom. Emotion 13(3):450-61. [AE]

Vanhaerren, M., d'Errico, F., Stronger, C., James, S. L., Todd, J. A. \& Mienis, H. K. (2006) Middle palaeolithic shell beads in Israel and Algeria. Science 312:1785. [KO]

Vartanian, O. \& Goel, V. (2004) Neuroanatomical correlates of aesthetic preference for paintings. Neuroreport 15(5):893-97. [AC]

Visch, V. \& Tan, E. (2007) Effect of film velocity on genre recognition. Media Psychology 9(1):59-75. Available at: http://dx.doi.org/10.1080/ 15213260709336803. [aWM]

Visch, V. \& Tan, E. (2009) Categorizing moving objects into film genres: The effect of animacy attribution, emotional response, and the deviation from non-fiction. Cognition 110:265-72. [EST]

Visch, V. \& Tan, E. S. H. (2008) Narrative versus style: Effect of genre-typical events versus genre-typical filmic realizations on film viewers' genre recognition. Poetics 36(4):301-15. Available at: http://dx.doi.org/10.1016/j.poetic.2008.03.003. [aWM]

Visch, V., Tan, E. S. H. \& Molenaar, D. (2010) The emotional and cognitive effect of immersion in film viewing. Cognition and Emotion 24(8):1439-45. Available at: http://dx.doi.org/10.1080/02699930903498186. [arWM]

Visch, V. T., Tan, E. S. \& Saakes, D. P. (2015) Viewer knowledge: Application of exposure-based layperson knowledge in genre-specific animation production. International Journal of Design 9(1):83-89. [EST]

Vodanovich, S. J. (2003) Psychometric measures of boredom: A review of the literature. The Journal of Psychology 137(6):569-95. [AE]

Vodanovich, S. J. \& Watt, J. D (2015) Self-report measures of boredom: An updated review of the literature. The Journal of Psychology 150(2):196-228. Available at: http://doi.org/10.1080/00223980.2015.1074531. [AE]

von Dawans, B., Fischacher, U., Kirschbaum, C., Fehr, E. \& Heinrichs, M. (2012) The social dimension of stress reactivity: Acute stress increases prosocial behavior in humans. Psychological Science 23:651-60. [BB]

Vuoskoski, J. K. \& Eerola, T. (2012) Can sad music really make you sad? Indirect measures of affective states induced by music and autobiographical memories Psychology of Aesthetics, Creativity, and the Arts 6(3):204-13. Available at: http://dx.doi.org/10.1037/a0026937. [aWM]

Vuoskoski, J. K. \& Eerola, T. (2015) Extramusical information contributes to emotions induced by music. Psychology of Music 43(2):262-74. [JKV]

Vuoskoski, J. K. \& Eerola, T. (2017) The pleasure evoked by sad music is mediated by feelings of being moved. Frontiers in Psychology 8:439. Available at: http:// doi.org/10.3389/fpsyg.2017.00439. [NFB, rWM, JKV]

Vuoskoski, J. K., Thompson, W. F., McIlwain, D. \& Eerola, T. (2012) Who enjoys listening to sad music and why? Music Perception: An Interdisciplinary Journal 29(3):311-17. [NFB, KF, JKV]

Wagner, V., Klein, J., Hanich, J., Shah, M., Menninghaus, W. \& Jacobsen, T. (2016) Anger framed: A field study on emotion, pleasure, and art. Psychology of Aesthetics, Creativity, and the Arts 10(2):134-46. Available at: https://doi.org/10. 1037/aca0000029. [VJK, aWM]

Wagner, V., Menninghaus, W., Hanich, J. \& Jacobsen, T. (2014) Art schema effects on affective experience: The case of disgusting images. Psychology of Aesthetics, Creativity, and the Arts 8(2):120-29. Available at: http://dx.doi.org/10.1037/ a0036126. [arWM, MN, NR]

Walton, K. L. (1970) Categories of art. The Philosophical Review 79(3):334-67. Available at: http://dx.doi.org/10.2307/2183933. [NJB, aWM]

Walton, K. L. (1990) Mimesis as make-believe: On the foundations of the representational arts. Harvard University Press. [aWM]

Wänke, M. \& Hansen, J. (2015) Relative processing fluency. Current Directions in Psychological Science 24(3):195-99. Available at: https://doi.org/10.1177/ 0963721414561766. [SVdC]

Wassiliwizky, E., Jacobsen, T., Heinrich, J., Schneiderbauer, M. \& Menninghaus, W. (2017a) Tears falling on goosebumps: Co-occurrence of emotional lacrimation and emotional piloerection indicates a psychophysiological climax in emotional arousal. Frontiers in Psychology 8:41. Available at: http://doi.org/10.3389/fpsyg. 2017.00041. [arWM]

Wassiliwizky, E., Koelsch, S., Wagner, V., Jacobsen, T. \& Menninghaus, W. (2017b) The emotional power of poetry: Neural circuitry, psychophysiology and compositional principles. Social Cognitive and Affective Neuroscience 12(8):122940. Available at: http://doi.org/10.1093/scan/nsx069. [rWM]

Wassiliwizky, E., Wagner, V., Jacobsen, T. \& Menninghaus, W. (2015) Art-elicited chills indicate states of being moved. Psychology of Aesthetics Creativity and the Arts 9 (4):405-16. Available at: http://doi.org/10.1037/aca0000023. [GG, arWM, JKV]

Weibel, D., Wissmath, B. \& Mast, F. W. (2010) Immersion in mediated environments: The role of personality traits. Cyberpsychology, Behavior, and Social Networking 13(3):251-56. [KF]

Weinstein, L., Xie, X. \& Cleanthous, C. C. (1995) Purpose in life, boredom, and volunteerism in a group of retirees. Psychological Reports 76(2):482. [AE]

Weisberg, D. S. (2015) Pretend play. Wiley Interdisciplinary Reviews: Cognitive Science 6(3):249-61. [NR]

Weisberg, D. S. \& Gopnik, A. (2013) Pretense, counterfactuals, and Bayesian causal models: Why what is not real really matters. Cognitive Science 37(7):1368-81. [NR]

Weisberg, D. S., Sobel, D. M., Goodstein, J. \& Bloom, P. (2013) Young children are reality-prone when thinking about stories. Journal of Cognition and Culture 13(34):383-407. Available at: http://dx.doi.org/10.1163/15685373-12342100. [aWM]

Wezel, J. K. (1785/1971) Versuch über die Kenntniß des Menschen. Athenäum. [aWM]

Whitehouse, H. (1996) Rites of terror: Emotion, metaphor and memory in Melanesian initiation cults. Journal of the Royal Anthropological Institute 2:703-15. [BB]

Whitehouse, H., McQuinn, B., Buhrmester, M. \& Swann, W. B. (2014) Brothers in Arms: Libyan revolutionaries bond like family. Proceedings of the National Academy of Sciences of the United States of America 111(50):17783-85. [BB]

Wildschut, T., Sedikides, C., Arndt, J. \& Routledge, C. (2006) Nostalgia: Content, triggers, functions. Journal of Personality and Social Psychology 91(5):975-93. Available at: http://dx.doi.org/10.1037/0022-3514.91.5.975. [AE, aWM]

Wildschut, T., Sedikides, C., Routledge, C., Arndt, J. \& Cordaro, F. (2010) Nostalgia as a repository of social connectedness: The role of attachment-related avoidance. Journal of Personality and Social Psychology 98(4):573-86. Available at: http://dx.doi.org/10.1037/a0017597. [aWM]

Wilson, T. D. \& Gilbert, D. T. (2003) Affective forecasting. Advances in Experimental Social Psychology 35:345-411. Available at: http://dx.doi.org/10.1016/ S0065-2601(03)01006-2. [aWM]

Wilson, T. D. \& Gilbert, D. T. (2005) Affective forecasting-Knowing what to want Current Directions in Psychological Science 14(3):131-34. Available at: http:// dx.doi.org/10.1111/j.0963-7214.2005.00355.x. [aWM]

Wilson-Mendenhall, C. D., Barrett, L. F. \& Barsalou, L. W. (2013) Neural evidence that human emotions share core affective properties. Psychological Science 24:947-56. [MN]

Winnicott, D. W. (1971) Playing and reality. Basic. [GCC]

Wirth, W., Schramm, H. \& Böcking, S. (2006) Emotionen bei der Rezeption von Unterhaltung. Eine Diskussion klassischer und aktueller Ansätze zur Erklärung medial vermittelter Emotionen. In: Unterhaltung: Konzepte - Formen - Wirkungen, ed. B. Frizzoni \& I. Tomkowiak, pp. 221-46. Chronos. [aWM]

Witasek, S. (1904) Grundzüge der allgemeinen Ästhetik. Barth. [aWM]

Witvliet, C. V. O. \& Vrana, S. R. (2007) Play it again Sam: Repeated exposure to emotionally evocative music polarises liking and smiling responses, and influences other affective reports, facial EMG, and heart rate. Cognition and Emotion 21 (1):3-25. Available at: http://dx.doi.org/10.1080/02699930601000672. [aWM]

Wohl, H. (2015) Community sense: The cohesive power of aesthetic judment. Sociological Theory 33(4):299-326. [EB]

Xygalatas, D., Mitkidis, P., Fischer, R., Reddish, P., Skewes, J., Geertz, A. W., Roepstorff, A. \& Bulbulia, J. (2013) Extreme rituals promote prosociality. Psychological Science 24:1602-05. [BB]

Yanal, R. J. (1996) The paradox of suspense. British Journal of Aesthetics 36(2):14658. Available at: http://dx.doi.org/10.1093/bjaesthetics/36.2.146. [aWM]

Zajonc, R. B. (1968) Attitudinal effects of mere exposure. Journal of Personality and Social Psychology 9(2, Pt.2):1-27. Available at: http://dx.doi.org/10.1037/ h0025848. [aWM, MN]

Zangwill, N. (2014) Aesthetic judgment. In: The Stanford Encyclopedia of philosophy, Fall 2014 edition, ed. E. N. Zalta. Metaphysics Research Lab, Stanford University. Available at: https://plato.stanford.edu/archives/fall2014/entries/ aesthetic-judgment/. [EB]

Zelle, C. (1987) Angenehmes Grauen. Literaturhistorische Beiträge zur Ästhetik des Schrecklichen im achtzehnten Jahrhundert. Meiner. [aWM]

Zentner, M., Grandjean, D. \& Scherer, K. R. (2008) Emotions evoked by the sound of music: Characterization, classification, and measurement. Emotion 8(4):494521. Available at: http://dx.doi.org/10.1037/1528-3542.8.4.494. [aWM]

Zickfeld, J. H., Schubert, T. W., Seibt, B. \& Fiske, A. P. (2017) Empathic concern is part of a more general communal emotion. Frontiers in Psychology 8:723. Available at: http://doi.org/10.3389/fpsyg.2017.00723. [TWS, JKV] 
References/Menninghaus et al.: The Distancing-Embracing model of the enjoyment of negative emotions in art reception

Zillmann, D. (1971) Excitation transfer in communication-mediated aggressive behavior. Journal of Experimental Social Psychology 7(4):419-34. Available at: http://dx.doi.org/10.1016/0022-1031(71)90075-8. [aWM]

Zillmann, D. (1980) Anatomy of suspense. In: The entertainment functions of television, ed. P. H. Tannenbaum, pp. 133-63. Erlbaum. [aWM]

Zillmann, D. (1988) Mood management: Using entertainment to full advantage. In: Communication, social cognition, and affect, vol. 31, ed. L. Donohew, H. E. Sypher \& E. T. Higgins, pp. 147-71. Erlbaum. [aWM]

Zillmann, D. (1991) Empathy: Affect from bearing witness to the emotions of others. In: Responding to the screen, ed. J. Bryant \& D. Zillmann, pp. 135-67. Erlbaum. [aWM]

Zillmann, D. (2006) Dramaturgy for emotions from fictional narration. In: Psychology of entertainment, ed. J. Bryant \& P. Vorderer, pp. 215-38. Erlbaum. [aWM]

Zillmann, D. \& Weaver, J. B. (1996) Gender-socialization theory of reactions to horror. In: Horror films. Current research on audience preferences and reactions, ed. J. B. Weaver \& R. Tamborini, pp. 81-101.

Erlbaum. [aWM]

Zillmann, D., Weaver, J. B., Mundorf, N. \& Aust, C. F. (1986) Effects of an oppositegender companion's affect to horror on distress, delight, and attraction. Journal of Personality and Social Psychology 51(3):586-94. Available at: http://dx.doi. org/10.1037/0022-3514.51.3.586. [aWM]

Zuckerman, M. (1979) Sensation seeking: Beyond the optimal level of arousal. Erlbaum. [aWM]

Zwaan, R. A. (1994) Effect of genre expectations on text comprehension. Journal of Experimental Psychology: Learning Memory and Cognition 20(4):920-33. Available at: http://dx.doi.org/10.1037/0278-7393.20.4.920. $[\mathrm{aWM}]$

Zwaan, R. A. (1999) Situation models: The mental leap into imagined worlds. Current Directions in Psychological Science 8(1):15-18. Available at: http://dx. doi.org/10.1111/1467-8721.00004. [aWM] 